\title{
Landtechnische Methoden zur Erfassung von Bodenverdichtungen
}

\author{
Dissertation \\ zur Erlangung des Doktorgrades \\ der Fakultät für Agrarwissenschaften \\ der Georg-August-Universität Göttingen
}

vorgelegt von

Stephan Denker

geboren am 28.09.1974 in Lübeck

Göttingen, Mai 2011 
D 7

Referenten: 1. Prof. Dr. Wolfgang Lücke

2. Prof. Dr. Bernward Märländer

3. Prof. Dr. Elke Pawelzik

eingereicht:

20. Mai 2011

Tag der mündlichen Prüfung: 21. Juli 2011 
Inhaltsverzeichnis

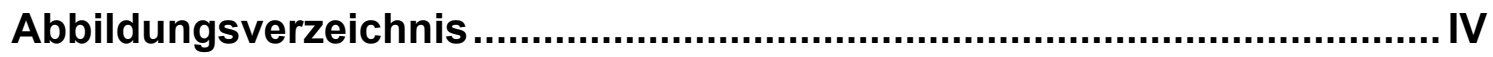

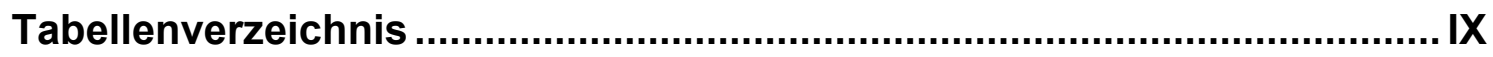

Verzeichnis der verwendeten Formeln.....................................................

Verzeichnis der Tabellen im Anhang .......................................................... XI

Abkürzungsverzeichnis ....................................................................

Verzeichnis der verwendeten Symbole ..................................................XVIII

1. Einleitung und Zielsetzung der Arbeit................................................. 1

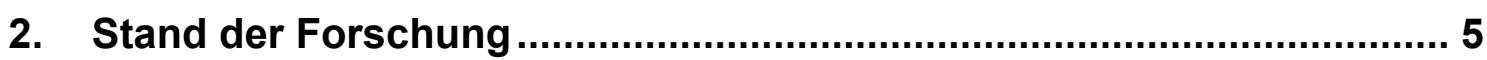

3. Material und Methoden .................................................................. 18

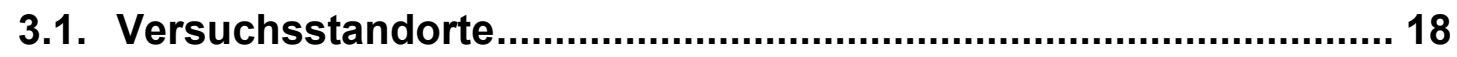

3.1.1. Soester Börde: Versuchsgut Merklingsen ................................... 19

3.1.2. Östliches Hügelland: Versuchsgüter Hohenschulen und Lindhof... 21

3.2. Versuchsaufbau und -durchführung .............................................. 23

3.2.1. Faktoren und Faktorstufen ..................................................... 23

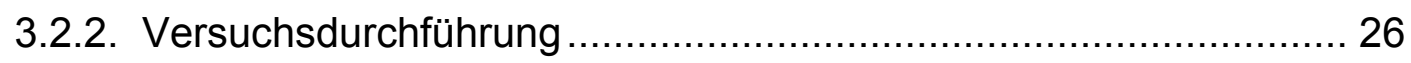

3.3. Messsysteme und Messwerterfassung ........................................... 28

3.3.1. Movis: Mechanischer Lasteintrag unter definierten Bedingungen.. 28

3.3.2. Erfassung des vertikalen Eindringwiderstandes........................... 30

3.3.3. TASIS: Erfassung des horizontalen Eindringwiderstandes .............. 31

3.3.4. Bodenphysikalische Untersuchungen ........................................ 36

3.3.5. Erfassung weiterer Parameter ................................................. 37

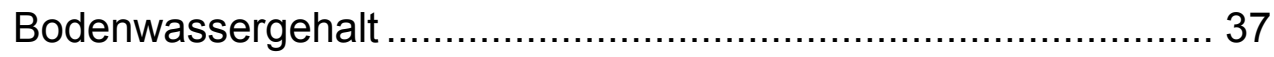

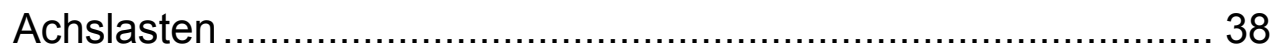

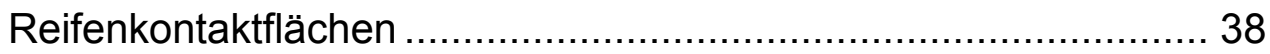

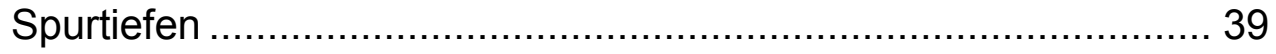


3.4. Datenverarbeitung und statistische Auswertung

4. Ergebnisse

4.1. Äußere Bedingungen der Versuchsdurchführung 42

4.2. Kontaktflächen und Kontaktflächendrücke

4.3. Spurtiefen 47

4.4. Vertikaler Eindringwiderstand 51

4.4.1. Ausprägung des vertikalen Eindringwiderstandes auf den einzelnen Versuchsflächen vor Versuchsbeginn. 52

4.4.2. Effekte der Faktoren Standort, Bodenbearbeitung sowie besonderer Wetterereignisse

4.4.3. Entwicklung des vertikalen Eindringwiderstandes über den Versuchszeitraum 60

4.4.4. Effekte einer mechanischen Belastung 63

4.4.5. Einflüsse der Fahrwerksparameter Radlast und Reifeninnendruck 67

4.5. Horizontaler Eindringwiderstand. 71

4.5.1. Datensatzstruktur und Effekte der Hauptfaktoren 71

4.5.2. Horizontale Eindringwiderstände nach Flächen 75

Schleswig-Holstein, konservierende Bodenbewirtschaftung 75

Schleswig-Holstein, konventionelle Bodenbewirtschaftung ..... 77

Nordrhein-Westfalen, konservierende Bodenbewirtschaftung....... 79

Nordrhein-Westfalen, konventionelle Bodenbewirtschaftung. 81

4.5.3. Effekte der Hauptfaktoren Radlast und Reifeninnendruck auf den horizontalen Eindringwiderstand 84

4.5.4. Der Sonderbereich Geophysik 86

4.5.5. Untersuchungen zur Eingriffsintensität von TASIS in das Bodengefüge 91 
4.6. Betrachtung der Zusammenhänge von vertikalen und horizontalen Eindringwiderständen 93

4.6.1. Betrachtung der Korrelationen zwischen vertikalen und horizontalen Eindringwiderständen 93

4.6.2. Der Ruhedruckkoeffizient als rechnerische Größe aus horizontalem und vertikalem Eindringwiderstand 95

4.7. Bodenkundliche Untersuchungen 99

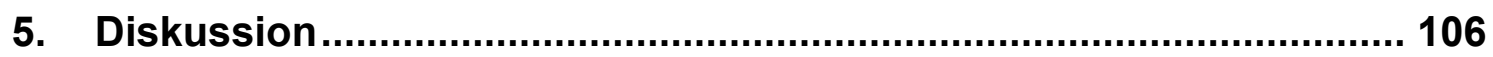

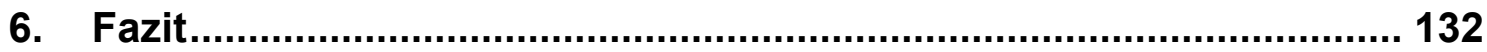

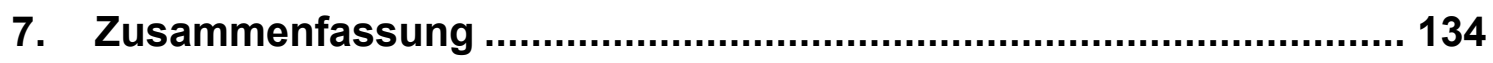

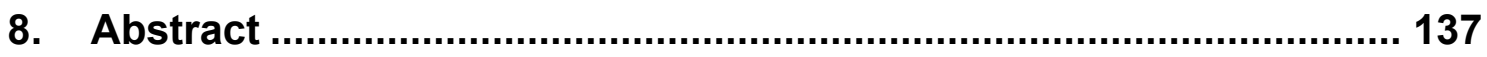

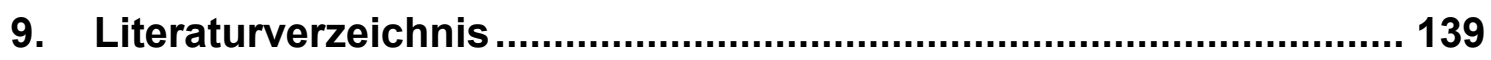

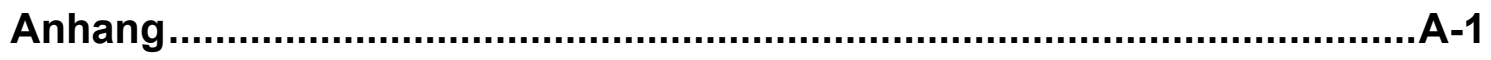




\section{Abbildungsverzeichnis}

Abbildung 1: Geografische Lage der Versuchsgüter Merklingsen (NRW),

Hohenschulen und Lindhof (jeweils SH)

(Quelle: Google Earth).

Abbildung 2: Versuchsgut Merklingsen (Mitte) mit den eingezeichneten

Versuchsflächen $\mathrm{NRW}_{\text {kons }}$ (links) und $\mathrm{NRW}_{\text {konv }}$ (rechts)

(Quelle: Google Earth, Aufnahmedatum 31. Oktober 2004)..... 20

Abbildung 3: Veruchsgut Hohenschulen (links) mit den Teilflächen der

Versuchsflächen $\mathrm{SH}_{\text {kons }}$ (Quelle: Google Earth,

Aufnahmedatum 01. Januar 2000).

Abbildung 4: Versuchsgut Lindhof mit der angrenzenden Versuchsfläche

$\mathrm{SH}_{\text {konv }}$ (Quelle: Google Earth, Aufnahmedatum

01. Januar 2000).

Abbildung 5: Versuchsdesign am Beispiel der Fläche NRW konv $_{\text {. }}$

Abbildung 6: Movis (Motion Vehicle Information System) in diagonaler

Draufsicht und Seitenansicht.

Abbildung 7: CAD-Zeichnung eines einzelnen Schares und der gesamten

Schargruppe des Messsystems TASIS

Abbildung 8: Scharkörper von TASIS mit seinen Funktionselementen

in der Seitenansicht.

Abbildung 9: Einzelkomponenten des Messsystems TASIS.

Abbildung 10: Darstellung des Beprobungsschemas innerhalb einer

Versuchsparzelle.

Abbildung 11: Gravimetrische Bodenwassergehalte [\%] der

Versuchsflächen zu den Untersuchungsterminen in verschiedenen Bodentiefen ( $n=2074, \mathrm{GD}_{5 \%} \approx 4,3 \%$,

$\bar{x}$ über 2 Zeitpunkte, 4 Messgruben, $5 \mathrm{Wdh}$.). 
Abbildung 12: Spurtiefen der Befahrungen nach Fläche und Belastungsvariante $\left(\mathrm{n}=1.980, \mathrm{GD}_{5 \%} \approx 0,42 \mathrm{~cm}\right.$, $\bar{x}$ über 5 Termine, 2 Blöcke, 15 Wdh.). 48

Abbildung 13: Mittelwerte der gemessenen Spurtiefen ( \pm Standardfehler) bei verschie-denen Radlasten in Abhängigkeit des angewendeten Bodenbearbeitungssystems ( $\mathrm{n}=1.980$, $\mathrm{GD}_{5 \%} \approx 0,21 \mathrm{~cm}, \overline{\mathrm{x}}$ über 2 Zeitpunkte, 2 Reifeninnendrücke, 2 Blöcke, 15 Wdh.).

Abbildung 14: Mittelwerte der gemessenen Spurtiefen ( \pm Standardfehler) bei verschiedenen Radlasten in Abhängigkeit des eingestellten Reifeninnendruckes $(n=1.980$, $\mathrm{GD}_{5 \%} \approx 0,21 \mathrm{~cm}, \overline{\mathrm{x}}$ über 2 Zeitpunkte, 2 Reifeninnendrücke, 2 Blöcke, 15 Wdh.). 50

Abbildung 15: Vertikale Eindringwiderstände der Versuchsflächen vor Versuchsbeginn 2006 in den Darstellungsweisen Boxplot(links) und Liniendiagramm (rechts) $\left(\mathrm{GD}_{5 \%} \approx 0,1 \mathrm{MPa}\right)$.

Abbildung 16: Effekte einer Befahrung auf den vertikalen

Eindringwiderstand nach zuvor erfolgter wendender

Bodenbearbeitung in den Messtiefenbereichen $5-14 \mathrm{~cm}$ und $18-27 \mathrm{~cm}\left(\mathrm{n}=780, \mathrm{GD}_{5 \%} \approx 0,30 \mathrm{MPa}, \bar{x}\right.$ über 2 Blöcke, 6 Wdh.) 55

Abbildung 17: Effekte einer Befahrung auf den vertikalen

Eindringwiderstand nach zuvor erfolgter wendender Bodenbearbeitung im Messtiefenbereich $30-39 \mathrm{~cm}$ ( $\mathrm{n}=390, \mathrm{GD}_{5 \%} \approx 0,60 \mathrm{MPa}, \overline{\mathrm{x}}$ über 2 Blöcke, $6 \mathrm{Wdh}$.).

Abbildung 18: Effekte eines Niederschlagsereignisses auf die vertikalen

Eindringwiderstände vor und nach einer Befahrung am Standort Schleswig-Holstein im Herbst 2006 in den Messtiefenbereichen $5-14 \mathrm{~cm}$ und $18-27 \mathrm{~cm}(\mathrm{n}=704$, $\mathrm{GD}_{5 \%} \approx 0,42 \mathrm{MPa}, \overline{\mathrm{x}}$ über 2 Blöcke, $6 \mathrm{Wdh}$.). 58 
Abbildung 19: Effekte eines Niederschlagsereignisses auf die vertikalen Eindringwiderstände vor und nach einer Befahrung am Standort Schleswig-Holstein im Herbst 2006 in den Messtiefenbereichen $30-39 \mathrm{~cm}$ und $50-59 \mathrm{~cm}$ ( $n=703$, $\mathrm{GD}_{5 \%} \approx 0,73 \mathrm{MPa}, \overline{\mathrm{x}}$ über 2 Blöcke, $6 \mathrm{Wdh}$.). 59

Abbildung 20: Vertikale Eindringwiderstände nach Fläche, Termin und Messtiefenbereich $\left(\mathrm{n}=15.362, \mathrm{GD}_{5 \%} \approx 0,12 \mathrm{MPa}, \overline{\mathrm{x}}\right.$ über 2 Zeitpunkte, 6 Varianten, 2 Blöcke, 6 Wdh.).

Abbildung 21: Vertikale Eindringwiderstände [MPa] nach erfolgter

Befahrung mit unterschiedlichen Radlasten bei konstantem

Reifeninnendruck (160 kPa) auf $\operatorname{NRW}_{\text {konv }}(\mathrm{n}=180, \bar{x}$ über 5

Termine, 3 Blöcke, 6 Wdh.). 68

Abbildung 22: Vertikale Eindringwiderstände [MPa] bei konstanten

Radlasten und unterschiedlichen Reifeninnendrücken am

Standort Schleswig-Holstein in den Messtiefenbereichen

$30-39 \mathrm{~cm}$ und $50-59 \mathrm{~cm}\left(\mathrm{n}=186, \mathrm{GD}_{5 \%} \approx 0,37 \mathrm{MPa}\right.$,

$\bar{x}$ über 2 Blöcke, 6 Wdh.).

Abbildung 23: Horizontale Eindringwiderstände [MPa] nach Flächen und Messtiefen $\left(\mathrm{n}=3.855, \mathrm{GD}_{5 \%} \approx 0,03 \mathrm{MPa}, \overline{\mathrm{x}}\right.$ über 5 Termine, 6 Varianten, 2 Blöcke, 6 Wdh.). 73

Abbildung 24: Vertikale Eindringwiderstände [MPa] nach erfolgter

Befahrung nach Flächen und Messtiefen ( $n=5.710$, $\mathrm{GD}_{5 \%} \approx 0,12 \mathrm{MPa}, \overline{\mathrm{x}}$ über 5 Termine, 6 Varianten, 2 Blöcke, 6 Wdh.).

Abbildung 25: Horizontale Eindringwiderstände [MPa] der Fläche $\mathrm{SH}_{\mathrm{kons}}$ im Jahr 2008 in den Messtiefenbereichen $18-27 \mathrm{~cm}$ und 30 - $39 \mathrm{~cm}$ nach Belastungsvariante $(\mathrm{n}=87$, $\mathrm{GD}_{5 \%} \approx 0,09 \mathrm{MPa}, \overline{\mathrm{x}}$ über 2 Blöcke, $6 \mathrm{Wdh}$.). 76

Abbildung 26: Horizontale Eindringwiderstände [MPa] gemessen auf $\mathrm{SH}_{\text {konv }}$ im Herbst 2006 im Messtiefenbereich $18-27 \mathrm{~cm}$ ( $\mathrm{n}=95, \mathrm{GD}_{5 \%} \approx 0,12 \mathrm{MPa}, \overline{\mathrm{x}}$ über 2 Blöcke, $6 \mathrm{Wdh}$.). 78 
Abbildung 27: Horizontale Eindringwiderstände [MPa] gemessen auf $\mathrm{SH}_{\text {konv }}$ im Frühjahr 2008 in den Messtiefenbereichen 5 $14 \mathrm{~cm}$ und $18-27 \mathrm{~cm}\left(\mathrm{n}=192, \mathrm{GD}_{5 \%} \approx 0,09 \mathrm{MPa}, \overline{\mathrm{x}}\right.$ über 2 Blöcke, 6 Wdh.). 79

Abbildung 28: Horizontale Eindringwiderstände [MPa] der Fläche NRW konv in den Messtiefenbereichen $5-14 \mathrm{~cm}$ und $18-29 \mathrm{~cm}$ nach Jahr und Belastungsvariante $\left(\mathrm{n}=864, \mathrm{GD}_{5 \%} \approx 0,04 \mathrm{MPa}\right.$, x̄ über 3 Blöcke, 6 Wdh.).

Abbildung 29: Horizontale Eindringwiderstände [MPa] der Fläche NRW konv im Messtiefenbereichen 30 - $39 \mathrm{~cm}$ nach Jahr und Belastungsvariante $\left(\mathrm{n}=432, \mathrm{GD}_{5 \%} \approx 0,12 \mathrm{MPa}, \overline{\mathrm{x}}\right.$ über 3 Blöcke, 6 Wdh.). 83

Abbildung 30: Effekte einer Erhöhung des Reifeninnendruckes bei 7,5 Mg Radlast auf den horizontalen Eindringwiderstand [MPa] bei konservierender und konventioneller Bodenbewirtschaftung am Standort Schleswig-Holstein $(n=510$, $\mathrm{GD}_{5 \%} \approx 0,12 \mathrm{MPa}, \overline{\mathrm{x}}$ über 3 Termine, 2 Blöcke, 6 Wdh.). 86

Abbildung 31: Trenddiagramm der horizontalen Eindringwiderstände [MPa] nach Versuchsfläche, Messtiefenbereich und Radlast unter Berücksichtigung des Sonderbereiches GEO (nur Daten aus 2008).

Abbildung 32: Horizontale Eindringwiderstände [MPa] nach Messtiefenbereich und Radlast für konservierende und konventionelle Bodenbearbeitung (Frühjahr 2008, ohne nur Schlepper). 88

Abbildung 33: Entwicklung der horizontalen Eindringwiderstände [MPa] auf konventionell bewirtschafteten Flächen bei steigender mechanischer Belastung in den Messtiefenbereichen $5-14 \mathrm{~cm}$ und $18-27 \mathrm{~cm}$ 89 
Abbildung 34: Entwicklung der horizontalen Eindringwiderstände [MPa] auf konventionell bewirtschafteten Flächen bei steigender mechanischer Belastung in den Messtiefenbereichen $30-39 \mathrm{~cm}$. 90

Abbildung 35: Horizontale Eindringwiderstände [MPa] in 2008 nach Bodenbewirtschaftung und Messtiefenbereich in Abhängigkeit des TASIS-Einsatzes an selber Stelle ( $\mathrm{n}=3.948, \mathrm{GD}_{5 \%} \approx 0,03 \mathrm{MPa}, \overline{\mathrm{x}}$ über 2 Standorte, 6 Varianten, 2 Blöcke, 6 Wdh.).

Abbildung 36: Punktwolken-Diagramme mit Pearson-Korrelationskoeffizienten $(* * \triangleq$ signifikant mit $p \leq 0,01)$ nach Standort und Bodenbearbeitungssystem ( $r_{\text {gesamt }}=0,60^{* *}, n=3.773, \bar{x}$ über 3 Termine, 3 Messtiefen, 6 Varianten, 2 Blöcke, 6 Wdh.) 94

Abbildung 37: Ruhedruckkoeffizienten und horizontale und vertikale Eindringwiderstände nach Bodenbearbeitung und Messtiefenbereich $\left(\mathrm{GD}_{5 \%} \approx 0,02, \overline{\mathrm{x}}\right.$ über 2 Standorte, 3 Termine, 6 Varianten, 2 Blöcke, 6 Wdh.). 96

Abbildung 38: Ruhedruckkoeffizienten und horizontale und vertikale Eindringwiderstände nach Versuchsfläche und Radlast in den Messtiefen $5-14 \mathrm{~cm}$ und $18-27 \mathrm{~cm}\left(G D_{5 \%} \approx 0,05, \bar{x}\right.$ über 3 Termine, 2 Reifeninnendrücke je Radlast, 12 Wdh.)..... 98

Abbildung 39: Luftkapazität (\%) und gesättigte Wasserleitfähigkeit (cm/d) von $\mathrm{NRW}_{\text {konv }}$ in Abhängigkeit von Belastungsvariante und Messtiefenbereich nach Versuchsende. Die gestrichelten Linien kennzeichnen die Schadschwellen nach LEBERT ET AL. $(2004)(n=20)$. 102 


\section{Tabellenverzeichnis}

Tabelle 1: Faktorstufenkombination für den Reifen Michelin MegaXBib ${ }^{\circledR}$ 650/75 R32

Tabelle 2: Belastungsvarianten der Befahrungen ab Herbst 2006

Tabelle 3: Faktorstufenkombinationen für Michelin MachXBib 650/75 R38

(nur NRW Frühjahr 2006) 26

Tabelle 4: Mittlere Kontaktflächen [ $\left.\mathrm{cm}^{2}\right]$ der Belastungsvarianten nach

Fläche und Termin für Michelin MegaXBib ${ }^{\circledR}$ 650/75 R32

(MachXBib 650/75 R38 für Frühjahr 2006, x über 6 Varianten) 46

Tabelle 5: Vertikale Eindringwiderstände in Abhängigkeit des Zeitpunktes der Beprobung ( $n=15.362, \bar{x}$ über 4 Messtiefenbereiche (MTB), 6 Varianten, 2 Blöcke, 6 Wdh.).

Tabelle 6: Horizontale Eindringwiderstände [MPa] nach Fläche und Bodenbearbeitung ( $\bar{x}$ über 3 Termine, 3 Messtiefen,

6 Varianten, 2 Blöcke, 6 Wdh.).

Tabelle 7: Pearson-Korrelationskoeffizienten $\left({ }^{* *} \triangleq\right.$ signifikant mit $\left.p \leq 0,01\right)$ zwischen horizontalem und vertikalem Eindringwiderstand nach Versuchsfläche und Messtiefenbereich ( $\bar{x}$ über 3 Termine, 6 Varianten, 2 Blöcke, 6 Wdh.). 95

Tabelle 8: Ergebnisse der Stechzylinderproben der Versuchfläche $\mathrm{NRW}_{\text {konv }}$ (Mittelwerte, $\mathrm{n}=20$ ). 100

Tabelle 9: Ergebnisse der Spatendiagnose vom 06.10.2008 auf der Fläche NRW $_{\text {konv }}$ (nach DBG 2005). 


\section{Verzeichnis der verwendeten Formeln}

Formel 1: Berechnung des Gesamtporenvolumens ............................. 37

Formel 2: Berechnung des gravimetrischen Wassergehaltes ...................... 38

Formel 3: Berechnung der Reifenkontaktfläche .................................. 39

Formel 4: Beziehung zwischen Hauptspannungen und

Ruhedruckkoeffizienten.................................................... 40

Formel 5: Formel zur direkten Berechnung des

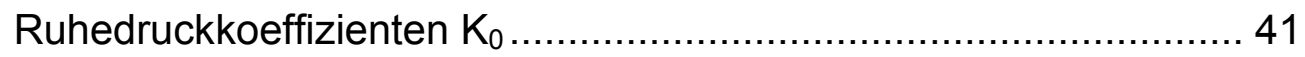




\section{Verzeichnis der Tabellen im Anhang}

Tabelle A 1: Temperatur und Niederschlag auf den Versuchsflächen während der Projektlaufzeit..

Tabelle A 2: Bodeneigenschaften der Versuchsfläche $\mathrm{SH}_{\mathrm{kons}}$ (nach ZINK 2009)

Tabelle A 3: Bodeneigenschaften der Versuchsfläche $\mathrm{SH}_{\mathrm{konv}}$

(nach ZINK 2009)

Tabelle A 4: Bodeneigenschaften der Versuchsfläche $N_{R} W_{\text {kons }}$

(nach ZINK 2009)

Tabelle A 5: Bodeneigenschaften der Versuchsfläche $\mathrm{NRW}_{\text {konv }}$ (nach ZINK 2009)

Tabelle A 6: Bodenbearbeitung und Fruchtfolge der Versuchsflächen am Standort Nordrhein-Westfalen 2000 - 2008.

Tabelle A 7: Bodenbearbeitung und Fruchtfolge der Versuchsflächen am Standort Schleswig-Holstein 2000 - 2008

Tabelle A 8: Kennzahlen des verwendeten Reifens Michelin MegaXBib (Michelin 2006)

Tabelle A 9: Gravimetrischer Wassergehalt [\%] nach Fläche, Messtiefe und Untersuchungstermin. A-6

Tabelle A 10: Mittlere Kontaktflächendrücke [kPa] der Belastungsvarianten nach Termin und Fläche

Tabelle A 11: Mittelwerte der gemessenen Spurtiefen (ohne Variante nur Schlepper) nach Fläche, Radlast (RL) und Reifeninnendruck (RID) gemittelt über den Versuchszeitraum

Tabelle A 12: Mittelwerte der gemessenen Spurtiefen bei verschiedenen Radlasten $(R L)$ in Abhängigkeit der angewendeten Bodenbearbeitung (BB) 
Tabelle A 13: Mittelwerte der gemessenen Spurtiefen bei verschiedenen Radlasten ( $R L)$ in Abhängigkeit des eingestellten

Reifeninnendruckes (RID).

Tabelle A 14: Mittelwerte der vertikalen Eindringwiderstände nach Fläche und Messtiefenbereich (MTB) vor Versuchsbeginn

Tabelle A 15: Mittelwerte der vertikalen Eindringwiderstände nach Fläche und Variante vor Versuchsbeginn

Tabelle A 16: Effekte einer Befahrung auf den vertikalen Eindringwiderstand nach zuvor erfolgter Bodenbearbeitung

Tabelle A 17: Effekte eines Niederschlagsereignisses auf die vertikalen Eindringwiderstände vor und nach einer Bef. am Standort SH im Herbst 2006

Tabelle A 18: Gravimetrische Bodenwassergehalte [\%] der Flächen $\mathrm{SH}_{\text {kons }}$ und $\mathrm{SH}_{\text {konv }}$ im Herbst 2006 zum Zeitpunkt der Vertikalpenetrometermessungen vor der Bef. (22./23.10.2006) und nach der Bef. (30./31.10.2006)

Tabelle A 19: Vertikale Eindringwiderstände nach Fläche, kalendarischem Termin und Messtiefenbereich

Tabelle A 20: Vertikaler Eindringwiderstand [MPa] bei Varianten unterschiedlicher Radlasten bei 160kPa Reifeninnendruck nach Fläche und Messtiefenbereich

Tabelle A 21: Vertikaler Eindringwiderstand [MPa] bei Varianten unterschiedlicher Radlasten bei 250 kPa Reifeninnendruck nach Fläche und Messtiefenbereich

Tabelle A 22: Vertikaler Eindringwiderstand [MPa] bei Varianten mit unterschiedlichem Reifeninnendruck (RID) bei 3,3 Mg Radlast nach Fläche und Messtiefenbereich (MTB).

Tabelle A 23: Vertikaler Eindringwiderstand [MPa] bei Varianten mit unterschiedlichem Reifeninnendruck (RID) bei 6,3 Mg Radlast nach Fläche und Messtiefenbereich (MTB). 
Tabelle A 24: Vertikaler Eindringwiderstand [MPa] bei Varianten mit unterschiedlichem Reifeninnendruck bei 7,5 Mg Radlast nach Fläche und Messtiefenbereich (ohne Block 3)

Tabelle A 25: Horizontale Eindringwiderstände [MPa] nach Jahr und Messtiefenbereich gemittelt über Varianten und Flächen

Tabelle A 26: Horizontale Eindringwiderstände [MPa] der Fläche $\mathrm{SH}_{\text {kons }}$ im Jahr 2006 nach Messtiefenbereich und Belastungsvariante

Tabelle A 27: Horizontale Eindringwiderstände [MPa] der Fläche $\mathrm{SH}_{\text {kons }}$ im Jahr 2007 nach Messtiefenbereich und Belastungsvariante

Tabelle A 28: Horizontale Eindringwiderstände [MPa] der Fläche $\mathrm{SH}_{\text {kons }}$ im Jahr 2008 nach Messtiefenbereich und Belastungsvariante

Tabelle A 29: Horizontale Eindringwiderstände [MPa] der Fläche $\mathrm{SH}_{\text {konv }}$ im Jahr 2006 nach Messtiefenbereich und Belastungsvariante

Tabelle A 30: Horizontale Eindringwiderstände [MPa] der Fläche $\mathrm{SH}_{\text {konv }}$ im Jahr 2007 nach Messtiefenbereich und Belastungsvariante

Tabelle A 31: Horizontale Eindringwiderstände [MPa] der Fläche $\mathrm{SH}_{\mathrm{konv}}$ im Jahr 2008 nach Messtiefenbereich und Belastungsvariante

Tabelle A 32: Horizontale Eindringwiderstände [MPa] der Fläche $\mathrm{NRW}_{\text {kons }}$ im Jahr 2006 nach Messtiefenbereich und Belastungsvariante .... A-36

Tabelle A 33: Horizontale Eindringwiderstände [MPa] der Fläche $\mathrm{NRW}_{\text {kons }}$ im Jahr 2007 nach Messtiefenbereich und Belastungsvariante .... A-37

Tabelle A 34: Horizontale Eindringwiderstände [MPa] der Fläche $\mathrm{NRW}_{\text {kons }}$ im Jahr 2008 nach Messtiefenbereich und Belastungsvariante .... A-38

Tabelle A 35: Horizontale Eindringwiderstände [MPa] der Fläche $\mathrm{NRW}_{\text {konv }}$ im Jahr 2006 nach Messtiefenbereich und Belastungsvariante .... A-39

Tabelle A 36: Horizontale Eindringwiderstände [MPa] der Fläche NRW $_{\text {konv }}$ im Jahr 2007 nach Messtiefenbereich und Belastungsvariante .... A-40

Tabelle A 37: Horizontale Eindringwiderstände [MPa] der Fläche $\mathrm{NRW}_{\text {konv }}$ im Jahr 2008 nach Messtiefenbereich und Belastungsvariante .... A-41 
Tabelle A 38: Horizontale Eindringwiderstände 2008 nach Fläche, Messtiefe und Radlast unter Berücksichtigung des

Sonderbereiches Geo

Tabelle A 39: Horizontale Eindringwiderstände 2008 nach Bodenbearbeitung (BB), Messtiefenbereich (MTB) und Radlast (RL) unter Berücksichtigung des Sonderbereiches Geo A-44

Tabelle A 40: Effekt des Faktors Radlast bei 160 kPa Reifeninnendruck auf die horizontalen Eindringwiderstände [MPa] nach Jahr, Fläche und Messtiefenbereich

Tabelle A 41: Effekt des Faktors Radlast bei 250 kPa Reifeninnendruck auf die horizontalen Eindringwiderstände [MPa] nach Jahr, Fläche und Messtiefenbereich

Tabelle A 42: Effekt des Faktors Reifeninnendruck bei 3,3 Mg Radlast auf die horizontalen Eindringwiderstände [MPa] nach Jahr, Fläche und Messtiefenbereich

Tabelle A 43: Effekt des Faktors Reifeninnendruck bei 6,3 Mg Radlast auf die horizontalen Eindringwiderstände [MPa] nach Jahr, Fläche und Messtiefenbereich A-51

Tabelle A 44: Effekt des Faktors Reifeninnendruck bei 7,5 Mg Radlast auf die horizontalen Eindringwiderstände [MPa] nach Jahr, Fläche und Messtiefenbereich

Tabelle A 45: Horizontaler Eindringwiderstand [MPa] nach Jahr und Fläche in Abhängigkeit der Anzahl der zuvor erfolgten TASISEinsätze an selben Stelle.

Tabelle A 46: Horizontaler Eindringwiderstand [MPa] nach Bodenbearbeitung (BB) und Messtiefenbereich in Abhängigkeit der Anzahl der zuvor erfolgten TASIS-Einsätze an selber Stelle A-56

Tabelle A 47: Ruhedruckkoeffizient nach Fläche, Messtiefenbereich und Radlast 
Tabelle A 48: Ruhedruckkoeffizient nach Bodenbearbeitung (BB) und Messtiefenbereich (MTB) ........................................ A-58

Tabelle A 49: Ergebnisse der erweiterten Spatendiagnose vom 06.10.2008 auf der Fläche NRW (nach DBG 2005) A-59 


\section{Abkürzungsverzeichnis}

\section{Abkürzung oder Symbol}

BB

BBodSchG

Bef.

bzw.

CAU

d

$d / w-$ Relation

DBG

DMS

EW

Fa.

$\mathrm{FH}-\mathrm{SWF}$

FK

GD

GEO

Gew.

GFP

GIS

GPS

grav.

gWG

hEW

$\mathrm{Hz}$

i. A.

i. d. R.

k. A.

kf

$\mathrm{kPa}$

Kr.-egge

LJM

LSD

m

$\mathrm{M}$

$\mathrm{Mg}$
Bedeutung

Bodenbearbeitung

Bundes-Bodenschutzgesetz

Befahrung

beziehungsweise

Christian-Albrechts-Universität zu Kiel

Tag

Relation Arbeitstiefe / Scharbreite nach GoDWIN 2007

Deutsche Bodenkundliche Gesellschaft

Dehnmessstreifen

Eindringwiderstand [MPa]

Firma

Fachhochschule Südwestfalen, Agrarwirtschaft Soest

Feldkapazität

Grenzdifferenz

Sonderbereich Geophysik

Gewicht

Gute fachliche Praxis

Geografisches Informationssystem

Global Positioning System

gravimetrisch

gravimetrischer Wassergehalt [\%]

horizontaler Eindringwiderstand [MPa]

Hertz

im Allgemeinen

in der Regel

keine Angabe

gesättigte Wasserleitfähigkeit [cm/d]

Kilopascal (100 kPa = 1 bar)

Kreiselegge

Langjähriges Mittel

geringste signifikante Differenz

(Least Significant Difference)

Meter

arithmetischer Mittelwert

Megagramm (1 Mg = 1 to $)$ 
Movis

$\mathrm{MPa}$

MTB

n

n. E.

Nds.

NRW

$\mathrm{p}$

PC

PCMCIA

$P d$

RDK

RID

$\mathrm{RL}$

RTK

$\mathrm{S}$

Sch.-egge

Sch.-grubber

SD

SED

$\mathrm{SH}$

SHA

sog.

SPR-egge

SR

SW

TASIS

Temp.

u. U.

Univ.-egge

üNN

vEW
Motion Vehicle Information System

(vormalige Bezeichnung: Horizontalmesswagen, System der FH-SWF zum definierten

Lasteintrag auf landwirtschaftlichen Flächen)

Megapascal

Messtiefenbereich

(5 - $14 \mathrm{~cm}, 18-27 \mathrm{~cm}, 30-39 \mathrm{~cm}$ )

Anzahl

nach Einschätzung

Niederschlag

Nordrhein-Westfalen

Irrtumswahrscheinlichkeit

Personal Computer

Personal Computer Memory Card International

Association

Packungsdichte des Bodens

Ruhedruckkoeffizient $\mathrm{K}_{0}$ [-]

(horizontale Hauptspannung $\delta_{1} /$ vertikale

Hauptspannung $\delta_{2}$ )

Reifeninnendruck [kPa]

Radlast (3,3 Mg, 6,3 Mg, 7,5 Mg)

Real Time Kinematic

(GPS-Systeme mit $\pm 2 \mathrm{~cm}$ Genauigkeit)

Sekunde

Scheibenegge

Scheibengrubber

Standardabweichung

Fehler des Mittelwerts

(Standar Error Deviation)

Schleswig-Holstein

Sommerhafer

sogenannt

Spatenrollegge

Sommerroggen

Sommerweizen

Tactile Soil Information System

(Horizontalpenetrometer der FH-SWF)

Temperatur

unter Umständen

Universalegge

über Normalnull (Meeresspiegel)

vertikaler Eindringwiderstand [MPa] 
Vol.

VP

VPN

vs.

Wdh.

$W_{\mathrm{g}}$

WG

WH

WR

WW

z. T.

$\mathrm{ZR}$

Zykl.
Volumen

Vertikalpenetrometer

Virtual Private Network

versus

Wiederholung

gravimetrischer Wassergehalt [Gew.-\%]

Wintergerste

Winterhafer

Winterraps

Winterweizen

zum Teil

Zuckerrübe

zyklische Radbelastung 


\section{Verzeichnis der verwendeten Symbole}

\begin{tabular}{|c|c|}
\hline Abkürzung oder Symbol & Bedeutung \\
\hline$r$ & Korrelationskoeffizient nach Pearson \\
\hline$r^{2}$ & Bestimmtheitsmaß \\
\hline $\bar{x}$ & Mittelwert \\
\hline * & multipliziert mit \\
\hline / & dividiert durch \\
\hline$\triangleq$ & entspricht \\
\hline$\rho$ & Kornrohdichte $\left[\mathrm{g} / \mathrm{cm}^{3}\right]$ \\
\hline$\rho t$ & Trockenrohdichte $\left[\mathrm{g} / \mathrm{cm}^{3}\right]$ \\
\hline$\sigma_{1}$ & 1. Hauptspannung, vertikal [kPa] \\
\hline$\sigma_{2,3}$ & 2. bzw. 3. Hauptspannung,horizontal [kPa] \\
\hline
\end{tabular}




\section{Einleitung und Zielsetzung der Arbeit}

Am Begin des dritten Jahrtausends steht die globale Landwirtschaft vor großen Herausforderungen. Der Anstieg der Weltbevölkerung, insbesondere in den Schwellen- und Entwicklungsländern, hält unvermindert an und wird nach UNSchätzungen im Jahr 2075 mit 9,22 Mrd. Menschen ihren Höhepunkt erreichen (UN 2004). Bereits bis 2050 wird die Weltnahrungsmittelproduktion um mindestens $70 \%$ steigen müssen, um der wachsenden Nachfrage gerecht zu werden (FAO 2010). Hinzu kommt, dass der Landwirtschaft neben ihrer klassischen Funktion als Nahrungsmittelproduzent auch in immer stärkerem Maße die Rolle als Bioenergie- und Rohstofflieferant zukommt. Um diesen Anforderungen gerecht zu werden, muss die globale landwirtschaftliche Produktion hochproduktiv und zugleich nachhaltig erfolgen.

Der Boden ist das zentrale Element der landwirtschaftlichen Produktion: das Vorkommen von Böden ist begrenzt; er ist nicht vermehrbar oder wiederherstellbar. Weder kann der Produktionsfaktor Boden durch Arbeit oder Kapital, noch kann der Lebensraum Boden durch Steinwolle oder Nährlösung ersetzt werden. Seinem Erhalt und dem Erhalt seiner Ertragskraft kommt daher entscheidende Bedeutung bei der Sicherung der Welternährung für künftige Generationen zu. Die Gefahren, denen Böden ausgesetzt sind, sind vielfältiger Art und global unterschiedlich stark ausgeprägt: neben Erosion, Versauerung, Versalzung, Desertifikation und den Folgen des Klimawandels ist auch die Gefahr der Verdichtung von Böden in den vergangenen Jahren und Jahrzehnten verstärkt in den Fokus des Interesses gerückt. Unter Bodenverdichtung wird die sog. anthropogene Bodenverdichtung verstanden, also jener Vorgang, bei dem durch Bodennutzung und mechanische Auflasten der Anteil der mit Gas und Wasser gefüllten Poren reduziert wird, während gleichzeitig das Volumen der festen Phase zunimmt (SCHEFFER \& SCHACHTSCHABEL 2002). Wird der Boden so stark verdichtet, dass er nachhaltig in seinen Funktionen beeinträchtigt wird, so wird auch der Begriff Bodenschadverdichtung gewählt (BMVEL 2002). Insbesondere kritisch zu sehen ist die Verdichtung der tieferen 
Bodenschichten, da hier regenerative Prozesse nur über lange Zeiträume wirken können (HORN 2004).

Bodenschadverdichtungen beeinträchtigen die Produktions-, Lebensraum- und Regelungsfunktionen des Bodens (BMVEL 2002). Größe und Zusammenhang des Porensystems verringern sich, so dass der Gas- und Wasseraustausch zwischen den Bodenschichten behindert wird. Folgen können Staunässe, ein verstärkter Oberflächenabfluss und Bodenerosion sein (SCHEFFER \& SCHACHTSCHABEL 2002). Durch Bodenverdichtungen können Pflanzenwachstum bzgl. der Gesamtwurzellänge wie auch die maximale Durchwurzelungstiefe gehemmt werden (EHLERS ET AL. 2000). Verdichtete Böden weisen eine schlechtere Wasserführung auf und können das Pflanzenwachstum durch reduzierte Nährstoff- und Sauerstoffaufnahme hemmen (SOANE \& VAN OUWERKERK 1994) und zu Mindererträgen führen (DüRR ET AL. 1995).

Im Zuge des Strukturwandels in der Landwirtschaft und der verstärkten überbetrieblichen Nutzung von Maschinen sind Größe und Gewicht der landwirtschaftlichen Geräte im Laufe der jüngeren Vergangenheit stark gestiegen. Gleichzeitig werden mit technischen Maßnahmen wie Reifendruckregelsystemen, GPS-gestützten automatischen Lenksystemen, Hundeganglenkung, mehrachsigen Fahrzeugen oder solchen mit Raupenlaufwerken Wege beschritten, die die negativen Effekte hoher Maschinenlasten auf den Boden verringern. Ackerbauliche Entwicklungen, wie der verstärkte Einsatz einer konservierenden, d. h. nicht wendend-lockernden Bodenbearbeitung können ebenfalls einen positiven Einfluss auf die Stabilität von Böden gegenüber mechanischen Auflasten haben (HORN 1986).

Nach Ansicht von FLoWERS \& LAL (1998) können weltweit ca. 68 Mio. Hektar als durch Befahrungen verdichtet angesehen werden. Allein auf EU-Ebene schätzen VAN DEN AKKER \& CANARACHE (2001) das Ausmaß der von Bodenverdichtung degradierten Böden auf 33 Mio Hektar. In Deutschland wird nach einer neuen Studie des Umweltbundesamtes im Zweiten Bodenschutzbericht der Bundesregierung (BT-DRS 16/12658 2009) davon ausgegangen, dass $49 \%$ der Ackerfläche der Bundesrepublik unter feuchten Bodenbedingungen eine hohe mechanische Verdichtungsempfindlichkeit im Unterboden aufweisen. Für 
Nordrhein-Westfalen gehen WEYER \& BUCHNER (2001) davon aus, dass 40\% der Flächen im Bereich der Krumenbasis schadverdichtet sind.

Die Ausmaße von Bodenverdichtungen, sowie der Umfang ihrer möglichen Schäden, ökonomisch wie ökologisch, werden ebenso kontrovers diskutiert wie die Eignung verschiedener Methoden zu ihrer Erfassung und das Ergreifen entsprechender Maßnahmen zu einer frühzeitigen Gefahrenabwehr. Methodische Ansätze zur Erfassung und Bewertung von Bodenverdichtungen umfassen neben der Bodenansprache im Feld auch die Bestimmung von Korngrößen, Lagerungsdichten, Porenvolumina, Luft- und Wasserleitfähigkeiten sowie das Drucksetzungs- und Scherverhalten von Bodenproben im Labor (HARTGE \& HORN 1992). Bereits an der Aufzählung der verschiedenen relevanten Aspekte ist zu erkennen, dass es sich hierbei um zeit- und kostenintensive Verfahren handelt, die bei großflächigen Untersuchungen auf Regressionsmodelle sog. Pedotransferfunktionen angewiesen sind. Deswegen haben Methoden, die eine schnelle und arbeitsextensive Untersuchung größerer Flächen ermöglichen eine große Bedeutung, weil sie helfen können, die Effizienz bodenkundlicher Untersuchungen erhöhen. Penetrometer sind Instrumente, die sich für solche Zwecke als besonders geeignet erachtet werden (SUN ET AL. 2004, LAPEN ET AL. 2004, DEXTER ET AL. 2007). Sie erfassen den Widerstand eines Bodens gegenüber dem Ein- oder Durchdringen einer Sonde von definierter Form und Größe und erlauben auf diese Weise Aussagen über die Stabilität des Bodens. Mit der Einführung von GPS-gestützten Systemen in Forschung und praktischer Landwirtschaft erhalten Penetrometer eine noch größere Bedeutung. Neben ihrer derzeitigen Anwendung in der großflächigen Datenerhebung ist mittelfristig auch ihr Einsatz bei der teilflächenspezifischen Bodenbearbeitung denkbar.

Im Rahmen eines gemeinsamen Forschungsprojektes der Christian-AlbrechtsUniversität zu Kiel (CAU) und der Fachhochschule Südwestfalen, Agrarwirtschaft Soest (FH-SWF) sollte die mechanische Stabilität von Ackerböden bei unterschiedlichen Bodenbewirtschaftungsverfahren untersucht werden. Die Betreuung der vorliegenden Arbeit seitens der FH-SWF erfolgte durch Prof. Dr. Ludwig Volk. 
Der landtechnische Ansatz sah dabei die Erhebung von Penetrometerdaten in vertikaler und horizontaler Ebene in einem fein abgestimmten Versuchsdesign vor, um Aussagen über die Anwendbarkeit des Verfahrens bei der Untersuchung von verdichteten Böden gewinnen zu können.

Hieraus ergeben sich folgende Ziele für die vorliegende Arbeit:

- Entwicklung eines Horizontalpenetrometers zur Erfassung von Eindringwiderständen

- Feldeinsatz des Systems innerhalb eines geordneten Versuchsdesigns zur Untersuchung des Einflusses der Faktoren Standort/Bodenart (Löss, Geschiebemergel), Bodenbewirtschaftung (konservierend, konventionell), Radlast und Reifeninnendruck auf den horizontalen Eindringwiderstand

- Vergleich der gewonnen Daten mit Ergebnissen von Vertikalpenetrometer-Messungen und Versuch einer Bezugstellung - Evaluierung des Verfahrens anhand bodenkundlicher Daten des Projektpartners sowie eigener Daten in Bezug auf die räumliche Identifizierung von Bodenschadverdichtungen

- Ableitung von Handlungsempfehlungen vor dem Hintergrund einer guten fachlichen Praxis

Die Förderung des Projektes erfolgte aus Mitteln des Bundesministeriums für Verbraucherschutz, Ernährung und Landwirtschaft (BMVEL) über die Bundesanstalt für Landwirtschaft (BLE). 


\section{Stand der Forschung}

Wie SOANE \& VAN OUWERKERK (1994) aufzeigen, wurde das Phänomen der Bodenverdichtung und seiner negativen Auswirkungen auf die landwirtschaftliche Produktion bereits zu Beginn des 20. Jahrhunderts von mehreren Autoren erkannt und beschrieben. In Deutschland legte Walter Söhne mit seinen Arbeiten zum Thema Bodenverformung und Bodenverdichtung die Grundlagen für eine bodenkundliche und agrartechnische Erforschung und Diskussion. Söhne stellte bereits 1951 fest, dass der Abbau von Druckspannungen, wie sie durch mechanische Auflasten in den Boden eingetragen werden, im Boden unter eliptischen Lastflächen schneller erfolgt als unter kreisförmigen, und dass die Druckspannungen bei breiteren Reifen aber konstantem Kontaktflächendruck bis in tiefere Bodenschichten hinabreichen (SÖHNE 1951). Söhne nutzte zur grafischen Darstellung der Bodenspannungen das Modell der Druckzwiebel, in dem die Isobaren der Druckspannung, also die Bereiche des Bodens, die unterhalb einer mechanischen Auflast eine identische Belastung erfahren, bildhaft dargestellt werden. Diese Darstellung ist noch heute gebräuchlich. In weiteren Untersuchungen (SÖHNE 1953, SÖHNE 1956) leistete Söhne großen Beitrag zur umfassenden mathematischen Beschreibung der Modelle, indem er Überlegungen von BouSSINESQ (1885) und FRÖHLICH (1934) (zitiert in SÖHNE 1953 und ZINK 2009) aus der Baumechanik aufgriff und weiterentwickelte.

Eine zusammenfassende Darstellung der zahlreichen Untersuchungen, die seither zum Thema Bodenverdichtung und seinen Folgen durchgeführt wurden, findet sich u. a. bei HAMZA \& ANDERSON (2005) und RAPER (2005). Angesichts der außergewöhnlichen Bedeutung des Bodens nicht nur für die Landwirtschaft, sondern auch für viele weiteren Lebensbereiche ist die Fülle der wissenschaftlichen Arbeiten auf diesem Feld nicht überraschend - ebenso wie ihre teilweise kontroversen Ergebnisse. Wie von (SOANE \& VAN OUWERKERK 1994) angeführt, kann die Erforschung des Themas nur auf Basis eines multi-disziplinären Ansatzes erfolgen. Der nachfolgende Teil soll einen Überblick über die ver- 
schiedenen methodischen Ansätze zur Erforschung von Bodenverdichtungen geben:

GYSI ET AL. (1999) haben Versuche auf sandigem Lehm durchgeführt und dabei festgestellt, dass Befahrungen mit Kontaktflächendrücken von $130 \mathrm{kPa}$ bzw. $160 \mathrm{kPa}$ in den Bodentiefen von $32-37 \mathrm{~cm}$ und $52-57 \mathrm{~cm}$ keine Verdichtung verursachen. Auch HEUER ET AL. (2008) zufolge bleiben bodenphysikalische Veränderungen des Bodens bei einfacher Befahrung mit einem Köpfrodebunker (8 - $11 \mathrm{Mg}$ Radlast) auf die Tiefen bis $25 \mathrm{~cm}$ beschränkt. In der tieferen Bodenschicht von $40-45 \mathrm{~cm}$ kann hingegen keine negative Wirkung nachgewiesen werden. Messungen von KOCH ET AL. (2008) zeigten, dass wiederholtes Befahren eines Lössbodens bei Feldkapazität mit Radlasten von 7,8-11,7 Mg Porenzusammensetzung, Eindringwiderstand und Luftdurchlässigkeit in Bodentiefen von $40-45 \mathrm{~cm}$ negativ beeinflusst und druckempfindliche Unterböden potentiell gefährdet werden. Dieses Risiko ist nach den Autoren jedoch als räumlich (auf das Vorgewende) und zeitlich (auf nasse Erntejahre) begrenzt anzusehen und daher insgesamt als tolerierbar einzustufen. Das langjährig angewendete Bodenbearbeitungsverfahren (konventionell oder konservierend) hatte in dieser Studie keinen nennenswerten Einfluss auf die Ergebnisse.

Auch die Versuche von SCHÄFER-LANDEFELD ET AL. (2004) haben ergeben, dass Befahrungseffekte auf die relevanten bodenkundlichen Parameter des Oberbodens beschränkt bleiben. Im ohnehin schon stark verdichteten Bereich der Pflugsohle konnten nur geringfügige Veränderungen festgestellt werden, ebenso in dem darunter liegenden Bereich des Unterbodens. Auf einer vor den Befahrungen tiefengelockerten Fläche wurden hingegen aufgrund der Aggregatdestabilisierung deutlich abnehmende Porenvolumina und Luftleitfähigkeiten auch im Unterboden gemessen. Eine Tiefenlockerung kann daher durchaus kontraproduktiv sein, wenn die Befahrintensität nachfolgend nicht reduziert wird. Obwohl die Autoren keine Hinweise auf eine generelle Gefährdung der Unterböden bei Achslasten bis $20 \mathrm{Mg}$ erkennen konnten, haben sie die Möglichkeit einer additiven Wirkung auch geringer Belastungen nicht ausgeschlossen. 
Auch BRUNOTTE ET AL. (2008) kommen in ihren Untersuchungen zu der Schlussfolgerung, dass die Gefahr einer flächenhaften Bodenverdichtung im Unterboden aktuell nicht gegeben ist. Hierzu haben die Autoren in den Jahren 2002/2003 und 2006 die Verbreitung von Bodenschadverdichtungen auf Flächen in Südniedersachsen untersucht. Ihr Zustand war bereits von RUHM (1983) (zitiert in BRUNOTTE ET AL. 2008) aufgenommen worden, der in seinen Arbeiten sogar noch frühere Untersuchungsergebnisse aus den 1950er Jahren berücksichtigt hatte. Die Autoren stellten fest, dass die Schadschwellen der Kriterien Luftkapazität und gesättigte Wasserleitfähigkeit nur auf 10 von 47 untersuchten Flächen unterschritten wurden, wobei sich die Werte der Packungsdichten in einem unkritischen Bereich bewegten. Lediglich in Fahrgassen und auf Vorgewenden konnten Verdichtungen nachgewiesen werden. Erwähnenswert ist weiterhin, dass es sich bei den untersuchten Schlägen um solche handelte, deren Bodendichte und Porenvolumen sich im Zeitraum der Jahre 1952 bis 1983 unvorteilhaft entwickelt hatten, aber im weiteren Verlauf der Bewirtschaftung bis zu den Untersuchungen der Autoren eine Regeneration erfahren hatten. Die Autoren führten dies auf eine weniger intensive Bodenbearbeitung, günstige Befahrzeitpunkte aufgrund erhöhter Schlagkraft und die Einstellung eines niedrigeren Reifeninnendruckes zurück.

SCHWARK (2005) untersuchte den Status von 17 landwirtschaftlichen Flächen in Schleswig-Holstein und stellte hierbei fest, dass dichter gelagerte Böden im Bereich der Kernproduktionsflächen die Bodenfunktionen nicht beeinträchtigen. Ähnlich der vorher genannten Studien konnte der Autor bis auf schädliche Bodenverdichtungen im Bereich der Fahrgassen und der Vorgewende eine generelle Verdichtungsgefährdung durch den Einsatz landwirtschaftlicher Großtechnik nicht erkennen.

Eine stark gegensätzliche Meinung wird hingegen von einer Reihe weiterer Forscher vertreten, die in ihren Untersuchungen durchaus von einer Gefährdung der Unterböden ausgehen:

FAZEKAS (2005) konnte in Befahrversuchen mit einem $35 \mathrm{Mg}$ schweren Köpfrodebunker unabhängig vom langjährig angewandten Bodenbearbeitungsverfahren Spannungseinträge bis in Tiefen von $60 \mathrm{~cm}$ feststellen. Diese waren 
bei konservierender Bodenbewirtschaftung stärker ausgeprägt, da ihre Ausbreitungen nicht durch die Schutzwirkung einer Pflugsohle verhindert wurden. Die Flächen unter Pflug stellen dabei n. E. der Autorin ein instabiles Bodenstruktursystem dar, in dem die Pflugsohle bei einmaligem hohen Lasteintrag in unregelmäßig große Stücke auseinander brechen und dadurch eine Unterbodenverdichtung bei nachfolgenden Befahrungen fördern kann.

Die Versuche von SEMmEL (1993) haben ergeben, dass bei Befahrungen mit $4 \mathrm{Mg}$ Radlast Drücke von $100 \mathrm{kPa}$ noch in Bodentiefe von $60 \mathrm{~cm}$ entstehen können. Auch DANFORS (1994) konnte bei Achslasten von 8 - 10 Mg auf Einzelachsen bzw. $10-20 \mathrm{Mg}$ auf Tandemachsen eine signifikante Abnahme des Porenvolumens in $30-40 \mathrm{~cm}$ und $40-50 \mathrm{~cm}$ Bodentiefe feststellen. Die Schlussfolgerungen aus vorangegangenen Untersuchungen, die Achslasten auf $6 \mathrm{Mg}$ (Einzelachse) bzw. $8 \mathrm{Mg}$ (Tandemachse) zu begrenzen, können n. E. der Autoren trotz verbesserter Reifentechnologie aktuell nicht revidiert werden. HAKANSSON \& REEDER (1994) fordern ebenfalls eine Begrenzung des mechanischen Lasteintrages, da ihren Untersuchungen zufolge Druckspannungen von Achslasten über $10 \mathrm{Mg}$ bis in Tiefen von $1 \mathrm{~m}$ nachweisbar sind.

EHLERS ET AL. (2000) stellten bei Überfahrungsversuchen auf LössParabraunerde bei Feldkapazität Ertragsdepressionen, eine Abnahme der Gesamtwurzellänge sowie eine Reduzierung der maximalen Durchwurzelungstiefe fest, die in Abhängigkeit des angewandten Bodenbearbeitungsverfahrens und des Jahres unterschiedlich stark ausfielen. Verdichtungen des Bodengefüges wurden bei hoher Belastung (6-malige Überfahrt bei $5 \mathrm{Mg}$ Radlast) bis in eine Tiefe von $50 \mathrm{~cm}$ dokumentiert. Die Autoren folgerten, dass Bodenverdichtungen in schluffreichen Unterböden nur eine eingeschränkte Fähigkeit der Regeneration aufweisen. Eine Umstellung auf konservierende Bodenbewirtschaftung als alleinige Maßnahme betrachteten sie aber als nicht ausreichend.

Die Arbeit von EHLERS ET AL. (2000) wie auch jene von MäHNER (1999) wurden für ihre Methodik von SCHWARK (2005) und anderen kritisiert, da die Befahrungen nicht unter praxisnahen Bedingungen stattfanden, sondern Radlader und Kranwagen genutzt wurden, die über keine landwirtschaftliche Bereifung 
verfügten und deren Reifeninnendruck mit bis zu $880 \mathrm{kPa}$ nicht den Verhältnissen angepasst war. Auch die Ergebnisse anderer Untersuchungen sind auf praktische landwirtschaftliche Anwendungen nicht übertragbar: von HALVORSON ET AL. (2003) wurden Befahrungen mit einem Abrams Panzer durchgeführt, PYTKA ET AL. (2006) nutzen einen Militär-LKW. Die Nutzung dieser Methoden mag im jeweiligen Einzelfall zielführend gewesen sein, sie bedürfen aber der Berücksichtigung bei der Ableitung von Handlungsempfehlungen. Ebenfalls kritisch muss ein methodischer Ansatz von BECHER (2005) bewertet werden, der bei der Betrachtung von Auswirkungen von Reifenbreite und Radstand auf die Druckverteilung unter landwirtschaftlichen Fahrzeugen die Annahme einer Radlast von $15 \mathrm{Mg}$ bei einer Reifenbreite von nur $30 \mathrm{~cm}$ zugrunde legt. Dieses muss als nicht praxisgerecht angesehen werden.

Die genannten Fälle verdeutlichen, dass eine Berücksichtigung der Methoden bei der Versuchsdurchführung wie bei der Analyse - bei der Bewertung der Ergebnisse eine große Bedeutung hat. Daher soll kurz auf die wesentlichen Untersuchungsmethoden zur Erfassung von Bodenverdichtungen eingegangen werden.

Das Konzept der Vorbelastung wurde von HORN \& FLEIGE (2003) vorgestellt und beruht auf Erkenntnissen der Baugrundmechanik. Es geht davon aus, dass der Boden bis zu einem bestimmten Maße mechanisch belastbar ist und bis zu einem horizontspezifischen kritischen Wert mit elastischer Verformung reagiert. Erst wenn die Auflast diesen Vorbelastungswert überschreitet, wird die mechanische Stabilität des Bodens überschritten und plastische Veränderungen können zu Beeinträchtigungen der Bodenfunktionen führen. Je höher eine bereits in der Vergangenheit erfolgte Belastung war, desto höher liegt auch der Vorbelastungswert eines Bodens, d. h. er kann gegenüber einem unbelasteten Boden relativ stärker belastet werden, bis eine weitere plastische Verformung und Bodenverdichtung einsetzt.

Das Konzept der Vorbelastung ist in der wissenschaftlichen Gemeinde etabliert, aber nicht unumstritten. SCHNEIDER \& SCHRÖDER (2005) geben zu bedenken, dass es sich bei der Methode um quasi-statische Labor-Messungen mit seitlich verhinderter Druckausbreitung handelt, die dynamische Prozesse, wie sie unter 
Praxisbedingungen im Feld anzutreffen sind, nicht abbilden. SCHWARK (2005) führt an, dass die Methode eine Reihe von Unsicherheiten beinhaltet, u. a. die Zeitdauer der Belastung der Laborprobe und die Ungenauigkeit bei der Abschätzung der Druckfortpflanzung. In einer datenintensiven Validierung des Konzeptes Vorbelastung kommen VORDERBRÜGGE \& BRUNOTTE (2011) zu dem Schluss, dass die Ergebnisse der Methode nur schwer reproduzierbar, statistisch nicht ausreichend abgesichert und Mess- und Schätzwerte nicht kongruent sind. In seiner Bewertung verschiedener methodischer Ansätze zur Identifikation von Schadverdichtungen hebt CRAMER (2006) jedoch die Eignung des Modells der Vorbelastung hervor, um einen wirkungsvollen Beitrag zum Bodenschutz zu leisten.

Als weiteres Konzept zur Erfassung von Bodenverdichtungen ist das Indikatormodell nach LEBERT ET AL. (2004) zu nennen, welches sowohl Bodenfunktionen als auch den Zustand des Bodengefüges berücksichtigt (BRUNOTTE ET AL. 2008). Hierbei werden Schadschwellen für die Kriterien Luftkapazität, gesättigte Wasserleitfähigkeit und Packungsdichte definiert, die alle überschritten sein müssen, um eine Bodenverdichtung zu identifizieren. CRAMER (2006) und LeBERT ET AL. (2004) stellen jedoch auch fest, dass derzeit noch keines der Modelle als vollständig anzusehen ist.

Auf die Konzepte der Schadverdichtungsgefährdungsklassen nach PETELKAU ET AL. (2000), des Druckbelastungsquotienten nach PAUL (2004) (beide zitiert in CRAMER 2006) u. a. soll im Rahmen dieser Arbeit nicht eingegangen werden, vielmehr bezieht sich der Autor der vorliegenden Arbeit auf Ergebnisse der erstgenannten Modelle.

Für den effektiven Einsatz der beschriebenen Methoden ist es notwendig, Bodengruben an repräsentativen bzw. aussagekräftigen Feldpunkten zu setzen. Hier können Untersuchungen, wie sie in der vorliegenden Arbeit durchgeführt wurden, wertvolle Hilfestellung geben. Die Erfassung des Widerstandes, den der Boden einer Sonde bei ihrem Eindringen entgegensetzt, ist ein transparentes Verfahren, um schnell Aussagen über Bodeneigenschaften auf einer Fläche treffen zu können. Der Einsatz solcher Penetrometer-Sonden erfolgt in der landwirtschaftlichen Praxis ebenso wie im Rahmen wissenschaft- 
licher Fragestellungen. Nach LUNNE ET AL. (1997) wurden Penetrometer in der Form, wie wir sie heute kennen, erstmalig 1932 in den Niederlanden eingesetzt. In den 1940er Jahren wurden sie bereits bei wissenschaftlichen Versuchen angewendet, um vertikale Eindringwiderstände zu erfassen und Effekte von Bodenbearbeitungsmaßnahmen zu untersuchen. Um den Eindringwiderstand als eine Funktion der Bodentiefe darstellen zu können, erfassen moderne Vertikalpenetrometer neben dem Eindringwiderstand auch die Eindringtiefe, Geräte der neuesten Generation darüber hinaus auch die GPS-Position. Weiterentwicklungen sehen eine Sensorkombination zur gleichzeitigen Erfassung des Bodenwassergehaltes vor (SUN ET AL. 2004).

Penetrometer wurden für verschiedenen Anwendungen entwickelt: neben dem Einsatz in der agrarwissenschaftlichen Forschung wurden sie auch für geologische Erkundungen (bis in eine Tiefe von mehr als $100 \mathrm{~m}$ ), für Sondierungen von Fluss- oder Meeresböden (LUNNE ET AL. 1997) aber auch für die Untersuchung der Mondoberfläche im Rahmen der Apollo-Missionen 15 und 16 eingesetzt (NASA 2001).

Der Messwert, den ein Penetrometer ausgibt, hängt neben den Bodeneigenschaften auch entscheidend von der verwendeten PenetrometerSonde, speziell von ihrem Durchmesser und dem Öffnungswinkel des Sondenkonus ab. Um eine Vergleichbarkeit von Messergebnissen zu gewährleisten, müssen Messungen mit identischen Sonden durchgeführt werden. Hierzu gibt es eine Vielzahl von Standards, über die LUNNE ET AL. (1997) einen Überblick geben. Die gebräuchlichsten stammen von der American Society of Agricultural and Biological Engineers (ASABE), vormals American Society of Agricultural Engineers (ASAE) und ASTM International (vormals American Society for Testing and Materials). Die ASAE sieht einen Konusdurchmesser von $12,83 \mathrm{~mm}$ oder $20,27 \mathrm{~mm}$ und einen Öffnungswinkel von $30^{\circ}$ vor (ASAE 1999), während von ASTM International eine Penetrometersonde mit einem Konus von $60^{\circ}$ und eine Eindringgeschwindigkeit von $2 \mathrm{~cm} / \mathrm{s}$ festlegt werden (ASTM 2004). Letzeres sieht auch das Niederländische Institut für Normung (NEN) in der NEN-NORM 5140: 1996 vor. Die letztgenannten Standards scheinen im Rahmen der europäischen Forschung die gebräuchlicheren (z. B. 
Cramer 2006, Ehlers et Al. 2000), auch weil große Anbieter von Penetrometern wie die Fa. Eijkelkamp sich an dieser entsprechenden Norm orientieren. Der Eindringwiderstand wird neben der im Boden vorherrschenden Spannungszustand und der Kegelgeometrie auch von der Bodenreibung und der Vorschubgeschwindigkeit beeinflusst (LEBERT ET AL. 2004). Die Autoren weisen außerdem auf die Änderung des Spannungstensors durch die Sonde selbst hin, die unberücksichtigt bleibt und stellen das Fehlen einer vollständigen mechanischen Theorie fest, die die Bedeutung der einzelnen Einflussfaktoren auf den Eindringwiderstand formuliert. CHUNG ET AL. (2004) betonen jedoch die Besonderheit des Verfahrens, Messwerte in großer Datendichte in situ erheben zu können. Diese Daten sollen als die Grundlage für weitere Bodenuntersuchungen oder Bodenbearbeitungsmaßnahmen angesehen werden. RAPER (2005) weist darüber hinaus auf die gute Vergleichbarkeit der Eindringwiderstände bei unterschiedlichen Bodentypen hin.

Neben Penetrometersonden, die den vertikalen Eindringwiderstand erfassen, wurden im Zuge erweiterter technischer Möglichkeiten auch Geräte für den Einsatz am Schlepper zur Erfassung des horizontalen Eindringwiderstandes entwickelt. Durch die Verfügbarkeit über globale GPS-gestützten Navigationstechnik (vgl. Sharpe ET AL. 2000, HATCH ET AL. 2002) können die gewonnen Daten mit Raumbezug als Flächendaten dargestellt und ein guter Überblick über die flächenhafte Ausprägung des aufgenommenen Kennwertes gewonnen werden.

Horizontalpenetrometer unterscheiden sich bezüglich ihres konstruktiven Ansatzes und äußerer Merkmale wie der verwendeten Sondenform oder der Anzahl der Sonden.

LEBERT ET AL. (2004) berufen sich auf frühe Entwicklungen von Einrichtungen zur horizontalen Erfassung der Eindringwiderstände von LINDNER (1964) und Petelkau (1989). Lüth (1993) (zitiert in Lebert et AL. 2004) entwickelte ein Horizontalpenetrometer, dass in modifizierter Form auch in späteren Arbeiten von WeIBBACH (1994), WILDE (2000) und SCHWARK (2005) genutzt wurde. Bei dem System kommen konusförmige Sonden zum Einsatz, die in $20 \mathrm{~cm}$ und $40 \mathrm{~cm}$ Tiefe Messwerte in einer Frequenz von $10 \mathrm{~Hz}$ aufnehmen können. 
WEIBBACH nutzte das Gerät, um Aussagen über den Verdichtungszustand von Fahrgassen auf ackerbaulich genutzten Flächen zu erheben. ScHWARK erhob mit dem Horizontalpenetrometer großflächig Daten und stellte sie anschließend in GIS-Karten dar. Der Autor konnte zeigen, dass in den Bereichen des Vorgewendes und der Fahrgassen erkennbar höhere horizontale Eindringwiderstände sowohl in $20 \mathrm{~cm}$ als auch in $40 \mathrm{~cm}$ Messtiefe identifiziert werden können.

Sun ET AL. (2006) und Schulze Lammers ET AL. (2007) setzten in Feld- und Laborversuchen ein Horizontalpenetrometer ein, das über einen kombinierten Sensor zur Erfassung von Eindringwiderstand und Bodenfeuchte verfügt. Die Entwicklung beruht auf der Übertragung des Messprinzips eines kombinierten Vertikalpenetrometer-Sensors (SUN ET AL. 2004). Die Systemgrenzen des horizontal arbeitenden Penetrometers liegen in einer geringen Arbeitstiefe von maximal $20 \mathrm{~cm}$ und in der Nutzung von nur einem konusförmigen Sensor. Die Daten zeigen, dass Bereiche, die zuvor mit einem Fendt GT380 Schlepper 5, 10 bzw. 20 mal befahren wurden, identifiziert werden konnten. Daten einer quantitativen Analyse der Eindringwiderstände in Bezug auf die Belastungsintensität sind nicht genannt. Einen Einfluss der Messtiefe $(10 \mathrm{~cm}, 15 \mathrm{~cm}$, $20 \mathrm{~cm}$ ) auf die Daten konnten die Autoren nicht feststellen.

Auch BöLENIUS ET AL. (2006) führten Messungen mit konischen Sensorsonden an einem Horizontalpentrometer in drei Tiefen auf einem Lehmboden in Schweden durch. Die Autoren konnten nachweisen, dass die Eindringwiderstände in $10 \mathrm{~cm}, 30 \mathrm{~cm}$ und $50 \mathrm{~cm}$ Bodentiefe unterschiedlich ausgeprägt sind. Die beiden unteren Messtiefen zeigten dabei eine ähnliche Ausprägung in der Fläche. Außerdem konnte eine Korrelation zwischen den erhöhten Eindringwiderständen in diesen Tiefen und den Ertragsdepressionen von Sommergerste hergestellt sowie eine tiefere Durchwurzelung in Bereichen mit geringen Eindringwiderständen festgestellt werden. Dieses deckt sich mit Beobachtungen von EHLERS ET AL. (2000).

Neben Systemen, die einen Konus als Sondenspitze nutzen, gibt es Ansätze, die über prismenförmige Scharspitzen für die Bodendurchdringung verfügen. CHUNG ET AL. (2003) konstruierten ein Horizontalpenetrometer, das mit fünf 
prismenförmigen Sensorspitzen im Abstand von 10,2 cm mit einem Öffnungswinkel von $60^{\circ}$ versehen ist und bis in eine Tiefe von $50 \mathrm{~cm}$ arbeiten kann. Das System lieferte bei Labor- und Freilanduntersuchungen auf unterschiedlichen Böden signifikante Korrelationen zwischen dem horizontalen Eindringwiderstand und den Parametern Lagerungsdichte und Bodenwassergehalt (CHUNG ET AL. 2004). Der Einfluss der Vorschubgeschwindigkeit unterhalb eines kritischen Wertes von $1,5 \mathrm{~m} / \mathrm{s}$ wurde als nicht signifikant ermittelt. Zwischen horizontalen und vertikalen Eindringwiderständen konnten positive Korrelationen $\left(r^{2}=0,5-0,6\right)$ ermittelt werden.

Auch SIEFKEN ET AL. (2005) konnten in Feldversuchen mit der Eigenentwicklung eines Horizontalpenetrometers eine gute Korrelation $\left(r^{2}=0,76\right)$ zwischen vertikalem und horizontalem Eindringwiderstand feststellen. Das System erfasste den Widerstand dreier Keile (Höhe $=10 \mathrm{~cm}$, Breite $=1,9 \mathrm{~cm}$, Öffnungswinkel $=45^{\circ}$ ), die ab Bodenniveau einen Messwert für jeweils $10 \mathrm{~cm}$ Bodentiefe aufnehmen. Die Keile sind an hintereinander liegenden Scharkörpern befestigt, die mit Dehnmessstreifen beklebt sind. Die Schare laufen in einer Furche durch den Boden, wobei mit dem ersten die Tiefe bis $10 \mathrm{~cm}$, mit dem zweiten die Tiefe bis $20 \mathrm{~cm}$ und dem dritten die Tiefe bis $30 \mathrm{~cm}$ gemessen wird. Die Messungen ergaben eine gute Übereinstimmung zwischen vertikalen und horizontalen Eindringwiderständen, und es konnte des Weiteren festgestellt werden, dass Geschwindigkeiten zwischen 0,45 m/s und 2,24 m/s keinen signifikanten Einfluss auf die Ergebnisse hatten. Für die horizontalen Datensätze wurden insgesamt geringere Varianzen ermittelt.

ALIHAMSYAH ET AL. (1990) entwickelten ein Ein-Sensor-System mit prismatischer Sonde ( $30^{\circ}$ Öffnungswinkel, $362 \mathrm{~mm}^{2}$ Grundfläche), das nachfolgend von CHUKWU \& BOWERS (2005) modifiziert und um zwei weitere Schare ergänzt wurde. Erstgenannter Autor fand beim Vergleich unterschiedlicher Scharformen heraus, dass prismenförmige Sonden im horizontalen Einsatz Vorteile gegenüber konusförmigen bieten, da sie keine Bruchzone in vertikaler Dimension (oberhalb und unterhalb des Sensors) hervorrufen und somit oberflächennäher eingesetzt werden können. Die Autoren konnten in Labor-Untersuchungen in homogenisiertem und definiert verdichtetem Boden feststellen, dass das System in der Lage war, unterschiedlich dicht gelagerte Böden und Boden- 
schichten zu differenzieren. Des Weiteren kamen sie zum Ergebnis, dass die Daten gut mit vertikalen Penetrometerdaten korrelierten $\left(r^{2}=0,76\right)$.

HALL \& RAPER (2005) entwickelten eine alternative Möglichkeit, um Aussagen über den Verdichtungszustand verschiedener Bodenschichten zu treffen, ohne die Zahl der Messsonden erhöhen zu müssen: eine einzelne prismenförmige Sonde wurde an einem vertikal oszilierenden Schar montiert, um auf diese Weise Messtiefen bis $35 \mathrm{~cm}$ beproben zu können. Mit der Methode gelang es den Autoren, dichter lagernde Bodenschichten zu identifizieren. Ferner ließen die Daten den Schluss zu, dass horizontale Messwerte des Systems weniger stark auf Änderungen des Bodenwassergehaltes reagieren als vertikale Vergleichsmessungen. Aufgrund der Ergebnisse stellten sie fest, dass diese Methode für die Erfassung von Bodenverdichtungen geeigneter ist als ein Vertikalpenetrometer.

Ein weiteres alternatives Konzept stellen SHARIFI ET AL. (2007) vor, die einen Scharkörper mit seitlichen Klappen („flaps“) versehen haben, auf deren Rückseiten Dehnmessstreifen verklebt wurden. Das System erwies sich in Labor- und Feldversuchen als geeignet, den Einfluss unterschiedlicher Reifeninnendrücke auf die Verdichtungswirkung im Boden bis $40 \mathrm{~cm}$ Tiefe zu erfassen.

Eine vollständige Übersicht über Sensoren zur Erfassung von Bodenverdichtungen, ihre Systematik und theoretische Hintergründe finden sich in HEMmAT \& ADAMCHUK (2008). In einem weiteren Artikel erweitern ADAMCHUK ET AL. (2004B) die Übersicht auf Sensorsysteme, die zur Untersuchung vielfältiger Aspekte von Böden genutzt werden. Neben den besprochenen mechanischen Systemen werden hier elektrische/elektromagnetische, optische/radiometrische, akustische/pneumatische sowie elektrochemische Sensorsysteme beschrieben. Die Autoren erkennen im der Zusammenführung einzelner Sensorsysteme („sensor fusion“) ein großes Potential für künftige Entwicklungen. Angesichts der zunehmenden Akzeptanz einer Präzisionslandwirtschaft gehen die Autoren ferner von einer Anpassung der Systeme an die Bedürfnisse der praktischen Landwirtschaft aus. 
Im Hinblick auf die guten Ergebnisse, die bei der Bodenbeprobung sowohl mit Vertikal- als auch mit Horizontalpenetrometern erzielt werden können, sehen methodische Entwicklungen dieses Verfahrens eine Verrechnung beider Messgrößen vor. Sie stellt neben der technischen Weiterentwicklung der Systeme eine weitere Möglichkeit dar, die Aussagekraft von Messdaten zu erhöhen. HARTGE (1986) greift das Konzept des Ruhedruckkoeffizienten oder Ruhedruckbeiwertes $\mathrm{K}_{0}$ auf, das in der Baugrundmechanik beschrieben ist und auch aktuell noch weiterentwickelt wird (z. B. MICHALOWSKI 2005). Hierbei werden die beiden Hauptspannungen (horizontal, vertikal) in Beziehung zu einander setzt. HORN ET AL. (1991) ermitteln mit Hilfe des Konzeptes den Ruhedruckkoeffizienten bei ungestörten Bodenproben im Labor. Auch FAZEKAS (2005) nutzt es erfolgreich, um bodenwirksame Effekte einer Befahrung anhand von Felddaten aus Überfahrungsversuchen $z u$ beschreiben. HEMMAT \& ADAMCHUK (2008) vermuten in Anlehnung an Untersuchungen von CHUNG ET AL. (2004) und anderen einen Zusammenhang zwischen vertikalen und horizontalen Messwerten in Bezug auf die Erfassung von Bodenverdichtungen. Ein Aufgreifen dieses Konzeptes kann daher den viel versprechenden Ansatz einer umfassenderen Zustandsbeschreibung von landwirtschaftlich genutzten Flächen bieten, als es bisher die Betrachtung eines einzelnen Datensatzes vermochte.

Die genannten und weitere Untersuchungen haben umfangreiche aber in ihrer Gesamtheit bei weitem keine eindeutigen Ergebnisse hervorgebracht bzgl. der Gefährdungswirkung schwerer Landmaschinen auf die Verdichtung von Boden, insbesondere von Unterböden. Ein umfassendes Konzept konnte bisher noch nicht etabliert werden. Seiner Vorsorgepflicht gerecht werdend, hat der Gesetzgeber aber bereits 1998 mit der Neufassung des Bundesbodenschutzgesetzes (BBODSCHG 1998) reagiert. Das Gesetz sieht vor, „nachhaltig die Funktionen des Bodens zu sichern" und "schädliche Bodenveränderungen abzuwehren“. Grundstückseigentümer sind verpflichtet, entsprechende Maßnahmen zu ergreifen. In weiteren Veröffentlichungen wurde das Handlungsprinzip der guten fachlichen Praxis (GFP) konkretisiert (BMVEL 2002). Es hat Eingang in zahlreiche Handlungsempfehlungen auf Länderebene gefunden, um die Gefährdung landwirtschaftlicher Flächen durch unsachgemäße Befahrung zu 
reduzieren (WeYer \& Boeddinghaus 2009, LUNG MV, Würfel ET AL.2002, BRANDHUBER ET AL. 2008, MUNLV 2004 u. a.). Seitens der Landmaschinenindustrie wurde ebenfalls auf eine mögliche Gefährdungswirkung reagiert, indem Rahmenbedingungen für einen Maschineneinsatz gemäß der GFP sowie Entscheidungshilfen benannt wurden (VDI 2007). 


\section{Material und Methoden}

\subsection{Versuchsstandorte}

Als Versuchsstandorte dienten die landwirtschaftlichen Versuchsgüter der an dem Projekt beteiligten Hochschulen. Am Standort Soest, Nordrhein-Westfalen konnten Versuchsflächen auf dem Versuchsgut Merklingsen der Fachhochschule Südwestfalen (FH-SWF) genutzt werden, während am Versuchsstandort Kiel, Schleswig-Holstein die Versuchsgüter Hohenschulen und Lindhof der Christian-Albrechts-Universität zu Kiel (CAU) in das Projekt eingebunden waren.

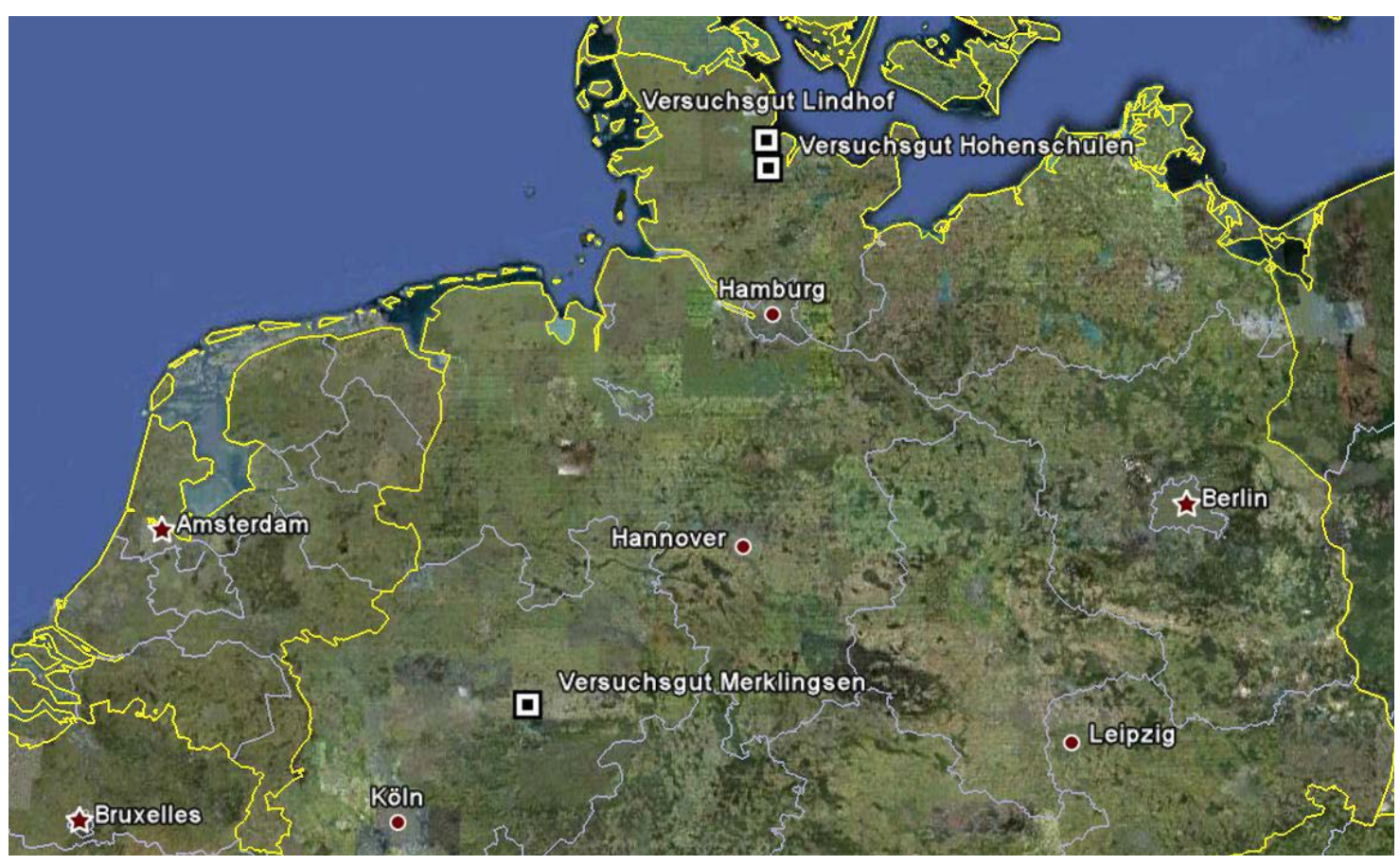

Abbildung 1: Geografische Lage der Versuchsgüter Merklingsen (NRW), Hohenschulen und Lindhof (jeweils SH) (Quelle: Google Earth).

In beiden Bundesländern stellten die Versuchsgüter jeweils zwei Flächen bereit, die sich in ihrer langjährigen Bodenbearbeitung (konventionell, konservierend) unterschieden. Unter konventioneller Bodenbearbeitung wird hierbei die jährlich wendende Lockerung des Bearbeitungshorizontes verstanden, wohingegen als konservierende Bodenbearbeitung die nicht-wendende Lockerung des Bodens definiert wird. Entsprechend ihrer Lage und Bodenbearbeitung werden die 
Versuchsflächen als $\mathrm{NRW}_{\text {konv }}, \mathrm{NRW}_{\text {kons, }}, \mathrm{SH}_{\text {konv }}$ und $\mathrm{SH}_{\text {kons }}$ bezeichnet. Die nachfolgende Einordnung der Versuchsstandorte in ihre naturräumlichen Haupteinheiten und deren Beschreibung erfolgt nach MEYNEN ET AL. (1962), sowie für die schleswig-holsteinischen Versuchsstandorte nach des Bundesanstalt für Geowissenschaften und Rohstoffe (BGR 2008). Eine detailliertere Beschreibung der einzelnen Versuchsflächen, insbesondere der bodenkundlich relevanten Kenngrößen, findet sich bei ZINK (2009).

\subsubsection{Soester Börde: Versuchsgut Merklingsen}

Die Soester Börde befindet sich am südlichen Rand der westfälischen Tieflandsbucht und bildet zusammen mit der Werl-Unnaer Börde im Westen und der Geseker Börde im Osten die Landschaft der Hellweg-Börden, benannt nach dem alten Handelsweg. Räumlich wird diese Landschaft nach Norden durch den Flusslauf der Lippe begrenzt, nach Osten durch die Paderborner Hochfläche, nach Westen vom Emscherland und im Süden durch das ansteigende Süderbergland mit dem angrenzenden Höhenzug des Haarstranges. Die Oberflächenkontur wechselt im Bereich der Hellweg-Börden nur großflächig und kann in großen Bereichen, insbesondere der Soester Börde, als weitgehend eben beschrieben werden. Der bodenbildende Hauptprozess im Bereich der Soester Börde war die nacheiszeitliche Ablagerung von Löss, die zumeist Parabraunerden ausbildete, welche aufgrund einer voranschreitenden Lessivierung zu mäßiger bis starker Pseudovergleyung neigen. 


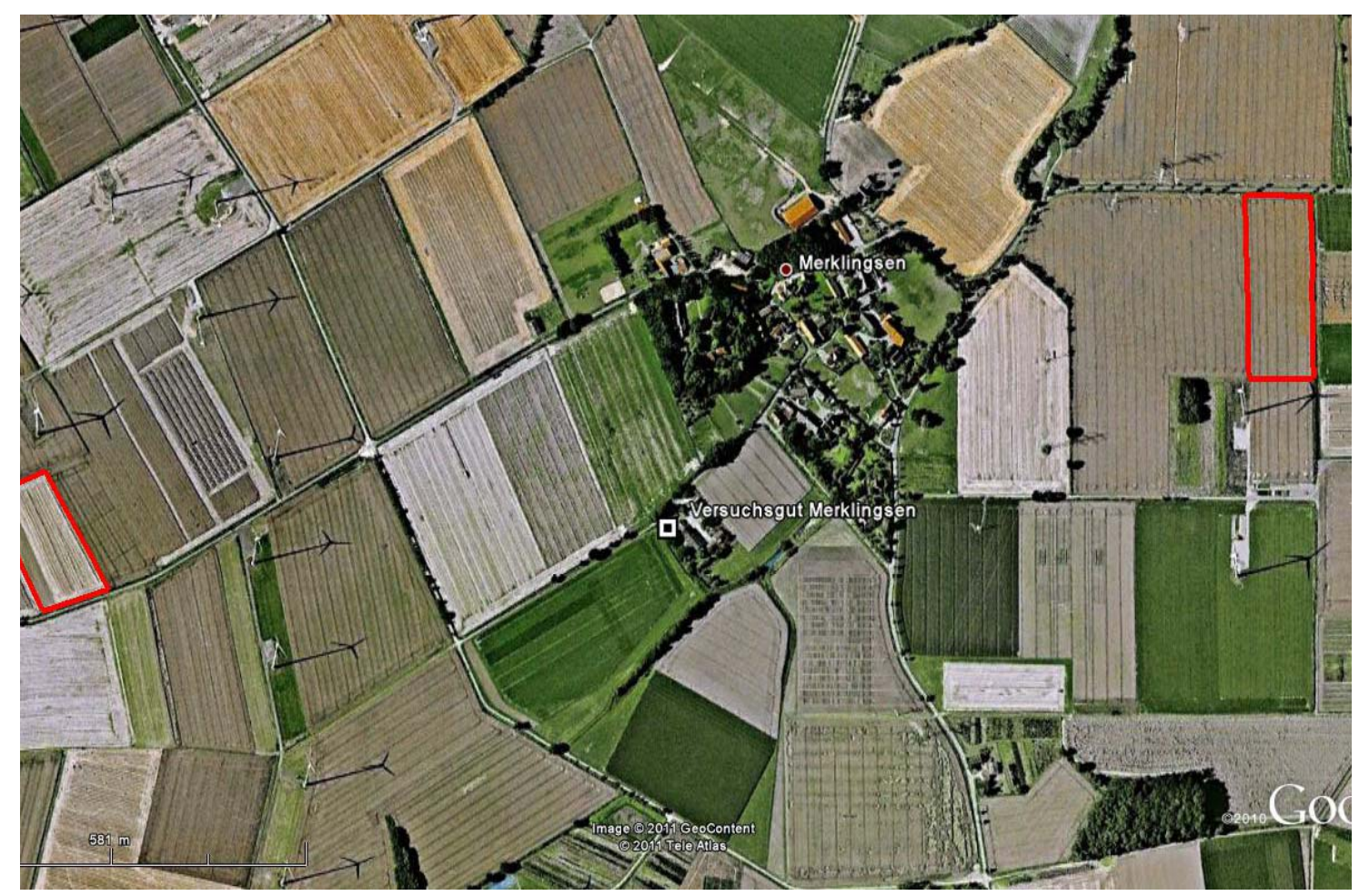

Abbildung 2: Versuchsgut Merklingsen (Mitte) mit den eingezeichneten Versuchsflächen

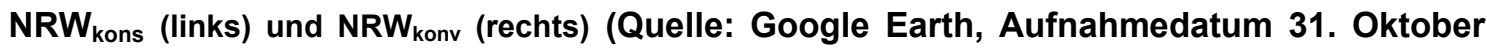
2004).

Das Versuchsgut Merklingsen befindet sich $10 \mathrm{~km}$ westlich der Stadt Soest auf einem Niveau von ca. $80 \mathrm{~m}$ üNN. Bei einer durchschnittlichen Jahrestemperatur von $9^{\circ} \mathrm{C}$ fallen im langjährigen Mittel $750 \mathrm{~mm}$ Niederschlag. Als vorherrschender Bodentyp ist eine Pseudogley-Parabraunerde aus Lösslehm mit einem Humusgehalt von $<2 \%$ und einer Bonität von 70 bis 75 Bodenpunkten zu finden. Die Hauptbodenart stellt ein mitteltoniger Schluff (Ut3) dar, mit einem Schluffanteil von 80 bis $85 \%$. Die Feldkapazität der ersten $100 \mathrm{~cm}$ beträgt $220 \mathrm{~mm}$.

Die Versuchsflächen weisen insgesamt eine geringe Hangneigung auf. Bei der Versuchsfläche $\mathrm{NRW}_{\text {kons }}$ handelt es sich um eine seit 1993 nicht-wendend bearbeitete Fläche im Versuchsgutbetrieb. Die langjährig wendend bearbeitete Fläche $\mathrm{NRW}_{\text {konv }}$ wurde für die Versuchsdurchführung zugepachtet. 


\subsection{2. Östliches Hügelland: Versuchsgüter Hohenschulen} und Lindhof

Schleswig-Holstein gliedert sich von West nach Ost in die Naturräume Marsch, Hohe Geest, Vorgeest und Hügelland. Das Hügelland wurde durch die vor ca. 115.000 Jahren einsetzende Weichsel-Eiszeit geprägt, deren maximale Ausdehnung bis zur Linie Flensburg - Rendsburg - Ahrensburg voranschritt. Die östlich dieser Linie gelegenen Gebiete wurden durch den sukzessiven Rückzug des Gletschereises und sedimentative Prozesse gekennzeichnet. Diese Prozesse spiegeln sich auch deutlich in der lebhaften Geländeform dieses Naturraumes wider. Das östliche Hügelland gehört zur Bodengroßlandschaft der Grundmoränenplatten und lehmigen Endmoränen im Jungmoränengebiet Norddeutschlands. In ihr liegen die Versuchsgüter Hohenschulen und Lindhof.

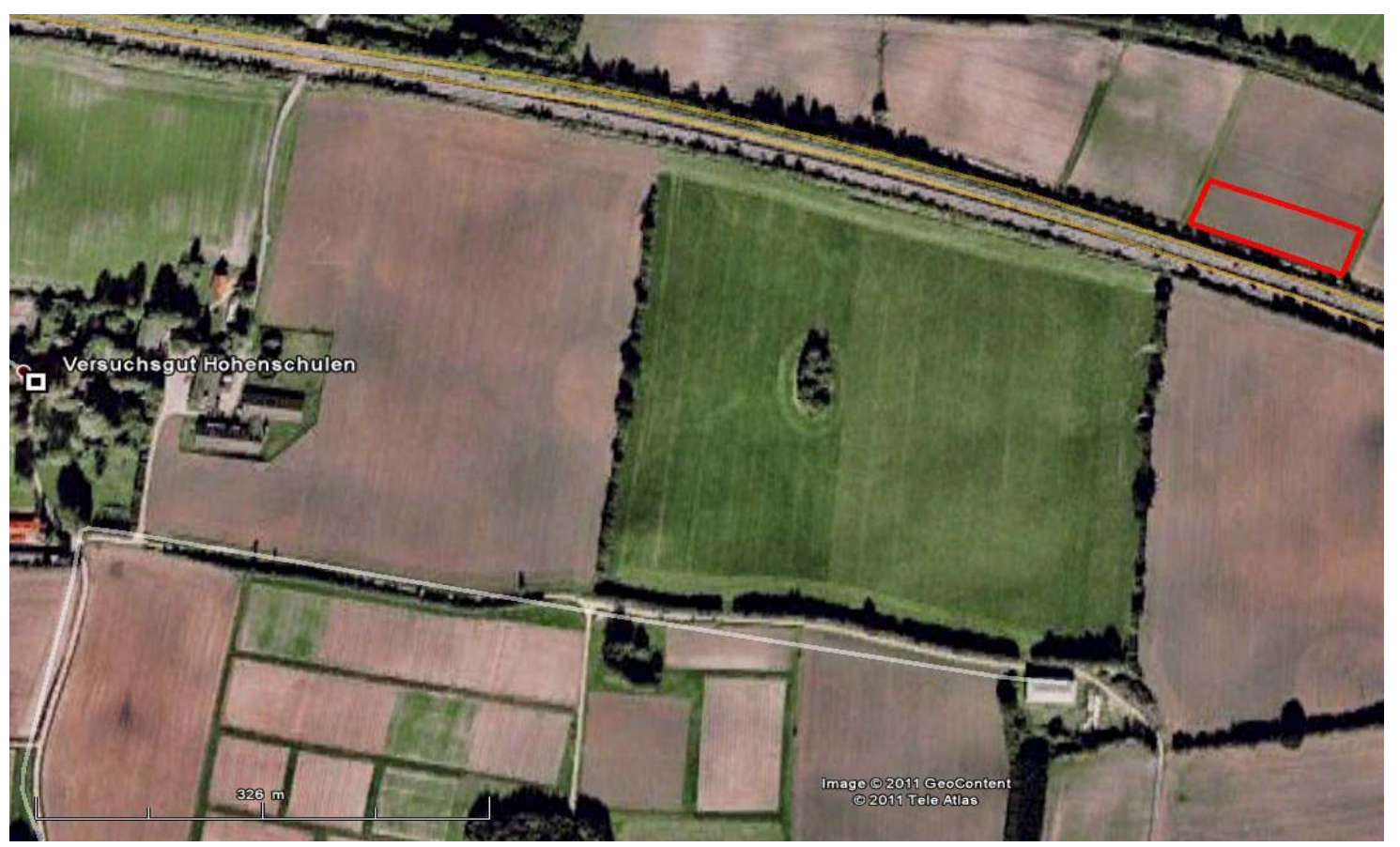

Abbildung 3: Versuchsgut Hohenschulen (links) mit den Teilflächen der Versuchsflächen $\mathrm{SH}_{\text {kons }}$ (Quelle: Google Earth, Aufnahmedatum 01. Januar 2000).

Das Versuchsgut Hohenschulen der CAU liegt im WestenseeEndmoränengebiet, etwa $15 \mathrm{~km}$ westlich von Kiel zwischen dem Westensee und dem Nord-Ostsee-Kanal. Die Flächen dieses Gebietes sind naturgemäß hügelig und insgesamt heterogener und deutlich steinreicher als jene am Standort NRW. Der auf der Versuchsfläche $\mathrm{SH}_{\text {kons }}$ vorherrschende Bodentyp ist 
eine Pseudogley-Parabraunerde aus Geschiebelehm. Als Bodenart findet sich ein stark sandiger Lehm in der Bodenschicht bis $28 \mathrm{~cm}$, nachfolgend bis in eine Tiefe von $70 \mathrm{~cm}$ dann ein lehmiger Sand. Die Jahresniederschläge sind in der Menge vergleichbar mit denen des nordrhein-westfälischen Versuchsstandortes, über das Jahr aber gleichmäßiger verteilt. Die Bonität der Versuchsgutflächen schwankt zwischen 40 und 60 Bodenpunkten, ebenso wie auch andere Bodenkennwerte eine heterogene Struktur aufweisen. Die Versuchsfläche $\mathrm{SH}_{\text {kons }}$ wird seit 1991 pfluglos bewirtschaftet und unterteilt sich in zwei Teilflächen, die ca. $200 \mathrm{~m}$ von einander entfernt liegen.

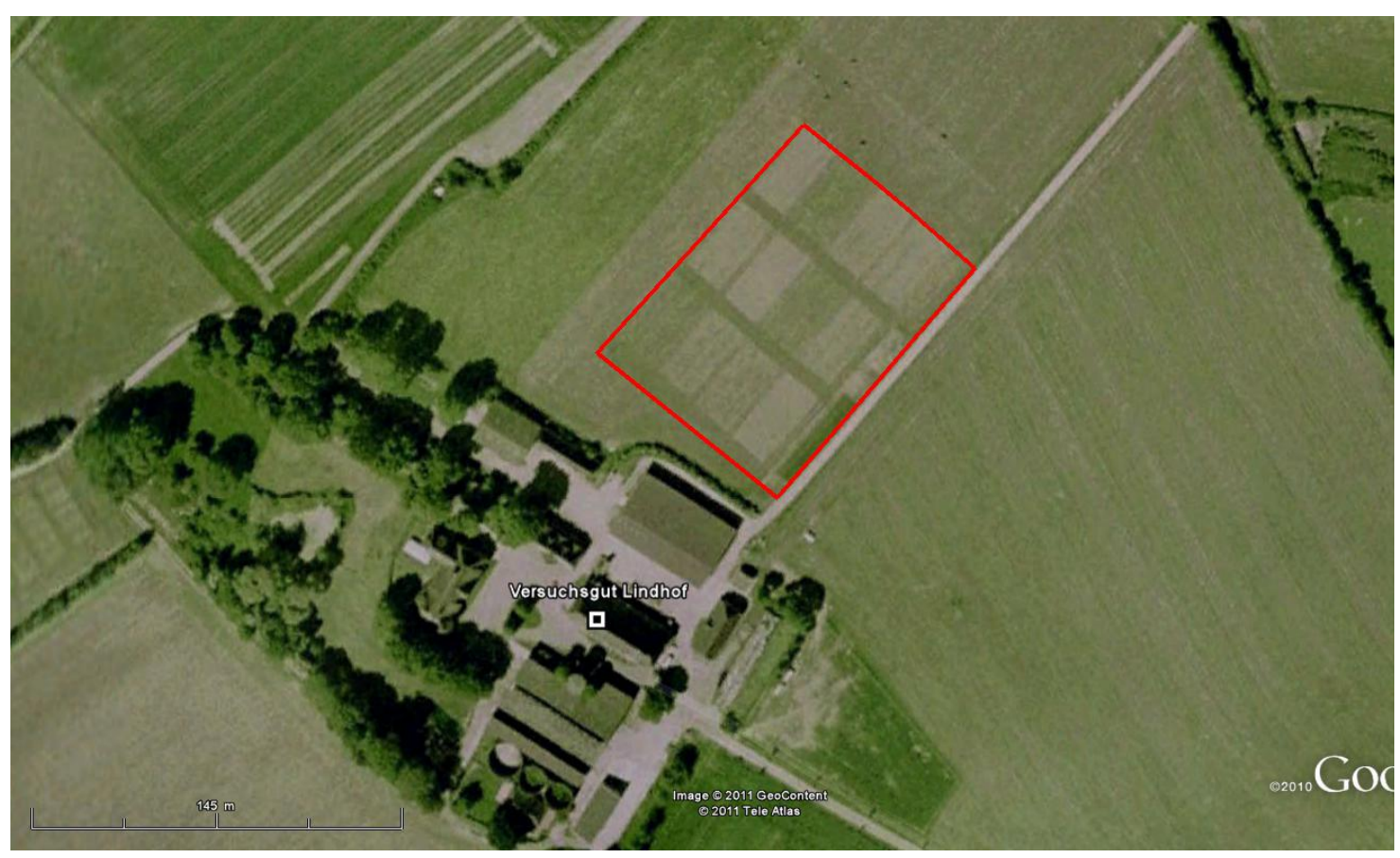

Abbildung 4: Versuchsgut Lindhof mit der angrenzenden Versuchsfläche $\mathrm{SH}_{\text {konv }}$ (Quelle: Google Earth, Aufnahmedatum 01. Januar 2000).

Am südlichen Ufer der Eckernförder Bucht, ungefähr $12 \mathrm{~km}$ süd-östlich von Eckernförde befindet sich auf der Halbinsel Dänischer Wohld das Versuchsgut Lindhof der CAU, auf dem die Versuchsfläche $S_{\text {konv }}$ liegt. Da die Bewirtschaftung des Versuchsgutes nach den Kriterien des ökologischen Landbaus erfolgt, wird aus entsprechenden ackerbaulichen Gründen eine wendende Bodenbearbeitung praktiziert. In der Fruchtfolge des Lindhofs stehen Kleegras, Sommergetreide, Körnerleguminosen, Kartoffeln und Winterweizen. Der vorherrschende Bodentyp ist eine Pseudogley-Parabraunerde aus Geschiebelehm, in dessen Ober- und nahen Unterboden sich mittel schluffige 
bis stark lehmige Sande finden, während im tieferen Unterboden mittel sandige Lehme vorherrschen. Die jährlichen Niederschläge liegen mit $680 \mathrm{~mm}$ etwas niedriger als auf dem $20 \mathrm{~km}$ südlicher gelegenen Versuchsgut Hohenschulen.

ZINK (2009) beschreibt die vier Versuchsflächen als typische Böden für die untersuchten Bodenregionen, auf denen die Vergleichbarkeit auf Bodenklassenniveau realisiert werden konnte.

\subsection{Versuchsaufbau und -durchführung}

\subsubsection{Faktoren und Faktorstufen}

Mit der Wahl der Versuchsflächen konnte neben einer unterschiedlichen Bodengenese in den entsprechenden Bodengroßlandschaften bereits auch das Bodenbearbeitungssystem als Faktor berücksichtigt werden. Der räumliche Rahmen, den diese vier Flächen darstellten, wurde für die Etablierung eines festen Versuchsdesigns genutzt. Dieses sah vor, an zwei Terminen im Jahr definierte mechanische Belastungen in Teilflächenbereiche einzubringen. Die Gesamthäufigkeit der Befahrungen umfasste in SH vier, in NRW fünf Termine. Alle Befahrungen wurden mit einer Geschwindigkeit von 1,4 m/s durchgeführt.

Bei der Erarbeitung des Versuchsdesigns wurden die Faktoren Radlast und Reifeninnendruck als die wesentlichen Parameter eines landwirtschaftlichen Radfahrwerks in Bezug auf seine Wirkung auf das Bodengefüge berücksichtigt. Die Befahrungen wurden mit dem Lastrahmen-System Movis (Motion Vehicle Information System) durchgeführt, das von einem praxisüblichen Schlepper gezogen wurde (vgl. 3.3.1). Aus einer dreifachen Abstufung des Faktors Radlast und einer zweifachen Abstufung des Faktors Reifeninnendruck ergaben sich sechs Belastungsvarianten (vgl. Tabelle 1). 
Tabelle 1: Faktorstufenkombination für den Reifen Michelin MegaXBib ${ }^{\circledR}$ 650/75 R32

\begin{tabular}{lll}
\hline Radlast $[\mathrm{Mg}]$ & Reifeninnendruck, niedrig $[\mathrm{kPa}]$ & Reifeninnendruck, hoch $[\mathrm{kPa}]$ \\
\hline 3,3 & $50 \mathrm{kPa}$ & $160 \mathrm{kPa}$ \\
6,3 & $160 \mathrm{kPa}$ & $250 \mathrm{kPa}$ \\
7,5 & $250 \mathrm{kPa}$ & $350 \mathrm{kPa}$ \\
\hline
\end{tabular}

Die Einstellung eines niedrigen und eines hohen Reifeninnendruckes kann hierbei als Annäherung an die gute bzw. schlechte landwirtschaftliche Praxis verstanden werden. Um eine differenzierte Betrachtung der einzelnen Faktoren zu ermöglichen, wurden die Reifeninnendrücke so gewählt, dass der hohe Reifeninnendruck einer Radlastvariante mit dem niedrigen Reifeninnendruck der nächst schwereren Radlastvariante identisch war. So unterscheiden sich benachbarte Varianten nur durch die Änderung von Reifeninnendruck oder Radlast, während der jeweils andere Faktor konstant bleibt. Den Zielen einer besseren Datenauswertung und Messsystembeurteilung war es geschuldet, dass die Reifeninnendrücke nicht in allen Varianten bis auf das vom Hersteller erlaubte Minimum abgesenkt wurden bzw. dieses in der Variante 3,3Mg@50kPa unterschritt (vgl. 3.3.1). Eine Übersicht der Varianten ab Herbst 2006, ihrer Bezeichnungen und Beschreibungen findet sich in Tabelle 2. Die Variante nur Schlepper, in der nur der für den Einsatz des Movis genutzte Zugschlepper die Befahrung durchführte, konnte aus Platzgründen nur auf $N R W_{\text {konv }}$ realisiert werden. 
Tabelle 2: Belastungsvarianten der Befahrungen ab Herbst 2006

\begin{tabular}{ll}
\hline Belastungsvariante & Beschreibung \\
\hline Kontrolle & unbefahren \\
nur Schlepper & Schlepper mit angepasstem Reifeninnendruck (50 bis $80 \mathrm{kPa}$ ) \\
(nur auf NRW $\mathrm{konv}$ ) & nach Verfügbarkeit \\
$3,3 \mathrm{Mg} @ 50 \mathrm{kPa}$ & $3,3 \mathrm{Mg}$ Radlast bei $50 \mathrm{kPa}$ Reifeninnendruck \\
$3,3 \mathrm{Mg} @ 160 \mathrm{kPa}$ & $3,3 \mathrm{Mg}$ Radlast bei $160 \mathrm{kPa}$ Reifeninnendruck \\
$6,3 \mathrm{Mg} @ 160 \mathrm{kPa}$ & $6,3 \mathrm{Mg}$ Radlast bei $160 \mathrm{kPa}$ Reifeninnendruck \\
$6,3 \mathrm{Mg} 250 \mathrm{kPa}$ & $6,3 \mathrm{Mg}$ Radlast bei $250 \mathrm{kPa}$ Reifeninnendruck \\
$7,5 \mathrm{Mg} @ 250 \mathrm{kPa}$ & $7,5 \mathrm{Mg}$ Radlast bei $250 \mathrm{kPa}$ Reifeninnendruck \\
$7,5 \mathrm{Mg} @ 350 \mathrm{kPa}$ & $7,5 \mathrm{Mg}$ Radlast bei $350 \mathrm{kPa}$ Reifeninnendruck \\
$\mathrm{GEO}$ & Sonderfläche Geophysik \\
& $6,3 \mathrm{Mg}$ Radlast bei $250 \mathrm{kPa}$ Reifeninnendruck \\
& Spur-an-Spur-Befahrung auf $15 \mathrm{~m}$ * 15 m mit entsprechend \\
& mehrfacher Überrollung durch den Zugschlepper \\
\hline
\end{tabular}

Nicht alle Varianten konnten unmittelbar zu Versuchsbeginn realisiert werden: Die Varianten 7,5Mg@250kPa und 7,5Mg@350kPa konnten technisch erst zum zweiten Termin im Herbst 2006 umgesetzt werden. Eine Einbindung dieser Varianten erfolgte, indem auf $\mathrm{NRW}_{\text {konv }}$ unbefahrene Kontrollparzellen in die Befahrungssystematik aufgenommen wurden (und somit in Summe eine Befahrung weniger aufweisen als Varianten mit 3,3 Mg und 6,3 Mg Radlast), bzw. indem auf $\mathrm{NRW}_{\text {kons }}$ Parzellen der Varianten mit 3,3 Mg bzw. 6,3 Mg mit den höherlastigen Varianten überlagert wurden. Des Weiteren unterschieden sich die Belastungsvarianten des Frühjahres 2006 aufgrund technischer Umbaumaßnahmen am Movis geringfügig von jenen der späteren Messtermine (vgl. Tabelle 3).

Da die Befahrungen am Standort SH erst im Herbst 2006 begannen, konnte eine Berücksichtigung aller Varianten ab Versuchsbeginn sichergestellt werden. 
Tabelle 3: Faktorstufenkombinationen für Michelin MachXBib 650/75 R38 (nur NRW Frühjahr 2006)

\begin{tabular}{lll}
\hline Radlast $[\mathrm{Mg}]$ & Reifeninnendruck A $[\mathrm{kPa}]$ & Reifeninnendruck B $[\mathrm{kPa}]$ \\
\hline $3,2 \mathrm{Mg}$ & $50 \mathrm{kPa}$ & $160 \mathrm{kPa}$ \\
$6,5 \mathrm{Mg}$ & $180 \mathrm{kPa}$ & $250 \mathrm{kPa}$ \\
\hline
\end{tabular}

Neben der für die Etablierung des Versuchsdesigns benötigten Fläche wurden an jedem Standort kleinere Teilflächen ausgewiesen, die für gesonderte Untersuchungen zur Verfügung standen. Die Sonderflächen Geophysik wurden auch für agrartechnische Untersuchungsmethoden genutzt. Die Befahrung dieser Flächen wurde mit der Variante 6,3Mg@250kPa in der Weise durchgeführt, dass auf einer Fläche von $15 \mathrm{~m} * 15 \mathrm{~m}$ Spur an Spur gefahren wurde.

\subsubsection{Versuchsdurchführung}

Entsprechend der Belastungsvarianten wurde für jede Versuchsfläche ein Versuchsdesign erstellt, wie es in Abbildung 5 am Beispiel der Fläche NRW konv $_{\text {va }}$ dargestellt ist. Auf dieser Fläche konnte die vollständige Durchführung aller Varianten in drei Blöcken erfolgen. Auf den Flächen $\mathrm{NRW}_{\text {kons }}, \mathrm{SH}_{\text {konv }}$ und $\mathrm{SH}_{\text {kons }}$ musste die Anzahl der Blöcke aufgrund räumlicher Begrenzungen mit Ausnahme der Varianten $\mathrm{K}_{0}$ und 7,5Mg@350kPa auf zwei Wiederholungen begrenzt werden. Die Parzellen lagen im Kernproduktionsbereich der Flächen und wurden je Termin mit drei Spuren in einfacher Überrollung quer zur Hauptbearbeitungsrichtung befahren. Alte Fahrgassen lagen in den Zwischenblockbereichen, die zur Änderung des Reifeninnendruckes genutzt wurden. Eine Änderung der Radlasten in diesem Bereich wurde aufgrund der damit verbundenen starken Befahrintensität als nicht vertretbar erachtet und auf den befestigten Hofflächen durchgeführt. 


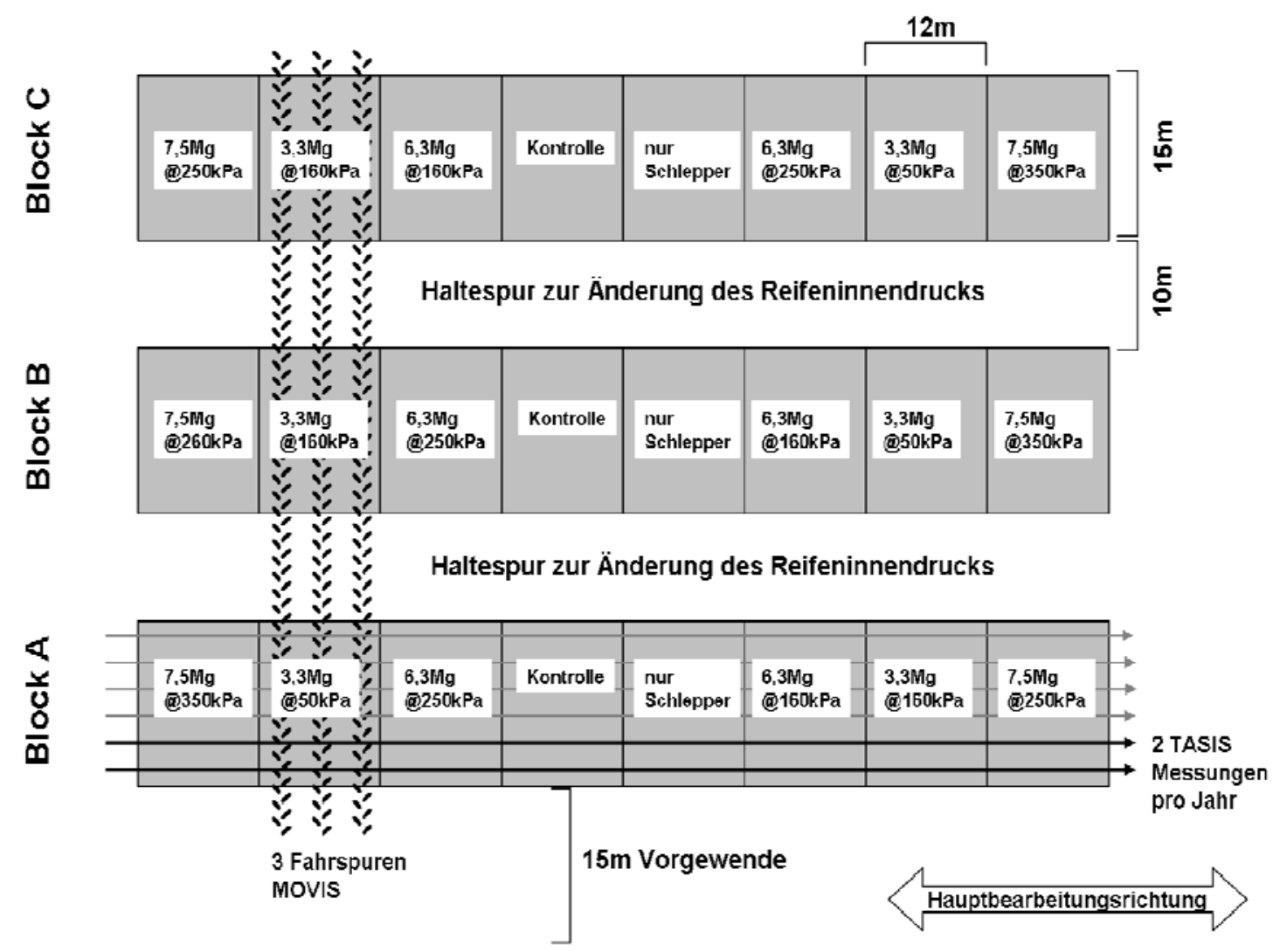

Abbildung 5: Versuchsdesign am Beispiel der Fläche NRW $_{\text {konv. }}$

Die Termine der Befahrung fanden nach Absprache zwischen den Arbeitsgruppen statt. Angestrebt waren Bodenverhältnisse nahe der Feldkapazität, um Effekte im Boden hervorzurufen, deren Nachweis die unterschiedlichen Messmethoden erbringen sollten. Beginnend in NRW im Frühjahr 2006 fanden bis zum Frühjahr 2008 jeweils zwei Befahrungen pro Jahr statt. Die Versuchsflächen in SH wurden ab Herbst 2006 befahren, so dass innerhalb des Versuchszeitraums vier bzw. fünf Befahrungen durchgeführt wurden. Die Fahrspuren wurden vom Institut für Geophysik der CAU mit einer Genauigkeit von $2-3 \mathrm{~cm}$ eingemessen. Vor und nach jeder Befahrung fanden Untersuchungen mit unterschiedlichen Messmethoden statt, wie sie in 3.3 detailliert beschrieben sind.

Die angestrebte praxisnahe Bewirtschaftung der Flächen während des Versuchszeitraums erfuhr geringe Einschränkungen durch Mulch- oder Bodenbearbeitungsmaßnahmen, die im Sinne der Versuchsanstellung notwendig waren, um eine geordnete Versuchsdurchführung sicherzustellen. 
Für $\mathrm{NRW}_{\text {kons }}$ und $\mathrm{NRW}_{\text {konv }}$ erfolgte, mit Ausnahme der Bodenbearbeitung, eine synchronisierte Bewirtschaftung über den Versuchszeitraum. Eine Übersicht über die Bewirtschaftungsmaßnahmen der einzelnen Flächen ab 2000 findet sich in Tabelle A 6.

\subsection{Messsysteme und Messwerterfassung}

\subsubsection{Movis: Mechanischer Lasteintrag unter definierten Bedingungen}

Die Befahrungen wurden auf allen Flächen mit dem Messsystem Movis (Motion Vehicle Information System) durchgeführt, das von einem Schlepper gezogen wird. Das Chassis des Movis bildet ein halbseitig offener Trägerrahmen aus verschweißten Vierkant-Stahlrohre, in dessen front- und heckseitigen Bereichen Registergewichte aus Gussbeton eingesetzt werden können, um die Radlast zu variieren (siehe Abbildung 6). Im geschlossenen Seitenelement des Trägerrahmens ist eine mittig durchtrennte Mähdrescherachse verbaut, deren Getriebeeinheit zuvor demontiert worden war. Der auf ihr montierte Reifen befindet sich zentral in dem U-förmigen Rahmen und überrollt den unbefahrenen Bereich zwischen rechten und linken Rädern des Zugschleppers. Die Anhängung erfolgt über die Unterlenker des Zugschleppers. Sie ist auf einer zentrale Lenkachse montiert und kardanisch aufgehängt. Bei Vorfahrt auftretende Zugkräfte wirken direkt auf eine Stahlplatte, auf der Dehnungsmessstreifen (DMS) verbaut sind. 


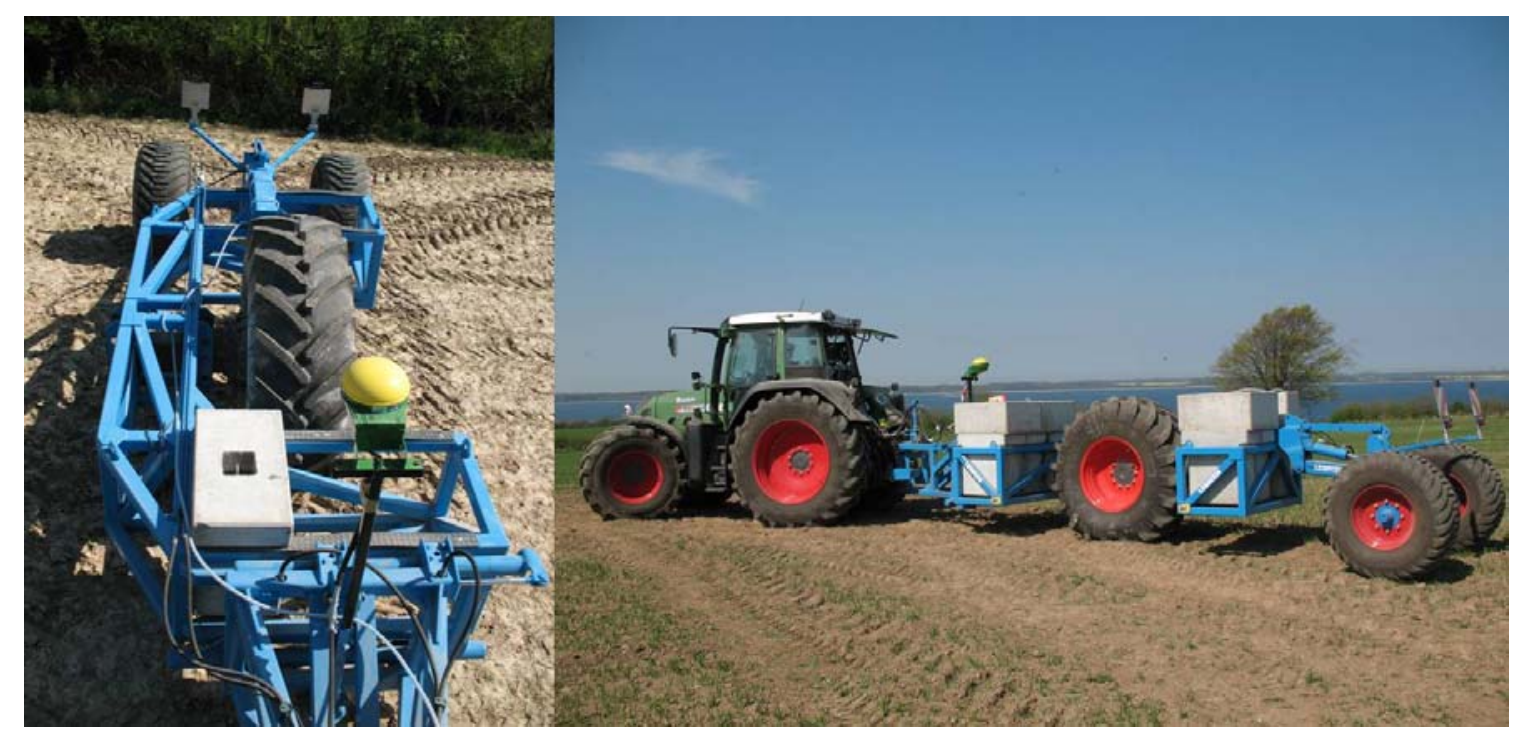

Abbildung 6: Movis (Motion Vehicle Information System) in diagonaler Draufsicht und Seitenansicht.

Am Heck des Movis ist eine Liftachse montiert, die über einen Zylinder hydraulisch abgesenkt werden kann. Dadurch wird das zentrale Messrad entlastet, um kürzere Transportstrecken zu bewältigen. Für die Versuchsdurchführung wird die Liftachse angehoben und das zentrale Rad wird mit der gesamten Masse des Movis belastet. Die Stabilisierung des Wagens erfolgt dann über einen seitlichen Hydraulikzylinder im Frontteil des MovIS.

Einzig für die Befahrungen am Standort NRW im Frühjahr 2006 wurde der Reifentyp Michelin MachXBib ${ }^{\circledR}$ 650/75 R38 verwendet. Er wurde für alle Befahrungen ab Herbst 2006 durch einen Michelin MegaXBib ${ }^{\circledR}$ 650/75 R32 ersetzt, der gegenüber dem zugkraftoptimierten MachXBib $^{\circledR}$ durch einen zusätzlichen Stahlgürtel für höhere Tragfähigkeiten ausgelegt ist und hohe zyklische Lasten abstützen kann, wie sie bspw. auch bei Mähdreschern vorkommen. Aufgrund dieser Eigenschaften kommt er vorrangig bei Erntemaschinen zum Einsatz. Die Dimension des Reifens wurde beibehalten, da sie vor dem Hintergrund begrenzter Transportbreiten bei Landwirten und Lohnunternehmern einen gängigen Kompromiss zwischen Straßen- und Feldfahrten darstellt. Der MegaXBib ${ }^{\circledR}$ ist gemäß Herstellerspezifikationen nicht für einen Reifeninnendruck von $<100 \mathrm{kPa}$ vorgesehen. Die Variante 3,3Mg@50kPa entspricht somit nicht den Spezifikationen des Herstellers und auch nicht der gängigen landwirtschaftlichen Praxis, stellt aber im Sinne einer 
bodenschonenden Befahrung durch abgesenkten Reifeninnendruck eine BestCase-Variante dar.

\subsubsection{Erfassung des vertikalen Eindringwiderstandes}

Der vertikale Eindringwiderstand wurde mit dem Penetrologger ${ }^{\circledR}$ der Firma Eijkelkamp (Giesbeek, Niederlande) erfasst. Bei diesem Gerät werden die Kraft und Messtiefe per DMS bzw. per integriertem Ultraschallsensor erfasst und in einen Datenspeicher geschrieben. Die Messungen wurden im Bereich des Stollenabdrucks durchgeführt Die Abdrucktiefe des Stollens wurde bei der Datenauswertung berücksichtigt. Je Parzelle wurden 18 Messungen durchgeführt - jeweils 3 pro Movis-Spur vor der Befahrung und 3 danach. Extremwerte der Einzelmessungen, etwa durch das Auftreffen der Sonde auf einen Stein, wurden manuell aus dem Datensatz gelöscht. Die Daten wurden anschließend mit der Hersteller-Software PenetroViewer ${ }^{\circledR}$ (Version 4.24) ausgelesen und konnten in Tabellenkalkulations- und Statistikprogrammen verarbeitet werden. Um das Datenvolumen zu reduzieren und die Grundlage für die Vergleichbarkeit von vertikalen und horizontalen Eindringwiderständen zu schaffen, wurden einzelne Messtiefenbereiche $(5-14 \mathrm{~cm}, 18-27 \mathrm{~cm}, 30-39 \mathrm{~cm}$ und $50-59 \mathrm{~cm}$ ) gebildet und die Messwerte innerhalb jeder einzelnen Messung für diese Bereiche gemittelt. Der für die Messungen verwendete Messkonus wies bei einer Grundfläche von $1 \mathrm{~cm}^{2}$ einen Öffnungswinkel von $60^{\circ}$ auf und wurde mit einer Eindringgeschwindigkeit von $2 \mathrm{~cm} / \mathrm{s}$ in den Boden eingeführt. Diese Methode entspricht der NEN-Norm 5140 des niederländischen Institutes für Normung (NEN 1996).

Zu allen Messzeitpunkten wurde der Bodenfeuchtegehalt mit einer FDR-Sonde in den Tiefen $20 \mathrm{~cm}$ und $40 \mathrm{~cm}$ sowie an der Bodenoberfläche erfasst. Die Sonde misst den Scheinwiderstand des Bodens, um mit Hilfe der Dielektrizitätskonstanten des Bodenwassergehalt zu bestimmen (SCHERZER ET AL. 1996). Je Versuchsfläche wurden zur Erfassung der Bodenfeuchte vier Messpunkte mit je fünf Messungen pro Bodentiefe beprobt. 


\subsubsection{TASIS: Erfassung des horizontalen}

\section{Eindringwiderstandes}

Zur Erfassung der horizontalen Eindringwiderstände wurde an der FH-SWF das Messsystem TASIS (Tactile Soil Information System) entwickelt. Als Konstruktionsbasis diente der Rahmen eines Tiefenlockerers, der über drei Aufnahmen für die Scharkörper verfügte. Die Aufnahmen wurden in den mittleren Bereich des Rahmens versetzt und anstelle der werksseitig montierten Lockerungsschare wurden eigene Scharkonstruktionen montiert (vgl. Abbildung 7).
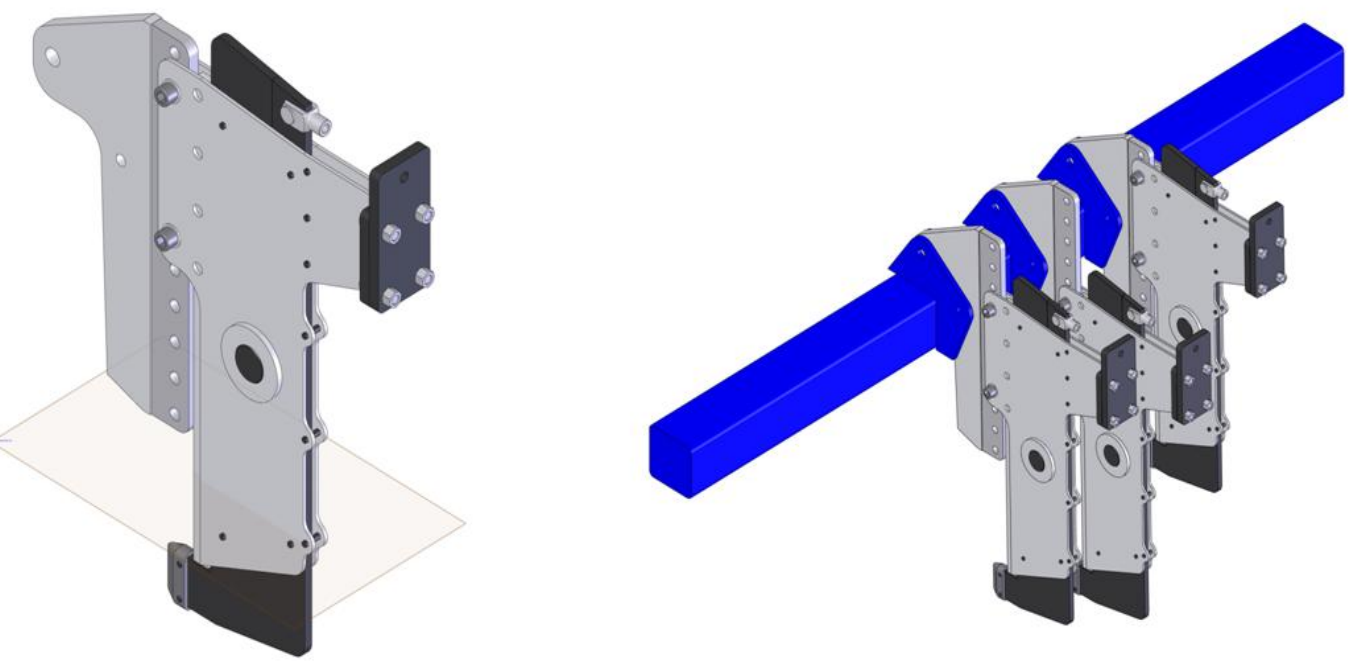

Abbildung 7: CAD-Zeichnung eines einzelnen Schares und der gesamten Schargruppe des Messsystems TASIS.

Die Scharkonstruktionen bestehen jeweils aus einem beweglichen inneren und einem starren äußeren Scharkörper (vgl. Abbildung 8). Der äußere Scharkörper ist über eine Lochschiene mit der Scharaufnahme am Rahmen fest verschraubt und kann in $65 \mathrm{~mm}$-Schritten in der Höhe verstellt werden. Die seitlichen Bleche sind $6 \mathrm{~mm}$ stark und im vorderen Bereich mit einem soliden Stahlvierkant verschweißt, der zu einem Grat (Öffnungswinkel $=45^{\circ}$ ) abgeschliffen wurde. Am oberen hinteren Ende des äußeren Scharkörpers ist eine Stahlplatte rechtwinklig verschraubt, die einen festen Bezugspunkt für einen DMSKraftaufnehmer bildet, der den äußeren ummantelnden Scharkörper mit dem 
inneren Scharkörper verbindet. An dessen unteren Ende befindet sich eine haftreibungsfreie Messsonde, die gegenüber dem äußeren Schar $70 \mathrm{~mm}$ vorständig ist und bei Vorfahrt den Boden horizontal durchdringt. Über einen $65 \mathrm{~mm}$ starken mittig platzierten Bolzen sind die Scharkörper verbunden. Um ihn kann sich das innere Schar drehen und die auf die Messsonde wirkenden Kräfte umlenken, so dass sie vom Kraftaufnehmer im oberen Bereich erfasst werden können. Als Kraftaufnehmer dienen in Abhängigkeit von der Arbeitstiefe Flachstähle von $1 \mathrm{~mm}, 2 \mathrm{~mm}$ oder $4 \mathrm{~mm}$ Stärke, die für Nennlasten von $5 \mathrm{kN}$, $10 \mathrm{kN}$ und $20 \mathrm{kN}$ ausgelegt sind und mit je zwei DMS beklebt wurden. Die Anordnung zweier DMS zu einer DMS-Vollbrücke bietet die größtmögliche Genauigkeit und weist eine hohe Kompensation gegenüber thermischen und mechanischen Störungen auf (HofFMANN 2004). Die Kalibrierung der DMSElemente wurde mittels geeichten Gewichten in Laboren der FH-SWF vorgenommen. Die Übereinstimmungen der gemessenen Ausgangsspannungen der Kraftaufnehmer mit den Gewichtselementen betrugen jeweils $R^{2}=0,99$.

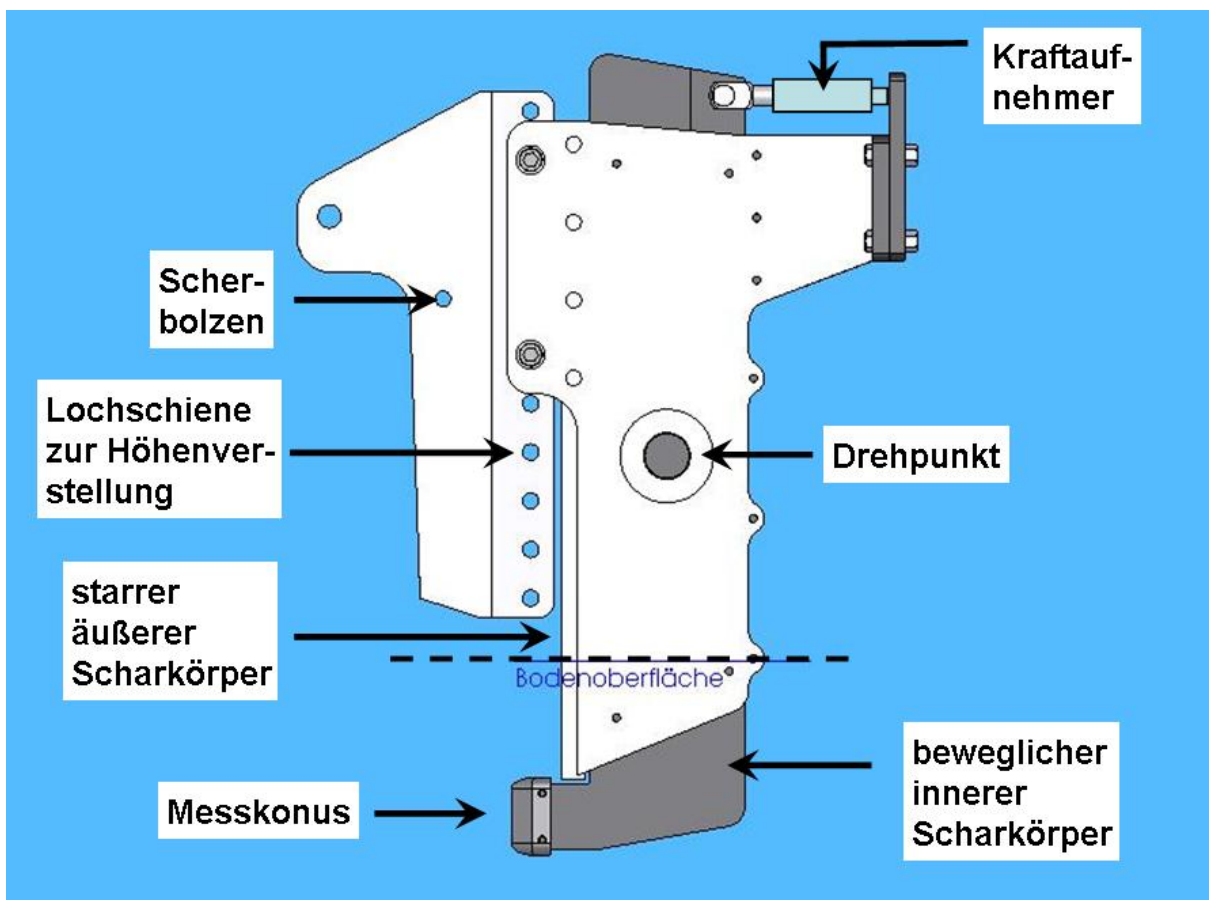

Abbildung 8: Scharkörper von TASIS mit seinen Funktionselementen in der Seitenansicht.

Die Messsonden von TASIS stellen einen Hybrid aus Prisma und Konus dar, die im $7 \mathrm{~cm}$ hohen Mittelteil einem Prisma gleichen und in den sich horizontal anschießenden Endstücken zu einem Konus mit $3 \mathrm{~cm}$ Durchmesser abflachen. 
Der Öffnungswinkel beträgt jeweils $60^{\circ}$, die Grundfläche $2.806 \mathrm{~mm}^{2}$. Die Schare sind in vertikaler Ausrichtung an dem Scharkörper montiert. Prismenförmige bzw. Hybrid-Sonden in vertikaler Ausrichtung bieten gegenüber konischen Sondenspitzen den Vorteil, dass mit weniger Sonden ein größerer Messbereich in der Vertikalen erfasst werden kann und die Gefahr einer Messung unterbzw. oberhalb einer plattenartigen Gefügestruktur - und somit deren NichtErfassung - minimiert wird. Des Weiteren sollte der Gefahr entgegen gewirkt werden, einen plattenartigen Verdichtungshorizont dadurch nicht zu erkennen, dass sich die in der Vertikalen stabilen Gefügeplatten beim horizontalen Durchdringen einer konischen Messsonde nach oben und unten verlagern bzw. brechen. Dadurch könnte der Sonde ein abfallender Spannungstensor im Boden vorauseilen, was eine Fehlinterpretation der Messwerte zur Folge hätte.

Die analogen Messsignale der DMS-Messbrücke werden über einen Messkraftverstärker BA-600 (Vishay Intertechnology Inc., Malvern, USA) verstärkt, bevor sie von einer 12-Bit-DAQ-Card NI-6024E (National Instruments, Austin, USA) digitalisiert und über den PCMCIA-Bus in den Laptop eingelesen werden. Zeitgleich mit den analogen Messdaten der DMS werden GPSKoordinaten über einen USB-Port verarbeitet, deren Bereitstellung über einen StarFire ${ }^{\circledR}$ GPS-Empfänger (John Deere, Moline, USA) mit einer Genauigkeit der Positionsbestimmung von 5 bis $10 \mathrm{~cm}$ erfolgte. Zur digitalen Erfassung und Synchronisation beider Datenströme wurde die Software LabView ${ }^{\circledR}$ (National Instruments, Austin, USA), Version 8.2 genutzt (vgl. Abbildung 9). 


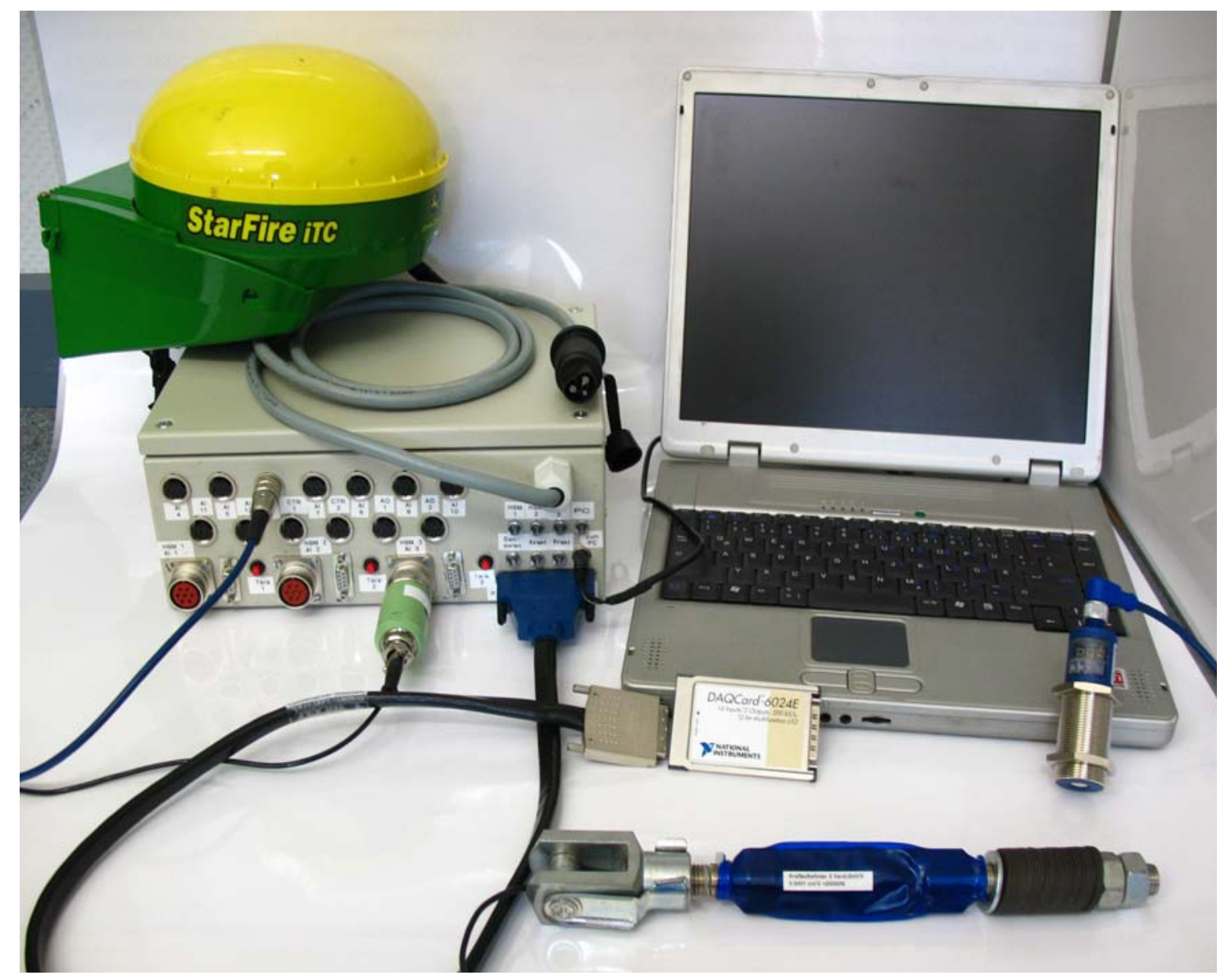

Abbildung 9: Einzelkomponenten des Messsystems TASIS.

TASIS sollte in der Versuchsanordnung kleinräumige Verdichtungsbereiche erfassen und trotzdem Aussagen auf einer gesicherten Datengrundlage zulassen. Hierfür war eine ausreichend große Basis analoger Messwerte und deren genaue Zuordnung zu den digitalen GPS-Daten erforderlich. Um eine möglichst genaue Auflösung der Daten zu erreichen, ohne die Genauigkeit des GPS-Signals zu gefährden, wurde eine geringe Fahrgeschwindigkeit von $0,27 \mathrm{~m} / \mathrm{s}$ gewählt. Die analoge Datenerfassung erfolgte bei $100 \mathrm{~Hz}$. Die GPSDaten wurden mit $5 \mathrm{~Hz}$ erfasst und von LabView ${ }^{\circledR}$ linear interpoliert.

Messungen auf den Versuchsflächen wurden mit TASIS einmal jährlich im Herbst nach erfolgten Befahrungen in zwei Wiederholungen durchgeführt und erfolgten in Hauptbearbeitungsrichtung, also quer zu den Movis-Fahrspuren. Sie wurden in einem Abstand von 2,50 m zueinander realisiert, so dass am Ende der 3-jährigen Versuchszeit eine vollständige Beprobung der $15 \mathrm{~m}$ breiten Versuchsparzellen stattgefunden hatte. Bereits beprobte Bereiche der 
Versuchsflächen wurden in nachfolgenden Jahren abermals erfasst, die gewonnenen Messdaten bei der Datenauswertung jedoch gesondert berücksichtigt. Dadurch konnten sowohl die Wirkungen der Befahrung als auch die Lockerungswirkung durch den Bodeneingriff der TASIS erfasst werden. Die Arbeitstiefen der drei Messsonden lagen in den Bereichen $5-14 \mathrm{~cm}, 18-27 \mathrm{~cm}$ und $30-39 \mathrm{~cm}$. Die Arbeitstiefenführung erfolgte über die Lagesteuerung der elektronischen Hubwerksregelung und wurde über einen Ultraschallsensor MIC +35/IU/TC (Microsonic, Dortmund) erfasst und als Messwert im Datenprotokoll erfasst.

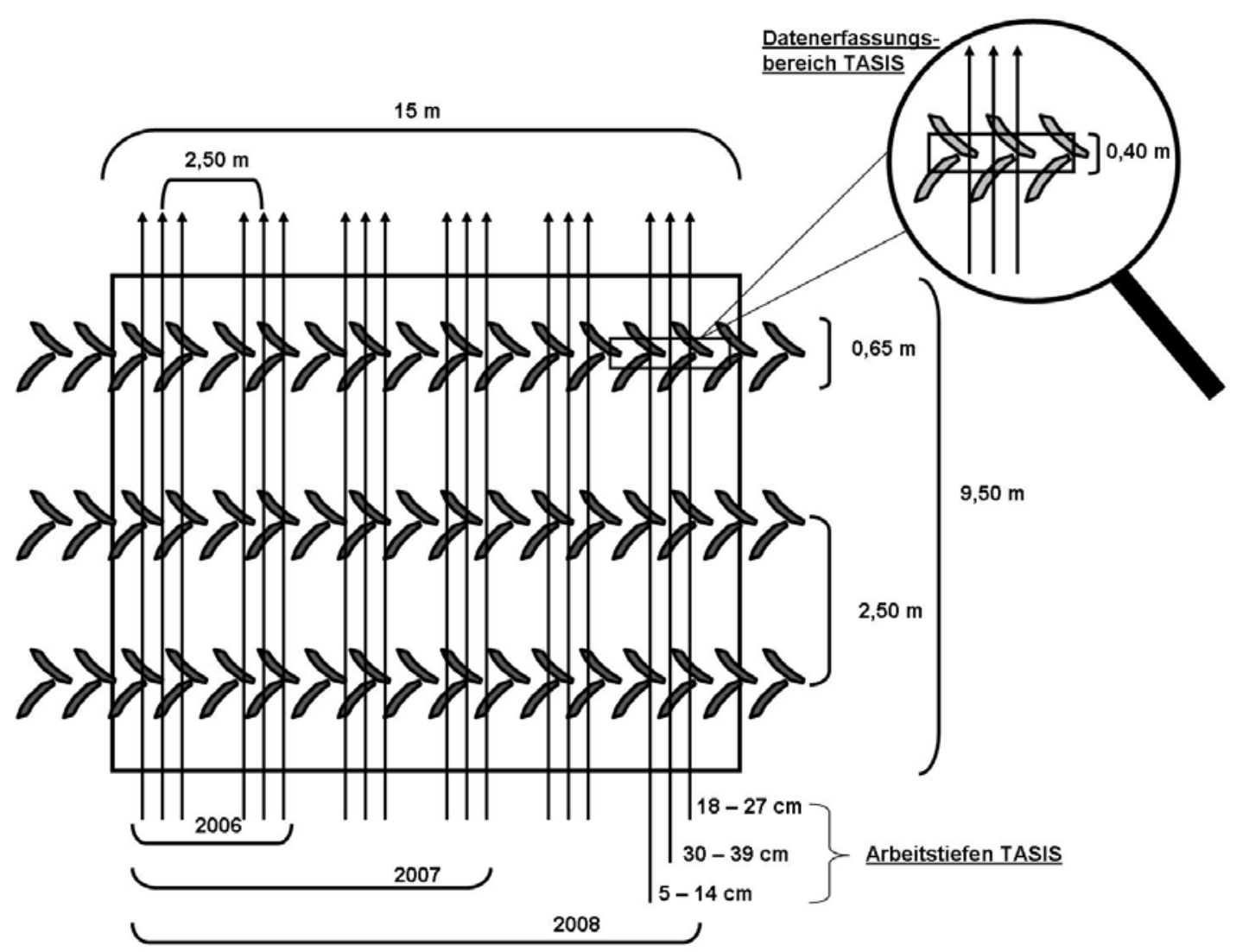

Abbildung 10: Darstellung des Beprobungsschemas innerhalb einer Versuchsparzelle.

Da für die spätere Datenauswertung lediglich die horizontalen Eindringwiderstände im Bereich der $65 \mathrm{~cm}$ breiten Fahrspuren berücksichtigt werden sollten, wurden mit Hilfe des GIS-Programms ArcView ${ }^{\circledR}$ (Version 3.2) jene Werte aus den TASIS-Datensätzen herausgefiltert, die im zentralen Bereich der Fahrspuren lagen. Die Breite dieses Datenpuffers wurde auf $40 \mathrm{~cm}$ 
festgelegt. Die Daten innerhalb dieses Bereiches einer jeden Fahrspur wurden gemittelt und flossen als ein Wert in die weitere Verrechnung ein.

\subsubsection{Bodenphysikalische Untersuchungen}

Nach Ablauf der Versuchslaufzeit wurden auf der Fläche $N R W_{\text {konv }}$ ergänzende bodenphysikalische Untersuchungen in den Bodentiefen $36-40 \mathrm{~cm}$ und $60-$ $64 \mathrm{~cm}$ durchgeführt. Beprobt wurden die unbefahrene Kontrollfläche, die innerhalb des Versuchsdesigns gelegene Variante 7,5Mg@350kPa und die Sonderfläche Geophysik. Die Beprobungen beinhalteten die Entnahme von Stechzylinderproben und Spatendiagnosen. Für die Stechzylinderuntersuchungen wurden Stechzylinder mit $100 \mathrm{~cm}^{3}$ Volumen gewählt, die vertikal mit 20 Wiederholungen pro Tiefe gezogen wurden. Die Stechzylinderentnahme fand im Juni 2008 statt, die Spatendiagnose erfolgte im Oktober 2008. Die Spatendiagnosen erfolgten entsprechend AG BODEN (2005), DIN 19682-10:1998 (DIN 1998A) und der maßgeblichen Information der Bayerischen Landesanstalt für Landwirtschaft (LFL 2005).

Bei der Stechzylinderuntersuchung wurden folgende Parameter im Labor untersucht:

- Bestimmung der Kornrohdichte ( $\rho$ ) nach DIN ISO 11508: 2002 (DIN 2002) mit Pyknometern

- Gravimetrische Bestimmung der Lagerungsdichte ( $\rho t$, Trockenrohdichte) durch Trocknung der Stechzylinder bei $105^{\circ} \mathrm{C}$ bis zur Gewichtskonstanz (HARTGE \& HORN 1992)

- Gesamtporenvolumen, berechnet aus der Lagerungsdichte des Bodens und der Kornrohdichte 
Das Gesamtporenvolumen wurde wie folgt berechnet:

$$
\begin{array}{ll}
G P V=(1-(\rho t / \rho)) * 100 \\
\text { GPV: } & \text { Gesamtporenvolumen } \\
\rho \mathrm{t}: & \text { Lagerungsdichte } \\
\rho: & \text { Kornrohdichte }
\end{array}
$$

Formel 1: Berechnung des Gesamtporenvolumens

Die Porengrößenverteilung wurde nach CRAMER (2006) an $100 \mathrm{~cm}^{3}$ Stechzylindern bestimmt. Die eingesetzten Drücke von 0,06 und 0,3 bar entsprechen den Wasserspannungen pF 1,8 und pF 2,5 bzw. den Äquivalentdurchmessern von $50 \mu \mathrm{m}$ (weite Grobporen) und $10 \mu \mathrm{m}$ (enge Grobporen). Der Feinporenanteil (0,2 $\mu \mathrm{m}$ Äquivalentdurchmesser) wurde an gestörten Bodenproben bei 15 bar entsprechend pF 4,2 ermittelt. Durch Differenzbildung wurde der Mittelporenanteil ermittelt.

Die Bestimmung der gesättigten Wasserleitfähigkeit (kf-Wert) erfolgte mittels Labor-Permeameter 09.02 (Eijkelkamp; Giesbeek, Niederlande) unter Anwendung der Darcy-Gleichung (CRAMER 2006 in Anlehnung an DIN 19683-9: 1998 (DIN 1998B)).

Die Bestimmung der Luftleitfähigkeit wurde mit dem Feldmessgerät PL 300 (UGT, Müncheberg) an Stechzylindern unter Anwendung der Darcy-Gleichung vorgenommen. Hierbei wurde der Messdruck als hydraulische Druckhöhe angesetzt und in cm Wassersäule (cm WS) gemessen (UGT 1999).

\subsubsection{Erfassung weiterer Parameter}

\section{Bodenwassergehalt}

Die volumetrischen Bodenwassergehalte wurden mit einer FDR-Sonde (Frequency Domain Response) an allen Terminen erfasst, an denen Vertikalpenetrometer, TASIS oder Movis eingesetzt wurden. Für die Penetrometermessungen wurde für jede Versuchsfläche an vier Messpunkten die Bodenfeuchte in drei Messtiefen $(0 \mathrm{~cm}, 20 \mathrm{~cm}, 40 \mathrm{~cm})$ mit je fünf Wiederholungen 
ermittelt. Zur Bestimmung der Bodenfeuchte bei Befahrung mit Movis wurden ab Frühjahr 2007 drei Messungen pro Fahrspur durchgeführt.

Der gravimetrische Wassergehalt wurde durch die von ZINK (2009) ermittelten Werte der mittleren Lagerungsdichten der jeweiligen Versuchsfläche errechnet:

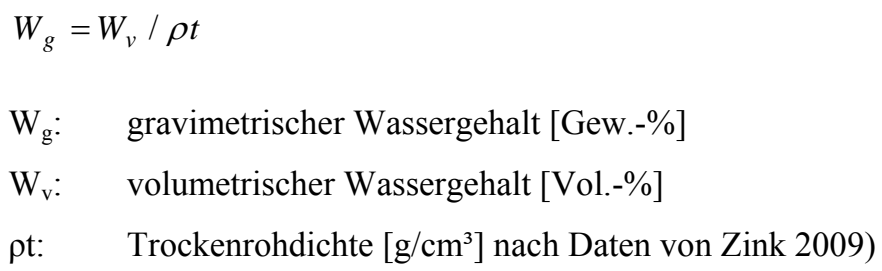

Formel 2: Berechnung des gravimetrischen Wassergehaltes

\section{Achslasten}

Die Radlasten am Movis wurden für jede Ballastierungsvariante mit einer mobilen Achslastwaage (Mayer, Tittmoning) erfasst. Dieses geschah zu Beginn des Projektes. Nach den durchgeführten Umbaumaßnahmen wurden die Messungen im Laufe des Projektes erneut durchgeführt, um Radlastveränderungen zu dokumentieren bzw. eine gleich bleibende Ballastierung sicherzustellen.

\section{Reifenkontaktflächen}

Die Kontaktflächen der einzelnen Belastungsvarianten wurden zu jedem Messtermin und für jede Versuchsfläche erfasst: An einer repräsentativen unbefahrenen Stelle der Fläche wurde das Messrad des Movis abgesenkt und gründlich mit Mehl umstäubt. Anschließend wurde das Rad ausgehoben und der Mehlabdruck aus senkrechter Perspektive photographiert. Ein Gliedermaßstab lag hierbei über die volle Breite im Bild, welches anschließend mit der Software Adobe Photoshop (7.0) ausgewertet wurde, indem der Mehlabdruck mit einem Polygon-Zug eingekreist und die in dem Polygon befindlichen Pixel ausgezählt wurden. Über die tatsächlich abphotographierte Fläche und die bekannte Anzahl von Kamerapixeln in der Höhe und Breite kann die rechnerische Größe eines Bildpixels und dadurch die Größe der Kontaktfläche innerhalb des Polygon-Zuges errechnet werden. 
Die nachfolgende Formel fasst die einzelnen Rechenschritte zusammen:

$K F=\left[\frac{\left[\frac{H_{p x} * B_{c m}}{B_{p x}}\right] * B_{c m}}{n_{p x \text { Kamera }}}\right] * n_{p x \text { Polygon }}$

KF: Kontaktfläche $\left[\mathrm{cm}^{2}\right]$

$\mathrm{H}_{\mathrm{px}}$ : Höhe in Pixel

$\mathrm{B}_{\mathrm{cm}}: \quad$ Breite in Zentimeter $[\mathrm{cm}]$

$\mathrm{B}_{\mathrm{px}}: \quad$ Breite in Pixel $[\mathrm{N}]$

$\mathrm{n}_{\mathrm{pxKamera}}$ Pixelanzahl der Kamera [N]

$\mathrm{n}_{\mathrm{pxP} P \text { lygon: }}$ Pixelanzahl im Polygonzug [N]

\section{Formel 3: Berechnung der Reifenkontaktfläche}

Die Anwendung dieser Methode geht von der quadratischen Form eines Pixels aus und setzt deren homogene Verteilung im digitalen Bild voraus. Dieses war bei dem verwendeten Kameramodell PowerShot A630 von Canon (Tokio, Japan) gegeben.

\section{Spurtiefen}

Die Spurtiefen wurden über den gesamten Projektzeitraum mit einer Wasserwaage und einem Gliedermaßstab erfasst: Die Wasserwaage wurde je Fahrspur an drei repräsentativen Stellen quer über die Fahrspur gelegt und die Entfernung von der unteren Kante der Wasserwaage bis in den Grund des Stollenabdrucks in der Reifenmitte gemessen. Diese Methode erwies sich trotz ihrer Schlichtheit als praktikabel, genau und transparent.

\subsection{Datenverarbeitung und statistische Auswertung}

Die Datenerfassung der einzelnen Messmethoden erfolgte wie oben beschrieben. Eine erste Datenaufbereitung fand in Microsoft Excel (Version 2003, Servicepack 2) statt. Für die weitere statistische Verrechnung der Daten wurde das Programm SPSS (Version 17.0) genutzt. Die Datensätze wurden auf 
Normalverteilung geprüft und mittels ein- oder mehrfaktorieller Varianzanalyse ANOVA untersucht. Die Post-hoc Prüfung erfolgte mithilfe der geringsten statistischen Signifikanz (LSD). Statistische Signifikanzen sind i.d.R. in den Grafiken nicht einzeln gekennzeichnet. Für Datensätze, die statistisch verrechnet wurden, ist die mittlere Grenzdifferenz der dargestellten Daten genannt. Im Anhang finden sich detaillierte Einzeldaten in denen die Grenzdifferenzen separat ausgewiesen sind.

Die Darstellung der Ergebnisse erfolgt häufig in Boxplot-Diagrammen. Sie stellen die Lage der Quartile (25\%-, 50\%- und 75\%-Perzentil) sowie die Ausreißer einer Variablen grafisch dar.

Aufgrund des hohen Stichprobenumfangs und der damit einhergehenden hohen Anzahl an Freiheitsgraden wurden tendenziell auch kleinere Unterschiede als signifikant ausgewiesen. Dieses wurde bei der Interpretation entsprechend berücksichtigt.

Die Berechnung der Korrelationen zwischen horizontalen und vertikalen Messwerten erfolgte nach Pearson. Der Koeffizient kann Werte zwischen -1 und +1 annehmen und so einen perfekten positiven linearen bzw. perfekten negativen linearen Zusammenhang zwischen zwei Messgrößen darstellen. Ein Wert von 0 gibt demnach den perfekten nicht-linearen Zusammenhang zwischen zwei Messgrößen wieder (BRosius 2004).

Der Ruhedruckkoeffizient $\mathrm{K}_{0}$ beschreibt nach HARTGE \& HORN (1999) die Beziehung zwischen vertikaler und horizontaler Hauptspannung und drückt sich wie folgt aus:

$$
\begin{array}{ll}
\sigma_{2}=\sigma_{3}=K_{0} * \sigma_{1} \\
\sigma_{1}: & \text { vertikale Hauptspannung } \\
\sigma_{2}, \sigma_{3}: & \text { horizontale Hauptspannungen } \\
\mathrm{K}_{0}: & \text { Ruhedruckkoeffizient }
\end{array}
$$

Formel 4: Beziehung zwischen Hauptspannungen und Ruhedruckkoeffizienten 
Daraus ergibt sich die Berechnung des Ruhedruckkoeffizienten als Quotient aus einer der horizontalen Hauptspannungen (erfasst durch den horizontalen Eindringwiderstand) und der vertikalen Hauptspannung (erfasst mit dem Vertikalpenetrometer):

$K_{0}=\frac{\sigma_{2 \_o d e r \_3}}{\sigma_{1}}$

Formel 5: Formel zur direkten Berechnung des Ruhedruckkoeffizienten $\mathrm{K}_{0}$ 


\section{Ergebnisse}

Nachfolgend werden die wichtigsten Ergebnisse der durchgeführten Untersuchungen dargestellt. Zur Gewährleistung der Übersichtlichtkeit der Ergebnisse finden sich die in den grafischen Darstellungen aufgezeigten Ergebnisse zusätzlich in numerischer Form im Anhang, ebenso die statistischen Kenngrößen. Hierauf wird i.d.R. im Text verwiesen.

Insgesamt erwiesen sich die homogeneren, weniger sandigen und steinfreien Böden der nordrhein-westfälischen Versuchsflächen als sensitiver gegenüber der Methode der Erfassung des Eindringwiderstandes (EW). Darüber hinaus boten die Flächen auf dem Versuchsgut Merklingsen den Vorteil, dass sie, mit Ausnahme der Bodenbearbeitung, synchron bewirtschaftet wurden. Ein besonderer Fokus bei der Darstellung der Ergebnisse wird nachfolgend auf diese Flächen gerichtet sein.

\section{1. Äußere Bedingungen der Versuchsdurchführung}

Während der Versuchsdurchführung wurden die Daten von Temperatur und Niederschlag an jedem Versuchsstandort erfasst. Eine tabellarische Darstellung der monatlichen Einzeldaten findet sich in Tabelle A 1.

Die gravimetrischen Wassergehalte der einzelnen Bodenschichten sind in Abbildung 11 zusammengefasst. In Abhängigkeit von der untersuchten Fläche, des kalendarischen Termins und der Tiefe der Probennahme wiesen die Werte teilweise deutliche Unterschiede auf und lagen in einem breiten Bereich zwischen 7,6\% und 24,2\%. Die Bodenwassergehalte der sandigen Lehme der schleswig-holsteinischen Versuchsflächen betrugen durchschnittlich 15,3\%, jene der schluffreichen nordrhein-westfälischen Flächen lagen bei 21,8\%. Die Fläche $\mathrm{SH}_{\text {konv }}$ war mit durchschnittlich $14,1 \%$ die trockenste unter den Versuchsflächen, gefolgt von $\mathrm{SH}_{\text {kons }}$ mit 16,5\%. Die Bodenwassergehalte auf den nordrhein-westfälischen Versuchsflächen lagen bei 22,6\% (NRW kons) bzw. $21,1 \%\left(\mathrm{NRW}_{\text {konv }}\right)$. 
In $40 \mathrm{~cm}$ Bodentiefe wurden mit 19,2\% die feuchtesten Verhältnisse vorgefunden, vergleichbar mit dem Bereich $20 \mathrm{~cm}$ (19,0\%) An der Bodenoberfläche wurden geringfügig niedrigere Wassergehalte (18,3\%) gemessen. In Bezug auf die Bodenbewirtschaftung wiesen die konservierend bewirtschafteten Flächen mit 19,8\% die höheren Feuchtegehalte gegenüber den konventionell bearbeiteten Flächen $(17,9 \%)$, wobei die Unterschiede in oberflächennahen Bereichen mit 3,6 Prozentpunkten am deutlichsten hervortraten.

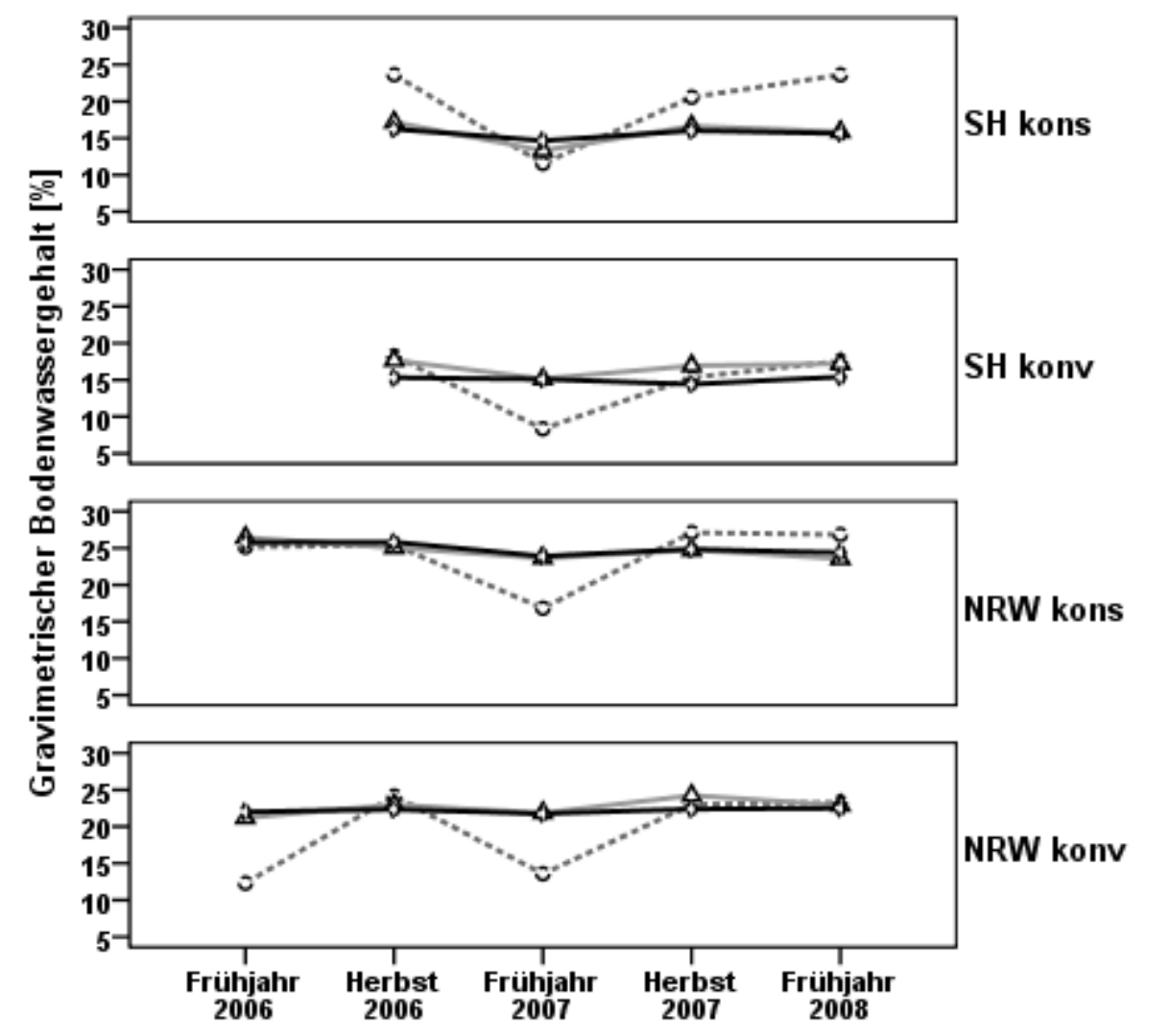

Abbildung 11: Gravimetrische Bodenwassergehalte [\%] der Versuchsflächen zu den Untersuchungsterminen in verschiedenen Bodentiefen $\left(n=2074, \mathrm{GD}_{5 \%} \approx 4,3 \%, \bar{x}\right.$ über 2 Zeitpunkte, 4 Messgruben, 5 Wdh.).

Die höchsten durchschnittlichen Bodenwassergehalte wurden im Frühjahr 2006 gemessen $(21,0 \%)$, wobei in diesen Werten aufgrund der Versuchsdurchführung nur Daten der nordrhein-westfälischen Versuchsflächen berücksichtigt sind. Die Durchschnittswerte des Herbstes 2006 (19,8\%), Herbstes 2007 $(19,4 \%)$ sowie des Frühjahres 2008 (19,1\%) lagen geringfügig unter diesem Wert. 
Augenfällig sind die Messwerte des trockenen Frühjahrs 2007, die sich mit 15,8\% (alle Messtiefen) deutlich von jenen der anderen Messzeitpunkte unterscheiden: Im Bereich der Bodenoberfläche lag der Bodenwassergehalt an diesem Termin nur bei durchschnittlich 11,7\%. In $20 \mathrm{~cm}$ betrug er $17,3 \%$ und in $40 \mathrm{~cm}$ Tiefe 18,5\%. Die gemittelten Werte der anderen vier kalendarischen Termine lagen im Bereich der Bodenoberfläche $+73 \%$ über dem des Frühjahres 2007. Mit zunehmender Bodentiefe nahm der Einfluss der Trockenheit ab und die Werte waren um $+12,6 \%$ in $20 \mathrm{~cm}$ Tiefe bzw. $+4,9 \%$ in $40 \mathrm{~cm}$ erhöht.

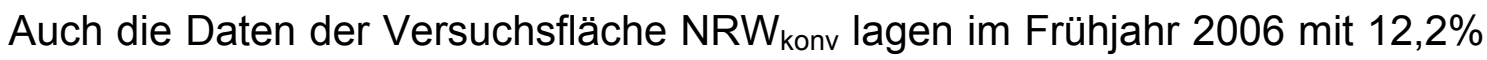
auf einem ähnlich niedrigen Niveau, da diese Fläche vor Versuchsbeginn wendend gelockert worden war und der Oberboden bei sonniger Witterung zügig abtrocknete.

Nach ZINK ET AL. (2009) lagen die Bodenwassergehalte der nordrheinwestfälischen Versuchsflächen nahe der Feldkapazität oder darüber (vgl. Tabelle A 2 und Tabelle A 3). Die durchschnittliche Bodenfeuchte betrug in $20 \mathrm{~cm}$ Tiefe durchschnittlich $96 \%$, in $40 \mathrm{~cm}$ lag sie bei $100 \%$. Für die schleswigholsteinischen Flächen ermittelte Zink Werte von 85\% (20 cm Tiefe) bzw. 90\% in $40 \mathrm{~cm}$ Tiefe (vgl. Tabelle A 4 und Tabelle A 5).

\subsection{Kontaktflächen und Kontaktflächendrücke}

Die Ergebnisse der gemessenen Kontaktflächen und der daraus errechneten Kontaktflächendrücke wiesen eine breite Streuung der Werte sowohl in Bezug auf die Versuchsflächen als auch über den Untersuchungszeitraum auf. Zum einen unterschieden sich die Messtermine stark bezüglich der vorherrschenden Bodenverhältnisse: Bodenbearbeitungsmaßnahmen unmittelbar vor einer Befahrung, der auf der Fläche zum Messzeitpunkt gegebene Pflanzenbewuchs und die Bodenwassergehalte hatten einen starken Einfluss auf die Messwerte. So setzte sich die Kontaktfläche bei abgesetztem und durchwurzeltem Boden (Bsp. SH im Herbst 2006) aus einzelnen Teilflächen zusammen, die lediglich der Aufstandsfläche der Reifenstollen entsprachen. Am selben Standort konnten im Frühjahr 2007 auf gepflügtem Acker deutlich höhere Kontaktflächen 
ermittelt werden, da sich der Reifen tiefer in den Boden eindrückte und die Radlast auch im Bereich des Stollenzwischenraumes abgestützt wurde.

Die über den Projektzeitraum ermittelten Ergebnisse der Kontaktflächenmessungen sind in Tabelle 4 dargestellt, eine Zusammenfassung der Kontaktflächendrücke findet sich in Tabelle A 10. 
Tabelle 4: Mittlere Kontaktflächen $\left[\mathrm{cm}^{2}\right]$ der Belastungsvarianten nach Fläche und Termin für Michelin MegaXBib ${ }^{\circledR}$ 650/75 R32 (MachXBib 650/75 R38 für Frühjahr 2006, x über 6 Varianten)

\begin{tabular}{|c|c|c|c|c|c|}
\hline Variante & Termin & $\mathrm{SH}_{\text {kons }}$ & $\mathrm{SH}_{\text {konv }}$ & $\mathrm{NRW}_{\text {kons }}$ & $\mathrm{NRW}_{\text {konv }}$ \\
\hline \multirow[t]{5}{*}{ 3,3Mg@50kPa } & Frühjahr 2006 & k.A. & k.A. & 5.941 & 4.006 \\
\hline & Herbst 2006 & 5.836 & 1.266 & 4.573 & 2.948 \\
\hline & Frühjahr 2007 & 1.276 & 3.261 & 1.540 & k.A. \\
\hline & Herbst 2007 & 4.827 & 3.185 & 3.112 & 1.454 \\
\hline & Frühjahr 2008 & 4.166 & 1.269 & 4.372 & 4.316 \\
\hline \multirow[t]{5}{*}{ 3,3Mg@160kPa } & Frühjahr 2006 & k.A. & k.A. & 4.084 & 2.696 \\
\hline & Herbst 2006 & 2.830 & 912 & 4.283 & 1.862 \\
\hline & Frühjahr 2007 & 719 & 3.021 & 1.204 & 977 \\
\hline & Herbst 2007 & 2.780 & 2.623 & 2.316 & 1.013 \\
\hline & Frühjahr 2008 & 3.241 & 805 & 3.141 & 3.148 \\
\hline \multirow[t]{5}{*}{ 6,3Mg@160kPa } & Frühjahr 2006 & k.A. & k.A. & 6.592 & 4.129 \\
\hline & Herbst 2006 & 5.152 & 1.326 & 3.664 & 4.358 \\
\hline & Frühjahr 2007 & 1.144 & 4.288 & 2.958 & 1.600 \\
\hline & Herbst 2007 & 4.780 & 4.876 & 3.600 & 3.531 \\
\hline & Frühjahr 2008 & 4.104 & 3.949 & 4.507 & 4.636 \\
\hline \multirow[t]{5}{*}{ 6,3Mg@250kPa } & Frühjahr 2006 & k.A. & k.A. & 5.272 & 3.914 \\
\hline & Herbst 2006 & 3.387 & 1.322 & 2.682 & 2.326 \\
\hline & Frühjahr 2007 & 1.084 & 3.251 & 1.175 & 1.368 \\
\hline & Herbst 2007 & 3.989 & 3.172 & 3.080 & 3.545 \\
\hline & Frühjahr 2008 & 3.852 & 3.660 & 4.233 & 3.620 \\
\hline \multirow[t]{5}{*}{ 7,5Mg@250kPa } & Frühjahr 2006 & k.A. & k.A. & k.A. & k.A. \\
\hline & Herbst 2006 & 4.477 & 1.405 & 4.699 & 4.218 \\
\hline & Frühjahr 2007 & 1.243 & 4.026 & 2.750 & 1.653 \\
\hline & Herbst 2007 & 4.715 & 5.997 & 3.323 & 3.327 \\
\hline & Frühjahr 2008 & 3.858 & 3.251 & 4.430 & 3.623 \\
\hline \multirow[t]{5}{*}{ 7,5Mg@350kPa } & Frühjahr 2006 & k.A. & k.A. & k.A. & k.A. \\
\hline & Herbst 2006 & 3.726 & 843 & 4.246 & 3.939 \\
\hline & Frühjahr 2007 & 1.124 & 3.660 & 2.560 & 1.536 \\
\hline & Herbst 2007 & 4.010 & 3.053 & k.A. & 3.699 \\
\hline & Frühjahr 2008 & 3.651 & 3.170 & 4.241 & 3.505 \\
\hline
\end{tabular}

Trotz der unterschiedlichen Verhältnisse zeigen die Messungen klare Ergebnisse: Die Kontaktflächen auf den schluffreichen nordrhein-westfälischen Versuchsflächen lagen mit einer Kontaktfläche von durchschnittlich $3.325 \mathrm{~cm}^{2}$ 
um durchschnittlich $+8,1 \%$ über denen der sandreicheren schleswigholsteinischen Flächen $\left(3.074 \mathrm{~cm}^{2}\right)$.

Konservierend bewirtschaftete Flächen wiesen mit durchschnittlich $3.501 \mathrm{~cm}^{2}$ eine um $+20,2 \%$ größere Kontaktfläche auf als Flächen bei konventioneller Bodenbewirtschaftung $\left(2.913 \mathrm{~cm}^{2}\right)$. Eine Stetigkeit war hierbei jedoch nicht gegeben und in Anhängigkeit vom zeitlichen Abstand der Kontaktflächenmessung zum Zeitpunkt der letztmaligen Bodenbearbeitungsmaßnahme konnten auf den konventionell bewirtschafteten Flächen z. T. deutlich größere Aufstandsflächen ermittelt werden.

Die Versuchfaktoren Radlast und Reifeninnendruck wiesen einen charakteristischen Einfluss auf die Größe der Kontaktflächen und damit auf die Kontaktflächendrücke auf. So steigt bei einer Zunahme der Radlast von 3,3 Mg auf $6,3 \mathrm{Mg}$ bei konstantem Reifeninnendruck von $160 \mathrm{kPa}$ die durchschnittliche Kontaktfläche um $1.530 \mathrm{~cm}^{2}$ oder $+66,1 \%$ an. Bei $250 \mathrm{kPa}$ Reifeninnendruck beträgt die durch die Erhöhung der Radlast von $6,3 \mathrm{Mg}$ auf 7,5 Mg bedingte relative Zunahme der Kontaktfläche noch $+16,7 \%$.

Die Absenkung des Reifeninnendruckes führte unabhängig von den Verhältnissen stets zu einer Zunahme der Kontaktflächen und einer Abnahme der Kontaktflächendrücke. Bei einer Radlast von 3,3 Mg und einer Absenkung des Reifeninnendruckes von $160 \mathrm{kPa}$ auf $50 \mathrm{kPa}$ betrug die Zunahme der Kontaktfläche durchschnittlich $+45,8 \%$. Bei 6,3 Mg Radlast und der Absenkung von $250 \mathrm{kPa}$ auf $160 \mathrm{kPa}$ waren es noch $+26,0 \%$ und bei $7,5 \mathrm{Mg}$ und einer Anpassung von $350 \mathrm{kPa}$ auf $250 \mathrm{kPa}$ war noch eine Zunahme der Kontaktfläche von $+13,8 \%$ zu beobachten.

\subsection{Spurtiefen}

Die Tiefe der Fahrspuren nach einer Befahrung ist das augenfälligste Merkmal einer Bodenveränderung durch mechanische Belastung. Die Bodenart, der Bearbeitungszustand des Bodens zum Zeitpunkt der Befahrung, der Bodenwassergehalt und die Fahrwerksparameter Radlast und Reifeninnendruck nehmen entscheidenden Einfluss auf die Ausprägung der Fahrspuren. 
Analog zu den Messungen der Kontaktflächen und Kontaktflächendrücke wiesen auch die Spurtiefenmessungen eine breite Streuung der Werte auf. Die zusammengefassten Ergebnisse sind in Abbildung 12 dargestellt.

Variante

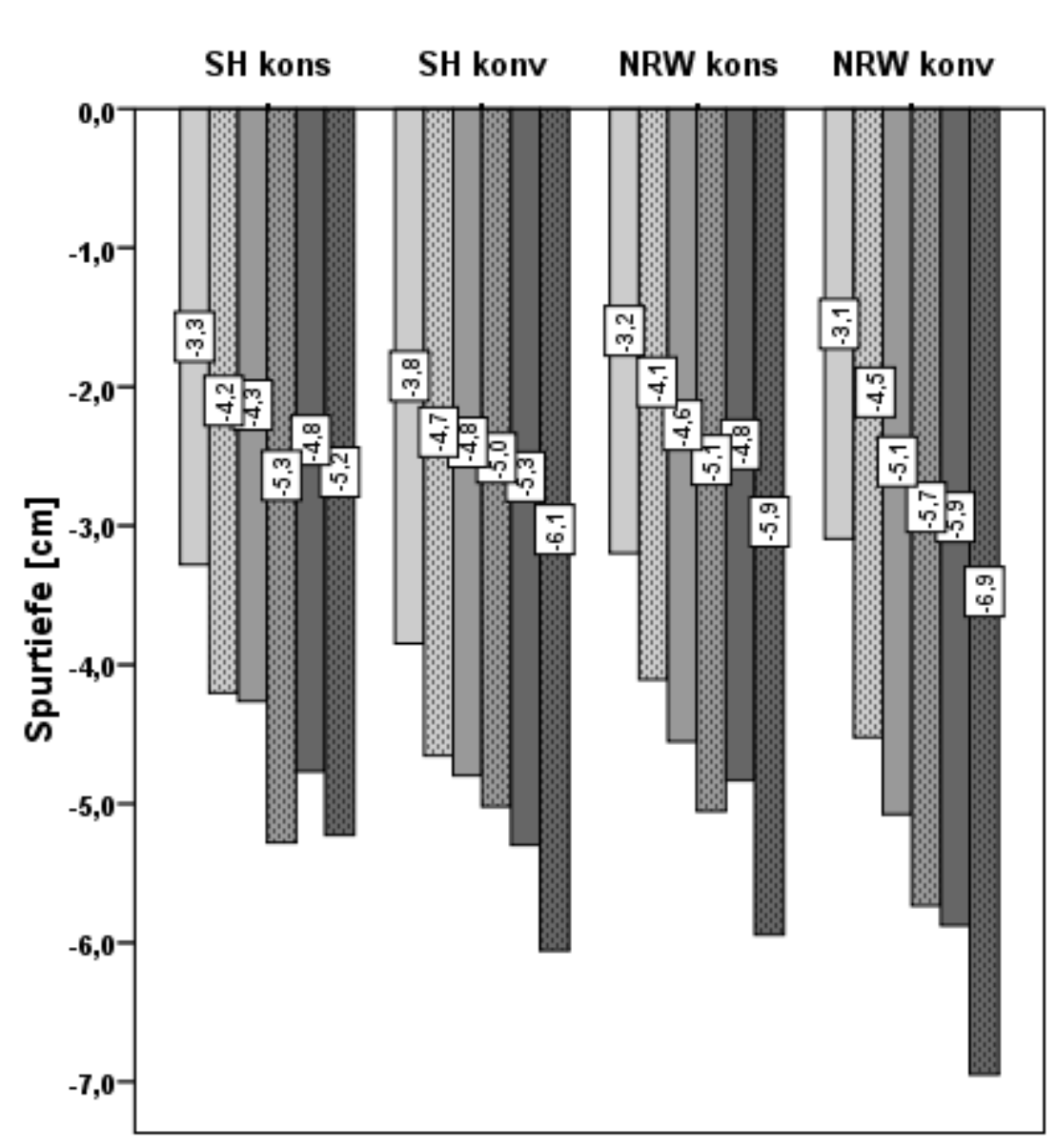

$\square 3,3 \mathrm{Mg} @ 50 \mathrm{kPa}$ 3,3 Mg@ $160 \mathrm{kPa}$ $\square 6,3 \mathrm{Mg} @ 160 \mathrm{kPa}$ 6,3 Mg@ $250 \mathrm{kPa}$

$\square 7,5 \mathrm{Mg} @ 250 \mathrm{kPa}$

7,5 Mg@350 kPa

Abbildung 12: Spurtiefen der Befahrungen nach Fläche und Belastungsvariante ( $n=1.980, G_{5 \%} \approx 0,42 \mathrm{~cm}, \bar{x}$ über 5 Termine, 2 Blöcke, 15 Wdh.).

Im Mittel über den Untersuchungszeitraum wiesen die Versuchsstandorte NRW mit $-5,0 \mathrm{~cm}$ Spurtiefe und $\mathrm{SH}$ mit $-4,7 \mathrm{~cm}$ Spurtiefe (SH) signifikante Unterschiede zueinander auf. Der Einfluss einer reduzierten Bodenbearbeitung auf die Spurtiefen ist an beiden Standorten vergleichbar: am Standort NRW beträgt die Spurtiefe bei wendender Bodenbearbeitung $-5,2 \mathrm{~cm}$ während sie bei konservierender Bewirtschaftung bei $-4,7 \mathrm{~cm}$ liegt. In $\mathrm{SH}$ findet sich ein identischer Unterschied auf einem um 0,2 cm höheren Niveau. Die tiefsten Fahrspuren waren auf den konventionellen Flächen $\operatorname{NRW}_{\text {konv }}(-5,2 \mathrm{~cm})$ und 
$\mathrm{SH}_{\text {konv }}(-5,0 \mathrm{~cm}) \mathrm{zu}$ finden. Auf den konservierend bewirtschafteten Flächen $\mathrm{NRW}_{\text {kons }}$ und $\mathrm{SH}_{\text {kons }}$ lagen sie bei $-4,7 \mathrm{~cm}$ bzw. $-4,5 \mathrm{~cm}$.

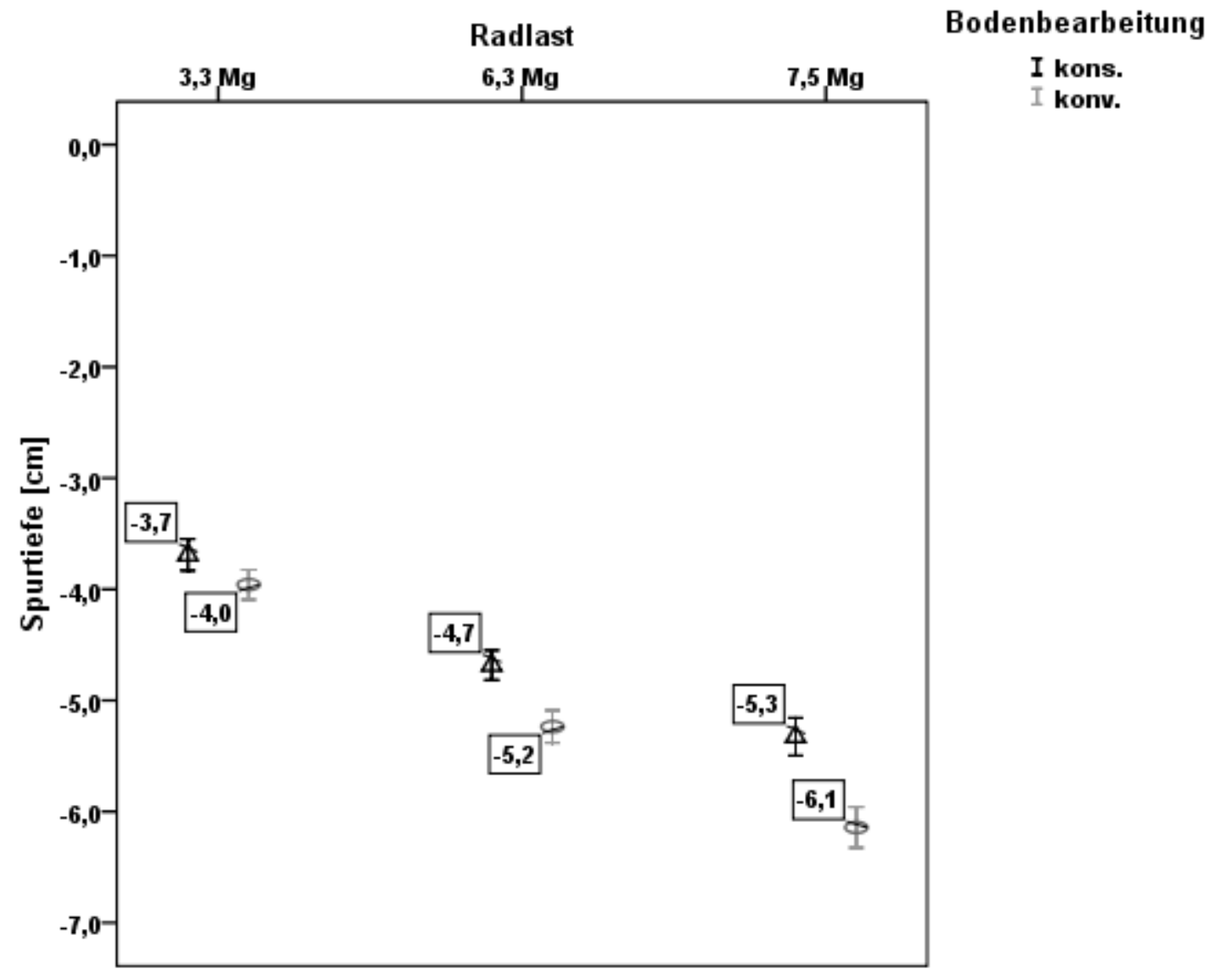

Abbildung 13: Mittelwerte der gemessenen Spurtiefen ( \pm Standardfehler) bei verschiedenen Radlasten in Abhängigkeit des angewendeten Bodenbearbeitungssystems ( $n=1.980, \mathrm{GD}_{5 \%} \approx 0,21 \mathrm{~cm}, \overline{\mathrm{x}}$ über 2 Zeitpunkte, 2 Reifeninnendrücke, 2 Blöcke, $15 \mathrm{Wdh}$.).

In Abhängigkeit von der Radlast lagen die Spurtiefen bei $-3,8 \mathrm{~cm}$ (3,3 Mg), $-5,0 \mathrm{~cm}(6,3 \mathrm{Mg})$ und $-5,7 \mathrm{~cm}(7,5 \mathrm{Mg})$ und wurden von diesem Faktor signifikant beeinflusst. Die Erhöhung der Radlast von $3,3 \mathrm{Mg}$ auf $6,3 \mathrm{Mg}$ bei jeweils 160 kPa Reifeninnendruck führte zu einer Zunahme der Spurtiefen von $-4,4 \mathrm{~cm}$ auf $-4,7 \mathrm{~cm}$. Hierbei betrugen die absoluten Änderungen bezogen auf den jeweiligen Standort 0,1 cm (SH) bzw. 0,5 cm (NRW). Wurde die Radlast bei $250 \mathrm{kPa}$ RID von 6,3 Mg auf 7,5 Mg erhöht, war keine Zunahme der Spurtiefe mehr zu beobachten. Sie betrug an beiden Standorten $-5,2 \mathrm{~cm}$. 


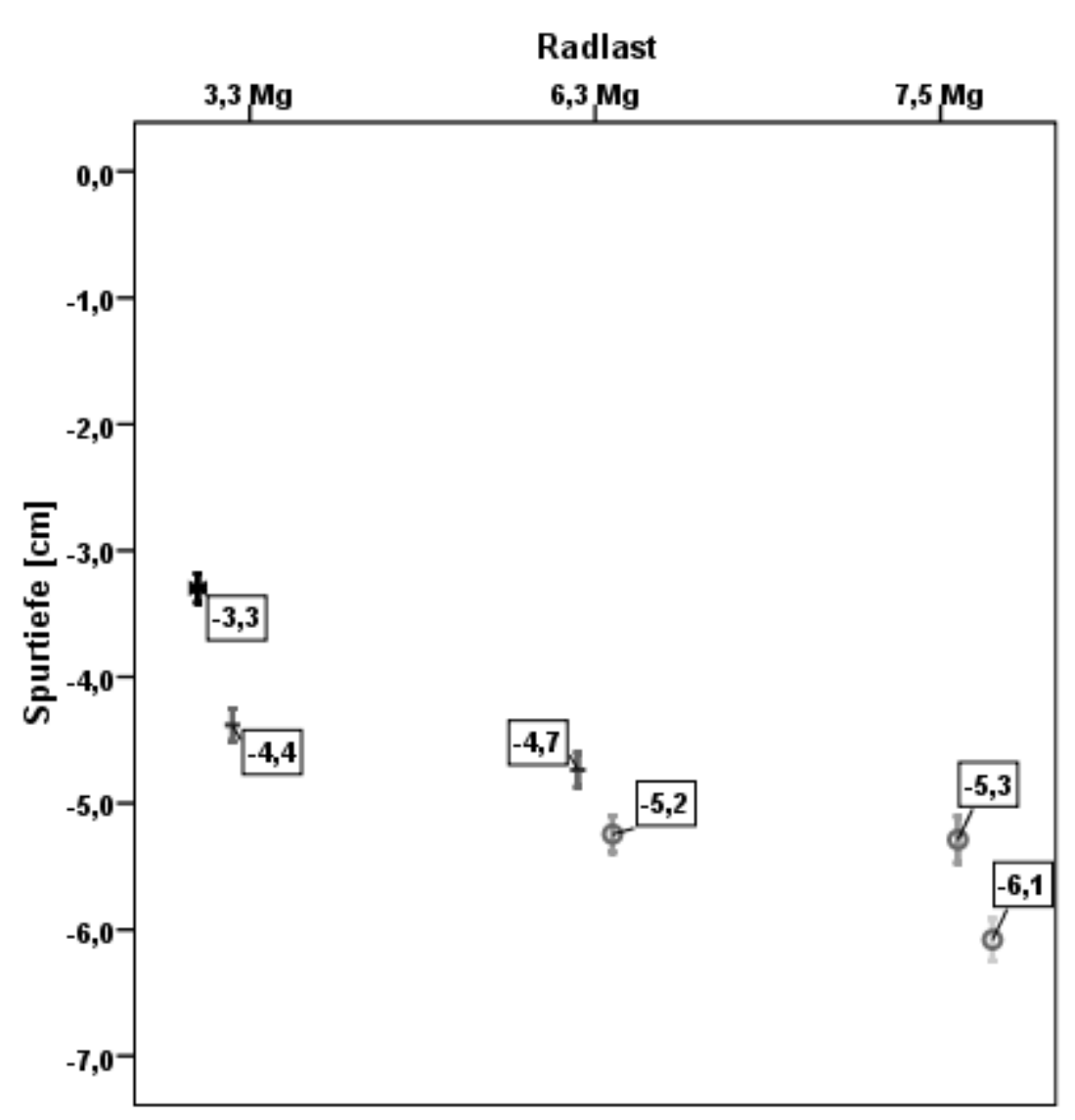

Reifeninnendruck

I $50 \mathrm{kPa}$

$1160 \mathrm{kPa}$

$I 250 \mathrm{kPa}$

$350 \mathrm{kPa}$

Abbildung 14: Mittelwerte der gemessenen Spurtiefen ( \pm Standardfehler) bei verschiedenen Radlasten in Abhängigkeit des eingestellten Reifeninnendruckes ( $n=1.980, G D_{5 \%} \approx 0,21 \mathrm{~cm}, \bar{x}$ über 2 Zeitpunkte, 2 Reifeninnendrücke, 2 Blöcke, 15 Wdh.).

Auch der Faktor Bodenbearbeitung weist bei allen Radlasten einen signifikanten Einfluss auf die gemessenen Spurtiefen auf, wobei die konservierend bewirtschafteten Flächen mit durchschnittlich $-4,6 \mathrm{~cm}$ geringere Spurtiefen ausweisen als die konventionell bewirtschafteten $(-5,1 \mathrm{~cm})$. Mit steigenden Radlasten ist auch ein steigender Einfluss des Bodenbearbeitungssystems zu beobachten (vgl. Abbildung 13): Für 3,3 Mg beträgt die Spurtiefe auf den konservierend bewirtschafteten Flächen $-3,7 \mathrm{~cm}$ gegenüber $-4,0 \mathrm{~cm}$ auf den Flächen unter Pflug. Bei 6,3 Mg erhöht sich diese Differenz auf 0,5 cm und bei $7,5 \mathrm{Mg}$ beträgt sie bereits $0,8 \mathrm{~cm}$. Dieses konnte unabhängig vom Versuchsstandort beobachtet werden, allerdings zeigen die Daten aus NRW mit Unterschieden von 0,2 cm (bei 3,3 Mg), 0,6 cm (bei 6,3 Mg) und 0,9 cm (bei 7,5 Mg) einen klareren Einfluss des Bodenbearbeitungssystems bei steigenden 
Radlasten als jene der schleswig-holsteinischen Versuchsflächen $(0,5 \mathrm{~cm}$, $0,4 \mathrm{~cm}, 0,7 \mathrm{~cm}$ respektive).

Eine Reduzierung des Reifeninnendruckes führt unabhängig von Bodenbearbeitungssystem und Radlast stets zu einer Abnahme der Spurtiefe - im Durchschnitt um 0,9 cm (vgl. Abbildung 14). Bei einer Radlast von 3,3 Mg zeigt eine Absenkung des Reifeninnendruckes von $160 \mathrm{kPa}$ auf $50 \mathrm{kPa}$ mit einer Abnahme der Spurtiefe um durchschnittlich $1,1 \mathrm{~cm}$ den stärksten Effekt, während die Absenkung des Reifeninnendruckes von $250 \mathrm{kPa}$ auf $160 \mathrm{kPa}$ bei einer Radlast von $6,3 \mathrm{Mg}$ die Spurtiefe um durchschnittlich $0,5 \mathrm{~cm}$ reduziert. Für beide Radlastvarianten zeigen sich auf den nordrhein-westfälischen Versuchsflächen die deutlicheren Effekte auch in Bezug auf das angewendete Bodenbearbeitungssystem.

\subsection{Vertikaler Eindringwiderstand}

Die Erfassung der vertikalen Eindringwiderstände vor und nach der Befahrung eines jeden Untersuchungszeitpunktes führte zu einem großen Datenaufkommen, da pro Messung 81 Einzeldaten $(0-80 \mathrm{~cm}$ Bodentiefe) erfasst wurden. Um die Auswertbarkeit und Bezugstellung zu den TASIs-Daten herstellen zu können, bedurfte es einer Datenreduktion. Abbildung 15 veranschaulicht das Vorgehen der Reduktion auf vier Messtiefenbereiche wie in Abschnitt 3.4 beschrieben, indem die klassische Darstellung des vEW als Funktion über die Messtiefe in Form eines Liniendiagramms (rechte Seite) der Darstellung der gemittelten Werte der entsprechenden Messtiefenbereiche als Boxplot-Diagramm (linke Seite) gegenübergestellt wurde.

Um einen Überblick über den Datensatz zu gewinnen, werden zunächst die vEW vor Versuchsbeginn betrachtet und die Ausgangssituation analysiert. Anschließend wird der Datensatz auf Effekte der Parameter Standort und Bodenbearbeitung sowie der weiteren Faktoren Termin, Messtiefenbereich und Messzeitpunkt untersucht. Anschließend wird der Einfluss der mechanischen Belastung sowie der Fahrwerksparameter Radlast und Reifeninnendruck betrachtet. 


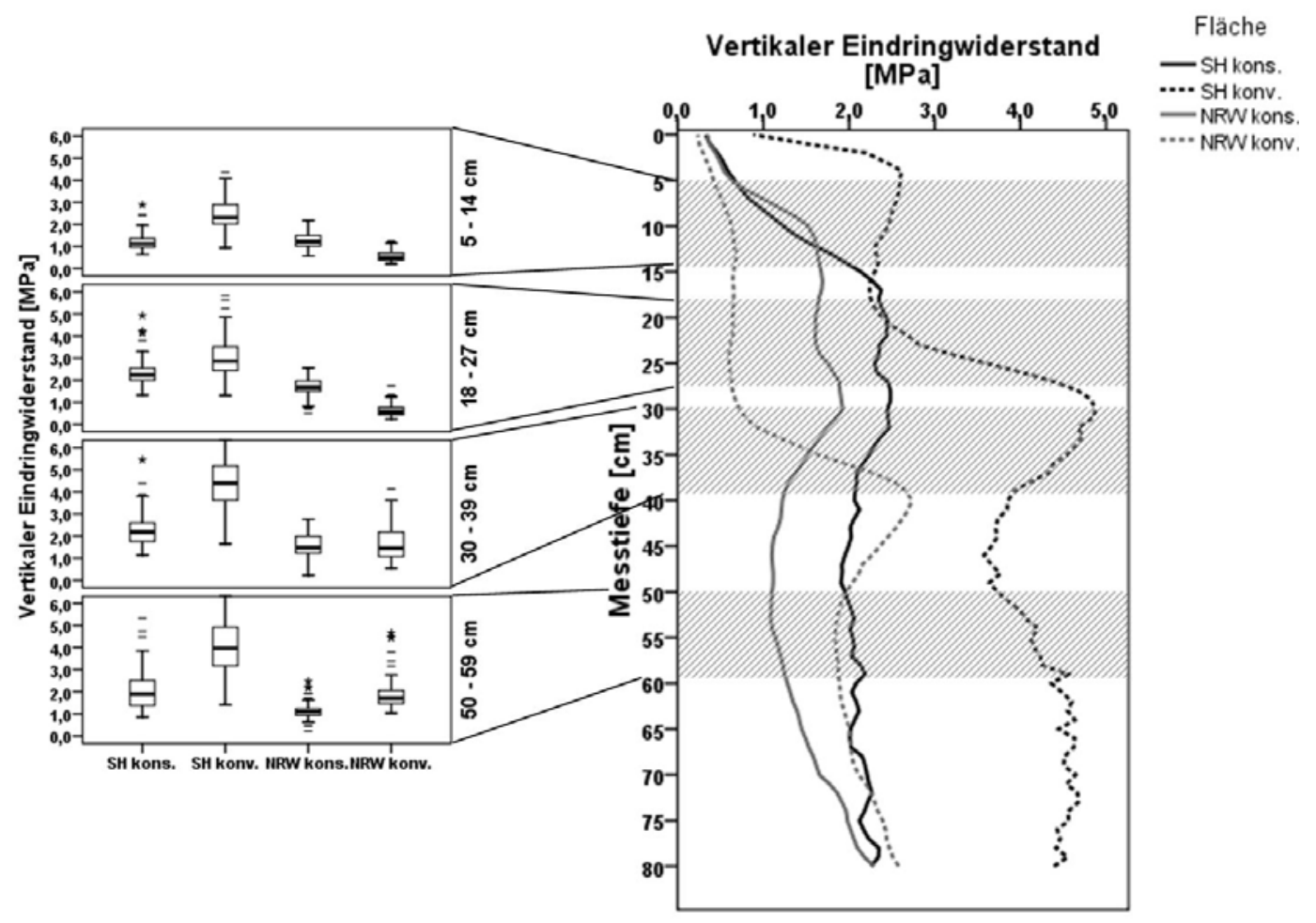

Abbildung 15: Vertikale Eindringwiderstände der Versuchsflächen vor Versuchsbeginn 2006 in den Darstellungsweisen Boxplot- (links) und Liniendiagramm (rechts) $\left(\mathrm{GD}_{5 \%} \approx 0,1 \mathrm{MPa}\right)$.

\subsubsection{Ausprägung des vertikalen Eindringwiderstandes auf den einzelnen Versuchsflächen vor Versuchsbeginn}

Die Darstellungen in Abbildung 15 lassen die auf den jeweiligen Flächen praktizierte langjährige Bodenbewirtschaftung erkennen. Sowohl auf $\mathrm{NRW}_{\text {konv }}$ als auch auf $\mathrm{SH}_{\text {konv }}$ finden sich in Tiefe $35-45 \mathrm{~cm}$ bzw. $30-35 \mathrm{~cm}$ Bereiche erhöhter Eindringwiderstände von $>2,5 \mathrm{MPa}\left(\mathrm{NRW}_{\text {konv }}\right)$ bzw. $>3,5 \mathrm{MPa}$ $\left(\mathrm{SH}_{\mathrm{konv}}\right)$, wie sie bei wendender Bodenbearbeitung typischerweise vorkommen. Oberhalb dieser Sohle befindet sich der Bearbeitungshorizont. Hier ist der Boden lockerer gelagert und weist niedrigere Werte auf. Werden die Sohlen von der Messsonde durchstoßen, so können auch in den darunter liegenden Bodenschichten wiederum geringere Eindringwiderstände beobachtet werden, die allerdings nicht mehr auf das Niveau des Bearbeitungshorizontes absinken und stets über jenen der konservierend bewirtschafteten Fläche des jeweiligen Standortes in der entsprechenden Tiefe liegen. 
Auf den konservierend bewirtschafteten Flächen kann ein solch deutlich ausgeprägter Bereich erhöhter Eindringwiderstände nicht festgestellt werden: Die Werte steigen bis in eine Tiefe von $10-15 \mathrm{~cm}$ an und verlaufen dann relativ gleich bleibend bis in eine Tiefe von $30-35 \mathrm{~cm}$, bevor sie wieder leicht abfallen. Dichter gelagerte Bereiche evtl. reliktischer Pflugsohlen können auf den langjährig konservierend bewirtschafteten Versuchsflächen nicht identifiziert werden.

Zum Zeitpunkt der Nullbeprobung vor Versuchsbeginn konnten auf den Standorten signifikant unterschiedliche vEW gemessen werden. der über die Messtiefenbereiche gemittelte Wert für die nordrhein-westfälischen Versuchsflächen lag bei 1,28 $( \pm 0,17) \mathrm{MPa}$, in Schleswig-Holstein wurden deutlich höhere Werte von 2,75 $( \pm 0,87)$ MPa ermittelt. Neben dem Einfluss des Standortes war hierbei auch der Einfluss der Bodenbearbeitung deutlich erkennbar: mit durchschnittlich 2,12 $( \pm 1,21) \mathrm{MPa}$ lag der vEW bei konventioneller Bewirtschaftung signifikant über dem mittleren vEW von 1,68 $( \pm 0,35)$ MPa bei konservierender Bewirtschaftung, so dass sich auch alle Flächen untereinander jeweils signifikant voneinander unterschieden.

Bei diesen Unterschieden gilt es allerdings $\mathrm{zu}$ berücksichtigen, dass die Flächen nicht zum gleichen Zeitpunkt (NRW im Frühjahr 2006, SH im Herbst 2006) beprobt wurden und der Beprobung eine unterschiedlich intensive Bodenbearbeitung (NRW konv wendend bearbeitet, $\mathrm{NRW}_{\text {kons }}$ keine Bodenbearbeitung) unmittelbar vorausgegangen war.

Auf keiner der nordrhein-westfälischen Versuchsflächen konnten zum Zeitpunkt der Nullbeprobung signifikante Unterschiede zwischen Kontrollparzellen und solchen, die für Befahrungen vorgesehen waren, festgestellt werden (vgl. 
Tabelle A 14). Anders verhielt es sich auf den schleswig-holsteinischen Versuchsflächen: auf $\mathrm{SH}_{\text {kons }}$ wiesen die Parzellen der Varianten 6,3Mg@160kPa und 7,5Mg@350kPa vor Versuchsbeginn signifikant niedrigere vEW gegenüber der Kontrolle auf. Auf $\mathrm{SH}_{\mathrm{konv}}$ waren es die Varianten 3,3Mg@160kPa und 6,3Mg@160kPa, bei denen ein signifikant niedrigerer vEW gegenüber der Kontrolle gemessen wurde, während die in den Parzellen der Variante 7,5Mg@250kPa gemessenen Werte signifikant oberhalb der Kontrolle lagen.

\subsubsection{Effekte der Faktoren Standort, Bodenbearbeitung sowie besonderer Wetterereignisse}

In der statistischen Betrachtung der Haupteffekte Standort und Bodenbearbeitung wurden Unterschiede zwischen den einzelnen Versuchsflächen deutlich: im Mittel aller Messungen wurde am Standort NRW ein $\mathrm{VEW}$ von $1,69( \pm 0,71) \mathrm{MPa}$ gemessen, der einem Wert von $2,34( \pm 1,17) \mathrm{MPa}$ auf den schleswig-holsteinischen Versuchsflächen gegenüberstand. Diese Differenz konnte als statistisch signifikant abgesichert werden. Der Gesamtmittelwert aller Messungen lag bei 1,94 ( $\pm 0,97) \mathrm{MPa}$. Flächen mit konservierender Bodenbewirtschaftung wiesen mit $1,85( \pm 0,82) \mathrm{MPa}$ gegenüber 2,02 $( \pm 1,07) \mathrm{MPa}$ einen statistisch signifikant niedrigeren Wert gegenüber konventionell bewirtschafteten Flächen auf.

Ein Vergleich der Mittelwerte der einzelnen Flächen zeigt, dass sich $\mathrm{NRW}_{\text {kons }}$ $(1,68 \pm 0,59 \mathrm{MPa})$ und $\mathrm{NRW}_{\text {konv }}(1,71 \pm 0,78 \mathrm{MPa})$ nicht signifikant voneinander unterscheiden. Die schleswig-holsteinischen Versuchsflächen $\mathrm{SH}_{\text {kons }}$ $(2,08 \pm 1,01 \mathrm{MPa})$ und $\mathrm{SH}_{\text {konv }}(2,60 \pm 1,26 \mathrm{MPa})$ weisen hingegen signifikante Unterschiede auf - sowohl voneinander als auch jeweils gegenüber den beiden nordrhein-westfälischen Flächen.

Der Faktor Bodenbearbeitung ist bedeutend nicht nur in Bezug auf die langfristigen Effekte einer unterschiedlich intensiven Bodenlockerung - bspw. durch die Ausprägung einer Pflugsohle - sondern er nimmt auch erkennbaren Einfluss auf die Messwerte der vEW vor und nach einer Befahrung. Besonders deutlich konnte dieses an den Terminen beobachtet werden, an denen unmittelbar vor der Befahrung eine Bodenbearbeitung durchgeführt worden 
war. Dieses war bei den Messungen des Frühjahres 2006 auf $\mathrm{NRW}_{\text {konv }}$ und im Frühjahr 2007 auf $\mathrm{SH}_{\text {konv }}$ der Fall (vgl. Abbildung 16 und Abbildung 17).

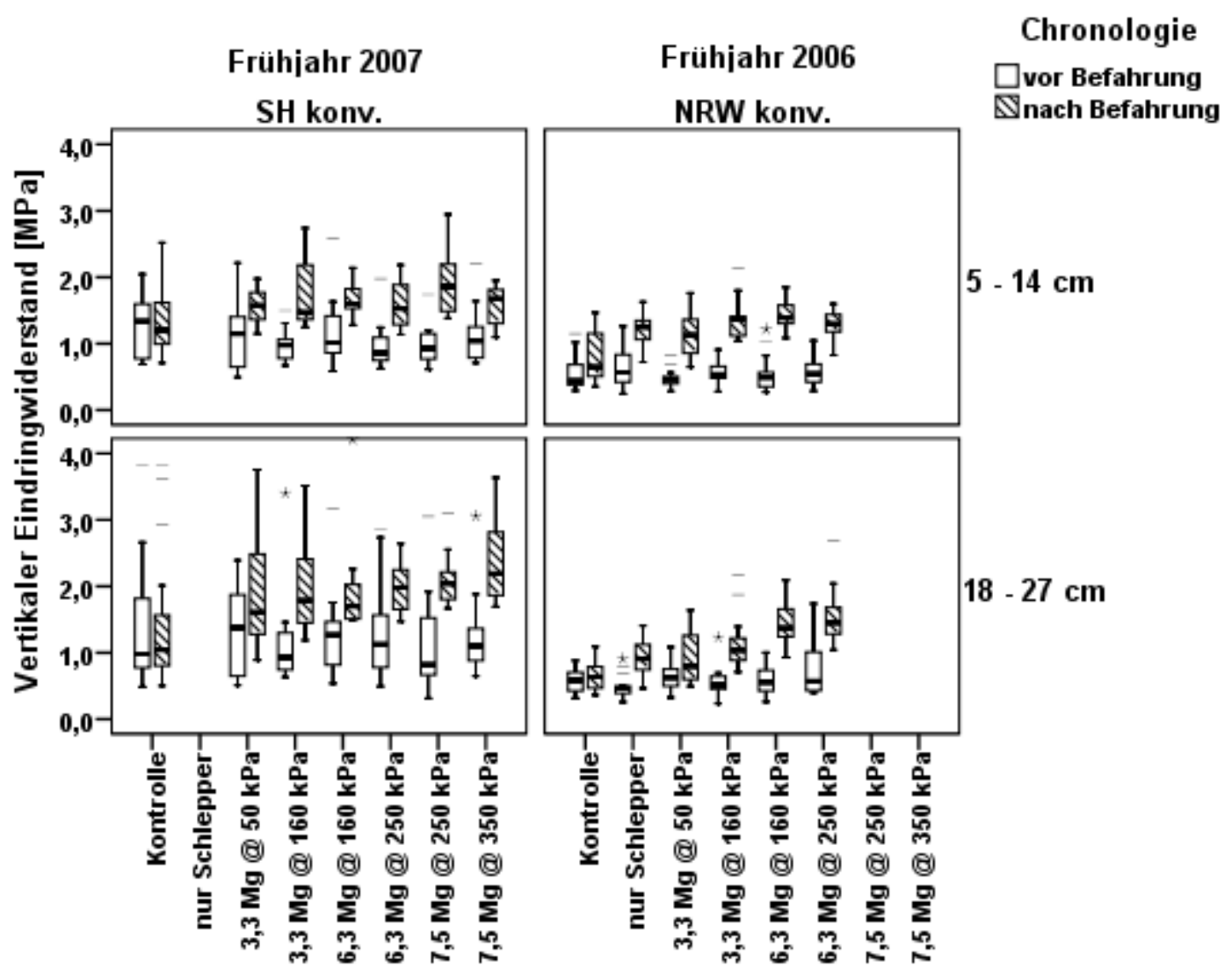

Abbildung 16: Effekte einer Befahrung auf den vertikalen Eindringwiderstand nach zuvor erfolgter wendender Bodenbearbeitung in den Messtiefenbereichen $5-14 \mathrm{~cm}$ und 18 $27 \mathrm{~cm}\left(\mathrm{n}=780, \mathrm{GD}_{5 \%} \approx 0,30 \mathrm{MPa}, \overline{\mathrm{x}}\right.$ über 2 Blöcke, $6 \mathrm{Wdh}$.).

Während sich in den beiden dargestellten Fällen die vEW der unbefahrenen Parzellen in den Tiefen bis $39 \mathrm{~cm}$ zum Zeitpunkt nach der Befahrung nur um +0,06 MPa gegenüber der Messung vor der Befahrung unterschieden, konnten in den befahrenen Parzellen vEW gemessen werden, die gegenüber dem Ausgangsniveau um durchschnittlich $+0,42 \mathrm{MPa}$ erhöht waren. Hierbei konnten in den Bereichen $5-14 \mathrm{~cm}(+0,64 \mathrm{MPa})$ und $18-27 \mathrm{~cm}(+0,68 \mathrm{MPa})$ im Mittel aller Varianten signifikant höhere $\mathrm{vEW}$ nach der Befahrung ermittelt werden (vgl. Abbildung 16). Im Messtiefenbereich $30-39 \mathrm{~cm}$ wurden uneinheitliche Änderungen im vEW gemessen, nicht i.d.R. unterhalb der Signifikanzgrenze bewegten. 
Hierbei fällt auf, dass die Zunahmen der vEW in der obersten Messtiefe unabhängig von der Intensität der mechanischen Auflast erfolgen und sich für alle Belastungsvarianten in einem Bereich zwischen $\pm 0,5 \mathrm{MPa}$ und $\pm 0,9 \mathrm{MPa}$ bewegen. Im darunter liegenden Bereich $18-27 \mathrm{~cm}$ ist erkennbar, dass mit steigenden mechanischen Auflasten auch die vEW nach der Befahrung zunehmen. Ein signifikanter Anstieg der vEW infolge einer mechanischen Belastung kann in den beiden oberen Messtiefen unabhängig vom Standort nachgewiesen werden.

Gleiches gilt für nicht-signifikante Änderungen der vEW im Bereich $30-39 \mathrm{~cm}$. Die Belastungsvariante 6,3Mg@160kPa auf $\mathrm{NRW}_{\mathrm{konv}}$ stell hierbei eine Ausnahme dar, da sie ebenfalls eine signifikante Zunahme der vEW aufweist. In der Messtiefe $30-39 \mathrm{~cm}$ konnten im Mittel der befahrenen Parzellen keine nennenswerte Änderung der Messwerte (-0,02 MPa) festgestellt werden (vgl. Abbildung 17).

Neben dem Einfluss einer unmittelbar zuvor erfolgten wendenden Bodenbearbeitung konnte innerhalb des Gesamtdatensatzes eine weitere Konstellation auffälliger Messwerte am Standort Schleswig-Holstein im Herbst 2006 beobachtet werden, als die erste VP-Messung und die anschließende Befahrung durch ein Starkregenereignis unterbrochen wurden. Die Messwerte zeigen den deutlichen Trend niedrigerer vEW nach der Befahrung (vgl. Abbildung 18 und Abbildung 19).

Von den 56 untersuchten Fläche-Variante-Messtiefe-Konstellationen wurde in 46 Fällen nach der Befahrung ein niedrigerer vEW gemessen, der in 19 Fällen als statistisch signifikant abgesichert werden konnte (vgl. Tabelle A 17). Von 14 auf $\mathrm{SH}_{\text {kons }}$ dokumentierten Fälle befanden sich 12 in den beiden oberen Messtiefenbereichen bis $27 \mathrm{~cm}$ Bodentiefe, während auf $\mathrm{SH}_{\text {konv }} 40 \%$ der statistisch signifikanten Fälle im Unterboden $(50-59 \mathrm{~cm})$ festgestellt wurden. In den Bereichen $30-39 \mathrm{~cm}$ und $18-27 \mathrm{~cm}$ wies jeweils die Belastungsvariante 7,5Mg@250kPa nach der Befahrung statistisch signifikant niedrigere vEW auf, in 18 - 27 cm war es zusätzlich die Variante 7,5Mg@350kPa. 


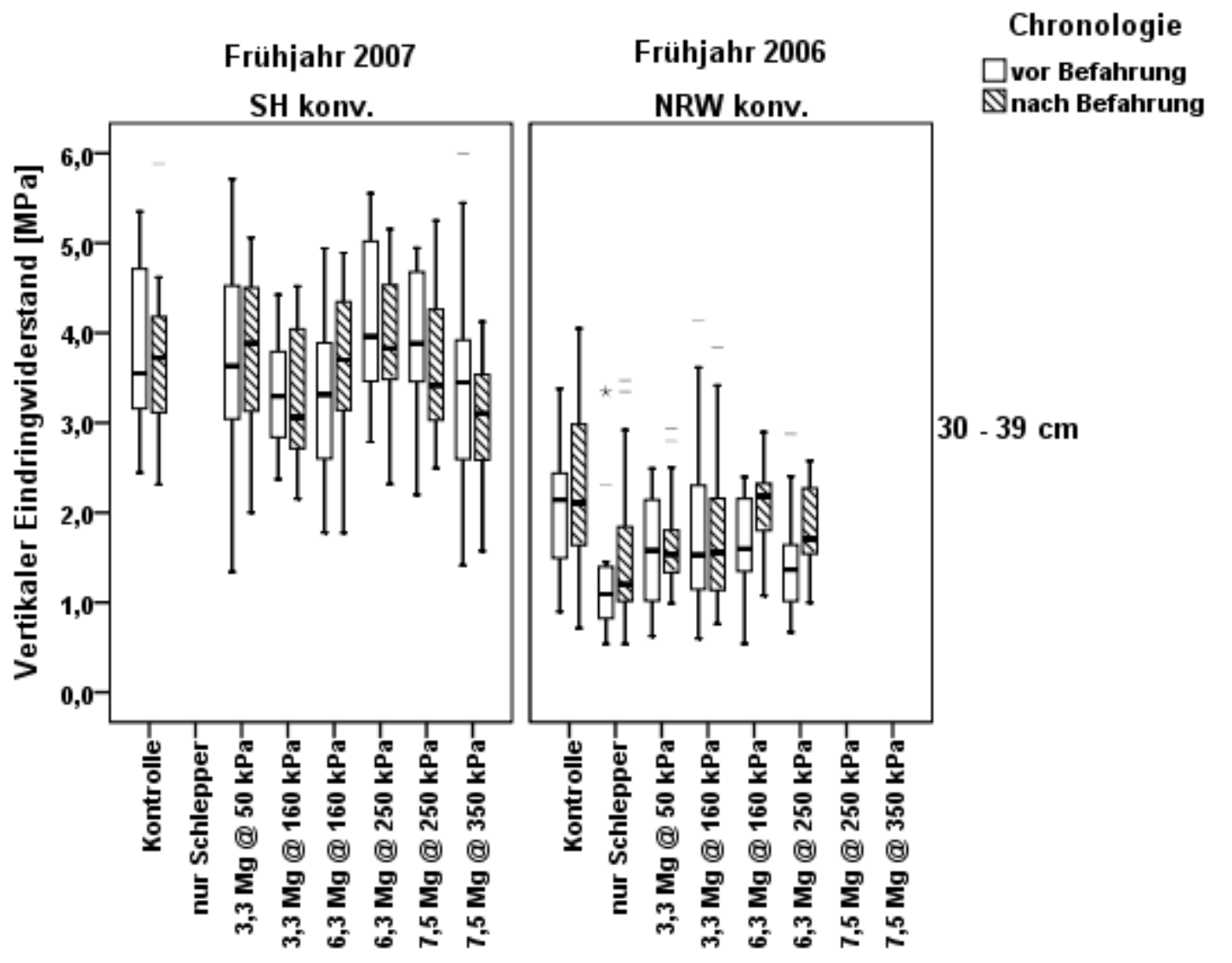

Abbildung 17: Effekte einer Befahrung auf den vertikalen Eindringwiderstand nach zuvor erfolgter wendender Bodenbearbeitung im Messtiefenbereich $30-39 \mathrm{~cm} \quad(n=390$, $\mathrm{GD}_{5 \%} \approx 0,60 \mathrm{MPa}, \overline{\mathrm{x}}$ über 2 Blöcke, $6 \mathrm{Wdh}$.).

Insgesamt lag der vEW am Standort SH im Herbst 2006 zum Zeitpunkt der zweiten VP-Messung um durchschnittlich 19,5\% niedriger als vor der Befahrung, wobei die Abnahme auf $\mathrm{SH}_{\text {kons }}$ mit $-21,0 \%$ deutlicher ausfiel als auf $\mathrm{SH}_{\text {konv }}(-11,5 \%)$. Darüber hinaus waren auf der nicht-wendend bewirtschafteten Fläche die Unterschiede in $5-14 \mathrm{~cm}(-0,48 \mathrm{MPa})$ und $18-27 \mathrm{~cm}(-0,70 \mathrm{MPa})$ deutlicher ausgeprägt als die in den tiefer liegenden Bereichen $30-39 \mathrm{~cm}$ $(-0,24 \mathrm{MPa})$ und $50-59 \mathrm{~cm}(-0,23 \mathrm{MPa})$. Auf $\mathrm{SH}_{\text {konv }}$ hingegen kehrte sich dieses Verhältnis um, und die Abnahme des vEW war umso stärker ausgeprägt, je tiefer die Messungen vorgenommen wurden: während in 5 $14 \mathrm{~cm}$ nahezu unveränderte $\mathrm{vEW}$ gemessen wurden $(-0,01 \mathrm{MPa})$, betrug die Differenz in $50-59 \mathrm{~cm}$ bereits $0,75 \mathrm{MPa}$. 


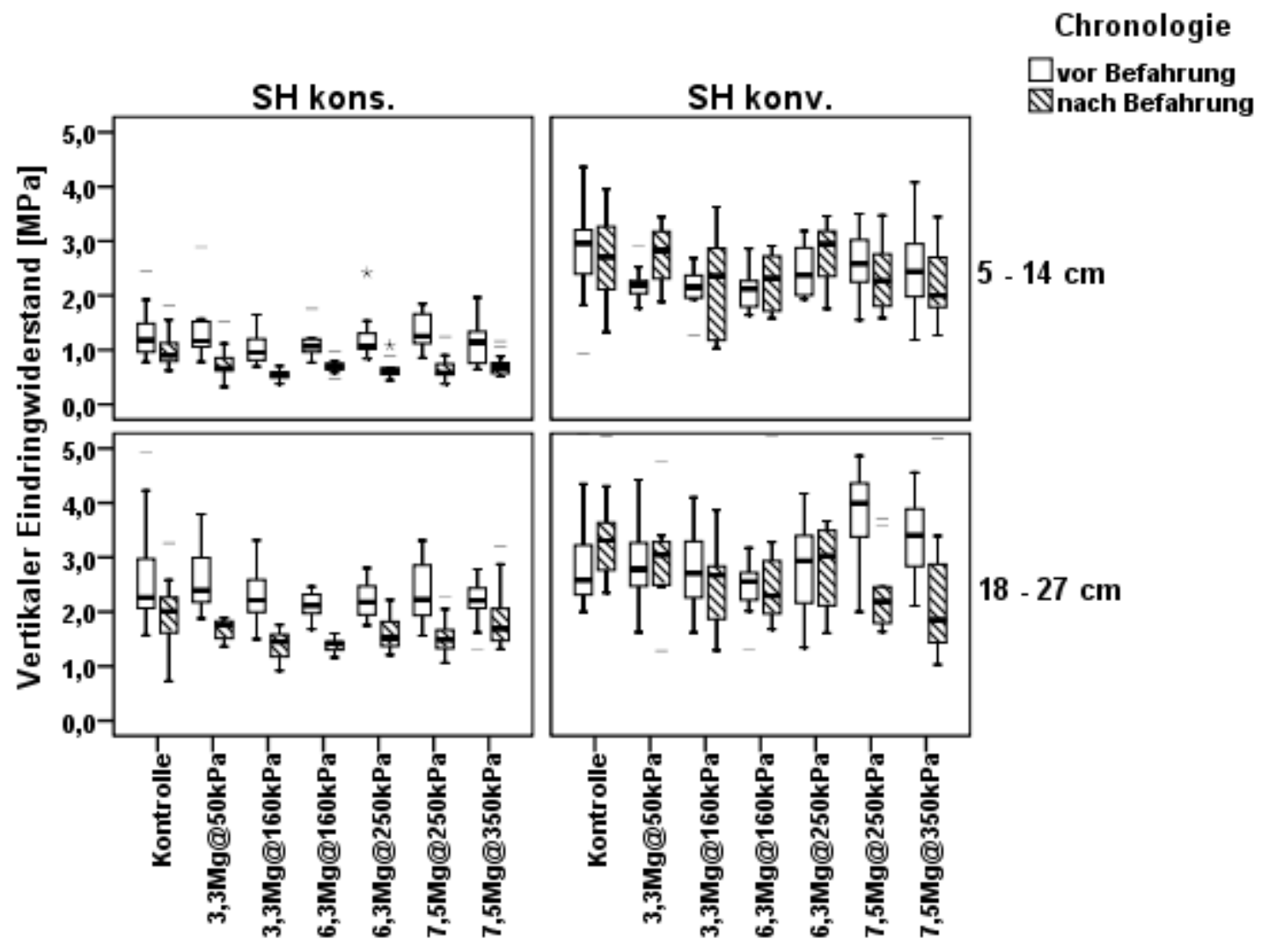

Abbildung 18: Effekte eines Niederschlagsereignisses auf die vertikalen Eindringwiderstände vor und nach einer Befahrung am Standort Schleswig-Holstein im Herbst 2006 in den Messtiefenbereichen $5-14 \mathrm{~cm}$ und $18-27 \mathrm{~cm} \quad(n=704$, $\mathrm{GD}_{5 \%} \approx 0,42 \mathrm{MPa}, \overline{\mathrm{x}}$ über 2 Blöcke, $6 \mathrm{Wdh}$.).

Bei Betrachtung des Bodenwassergehaltes zeigt sich, dass dieser auf $\mathrm{SH}_{\mathrm{kons}}$ an der Bodenoberfläche und in $20 \mathrm{~cm}$ Tiefe zum Zeitpunkt der zweiten Messung um 1,6 \%-Punkte bzw. 1,9\%-Punkte höher lag als bei der Messung zum Zeitpunkt vor der Befahrung. In $40 \mathrm{~cm}$ war hingegen nur ein geringfügiger Unterschied von $0,1 \%$-Punkten festzustellen. Auf $\mathrm{SH}_{\text {konv }}$ lagen die Werte bei der zweiten Beprobung der Parzellen in allen Messtiefen niedriger als bei der ersten Messung. Der deutlichste Unterschied war hier im Bereich der Bodenoberfläche mit einer Abnahme der Bodenfeuchte um 3,4 \%-Punkte zu beobachten (vgl. Tabelle A 18). 


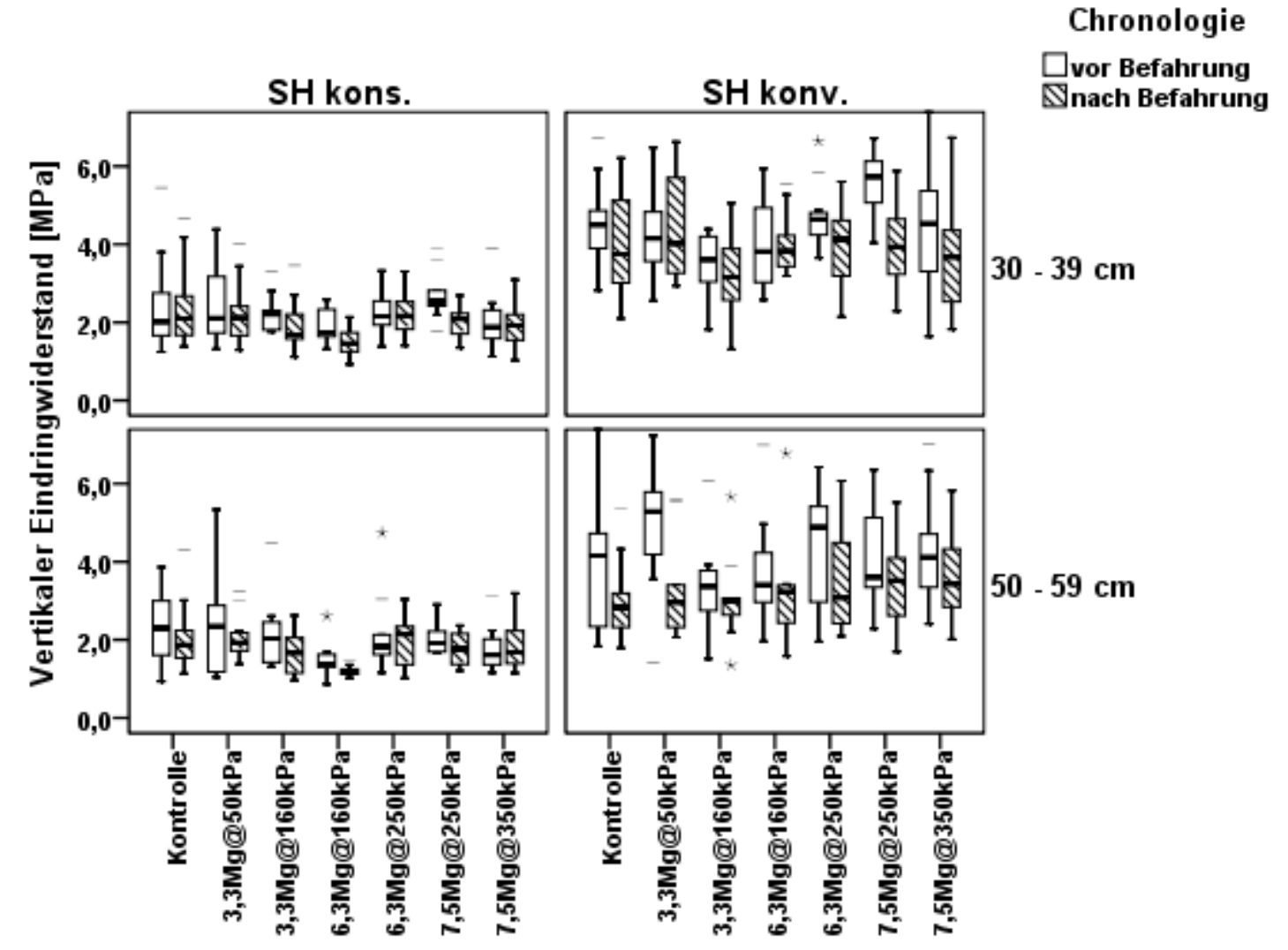

Abbildung 19: Effekte eines Niederschlagsereignisses auf die vertikalen Eindringwiderstände vor und nach einer Befahrung am Standort Schleswig-Holstein im Herbst 2006 in den Messtiefenbereichen $30-39 \mathrm{~cm}$ und 50-59cm $\left(n=703, \mathrm{GD}_{5 \%} \approx 0,73 \mathrm{MPa}\right.$, $\bar{x}$ über 2 Blöcke, 6 Wdh.).

Erkennbar ist, dass die um durchschnittlich -33\% niedrigeren vEW auf $\mathrm{SH}_{\text {kons }}$ in den Messtiefen bis $27 \mathrm{~cm}$ mit steigenden Bodenwassergehalten (+9\%) einhergehen. In Bodenschichten ab $40 \mathrm{~cm}$ sind die Wassergehalte am zweiten Messtermin nur geringfügig erhöht, und die hEW liegen zu diesem Termin geringfügig niedriger.

Auf der konventionell bewirtschaften Fläche lässt sich dieser Zusammenhang nicht herstellen: hier sinken die hEW leicht trotz erfolgter Belastung und niedrigerer Bodenwassergehalte zum zweiten Messzeitpunkt. Angesichts der gemessenen Bodenwassergehalte auf $\mathrm{SH}_{\text {konv }}$ wären höhere $\mathrm{hEW}$ zu erwarten gewesen. Die Schwankungen innerhalb der gemessenen Werte spiegeln aber möglicherweise nur bedingt die hydrologische Dynamik wider, die während der 
Messkampagne im Herbst 2006 vorherrschte, da am Standort SH am 24.10.2006 größere Niederschlagsmengen gemessen wurden. Diese beliefen sich auf $\mathrm{SH}_{\text {konv }}$ auf $40,3 \mathrm{~mm}$ und auf $\mathrm{SH}_{\text {kons }}$ auf $32,6 \mathrm{~mm}$ innerhalb von 24 Stunden. Zum Zeitpunkt der zweiten Messung waren solche Niederschlagsmengen nicht zu verzeichnen.

\subsubsection{Entwicklung des vertikalen Eindringwiderstandes über den Versuchszeitraum}

In Abbildung 20 werden die vEW über den Untersuchungszeitraum dargestellt. Es ist zu erkennen, dass die Werte über die Zeit nicht konstant bleiben, sondern in Abhängigkeit von Fläche, Termin und Messtiefenbereich variieren. Die Flächen $\mathrm{SH}_{\text {kons }}, \mathrm{NRW}_{\text {konv }}$ und $\mathrm{NRW}_{\text {kons }}$ weisen - mit Ausnahmen der untersten Messtiefenbereiche auf den nordrhein-westfälischen Versuchsflächen - die höchsten vEW im Frühjahr 2007 aus. Zu diesem Termin hin steigen sie i. A. an und fallen nachfolgend bis zum Versuchsende wieder ab.

Besonders ausgeprägt ist der Peak des Frühjahres 2007 auf $\mathrm{SH}_{\text {kons }}$ und hier insbesondere in den oberen Messtiefen, während er auf den nordrheinwestfälischen Flächen moderater ausfällt und auf $\mathrm{SH}_{\text {konv }}$ gar nicht zu beobachten ist. Diese Messwerte bewegen sich in enger Übereinstimmung mit den gemessenen Bodenwassergehalten (vgl. Abbildung 11), die im Frühjahr 2007 auf allen Flächen im Oberboden ihre Minima aufweisen, sowie mit den durchgeführten Bodenbearbeitungsmaßnahmen, die diese teilweise überlagern (vgl. Tabelle A 6). 


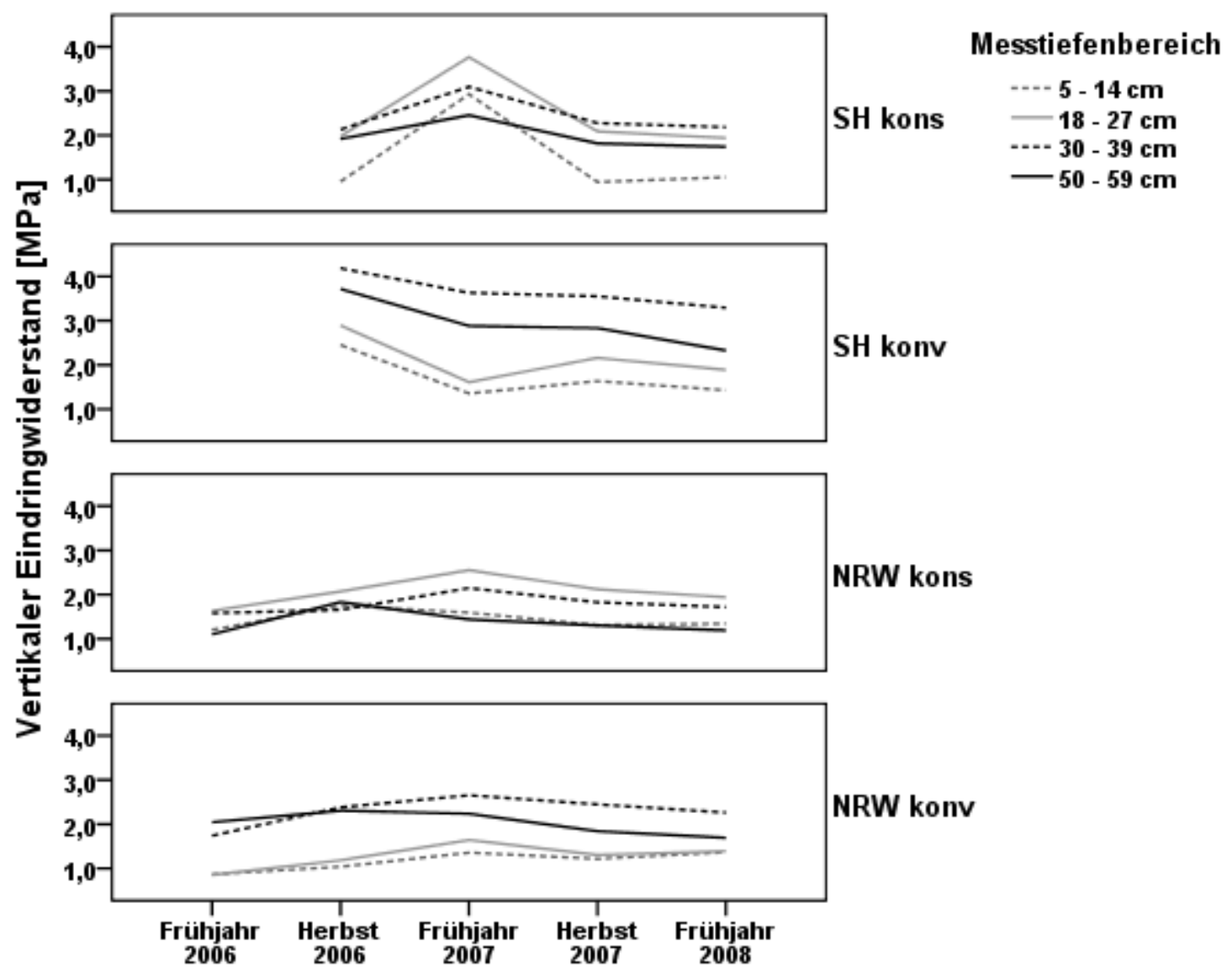

Abbildung 20: Vertikale Eindringwiderstände nach Fläche, Termin und Messtiefenbereich ( $n=15.362, \mathrm{GD}_{5 \%} \approx 0,12 \mathrm{MPa}, \overline{\mathrm{x}}$ über 2 Zeitpunkte, 6 Varianten, 2 Blöcke, $6 \mathrm{Wdh}$.).

Auf der Fläche $\mathrm{SH}_{\text {kons }}$ lag der mittlere vEW des trockenen Frühjahres 2007 mit $3,06 \pm 1,05 \mathrm{MPa}$ signifikant über jenen der drei übrigen kalendarischen Messtermine, die sich mit Werten zwischen 1,73 $\pm 0,67 \mathrm{MPa}$ (Frühjahr 2008) und 1,78 $\pm 0,77 \mathrm{MPa}$ (Herbst 2007) untereinander nicht statistisch signifikant unterschieden. Der Gesamtmittelwert von $\mathrm{SH}_{\text {kons }}$ über den Versuchzeitraum lag bei 2,08 $\pm 1,01 \mathrm{MPa}$. Höhere Messwerte zum Frühjahrstermin 2007 sind in allen Messtiefenbereichen zu beobachten, wobei die Ausprägung mit zunehmender Bodentiefe abnimmt: der Oberboden $(5-14 \mathrm{~cm})$ weist einen um $+99,1 \%$ erhöhten vEW gegenüber dem Gesamtmittel der Fläche im entsprechenden Messtiefenbereich auf; im nahen Oberboden (18 - $27 \mathrm{~cm}$ ) beträgt der Anstieg $+54,5 \%$; im nahen Unterboden $(30-39 \mathrm{~cm})$ sind es noch $+27,6 \%$ und im Unterboden $(50-59 \mathrm{~cm})+23,7 \%$. Am Ende des Untersuchungszeitraumes 
weisen die Messwerte in keinem Messtiefenbereich einen signifikanten Unterschied gegenüber dem ersten Messtermin auf.

$\mathrm{SH}_{\text {konv }}$ lässt im Herbst 2006 ein hohes Ausgangsniveau der Messwerte erkennen, das über den Untersuchungszeitraum in den beiden unteren Messtiefenbereichen kontinuierlich abnimmt. Die niedrigsten Werte in den oberen beiden Bodenschichten weist das Frühjahr 2007 aus, was auf die zuvor besprochene wendende Bodenbearbeitung zurückzuführen ist, deren Messwerte vor der Befahrung mit berücksichtigt sind.

Wie auf $\mathrm{SH}_{\text {konv }}$ so unterscheiden sich auch auf $\mathrm{NRW}_{\text {kons }}$ die Daten aller einzelnen kalendarischen Messtermine signifikant untereinander. Nach niedrigen Ausgangswerten zu Versuchsbeginn erreichen die vEW im Frühjahr 2007 in allen Messtiefenbereichen mit durchschnittlich 1,93 \pm 0,75 MPa ihr Maximum, um danach wieder leicht abzufallen. Mit 1,55 $\pm 0,47 \mathrm{MPa}$ bewegen sie sich auf einem Niveau, das $+12,1 \%$ oberhalb des Ausgangsniveaus liegt.

Die Messwerte von $\mathrm{NRW}_{\text {konv }}$ lagen bei durchschnittlich 1,71 \pm 0,78 MPa und damit höher als die Werte der konservierend bewirtschafteten Fläche in NRW. Die Messungen Herbst 2006 (1,73 \pm 0,84 MPa), Herbst $2007(1,70 \pm$ 0,69 MPa) und Frühjahr 2008 (1,68 \pm 0,63 MPa) unterschieden sich in ihren Mittelwerten nicht signifikant voneinander, wohl aber von jenen der Frühjahre 2006 $(1,38 \pm 0,79 \mathrm{MPa})$ und $2007(1,97 \pm 0,84 \mathrm{MPa})$. Auch auf $\mathrm{NRW}_{\mathrm{konv}}$ werden die höchsten vEW im Frühjahr 2007 verzeichnet: sie liegen etwa +0,2 MPa über jenen des nachfolgenden Termins und dem Herbst 2006, die Differenz zum Frühjahrs 2006 beträgt +0,59 MPa. Das Ausgangsniveau in den beiden oberen Messtiefen ist mit < $1 \mathrm{MPa}$ das niedrigste auf allen Versuchsflächen. Auch zu einem späteren Zeitpunkt wird ein Wert von 1,7 MPa nicht überschritten. Der Messtiefenbereich $30-39 \mathrm{~cm}$ weist mit durchschnittlich 2,30 \pm 0,50 MPa den höchsten Wert aus. Während die Werte für die drei oberen Messtiefenbereiche bis zum Frühjahr 2007 ansteigen und danach stetig abfallen, ist dieses im Bereich $50-59 \mathrm{~cm}$ bereits nach dem Maximum im Herbst 2006 $(2,31 \pm 0,37 \mathrm{MPa})$ zu beobachten. 


\subsubsection{Effekte einer mechanischen Belastung}

Wie in Kapitel 4.4.2 dargelegt, resultieren Befahrungen nicht zwangsläufig in einer Erhöhung des vEW. Gemittelt über die Varianten konnte in 6 von 18 Fällen (Fläche * Termin) nach den Befahrungen keine signifikante Änderung des EW gegenüber der Messung vor der mechanischen Belastung festgestellt werden. In sieben der untersuchten Fälle wurden signifikant höhere vEW nachgewiesen, und bei vier Messereignissen - zwei von denen bereits oben behandelt wurden - ergab sich nach der Befahrung sogar ein signifikant niedrigerer vEW. Die Fälle erniedrigter vEW nach einer Belastung sind auf die schleswig-holsteinischen Versuchsflächen beschränkt. Neben den bereits beschriebenen Ergebnissen des Herbstes 2006 konnte auch im Frühjahr 2008 diese Beobachtung gemacht werden, wenn auch in weniger deutlicher Ausprägung. Umgekehrt waren signifikant erhöhte vEW bis auf zwei Messreihen $\left(\mathrm{SH}_{\text {konv }}\right.$ im Frühjahr und Herbst 2007) auf die nordrhein-westfälischen Versuchsflächen beschränkt. 
Tabelle 5: Vertikale Eindringwiderstände in Abhängigkeit des Zeitpunktes der Beprobung ( $n=15.362, \bar{x}$ über 4 Messtiefenbereiche (MTB), 6 Varianten, 2 Blöcke, 6 Wdh.)

\begin{tabular}{|c|c|c|c|c|c|c|}
\hline Fläche/ Termin & $\begin{array}{l}\text { vEW } \\
\text { vor } \\
\text { Bef. } \\
\text { [MPa] }\end{array}$ & $\begin{array}{l}\text { vEW } \\
\text { nach } \\
\text { Bef. } \\
\text { [MPa] }\end{array}$ & $\begin{array}{l}\Delta \mathrm{vEW} \\
(\triangleq \text { Trend })\end{array}$ & $\begin{array}{l}\text { Maßgebliche }{ }^{1} \\
\text { Varianten, für die } \\
\text { Trend gültig }\end{array}$ & $\begin{array}{l}\text { Maßgebliche }{ }^{1} \\
\text { MTB, für die } \\
\text { Trend gültig }\end{array}$ & $\begin{array}{l}\Delta \mathrm{W}_{\mathrm{g}} \\
{[\%-} \\
\text { Punkte] }\end{array}$ \\
\hline \multicolumn{7}{|l|}{$\mathrm{SH}_{\text {kons }}$} \\
\hline Herbst $2006^{a}$ & 1,97 & 1,56 & $-0,41^{*}$ & alle & alle & $+1,1$ \\
\hline Frühjahr $2007^{b}$ & 3,08 & 3,04 & $-0,04$ & $--^{2}$ & $5-14 \mathrm{~cm}$ & $+0,7$ \\
\hline Herbst $2007^{a}$ & 1,82 & 1,74 & $-0,08$ & $\begin{array}{l}\text { 3,3Mg@50kPa } \\
6,3 \mathrm{Mg} @ 160 \mathrm{kPa} \\
7,5 \mathrm{Mg} @ 350 \mathrm{kPa}\end{array}$ & $\begin{array}{l}5-14 \mathrm{~cm} \\
30-39 \mathrm{~cm}\end{array}$ & $-0,5$ \\
\hline Frühjahr $2008^{a}$ & 1,79 & 1,68 & $-0,11^{*}$ & $\begin{array}{l}\text { 6,3Mg@160kPa } \\
6,3 \mathrm{Mg} @ 250 \mathrm{kPa} \\
7,5 \mathrm{Mg} @ 250 \mathrm{kPa} \\
7,5 \mathrm{Mg} @ 350 \mathrm{kPa}\end{array}$ & $5-14 \mathrm{~cm}$ & $-1,2$ \\
\hline $\begin{array}{l}\text { gesamt } \\
\text { SH }_{\text {konv }}\end{array}$ & $\underline{2,17}$ & $\underline{1,99}$ & $\underline{-0,18^{*}}$ & & & \\
\hline Herbst $2006^{a}$ & 3,49 & 3,09 & $-0,40^{*}$ & $\begin{array}{l}\text { nicht für: } \\
\text { 6,3Mg@160kPa }\end{array}$ & $\begin{array}{l}\text { nicht für: } \\
5-14 \mathrm{~cm}\end{array}$ & $-2,0$ \\
\hline Frühjahr $2007^{b}$ & 2,21 & 2,52 & $+0,31^{*}$ & $\begin{array}{l}\text { nicht für: } \\
\text { Kontrolle }\end{array}$ & $\begin{array}{l}\text { nicht für: } \\
30-39 \mathrm{~cm}\end{array}$ & $+0,4$ \\
\hline Herbst $2007^{b}$ & 2,42 & 2,63 & $+0,21^{*}$ & $\begin{array}{l}\text { 3,3Mg@50kPa } \\
6,3 \mathrm{Mg} @ 250 \mathrm{kPa} \\
7,5 \mathrm{Mg} @ 350 \mathrm{kPa}\end{array}$ & alle & $\pm 0,0$ \\
\hline Frühjahr $2008^{b}$ & 2,33 & 2,14 & $-0,20^{*}$ & $\begin{array}{l}\text { Kontrolle } \\
6,3 \mathrm{Mg} @ 250 \mathrm{kPa} \\
7,5 \mathrm{Mg} @ 250 \mathrm{kPa} \\
7,5 \mathrm{Mg} @ 350 \mathrm{kPa}\end{array}$ & alle & $-1,1$ \\
\hline gesamt & $\underline{2,62}$ & $\underline{2,58}$ & $-0,04$ & & & \\
\hline
\end{tabular}




\begin{tabular}{|c|c|c|c|c|c|c|}
\hline Fläche/ Termin & $\begin{array}{l}\text { vEW } \\
\text { vor } \\
\text { Bef. } \\
\text { [MPa] }\end{array}$ & $\begin{array}{l}\text { vEW } \\
\text { nach } \\
\text { Bef. } \\
\text { [MPa] }\end{array}$ & $\begin{array}{l}\Delta \mathrm{vEW} \\
(\stackrel{\wedge}{=} \text { Trend })\end{array}$ & $\begin{array}{l}\text { Maßgebliche }^{1} \\
\text { Varianten, für die } \\
\text { Trend gültig }\end{array}$ & $\begin{array}{l}\text { Maßgebliche }{ }^{1} \\
\text { MTB, für die } \\
\text { Trend gültig }\end{array}$ & $\begin{array}{l}\Delta \mathrm{W}_{\mathrm{g}} \\
{[\%-} \\
\text { Punkte] }\end{array}$ \\
\hline \multicolumn{7}{|l|}{ NRW $_{\text {kons }}$} \\
\hline Frühjahr $2006^{a}$ & 1,42 & 1,33 & $-0,08$ & 6,3Mg@250kPa & $\begin{array}{l}5-14 \mathrm{~cm} \\
30-39 \mathrm{~cm}\end{array}$ & $+0,1$ \\
\hline Herbst $2006^{b}$ & 1,70 & 1,97 & $+0,26^{*}$ & $\begin{array}{l}\text { nicht für: } \\
\text { 6,3Mg@250kPa }\end{array}$ & $\begin{array}{l}\text { nicht für: } \\
50-59 \mathrm{~cm}\end{array}$ & $-1,2$ \\
\hline Frühjahr $2007^{c}$ & 1,81 & 2,05 & $+0,24^{*}$ & $\begin{array}{l}\text { nicht für: } \\
\text { Kontrolle } \\
\text { 6,3Mg@160kPa }\end{array}$ & $\begin{array}{l}\text { nicht für: } \\
50-59 \mathrm{~cm}\end{array}$ & $-1,0$ \\
\hline Herbst $2007^{d}$ & 1,64 & 1,64 & $+0,01$ & $--^{2}$ & $--^{2}$ & $+1,0$ \\
\hline Frühjahr $2008^{\mathrm{e}}$ & 1,57 & 1,53 & $-0,04$ & $--^{2}$ & $5-14 \mathrm{~cm}$ & $-0,7$ \\
\hline gesamt & $\underline{1,63}$ & $\underline{1,72}$ & $+0,09$ & & & \\
\hline NRW $_{\text {konv }}$ & & & & & & \\
\hline Frühjahr $2006^{a}$ & 1,17 & 1,61 & $+0,44^{*}$ & alle & alle & $+1,0$ \\
\hline Herbst $2006^{b}$ & 1,63 & 1,83 & $+0,20^{*}$ & $\begin{array}{l}\text { nicht für: } \\
\text { 3,3Mg@50kPa }\end{array}$ & $\begin{array}{l}\text { nicht für: } \\
50-59 \mathrm{~cm}\end{array}$ & $-1,5$ \\
\hline Frühjahr $2007^{c}$ & 1,79 & 2,16 & $+0,38^{*}$ & alle & alle & $-0,9$ \\
\hline Herbst $2007^{b}$ & 1,68 & 1,73 & $+0,05$ & 3,3Mg@160kPa & $\begin{array}{l}5-14 \mathrm{~cm} \\
18-27 \mathrm{~cm}\end{array}$ & $-0,2$ \\
\hline Frühjahr $2008^{b}$ & 1,69 & 1,66 & $-0,03$ & $--^{2}$ & $--^{2}$ & $\pm 0,0$ \\
\hline gesamt & $\underline{1,61}$ & $\underline{1,81}$ & $+0,20^{*}$ & & & \\
\hline
\end{tabular}

Unterschiedliche Buchstaben kennzeichnen signifikante Unterschiede mit $p \leq 0,05$. Sie gelten für die jeweilige Spalte und sind im Falle der kalendarischen Termine flächenbezogen.

* Die mittlere Differenz (,vor der Befahrung“vs. ,nach der Befahrung“) ist signifikant mit $\mathrm{p} \leq 0,05$.

${ }^{1}$ Bedingung: Betrag der Differenz (,nach der Befahrung“ - ,,vor der Befahrung“) $>0,1 \mathrm{MPa}$.

${ }^{2}$ Keine Angabe bei: $\Delta \mathrm{vEW}<0,1 \mathrm{MPa}$ und nicht signifikanter Ausprägung oder stark uneinheitlichem Trend

Die deutlichsten Abnahmen der vEW wurden im Herbst 2006 auf SH $_{\text {kons }}$ (-0,41 MPa) und $\mathrm{SH}_{\text {konv }}(-0,40 \mathrm{MPa})$ festzustellen, die deutlichsten Erhöhungen 
auf $\mathrm{NRW}_{\text {konv }}$ im Frühjahr $2006(+0,44 \mathrm{MPa})$ und Frühjahr 2007 (+0,38 MPa) sowie auf $\mathrm{SH}_{\text {konv }}$ ebenfalls im Frühjahr 2007 (+0,31 MPa).

Bei denjenigen Ereignissen, bei denen witterungsbedingt eine signifikante Abnahme des vEW zu beobachten war $\left(\mathrm{SH}_{\text {kons }}\right.$ und $\mathrm{SH}_{\text {konv }}$ im Herbst 2006), konnte dieser Trend für nahezu alle Varianten einschließlich der Kontrolle und in allen Messtiefenbereichen bestätigt werden (vgl. Tabelle 5). Bei den Messungen auf $\mathrm{SH}_{\text {konv }}$ im Frühjahr 2008, blieben diese Beobachtungen auf die schweren Varianten ab 6,3Mg@250kPa beschränkt. Aber auch hier war der

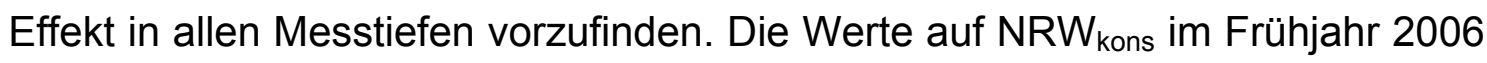
bewegten sich an der statistischen Signifikanzgrenze und waren auf die Variante 6,3Mg@250kPa und die Messtiefenbereiche 5 - $14 \mathrm{~cm}$ und 30 - $39 \mathrm{~cm}$ beschränkt.

Neben den bereits behandelten Messreihen nach unmittelbar zuvor erfolgter Bodenbearbeitung konnten auch auf $\mathrm{SH}_{\text {konv }}$ im Herbst 2007 signifikant höhere vEW nach den durchgeführten Befahrungen gemessen werden. Obwohl für mehrere Varianten und Messtiefen ein höherer vEW nach der Befahrung festgestellt wurde, konnten die Ergebnisse nur für die Variante 7,5Mg@350kPa bzw. den Messtiefenbereich 5-14 cm als statistisch signifikant abgesichert werden. Anders verhielt es sich bei den Messungen auf $\mathrm{NRW}_{\text {kons }}$ im Herbst 2006, bei denen lediglich die Variante 6,3Mg@250kPa und die Messtiefe 50 $59 \mathrm{~cm}$ nicht als statistisch signifikant abgesichert wurden. Auch bei dieser Messreihe war aber der deutlichste Anstieg der VEW (jeweils +0,50 MPa) in den oberen Messtiefen bis $27 \mathrm{~cm}$ zu beobachten. In $30-39 \mathrm{~cm}$ fiel der vEW mit $+0,20 \mathrm{MPa}$ bereits geringer aus, und in $50-59 \mathrm{~cm}$ war keine Zunahme zu verzeichnen. Auch im Frühjahr 2007 konnte eine signifikante Erhöhung der vEW auf dieser Fläche nur bis zu einer Tiefe von $39 \mathrm{~cm}$ festgestellt werden, nicht jedoch für die Kontrolle, deren Wert unverändert blieb ebenso wie der Wert der Variante 6,3Mg@160kPa.

Auf NRW konv konnten in den Frühjahren 2006 und 2007 sowie im Herbst 2006 an drei aufeinander folgenden Terminen nach den durchgeführten Befahrungen signifikant höhere VEW für fast alle Varianten und Messtiefen gemessen werden. Mit zunehmender Bodentiefe sank jedoch die relative Zunahme des 
vEW. So lag der Wert in der Schicht $5-14 \mathrm{~cm}$ noch um durchschnittlich $+65,5 \%$ über dem Ausgangswert, während die Differenz in der Messtiefe 18 - $27 \mathrm{~cm}$ nur noch $+40,3 \%$ und im Bereich der Pflugsohle nur noch $+10,9 \%$ betrug. Im Bereich des Unterbodens konnte nur noch eine Zunahme des vEW von $+7,3 \%$ gegenüber dem gemittelten Ausgangswert vor den Befahrungen ermittelt werden.

Im nachfolgenden Herbst 2007 nahm der vEW nur noch in den obersten beiden Messbereichen und für die Variante 3,3@160kPa signifikant zu, in tieferen Schichten und zum späteren Messtermin 2008 erhöhte er sich hingegen nur noch geringfügig. Die Messwerte des Frühjahres 2008 zeigen - auch auf anderen Flächen - insgesamt eine geringe Dynamik.

Die Entwicklung der vEW geht nur unvollständig mit höheren bzw. niedrigeren Bodenwassergehalten einher. Auf den schleswig-holsteinischen Versuchsflächen konnte ein Zusammenhang mehrmals beobachtet werden: bei niedrigeren Bodenwassergehalten wurden auch niedrigere hEW gemessen und umgekehrt. Für die nordrhein-westfälischen Flächen schien dieser Zusammenhang mit einer noch höheren Übereinstimmung hergestellt werden zu können. Einzig auf NRW konv im Frühjahr 2006 war eine deutlich konträre Entwicklung der beiden Messwerte zu erkennen. Hierbei sollten jedoch versuchstechnische und methodische Bedingungen mit berücksichtigt werden.

\subsubsection{Einflüsse der Fahrwerksparameter Radlast und Reifeninnendruck}

Nachdem in den vorherigen Kapiteln dargestellt wurde, dass mit dem VP Einflüsse langjähriger Bewirtschaftungspraktiken, besonderer Niederschlagsereignisse sowie mechanischer Belastungen nach unmittelbar zuvor erfolgter Bodenbearbeitung festgestellt werden können, soll im nächsten Schritt untersucht werden, ob die Methode auch geeignet ist, differenzierte Aussagen über dichter lagernde Böden nach unterschiedlich starken mechanischen Belastungen, wie sie im Versuchsdesign berücksichtigt sind, zu erfassen. Das Versuchsdesign erlaubt es, den Datensatz dahingehend $z u$ analysieren, wie sich die vEW zwischen Varianten unterschiedlicher Radlasten, aber identischer 
Reifeninnendrücke (und vice versa) unterscheiden. Betrachtet werden nur VPDaten nach erfolgter Befahrung.

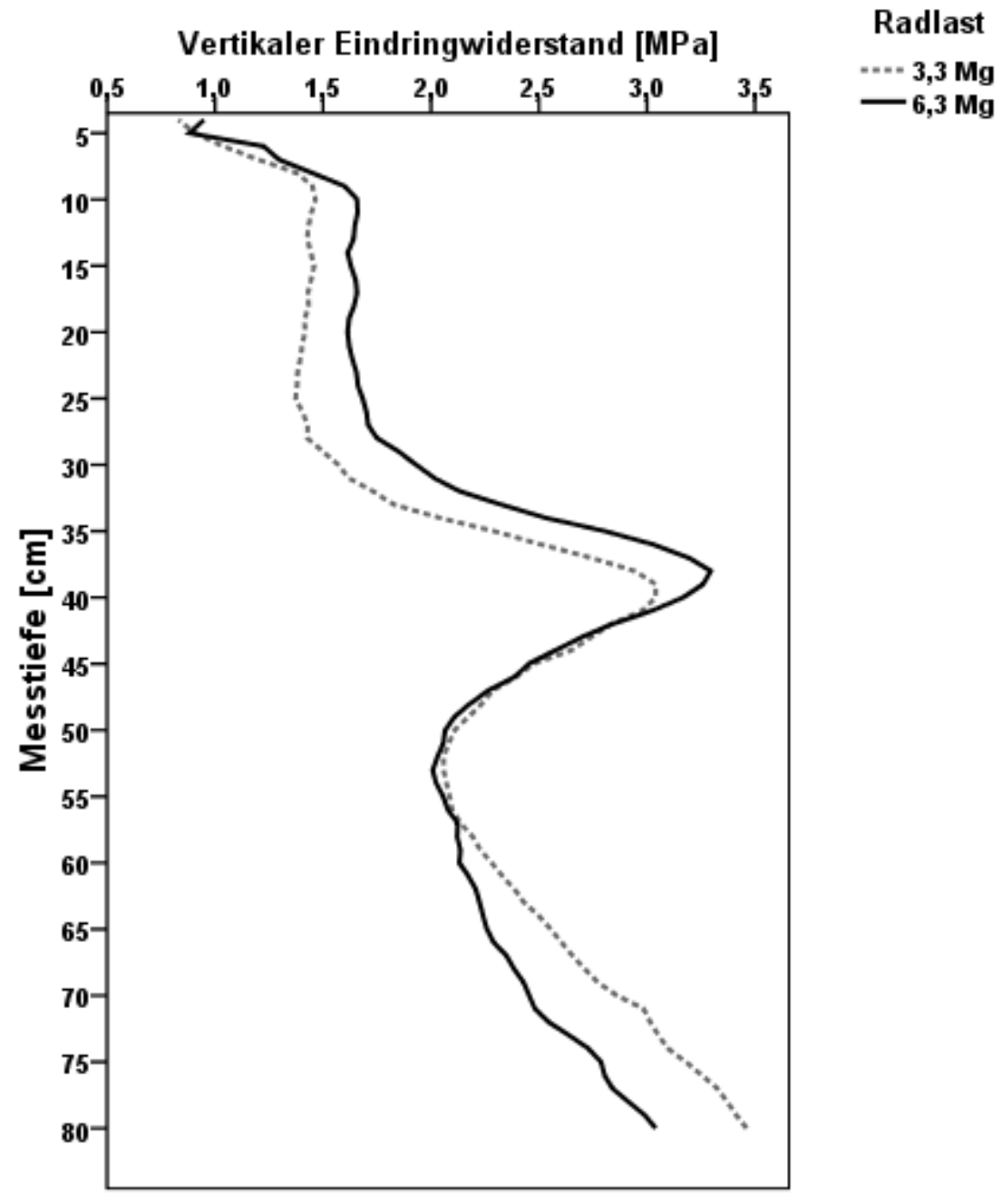

Abbildung 21: Vertikale Eindringwiderstände [MPa] nach erfolgter Befahrung mit unterschiedlichen Radlasten bei konstantem Reifeninnendruck (160 kPa) auf $\mathrm{NRW}_{\mathrm{konv}}$ ( $n=180, \bar{x}$ über 5 Termine, 3 Blöcke, 6 Wdh.).

Beim Vergleich der Varianten 3,3Mg@160kPa (1,90 \pm 0,84 MPa) und 6,3Mg@160kPa (1,97 \pm 0,88 MPa) im Gesamtmittel zeigte sich zunächst, dass zwischen ihnen kein statistisch signifikanter Unterschied bestand. Erst unter Berücksichtigung der unterschiedlichen Flächen und Messtiefenbereiche stellte sich heraus, dass 5 unter 16 untersuchten Ereignissen statistisch unterscheidbar waren. Drei von ihnen wurden auf $\mathrm{NRW}_{\text {konv }}$ in den Messtiefen zwischen $5 \mathrm{~cm}$ und $39 \mathrm{~cm}$ beobachtet. In einem Bereich von $40 \mathrm{~cm}$ bis $65 \mathrm{~cm}$ bestanden nahezu keine Unterschiede zwischen den beiden Radlastvarianten (vgl. 
Abbildung 21). Unterhalb $65 \mathrm{~cm}$ Bodentiefe kehrte sich das Verhältnis der hEW um, allerdings nicht in einem statistisch signifikanten Maße. Mit einem um durchschnittlich 0,20 MPa höherem vEW bei der höherer Radlast zeigte sich dieser Faktor auf $\mathrm{NRW}_{\text {konv }}$ auch insgesamt als statistisch signifikant (vgl. Tabelle A 20).

Anders verhielt es sich bei der untersuchten Radlasterhöhung von 6,3 Mg auf 7,5 Mg. Hier konnte auf keiner Fläche und in keiner Messtiefe ein signifikanter Unterschied zwischen den Varianten mit $250 \mathrm{kPa}$ Reifeninnendruck nachgewiesen werden. Bezogen auf die Versuchsflächen betrug der maximale

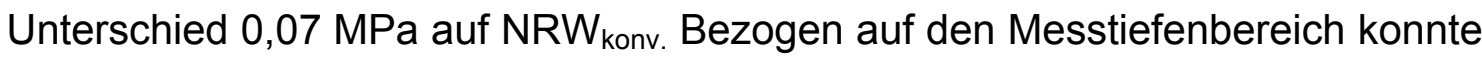
bei 30 - $39 \mathrm{~cm}$ eine maximale Differenz von 0,10 MPa festgestellt werden (vgl. Tabelle A 21).

Die Betrachtung der Effekte einer Erhöhung des Reifeninnendruckes bei den unterschiedlichen Radlastvarianten zeigt, dass diese - über die Flächen und Messtiefenbereiche gemittelt - keinen nennenswerten Einfluss auf den vEW ausüben: der Unterschied zwischen dem höheren und niedrigeren Reifeninnendruck beträgt maximal 0,06 MPa. Eine weitergehende Untersuchung der Daten nach Fläche und Messtiefe ergibt einzelne Flächen-Messtiefen-Konstellationen, die als statistisch signifikant ausgewiesen werden (vgl. Tabelle A 23 und Tabelle A 24). So führt eine Erhöhung des RID von $160 \mathrm{kPa}$ auf $250 \mathrm{kPa}$ bei $6,3 \mathrm{Mg}$ Radlast in Messtiefe $50-59 \mathrm{~cm}$ auf $\mathrm{SH}_{\text {konv }} \mathrm{zu}$ einer signifikanten Zunahme des vEW. Auch in den darüber liegenden Messtiefenbereichen können auf $\mathrm{SH}_{\text {konv }}$ jeweils höhere vEW bei höherem RID festgestellt werden, so dass auf dieser Fläche auch im Gesamtmittel über alle Messtiefen ein signifikanter Unterschied zwischen den RID-Varianten bei einer Radlast von 6,3 Mg besteht. Auch auf $\mathrm{SH}_{\text {kons }}$ steigen die hEW in der Tiefe von $50-59 \mathrm{~cm}$ mit einer Erhöhung des RID signifikant an, was in der Tendenz auch für den Messtiefenbereich 30-39 cm zutrifft. Die oberen Bereiche weisen entweder keine Unterschiede $(18-27 \mathrm{~cm})$ oder eine leichte Abnahme der hEW mit steigendem RID (5 - $14 \mathrm{~cm})$ auf. 
Fläche

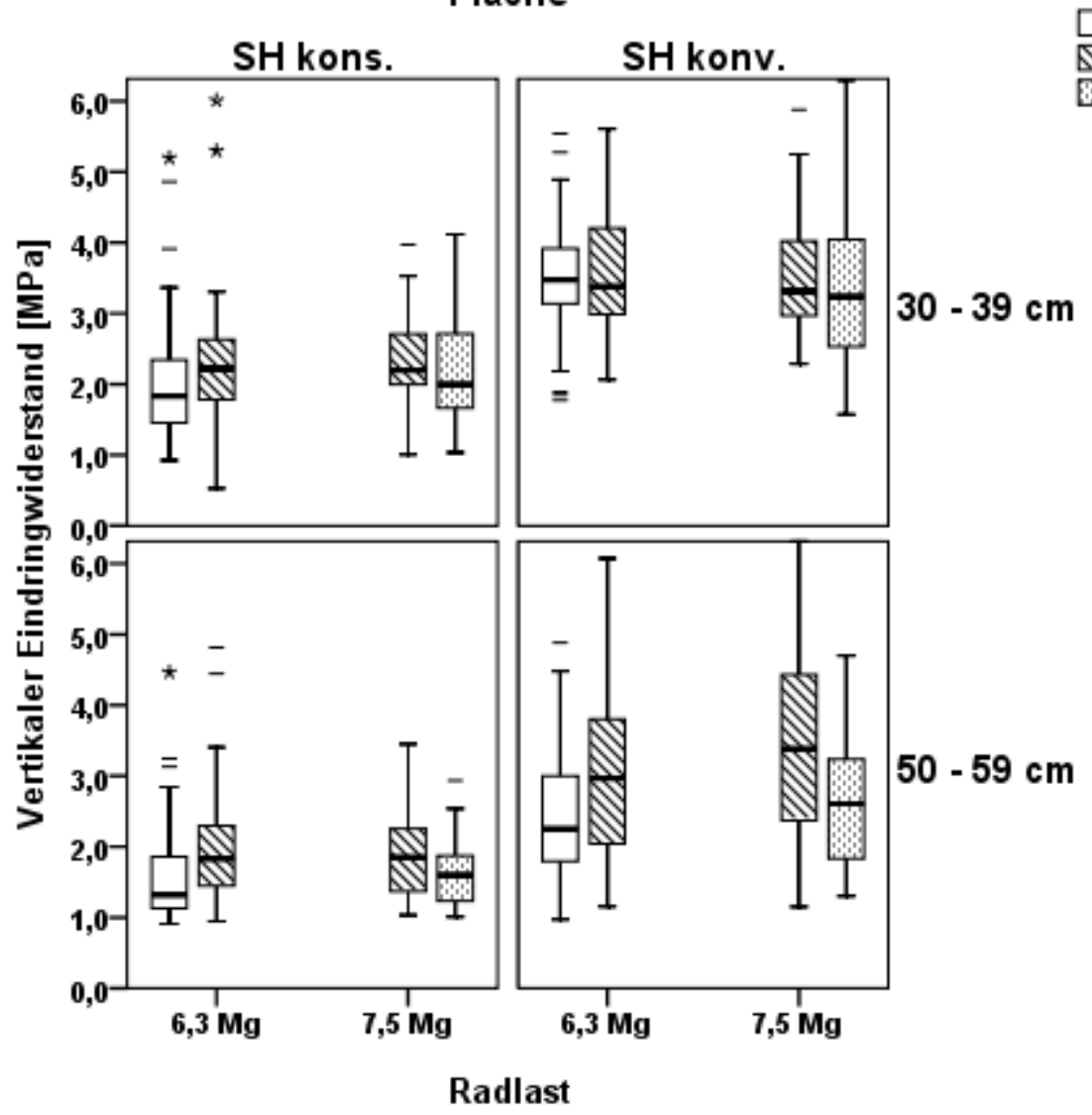

Reifendruck

$\square 160 \mathrm{kPa}$

$\triangle 250 \mathrm{kPa}$

웅 $350 \mathrm{kPa}$

Abbildung 22: Vertikale Eindringwiderstände [MPa] bei konstanten Radlasten und unterschiedlichen Reifeninnendrücken am Standort Schleswig-Holstein in den Messtiefenbereichen $30-39 \mathrm{~cm}$ und $50-59 \mathrm{~cm}\left(n=186, \mathrm{GD}_{5 \%} \approx 0,37 \mathrm{MPa}, \overline{\mathrm{x}}\right.$ über 2 Blöcke, 6 Wdh.).

Die gegenläufige Beobachtung kann auf denselben Flächen bei einer RIDÄnderung von $250 \mathrm{kPa}$ auf $350 \mathrm{kPa}$ und 7,5 Mg Radlast gemacht werden (vgl. Abbildung 22): während auf $\mathrm{SH}_{\text {konv }}$ bei höherem RID tendenziell niedrigere $\mathrm{hEW}$ in allen Messtiefenbereichen festgestellt werden können, ist dieses auf $\mathrm{SH}_{\text {kons }}$ nur in den beiden unteren Messtiefen der Fall. In 50-59 cm können am Standort $\mathrm{SH}$ bei beiden Bodenbewirtschaftungsverfahren signifikant niedrigere hEW bei höherem RID und 7,5 Mg Radlast beobachtet werden, wobei die Unterschiede auf $\mathrm{SH}_{\text {konv }}$ mit $0,69 \mathrm{MPa}$ deutlicher ausfallen als auf $\mathrm{SH}_{\text {kons }}$ $(0,30 \mathrm{MPa})$.

Auf $\mathrm{NRW}_{\text {kons }}$ sind bei einer RID-Erhöhung für $6,3 \mathrm{Mg}$ Radlast tendenziell niedrigere $\mathrm{hEW}$ in jedem der Messtiefenbereiche erkennbar, die im Gesamt- 
mittel für die Fläche auch als statistisch signifikant ausgewiesen werden. Allerdings können vergleichbare Trends weder für die RID-Varianten mit 7,5 Mg Radlast auf $\mathrm{NRW}_{\text {kons }}$ noch für RID-Änderungen bei $6,3 \mathrm{Mg}$ und 7,5 Mg Radlast auf $\mathrm{NRW}_{\text {konv }}$ beobachtet werden.

\subsection{Horizontaler Eindringwiderstand}

Mit dem Messsystem TASIS wurden an drei Terminen Messungen auf den Versuchsflächen jeweils nach den Befahrungen durchgeführt. Nach den ersten Messungen in 2006 wurden auf Basis der ersten Ergebnisse sowohl Hardwareals auch Software-Komponenten des Systems mit dem Ziel optimiert, in den folgenden Kampagnen differenziertere Datensätze gewinnen zu können.

\subsubsection{Datensatzstruktur und Effekte der Hauptfaktoren}

Wie bereits beim Datensatz der VP-Messungen, so zeigte auch die statistische Auswertung der horizontalen Eindringwiderstände (hEW) signifikante Unterschiede zwischen den Standorten und den Bodenbearbeitungsverfahren sowie den kalendarischen Terminen und den Messtiefenbereichen. Für die Faktoren Radlast und Reifeninnendruck konnte in der Gesamtbewertung keine signifikante Bedeutung ermittelt werden. Insgesamt lagen die hEW mit durchschnittlich 0,86 \pm 0,33 MPa erkennbar niedriger als die vEW (1,94 \pm 0,87 MPa). Auch streuten die Daten in erkennbar geringerem Maße. 
Tabelle 6: Horizontale Eindringwiderstände [MPa] nach Fläche und Bodenbearbeitung ( $\bar{x}$ über 3 Termine, 3 Messtiefen, 6 Varianten, 2 Blöcke, 6 Wdh.)

\begin{tabular}{lllllll}
\hline Standort & $\mathrm{BB}$ & $\mathrm{n}$ & $\mathrm{M}$ & $\mathrm{SD}$ & $\mathrm{SED}$ & $\mathrm{GD}_{5 \%}$ \\
\hline NRW & kons. & 972 & 0,87 & 0,20 & 0,01 & 0,02 \\
& konv. & 1.296 & 0,75 & 0,23 & 0,01 & 0,02 \\
& $\underline{\text { Insgesamt }}$ & $\underline{2.268}$ & $\underline{0,80}$ & $\underline{0,22}$ & $\underline{0,00}$ & $\underline{0,01}$ \\
$\mathrm{SH}$ & kons. & 822 & 0,89 & 0,36 & 0,01 & 0,03 \\
& konv. & 765 & 1,00 & 0,49 & 0,02 & 0,05 \\
& $\underline{\text { Insgesamt }}$ & $\underline{1.587}$ & $\underline{0,94}$ & $\underline{0,43}$ & $\underline{0,01}$ & $\underline{0,03}$ \\
\multirow{3}{*}{ Insgesamt } & kons. & 1.794 & 0,88 & 0,28 & 0,01 & 0,02 \\
& konv. & 2.061 & 0,84 & 0,37 & 0,01 & 0,02 \\
& $\underline{\text { Insgesamt }}$ & $\underline{\underline{3.855}}$ & $\underline{0,86}$ & $\underline{0,33}$ & $\underline{0,01}$ & $\underline{0,01}$ \\
\hline
\end{tabular}

Der Standort SH wies mit durchschnittlichen hEW von 0,94 $\pm 0,43 \mathrm{MPa}$ sowohl die höheren Messwerte als auch die größere Streuung gegenüber NRW $(0,80 \pm 0,22 \mathrm{MPa})$ auf. Auch die Bodenbearbeitung hatte einen signifikanten Einfluss, der an den Versuchsstandorten jedoch unterschiedlich ausgeprägt war: während auf $\mathrm{SH}_{\text {konv }}$ ein um $+0,11 \mathrm{MPa}$ höherer $\mathrm{hEW}$ gegenüber $\mathrm{SH}_{\text {kons }}$ ermittelt wurde, konnte am Standort NRW bei wendender Bodenbearbeitung ein um -0,12 MPa niedrigerer hEW gemessen werden. Zwischen den konservierend bewirtschafteten Flächen $\mathrm{NRW}_{\text {kons }}(0,87 \pm 0,19 \mathrm{MPa})$ und $\mathrm{SH}_{\text {kons }}$ $(0,89 \pm 0,36 \mathrm{MPa})$ bestanden keine signifikanten Unterschiede, wohl aber zwischen den Flächen konventioneller Bewirtschaftung: auf $N_{R} W_{\text {konv }}$ wurden mit $0,75 \pm 0,23 \mathrm{MPa}$ die niedrigsten und auf $\mathrm{SH}_{\text {konv }}$ die höchsten hEW $(1,00 \pm 0,49 \mathrm{MPa})$ festgestellt (vgl. Tabelle 6).

Über den Versuchszeitraum betrachtet wurden in 2006 die höchsten hEW gemessen, wobei die Fläche $\mathrm{SH}_{\text {konv }}$ einen starken Einfluss hatte, da sie mit $1,22 \pm 0,58 \mathrm{MPa}$ um $+46,1 \%$ höhere Messwerte als alle übrigen Versuchsflächen lieferte. Die geringsten hEW wurden im Frühjahr 2008 mit durchschnittlich 0,78 $\pm 0,26 \mathrm{MPa}$ ermittelt (vgl. Tabelle A 21).

In Abbildung 23 ist dargestellt, wie sich der hEW über die Messtiefenbereiche der einzelnen Flächen im Mittel der Untersuchungen verändert. Es ist zu 
erkennen, dass sich auch in den TASIS-Messwerten die charakteristischen bewirtschaftungsbedingten Verläufe des Eindringwiderstandes über die Messtiefe wieder finden, wie sie bei den VP-Messungen beschrieben wurden.

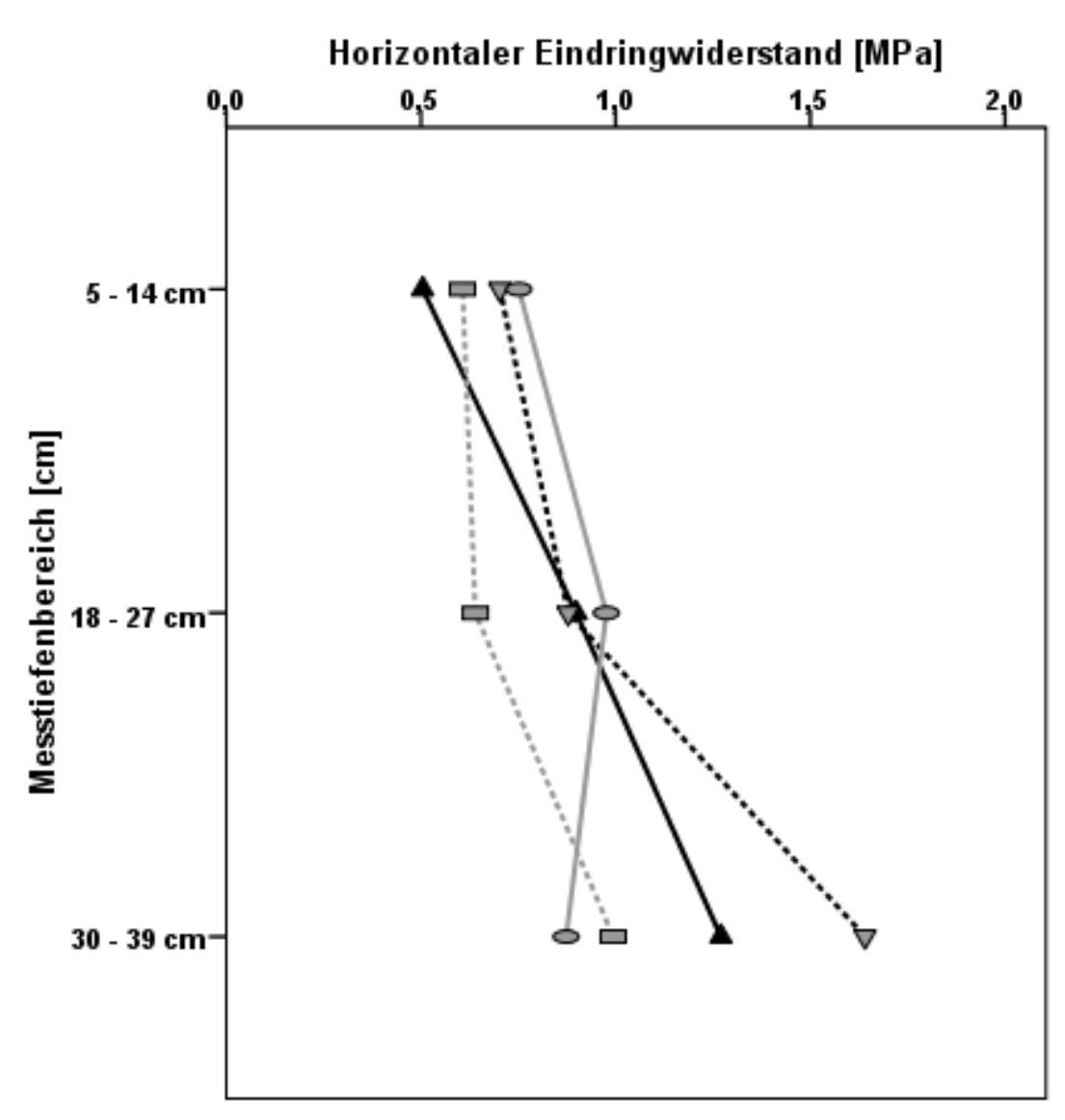

Fläche

$$
\begin{aligned}
& - \text { SH kons. } \\
& \hline \ldots \text { SH konv. } \\
& \hline \text { IRW kons. } \\
& \ldots \text { NRW konv. } \\
& \text { A SH kons. } \\
& \nabla \text { SH konv. } \\
& 0 \text { NRW kons. } \\
& \square \text { NRW konv. }
\end{aligned}
$$

Abbildung 23: Horizontale Eindringwiderstände [MPa] nach Flächen und Messtiefen ( $\mathrm{n}=3.855, \mathrm{GD}_{5 \%} \approx 0,03 \mathrm{MPa}, \bar{x}$ über 5 Termine, 6 Varianten, 2 Blöcke, 6 Wdh.).

Eine analoge Darstellung der Daten der vEW findet sich in Abbildung 24: 


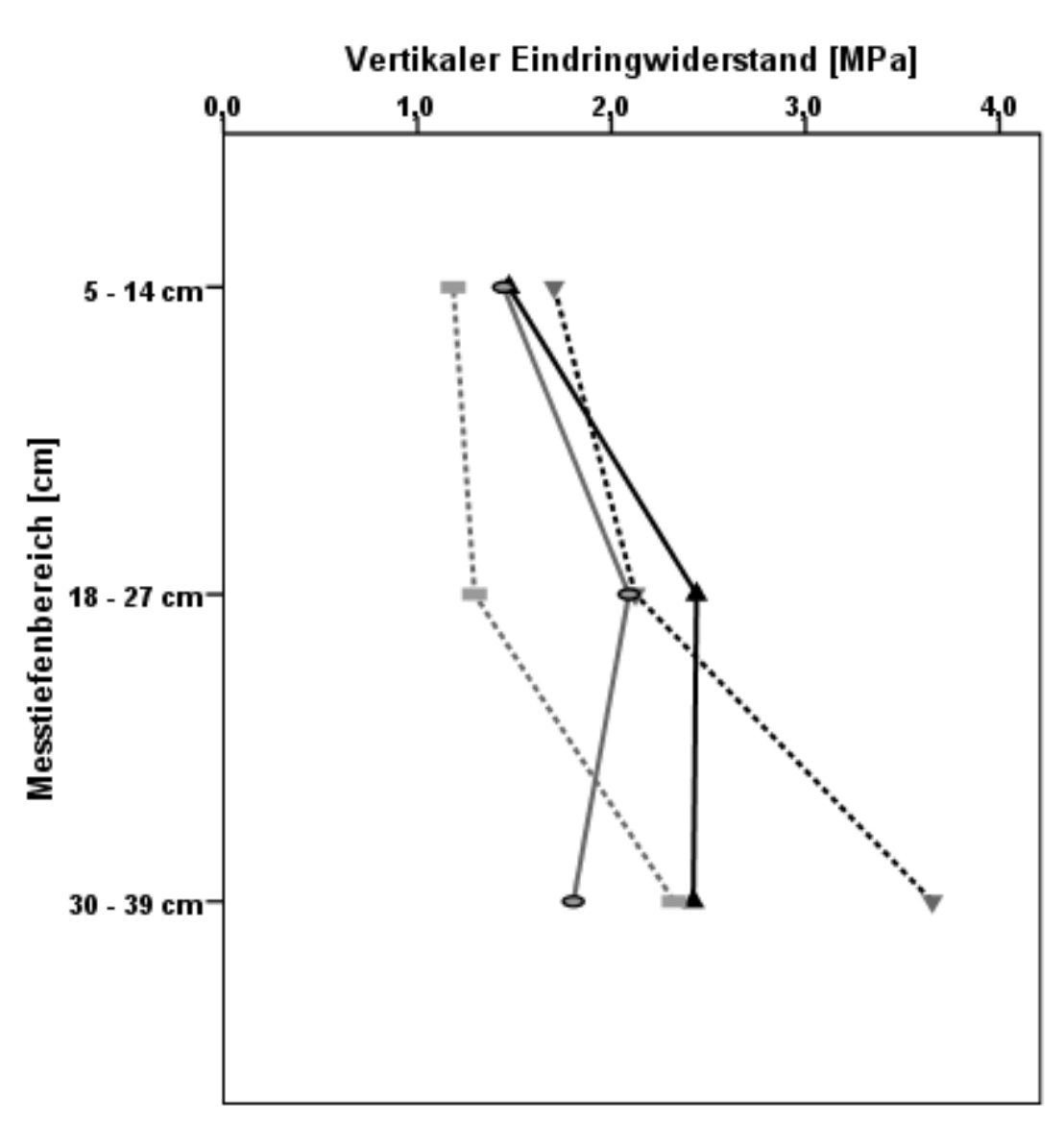

Fläche

- SH kons.

.... SH konv.

- IRW kons.

.... IRW konv.

A SH kons.

$\nabla$ SH konv.

- IIRW kons.

= URW konv.

Abbildung 24: Vertikale Eindringwiderstände [MPa] nach erfolgter Befahrung nach Flächen und Messtiefen $\left(n=5.710, G_{5 \%} \approx 0,12 \mathrm{MPa}, \overline{\mathrm{x}}\right.$ über 5 Termine, 6 Varianten, 2 Blöcke, 6 Wdh.).

Die konventionell bewirtschafteten Flächen weisen locker gelagerte Bodenschichten bis $27 \mathrm{~cm}$ sowie nachfolgend ansteigende Messwerte im Bereich der Pflugsohle auf, wobei sich die schleswig-holsteinische Versuchsfläche durch insgesamt höhere hEW auszeichnet. Bei den konservierend bewirtschafteten Flächen hingegen kann ein gleichförmiger Verlauf der Eindringwiderstände über die Messtiefen nicht beobachtet werden: während auf $\mathrm{NRW}_{\text {kons }}$ die Werte bis $18-27 \mathrm{~cm}$ steigen, um danach wieder auf ein niedrigeres Niveau abzufallen, erhöhen sich die hEW auf $\mathrm{SH}_{\text {kons }}$ auch in dieser Tiefe weiter, bleiben aber unterhalb der Werte von $\mathrm{SH}_{\text {konv. }}$. 


\subsubsection{Horizontale Eindringwiderstände nach Flächen}

Nachfolgend werden die Daten der einzelnen Versuchsflächen untersucht. Daran schließt sich eine Analyse des Einflusses der einzelnen Faktoren an.

\section{Schleswig-Holstein, konservierende Bodenbewirtschaftung}

Auf $\mathrm{SH}_{\text {kons }}$ weist keine der befahrenen Parzellen einen niedrigeren $\mathrm{hEW}$ auf als die Kontrolle $(0,87 \pm 0,37 \mathrm{MPa})$. Allerdings können für die höheren hEW der einzelnen Varianten die Unterschiede auch nicht als statistisch signifikant abgesichert werden, da sie sich um maximal 0,05 MPa $(7,5 \mathrm{Mg} @ 250 \mathrm{kPa})$ von der Kontrolle unterscheiden.

In 2006 zeigten sich auf $\mathrm{SH}_{\text {kons }}$ geringfügige Unterschiede zwischen den einzelnen Belastungsvarianten. Lediglich die Variante 6,3Mg@160kPa wies in den zwei oberen Messtiefenbereichen signifikant höhere hEW gegenüber der Kontrolle auf. Das Niveau der gemessenen hEW nahm mit der Messtiefe zu, ebenso die Streuung der Messwerte (vgl. Tabelle A 26).

Durch die Optimierung von TASIS konnten in 2007 im Bereich $5-14 \mathrm{~cm}$ bereits differenzierte Messwerte gewonnen werden, die in den oberen Messtiefen auch breit gestreut waren und mit durchschnittlich 0,56 $\pm 0,14 \mathrm{MPa}$ um $+44 \%$ höher als in 2006 lagen. Tendenziell wiesen die befahrenen Bereiche leicht erhöhte hEW gegenüber der Kontrolle aus. In den Messtiefenbereichen $18-27 \mathrm{~cm}$ und $30-39 \mathrm{~cm}$ ließ sich auch ein leichter Anstieg mit steigender mechanischer Belastung erkennen. Eine Ausnahme stellte die Variante 7,5Mg@350kPa dar hier lag der durchschnittliche hEW in 2007 in beiden Messtiefenbereichen leicht unterhalb der Kontrolle. Die Unterschiede waren jedoch für alle Varianten mit $<0,05 \mathrm{MPa}$ als statistisch nicht signifikant anzusehen (vgl. Tabelle A 27). 


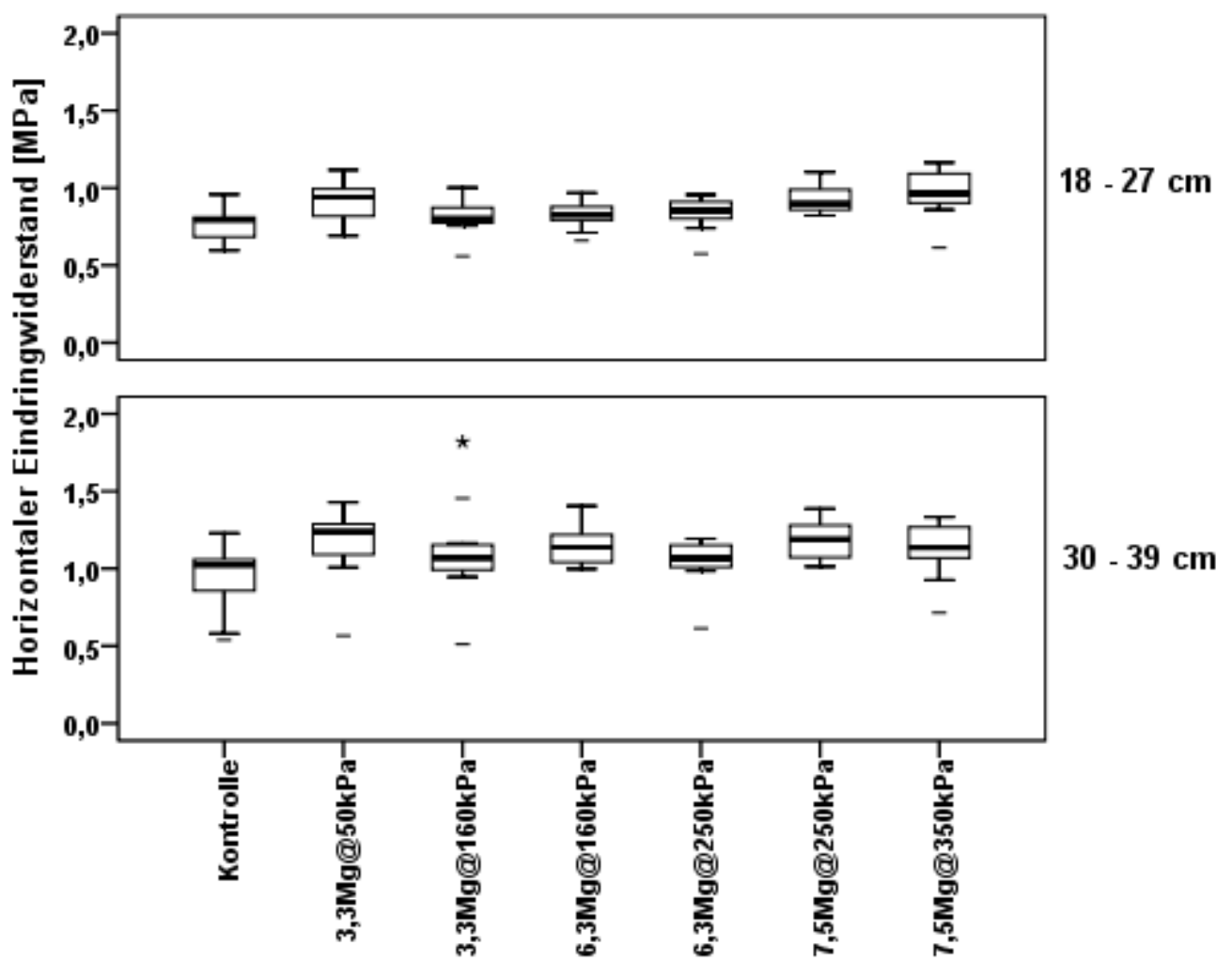

Abbildung 25: Horizontale Eindringwiderstände [MPa] der Fläche $\mathrm{SH}_{\text {kons }}$ im Jahr 2008 in den Messtiefenbereichen $18-27 \mathrm{~cm}$ und $30-39 \mathrm{~cm}$ nach Belastungsvariante $(\mathrm{n}=87$, $\mathrm{GD}_{5 \%} \approx 0,09 \mathrm{MPa}$, $\bar{x}$ über 2 Blöcke, $6 \mathrm{Wdh}$.).

Das Jahr 2008 lieferte von allen Messterminen die differenziertesten Daten. In dieser Kampagne wies die Kontrolle mit 0,73 $\pm 0,23 \mathrm{MPa}$ die durchschnittlich niedrigsten $\mathrm{hEW}$ von allen Varianten auf. Die befahrenen Parzellen lassen mit steigender Belastung auch steigende hEW erkennen. Signifikant höhere hEW im Mittel der Messtiefen sind nur bei der Variante 3,3Mg@50kPa und der Varianten mit einer Radlast von 7,5 Mg festzustellen, was insbesondere auf den Ergebnissen aus dem mittleren und unteren Messtiefenbereich beruht (vgl. Abbildung 25).

Neben den genannten Varianten ist auch bei $6,3 \mathrm{Mg} @ 160 \mathrm{kPa}$ in den Messtiefenbereichen $18-27 \mathrm{~cm}$ und $30-39 \mathrm{~cm}$ ein signifikant höherer Wert des hEW gegenüber der Kontrolle festzustellen. Die Anstiege in den hEW sind jedoch nicht stetig: bei Radlasten von $3,3 \mathrm{Mg}$ und $6,3 \mathrm{Mg}$ weisen jeweils die höheren RID die niedrigeren hEW auf, wobei die Unterschiede maximal $0,11 \mathrm{MPa}$ betragen. 


\section{Schleswig-Holstein, konventionelle Bodenbewirtschaftung}

Auch auf der konventionell bewirtschafteten Fläche des schleswigholsteinischen Versuchsstandortes konnten in 2006 in den Bodentiefen 5 $14 \mathrm{~cm}$ und $30-39 \mathrm{~cm}$ keine signifikanten Unterschiede der befahrenen Parzellen gegenüber der Kontrolle ermittelt werden, die in diesen Messtiefen durchschnittliche hEW aufwies. Die höchsten Messwerte konnten jeweils für Varianten mit 7,5 Mg Radlast gemessen werden (vgl. Tabelle A 29). In 18 $27 \mathrm{~cm}$ Tiefe war zu beobachten, dass mit Ausnahme von 7,5Mg@350kPa die hEW aller Varianten signifikant unterhalb der Kontrolle lagen, die mit $1,09 \pm 0,21 \mathrm{MPa}$ die höchsten Messwerte in diesem Bereich auswies. Die Differenzen der signifikanten Varianten gegenüber der Kontrolle betrugen zwischen -0,19 MPa (7,5Mg@250kPa) und -0,27 MPa (3,3Mg@50kPa). Die belasteten Bereiche wiesen mit steigender Radlast auch leicht steigende hEW auf (vgl. Abbildung 26). Bei 3,3 Mg Radlast betrug der hEW 0,86 MPa, bei $6,3 \mathrm{Mg}$ lag er bei $0,89 \mathrm{MPa}$, und auf den mit $7,5 \mathrm{Mg}$ belasteten Bereichen konnte ein $\mathrm{hEW}$ von $0,94 \mathrm{MPa}$ ermittelt werden.

In 2007 musste aufgrund des starken Bewuchses durch aufgelaufenes Ausfallgetreide auf der Fläche auf eine Datenaufnahme in $30-39 \mathrm{~cm}$ verzichtet werden, um durch ein Verstopfen des TASIS nicht die Qualität der Datenaufnahme aller Messtiefenbereiche zu gefährden. In $5-14 \mathrm{~cm}$ Messtiefe lagen die hEW der befahrenen Bereiche durchschnittlich $+11,5 \%$ über den hEW der Kontrolle. Im Bereich 18-27 cm betrug dieser Unterschied nur 3,6\%. Die höchsten hEW wiesen in dieser Bodentiefe die Varianten mit 6,3 Mg und 7,5 Mg Radlast auf. Der Gesamtdurchschnitt aller Messdaten wich in 2007 auf $\mathrm{SH}_{\text {konv }}$ um +0,05 MPa vom Durchschnitt der Kontrollparzellen $(0,85 \pm 0,22 \mathrm{MPa})$ ab (vgl. Tabelle A 30). 


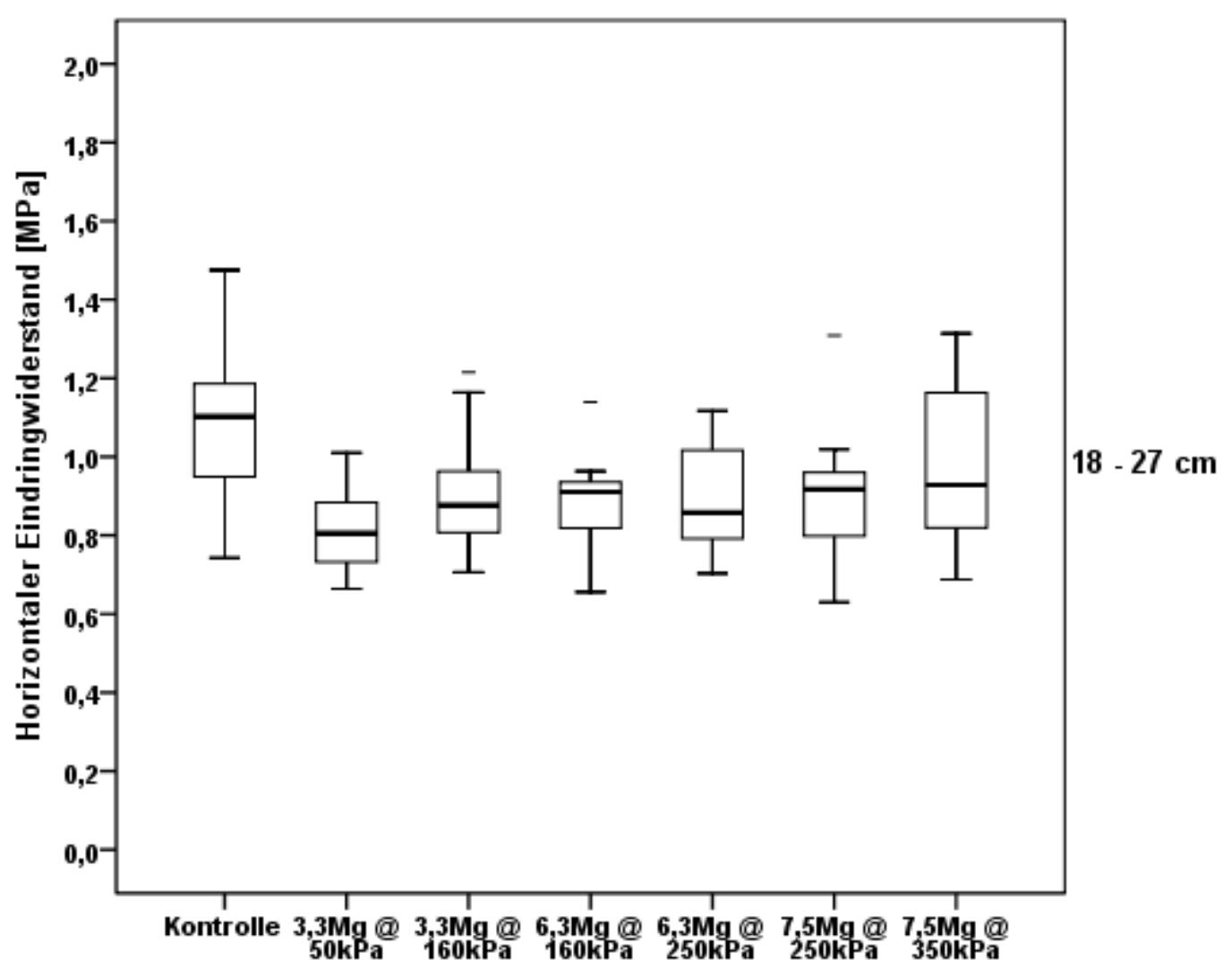

Abbildung 26: Horizontale Eindringwiderstände [MPa] gemessen auf $\mathrm{SH}_{\text {konv }}$ im Herbst $2006 \mathrm{im}$ Messtiefenbereich $18-27 \mathrm{~cm}\left(\mathrm{n}=95, \mathrm{GD}_{5 \%} \approx 0,12 \mathrm{MPa}, \overline{\mathrm{x}}\right.$ über 2 Blöcke, 6 Wdh.).

Im Jahr 2008 betrugen die hEW im Bereich $5-14 \mathrm{~cm}$ durchschnittlich 0,51 MPa und lagen damit unterhalb der Werte von 2007 (0,77 MPa) sowie 2006 $(0,83 \mathrm{MPa})$. Auch in den anderen Messtiefenbereichen wurden in 2008 die niedrigsten $\mathrm{hEW}$ ermittelt.

In $5-14 \mathrm{~cm}$ wies die Kontrolle die niedrigsten Messwerte $(0,49 \pm 0,12 \mathrm{MPa})$ gegenüber den befahrenen Bereichen aus, deren hEW $+12,7 \%$ höher lagen. Im Bereich $18-27 \mathrm{~cm}$ betrug die Differenz der befahrenen gegenüber den unbefahrenen Bereichen durchschnittlich $+9,2 \%$. Darüber hinaus konnte der hEW der Varianten 6,3Mg@250kPa $(0,71 \pm 0,09 \mathrm{MPa})$ und 7,5Mg@350kPa $(0,78 \pm 0,13 \mathrm{MPa})$ als signifikant höher gegenüber der Kontrolle $(0,62 \pm 0,16 \mathrm{MPa})$ abgesichert werden (vgl. Abbildung 27). Die übrigen Messwerte in diesem Tiefenbereich unterschieden sich nur geringfügig unter- 
einander, und Signifikanzen gegenüber der Kontrolle waren nicht zu ermitteln (vgl. Tabelle A 30).

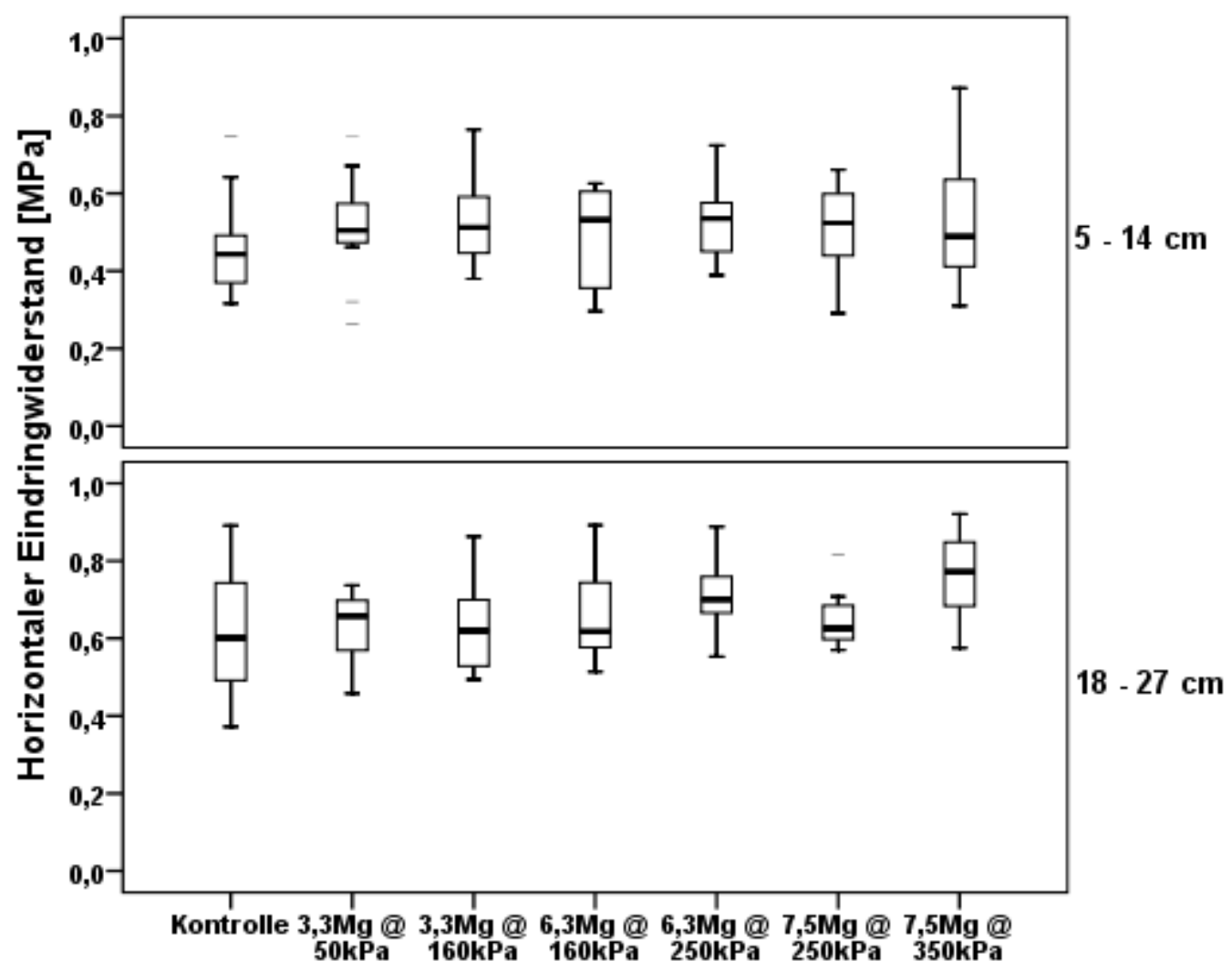

Abbildung 27: Horizontale Eindringwiderstände [MPa] gemessen auf $\mathrm{SH}_{\text {konv }}$ im Frühjahr 2008 in den Messtiefenbereichen $5-14 \mathrm{~cm}$ und $18-27 \mathrm{~cm}\left(\mathrm{n}=192, \mathrm{GD}_{5 \%} \approx 0,09 \mathrm{MPa}, \overline{\mathrm{x}}\right.$ über 2 Blöcke, 6 Wdh.).

Im Messtiefenbereich $30-39 \mathrm{~cm}$ sind nur bei $3,3 \mathrm{Mg} @ 50 \mathrm{kPa}$ und $7,5 \mathrm{Mg} @ 350 \mathrm{kPa}$ leicht erhöhte hEW von durchschnittlich +0,11 MPa zu beobachten, während für die übrigen Varianten um den selben Betrag niedrigere hEW gemessen wurden. Signifikanzen konnten nicht ermittelt werden.

\section{Nordrhein-Westfalen, konservierende Bodenbewirtschaftung}

Auf der Versuchfläche $\mathrm{NRW}_{\text {kons }}$ zeigten die $\mathrm{hEW}$ in 2006 keine signifikanten Anstiege in Abhängigkeit von der mechanischen Belastung. In den Messtiefenbereichen $18-27 \mathrm{~cm}$ und $30-39 \mathrm{~cm}$ wiesen die Kontrollparzellen 
die jeweils höchsten hEW auf. Im untersten Messbereich lagen die hEW aller Belastungsvarianten mit Ausnahme von 7,5Mg@250kPa und 7,5Mg@350kPa signifikant unterhalb des Wertes der Kontrolle. Die Unterschiede innerhalb der Lastvarianten waren dabei insgesamt gering und statistisch nicht signifikant (vgl. Tabelle A 32).

In 2007 waren in der Messtiefe $5-14 \mathrm{~cm}$ für die Varianten mit $160 \mathrm{kPa}$ Reifeninnendruck $(0,80 \pm 0,08 \mathrm{MPa}$ bei $3,3 \mathrm{Mg}$ Radlast bzw. 0,81 $\pm 0,09 \mathrm{MPa}$ bei $6,3 \mathrm{Mg}$ Radlast) signifikant höhere $\mathrm{hEW}$ gegenüber der Kontrolle $(0,71 \pm 0,13 \mathrm{MPa})$ festzustellen. Im Durchschnitt lagen die hEW der befahrenen Parzellen in diesem Messtiefenbereich um $+10,9 \%$ höher gegenüber der Kontrolle, während die Werte in den darunter liegenden Messtiefen nur geringfügig um den Messwert der Kontrolle schwankten (vgl. Tabelle A 33).

Wie bereits in 2006 so war auch im Frühjahr 2008 zu beobachten, dass im Bereich $30-39 \mathrm{~cm}$ die höchsten hEW in den Kontrollparzellen ermittelt wurden und die Varianten 3,3Mg@160kPa und 7,5Mg@350kPa sowie jene Varianten mit $6,3 \mathrm{Mg}$ Radlast signifikant niedrigere Messwerte aufwiesen. Insbesondere die Kontrollparzellen im mittleren Teil der Versuchsanlage zeichneten sich durch hohe Messwerte aus. In den Messtiefen 5-14 cm und $18-27 \mathrm{~cm}$ der befahrenen Bereiche lagen die hEW (mit jeweils einer Ausnahme) stets über der Kontrolle. Doch auch hier war eine stetige Zunahme des Messwertes mit steigender mechanischer Belastung nicht klar zu erkennen.

Über den gesamten Versuchszeitraum konnten in der Messtiefe $5-14 \mathrm{~cm}$ in allen Jahren erhöhte hEW der befahrenen Parzellen gegenüber den Kontrollparzellen festgestellt werden. Die Differenzen zwischen hEW und Kontrolle betrugen jeweils $+1,8 \%$ (2006), $+10,9 \%$ (2007) und $+5,6 \%$ (2008). Die Werte in 18 - $27 \mathrm{~cm}$ blieben hingegen nahezu unverändert. Sie bewegten sich auf einem Niveau entweder leicht unterhalb der Kontrolle $(-4,0 \%$ in 2006$)$ oder leicht darüber $(+0,2 \%$ in $2007,+2,9 \%$ in 2008). In der Messtiefe $30-39 \mathrm{~cm}$ lagen die Messwerte der befahrenen Parzellen unterhalb jener der Kontrolle. Die Differenz in 2007 betrug $-2,2 \%$, in den Jahren 2006 und 2008 fiel sie mit $-11,3 \%$ bzw. $-11,1 \%$ noch deutlicher aus. 


\section{Nordrhein-Westfalen, konventionelle Bodenbewirtschaftung}

Auf NRW konv waren in den Jahren 2006 und 2007 in der Messtiefe 5 - $14 \mathrm{~cm}$ nur geringe Unterschiede zwischen den belasteten Parzellen und der Kontrolle festzustellen, von denen keiner statistisch signifikant war. Die Mittelwerte der einzelnen Varianten wiesen 2006 mit einer Standardabweichung von $\pm 0,05 \mathrm{MPa}$ die geringste Streuung aller Messungen auf. In 2007 betrug sie $\pm 0,08 \mathrm{MPa}$.

In der Messtiefe $18-27 \mathrm{~cm}$ konnten in 2006 für alle Belastungsvarianten ab 3,3 Mg Radlast und einem Reifeninnendruck von $160 \mathrm{kPa}$ signifikant höhere hEW gegenüber der Kontrolle $(0,57 \pm 0,07 \mathrm{MPa})$ gemessen werden. Die Messwerte lassen im Trend eine Zunahme der hEW mit steigender mechanischer Belastung erkennen. Bei $7,5 \mathrm{Mg} @ 350 \mathrm{kPa}$ waren mit $0,71 \pm 0,08 \mathrm{MPa}$ die höchsten Messwerte festzustellen. Die hEW der Varianten $3,3 \mathrm{Mg} @ 50 \mathrm{kPa}$ und nur Schlepper hingegen lagen mit 0,58 \pm 0,06 MPa bzw. $0,60 \pm 0,08 \mathrm{MPa}$ zwar leicht über jenen der Kontrolle, unterschieden sich von dieser aber nicht signifikant.

In der Bodenschicht $30-39 \mathrm{~cm}$ streuten die Werte mit einer durchschnittlichen Standardabweichung von $\pm 0,30 \mathrm{MPa}$ deutlich stärker als in den darüber liegenden Messbereichen. Der Mittelwert aller Varianten liegt in dieser Messtiefe bei $0,98 \mathrm{MPa}$ und trennt Varianten mit geringer mechanischer Belastung (Kontrolle, nur Schlepper und jene mit 3,3 Mg Radlast), deren hEW sich einheitlich unterhalb dieses Wertes befinden, von Varianten mit Radlast $>6,3 \mathrm{Mg}$, die hEW oberhalb dieses Wertes aufweisen. Statistisch signifikante Unterschiede waren hierbei nicht zu ermitteln (vgl. Tabelle A 35).

Im darauf folgenden Jahr 2007 konnten bei Varianten der Radlast 7,5 Mg sowie bei den Varianten 3,3Mg@50kPa und 6,3Mg@160kPa sowohl in 30 - $39 \mathrm{~cm}$ als auch in $18-27 \mathrm{~cm}$ signifikant höhere hEW gegenüber der Kontrolle beobachtet werden (vgl. Abbildung 28). In beiden Messtiefenbereichen wurden für die Kontrollparzellen mit 0,98 $\pm 0,14 \mathrm{MPa}(30-39)$ bzw. 0,62 $\pm 0,05 \mathrm{MPa}$ (18 $27 \mathrm{~cm}$ ) die niedrigsten Durchschnittswerte ermittelt. Demgegenüber betrugen die durchschnittlichen hEW der befahrenen Parzellen in diesen Bereichen
$1,11 \pm 0,20 \mathrm{MPa}$
$(30-39 \mathrm{~cm})$
bzw.
$0,67 \pm 0,07 \mathrm{MPa}$
$(18-27 \mathrm{~cm}) . \quad \mathrm{Im}$ 
Messtiefenbereich 5-14 cm lag die Spannweite der Messwerte bei 0,07 MPa bei einer Standardabweichung von $\pm 0,08 \mathrm{MPa}$. Signifikante Unterschiede konnten nicht ermittelt werden (vgl. Tabelle A 36).

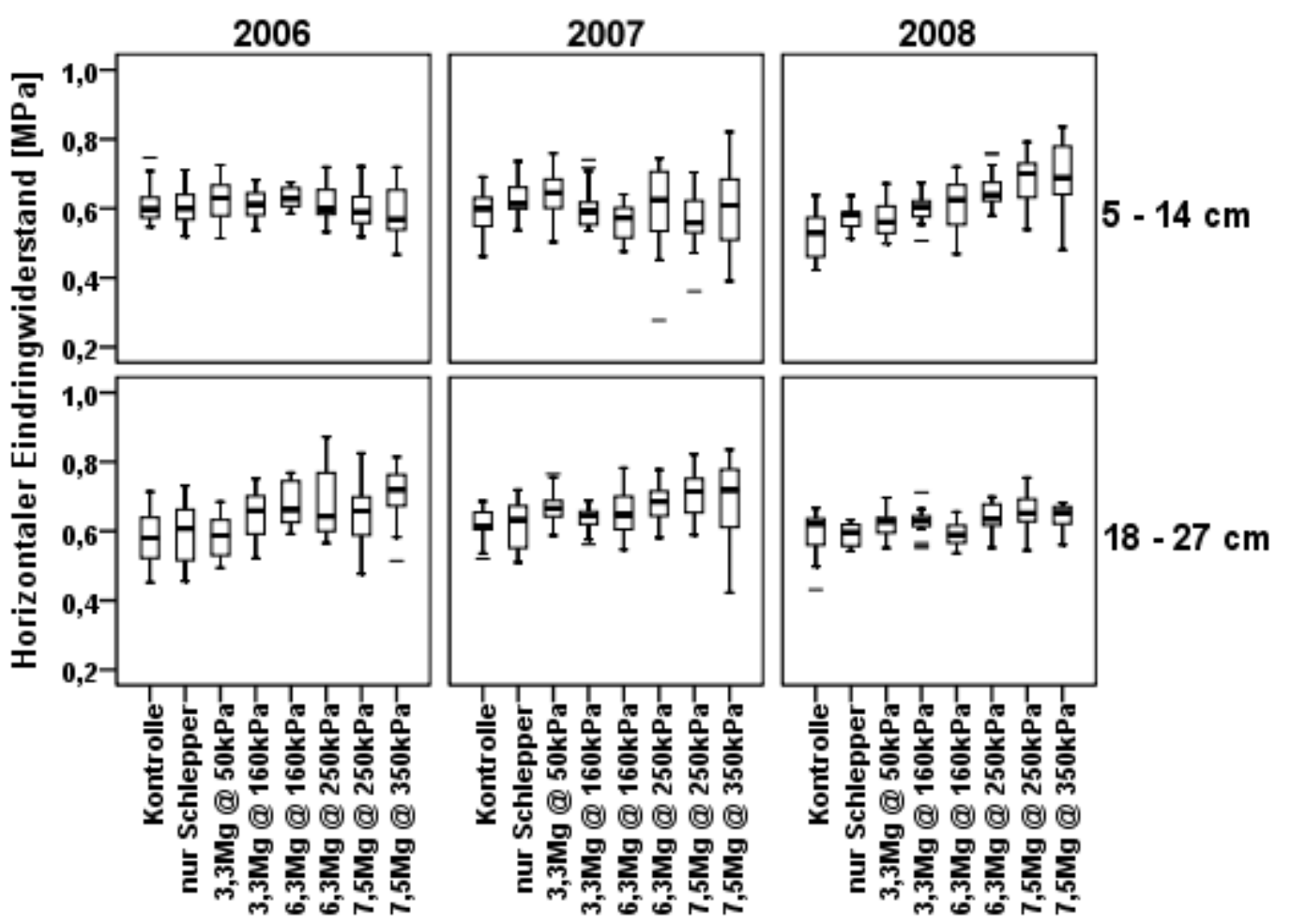

Abbildung 28: Horizontale Eindringwiderstände [MPa] der Fläche $N_{R W} W_{\text {konv }}$ in den Messtiefenbereichen $5-14 \mathrm{~cm}$ und $18-29 \mathrm{~cm}$ nach Jahr und Belastungsvariante ( $\mathrm{n}=864, \mathrm{GD}_{5 \%} \approx 0,04 \mathrm{MPa}, \overline{\mathrm{x}}$ über 3 Blöcke, $6 \mathrm{Wdh}$.).

Die Daten aus 2008 lassen am deutlichsten einen Zusammenhang zwischen mechanischer Belastung und hEW erkennen: während in den Messtiefen 5 $14 \mathrm{~cm}$ und $18-27 \mathrm{~cm}$ ein Ansteigen des hEW mit zunehmender mechanischer Belastung zu beobachten ist ( $\mathrm{vgl}$. Abbildung 28), zeichnet sich in der Messtiefe $30-39 \mathrm{~cm}$ ein Absinken des hEW mit steigenden Radlasten und Reifeninnendrücken ab (vgl. Abbildung 29). Im Oberboden wies die Kontrolle mit 0,52 $\pm 0,06 \mathrm{MPa}$ den geringsten hEW aus, dessen Werte sich in den belasteten Parzellen zwischen 0,57 $\pm 0,06 \mathrm{MPa}$ (Variante nur Schlepper) und 
$0,69 \pm 0,10 \mathrm{MPa}(7,5 \mathrm{Mg} @ 350 \mathrm{kPa})$ bewegten. Alle Unterschiede gegenüber der unbefahrenen Kontrolle waren statistisch signifikant.
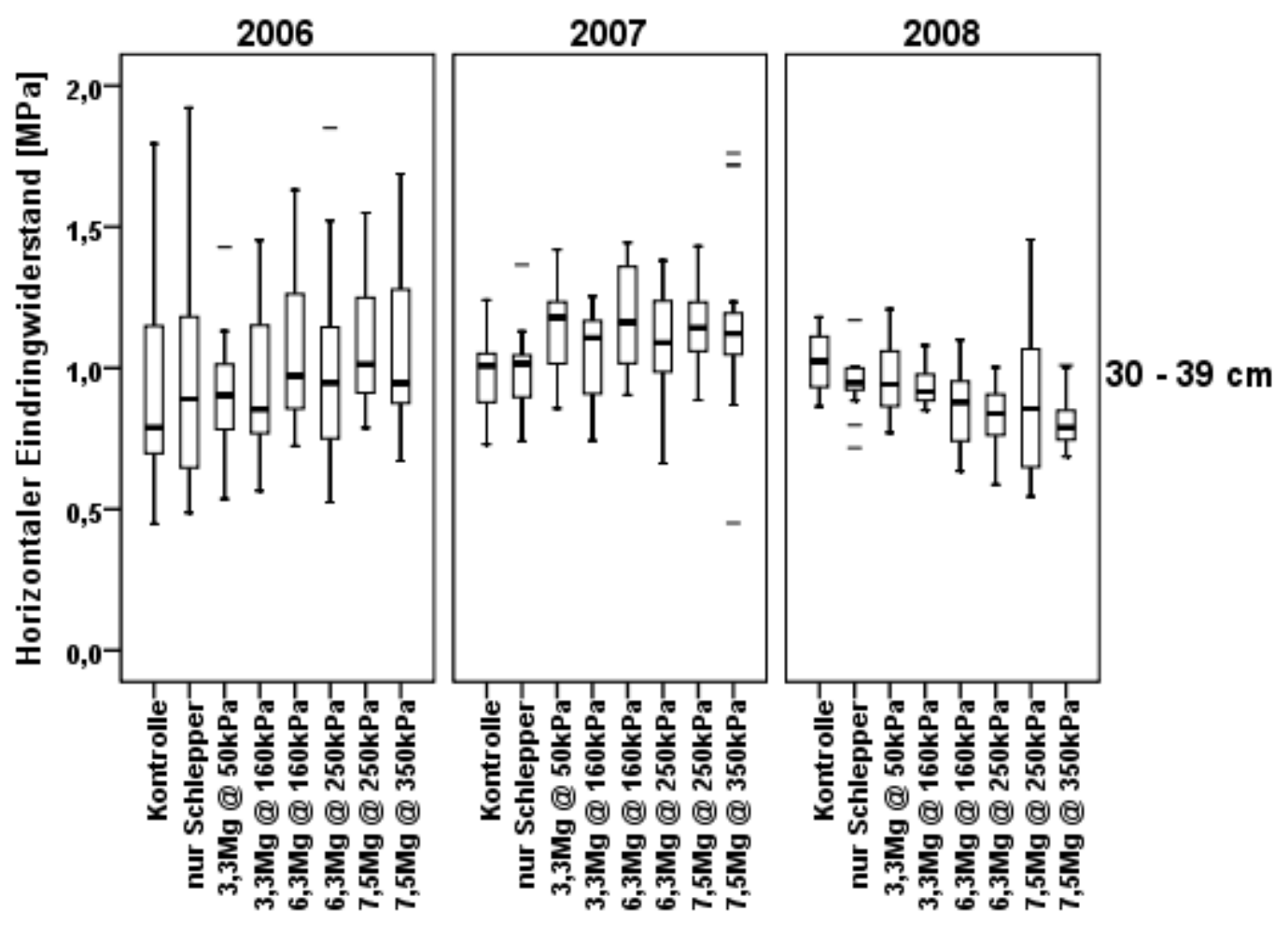

Abbildung 29: Horizontale Eindringwiderstände [MPa] der Fläche $\mathrm{NRW}_{\text {konv }}$ im Messtiefenbereichen $30-39 \mathrm{~cm}$ nach Jahr und Belastungsvariante ( $n=432$, $\mathrm{GD}_{5 \%} \approx 0,12 \mathrm{MPa}, \overline{\mathrm{x}}$ über 3 Blöcke, $6 \mathrm{Wdh}$.).

In 18-27 cm war die Stetigkeit im Anstieg des hEW bei zunehmender mechanischer Belastung weniger deutlich ausgeprägt: während Kontrolle, nur Schlepper und 6,3Mg@160kPa mit Werten < 0,6 MPa den unteren Bereich des Datenspektrums darstellten, lagen 3,3Mg@50kPa und 3,3Mg@160kPa mit Werten von 0,62 MPa bzw. 0,63 MPa geringfügig darüber. Alle Varianten mit einer Radlast von $6,3 \mathrm{Mg}$ und mindestens $250 \mathrm{kPa}$ RID wiesen mit Werten zwischen 0,64 und 0,65 MP einen signifikant höheren hEW gegenüber der Kontrolle aus.

Während in den oberen Messtiefen ein Anstieg der Messwerte bei mechanischer Belastung $\mathrm{zu}$ beobachten ist, kann in Tiefe $30-39 \mathrm{~cm}$ ein 
Absinken der hEW bei belastungsstarken Varianten festgestellt werden. Den höchsten Messwert in dieser Tiefe weist mit 1,02 $\pm 0,10 \mathrm{MPa}$ die Kontrolle aus, gefolgt von den Varianten mit 3,3 Mg Radlast und nur Schlepper. Den niedrigsten Messwert wurde bei 7,5Mg@350kPa mit 0,81 $\pm 0,10 \mathrm{MPa}$ ermittelt. Die hEW der Varianten mit einer Radlast $\geq 6,3 \mathrm{Mg}$ unterschieden sich hierbei signifikant von der Kontrolle (vgl. Tabelle A 37).

Signifikante Zunahmen der hEW der befahrenen Bereiche gegenüber der Kontrolle konnten auf $\mathrm{NRW}_{\text {konv }}$ in allen Messtiefenbereichen und zu allen Messzeitpunkten festgestellt werden. Besonders ausgeprägt waren sie in 2006 in $18-27 \mathrm{~cm} \mathrm{(+13,0 \% ),} \mathrm{in} 2007$ in $30-39 \mathrm{~cm}(+13,4 \%)$ und in 2008 in $5-14 \mathrm{~cm}$ $(+20,3 \%)$. Erkennbar ist darüber hinaus der Trend, das hEW mit steigender mechanischer Belastung zunehmen. Eine Ausnahme stellen die Messergebnisse des Messtiefenbereiches $30-39 \mathrm{~cm}$ dar: hier konnten mit steigender Radlast kontinuierlich sinkende hEW ermittelt werden.

\subsubsection{Effekte der Hauptfaktoren Radlast und Reifeninnendruck auf den horizontalen Eindringwiderstand}

Analog zur Analyse der VP-Daten wurden auch die TASIS-Daten auf den Einfluss der Fahrwerksparameter Radlast und Reifeninnendruck untersucht.

Bei einer Veränderung der Radlast von 3,3 Mg auf 6,3 Mg erhöht sich der hEW bei einem konstantem RID von $160 \mathrm{kPa}$ im Mittel der Untersuchungen um $0,02 \mathrm{MPa}$. Die Zunahme des hEW infolge höherer mechanischer Belastung ist im Jahr 2006 mit 0,05 MPa am deutlichsten ausgeprägt, jedoch nicht statistisch signifikant. Auch bei der Betrachtung der einzelnen Flächen sind keine Signifikanzen zwischen den hEW bei verschiedenen Radlasten festzustellen. Es können aber tendenziell höhere hEW bei höherer Radlast beobachtet werden. Die stärkste Veränderung wurde auf $\mathrm{SH}_{\text {kons }}$ mit $+0,04 \mathrm{MPa}$ ermittelt. Insgesamt zeichnen sich die konservierend bewirtschafteten Flächen durch geringfügig stärkere Zunahmen der hEW $(+0,03 \mathrm{MPa})$ aus als die konventionellen Standorte $(+0,01 \mathrm{MPa})$. Bei konservierender Bodenbearbeitung fällt der Anstieg mit +0,05 MPa im Bereich $18-27 \mathrm{~cm}$ am deutlichsten aus, 
während unter Pflug die größte Erhöhung des hEW mit +0,03 MPa in der Messtiefe 18 - $39 \mathrm{~cm}$ festzustellen ist. Keine der Differenzen kann als statistisch signifikant abgesichert werden. Im Jahr 2008 wurden auf $\mathrm{NRW}_{\text {konv }}$ in $18-27 \mathrm{~cm}$ $(-0,03 \mathrm{MPa})$ und $30-39 \mathrm{~cm}(-0,08 \mathrm{MPa})$ abnehmende hEW mit steigender Radlast gemessen (vgl. Tabelle A 40).

Auch bei einer Radlasterhöhung von $6,3 \mathrm{Mg}$ auf $7,5 \mathrm{Mg}$ bei $250 \mathrm{kPa}$ RID können erhöhte hEW beobachtet werden, die durchschnittlich +0,02 MPa betragen. Bei weitergehender Betrachtung finden sich die deutlichsten Unterschiede im Jahr 2008 (+0,03 MPa) sowie bei konservierender Bodenbearbeitung $(+0,03 \mathrm{MPa})$. Bei nicht-wendender Bodenbearbeitung nimmt der hEW gleichmäßig über die Messtiefenbereiche zu, während bei wendender Bodenbearbeitung der stärkste Anstieg $(+0,04 \mathrm{MPa})$ im Bereich $30-39 \mathrm{~cm}$ stattfindet (vgl. Tabelle A 41).

Bei der Betrachtung des Einflusses des RID auf den hEW bei 3,3 Mg Radlast ist festzustellen, dass eine Erhöhung von $50 \mathrm{kPa}$ auf $160 \mathrm{kPa}$ zu einer geringfügigen Abnahme des hEW von $-0,02 \mathrm{MPa}$ führt (vgl. Tabelle A 42). Dieses ist über alle Messkampagnen zu beobachten, ebenso auf allen Flächen und in allen Messtiefenbereichen. Die deutlichsten Abnahmen entfallen auf das Jahr $2008(-0,04 \mathrm{MPa}), \mathrm{SH}_{\text {kons }}(-0,04 \mathrm{MPa})$ und den Messtiefenbereich 30 $39 \mathrm{~cm}(-0,06 \mathrm{MPa})$.

Die Erhöhung des RID bei 6,3 Mg Radlast bewirkt nahezu keinen Effekt auf die hEW: die Werte beider Varianten unterscheiden sich im Mittel nicht, und Unterschiede zwischen den Terminen und Messtiefenbereichen betragen maximal 0,02 MPa. Geringfügig größere Unterschiede sind auf $\mathrm{SH}_{\text {kons }}(-0,03 \mathrm{MPa})$ und $\mathrm{SH}_{\text {konv }}(+0,04 \mathrm{MPa})$ festzustellen, aber auch sie bleiben deutlich unterhalb der Signifikanzgrenze (vgl. Tabelle A 43).

Bei Varianten mit $7,5 \mathrm{Mg}$ Radlast führt eine Erhöhung des RID zu einem Anstieg der hEW um durchschnittlich +0,02 MPa (vgl. Tabelle A 44). Das Jahr $2006(+0,05 \mathrm{MPa})$, die Fläche $\mathrm{SH}_{\text {kons }}(+0,06 \mathrm{MPa})$ und der Messtiefenbereich $18-27 \mathrm{~cm} \quad(+0,05 \mathrm{MPa})$ weisen zwar erkennbare Unterschiede auf, diese bleiben jedoch nicht statistisch signifikant. Auf den schleswig-holsteinischen Versuchsflächen ergeben sich bei konservierender Bodenbearbeitung bis 
$27 \mathrm{~cm}$ Messtiefe keine Unterschiede bei verändertem RID. Eine Erhöhung in $30-39 \mathrm{~cm}$ führt hingegen zu einer Abnahme des hEW um -0,07 MPa. Auf $\mathrm{SH}_{\text {konv }}$ steigt mit zunehmender Messtiefe der Einfluss des RID: in $5-14 \mathrm{~cm}$ nimmt der hEW mit höherem RID um +0,03 MPa zu, in $18-27 \mathrm{~cm}$ beträgt die Differenz +0,06 MPa, in $30-39 \mathrm{~cm}$ steigt sie auf $+0,09 \mathrm{MPa}$ (vgl. Abbildung 30).

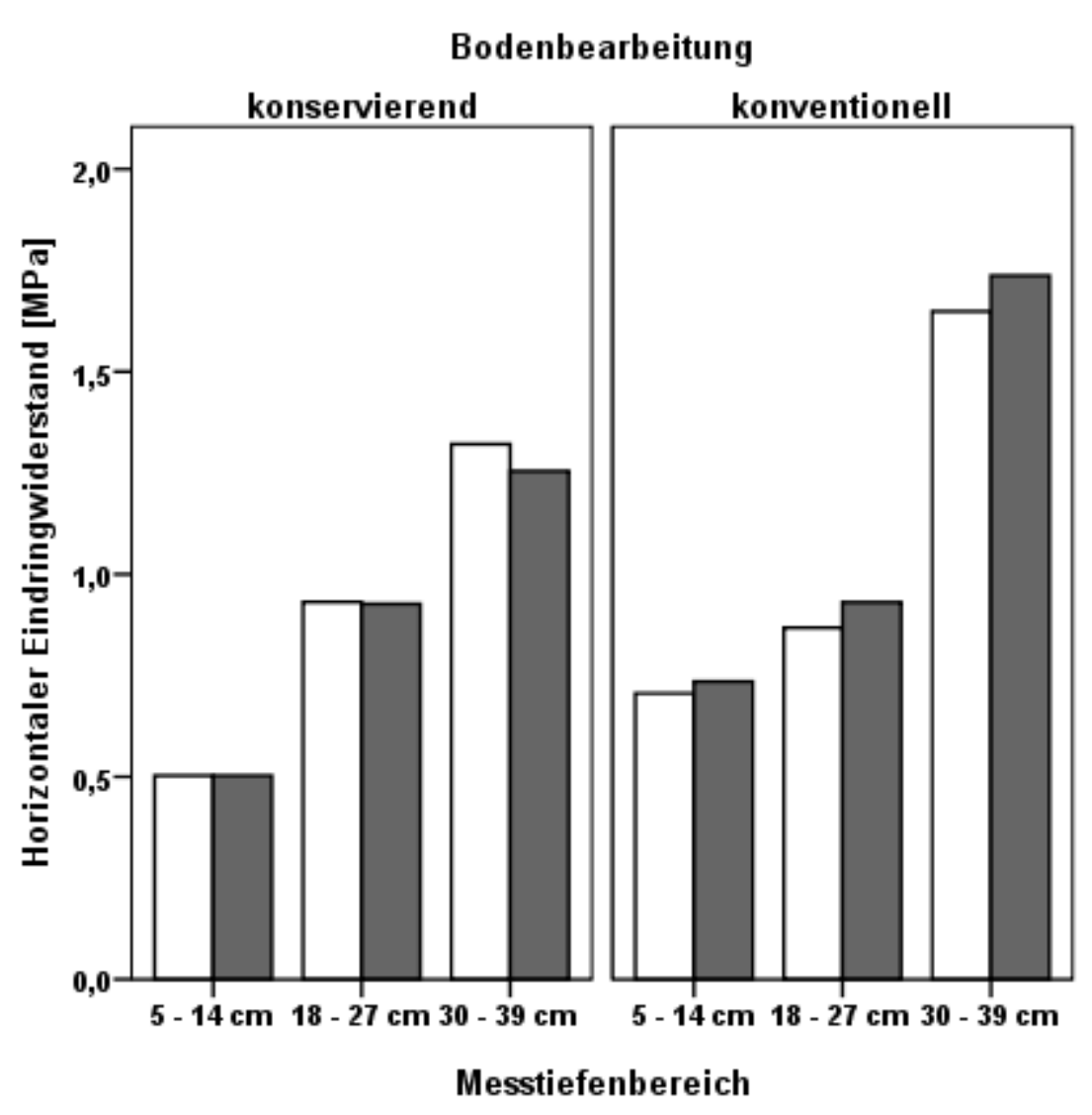
Variante
$\square 7,5 \mathrm{Mg} @ 250 \mathrm{kPa}$
$\square 7,5 \mathrm{Mg} @ 35 \mathrm{kPa}$

Abbildung 30: Effekte einer Erhöhung des Reifeninnendruckes bei 7,5 Mg Radlast auf den horizontalen Eindringwiderstand [MPa] bei konservierender und konventioneller Bodenbewirtschaftung am Standort Schleswig-Holstein $\left(n=510, \mathrm{GD}_{5 \%} \approx 0,12 \mathrm{MPa}, \overline{\mathrm{x}}\right.$ über 3 Termine, 2 Blöcke, 6 Wdh.).

\subsubsection{Der Sonderbereich Geophysik}

Erst im Jahr 2008 und nach Abschluss aller Untersuchungen des geophysikalischen Institutes konnte der Sonderbereich GEO mit TASIS beprobt 
werden. Aufgrund des geringen Stichprobenumfangs wurde allerdings darauf verzichtet, die Daten statistisch auszuwerten.

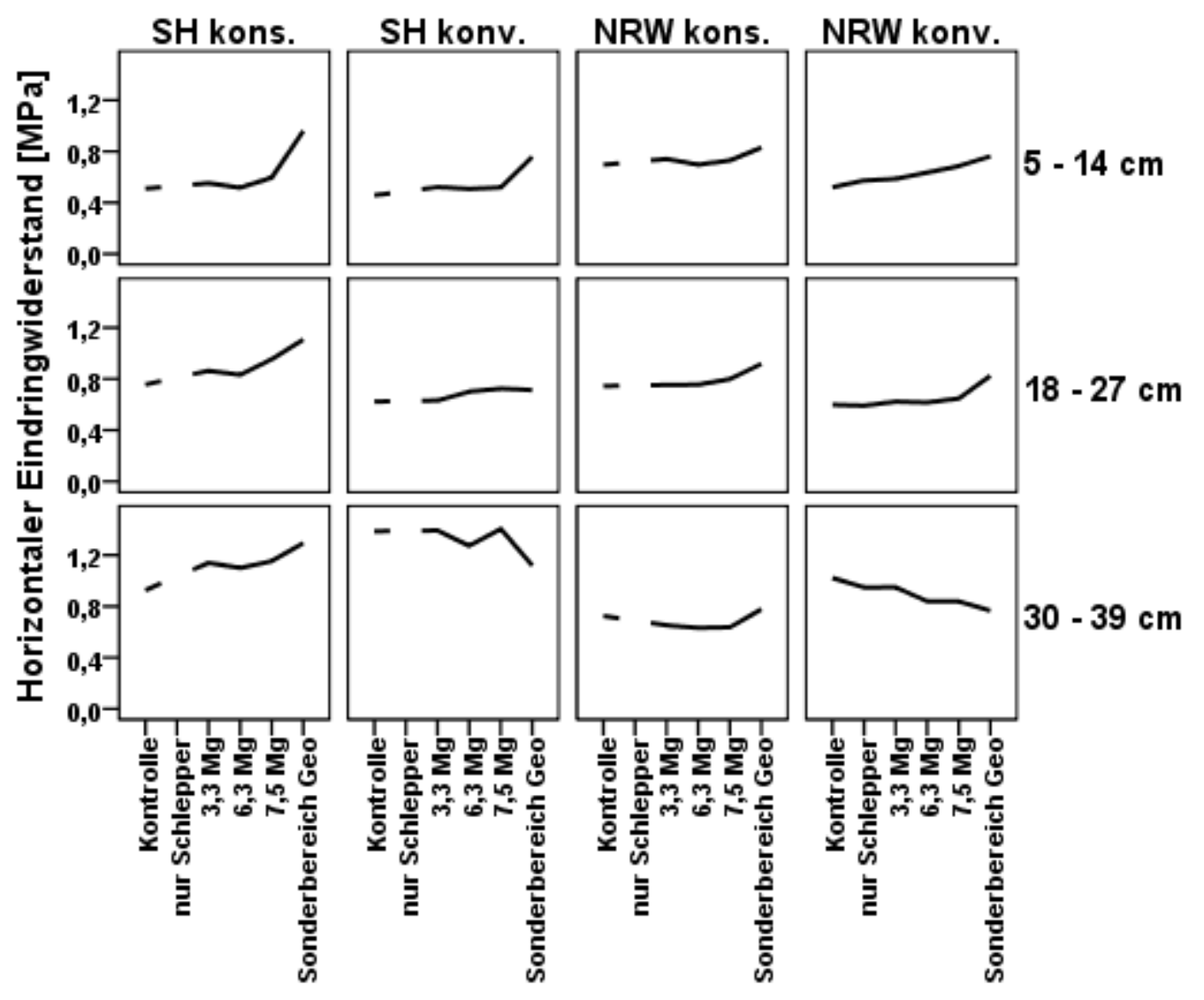

Abbildung 31: Trenddiagramm der horizontalen Eindringwiderstände [MPa] nach Versuchsfläche, Messtiefenbereich und Radlast unter Berücksichtigung des Sonderbereiches GEO (nur Daten aus 2008).

Die Datentrends sind in Abbildung 31 zusammengefasst und zeigen die Sonderstellung, die die Messwerte des Sonderbereiches Geophysik einnehmen. In 11 der 12 dargestellten Fälle weist GEO die Extrema der Messwerte auf, in 9 Fällen wurde für die Variante GEO der jeweils höchste hEW ermittelt. Die Bedingungen, unter denen für GEO die niedrigsten hEW gemessen werden konnten, beschränken sich auf Flächen unter konventioneller Bewirtschaftung und bei diesen auf den Messtiefenbereich $30-39 \mathrm{~cm}$. In den oberen Messtiefenbereichen nehmen die Werte mit steigender Radlast zu. 
In den Messtiefenbereichen bis $27 \mathrm{~cm}$ nehmen die hEW mit steigender Belastung unabhängig vom Bodenbearbeitungsverfahren zu. Unterschiede unter den Bodenbewirtschaftungsverfahren treten erst in $30-39 \mathrm{~cm}$ auf $(\mathrm{vgl}$. Abbildung 32).

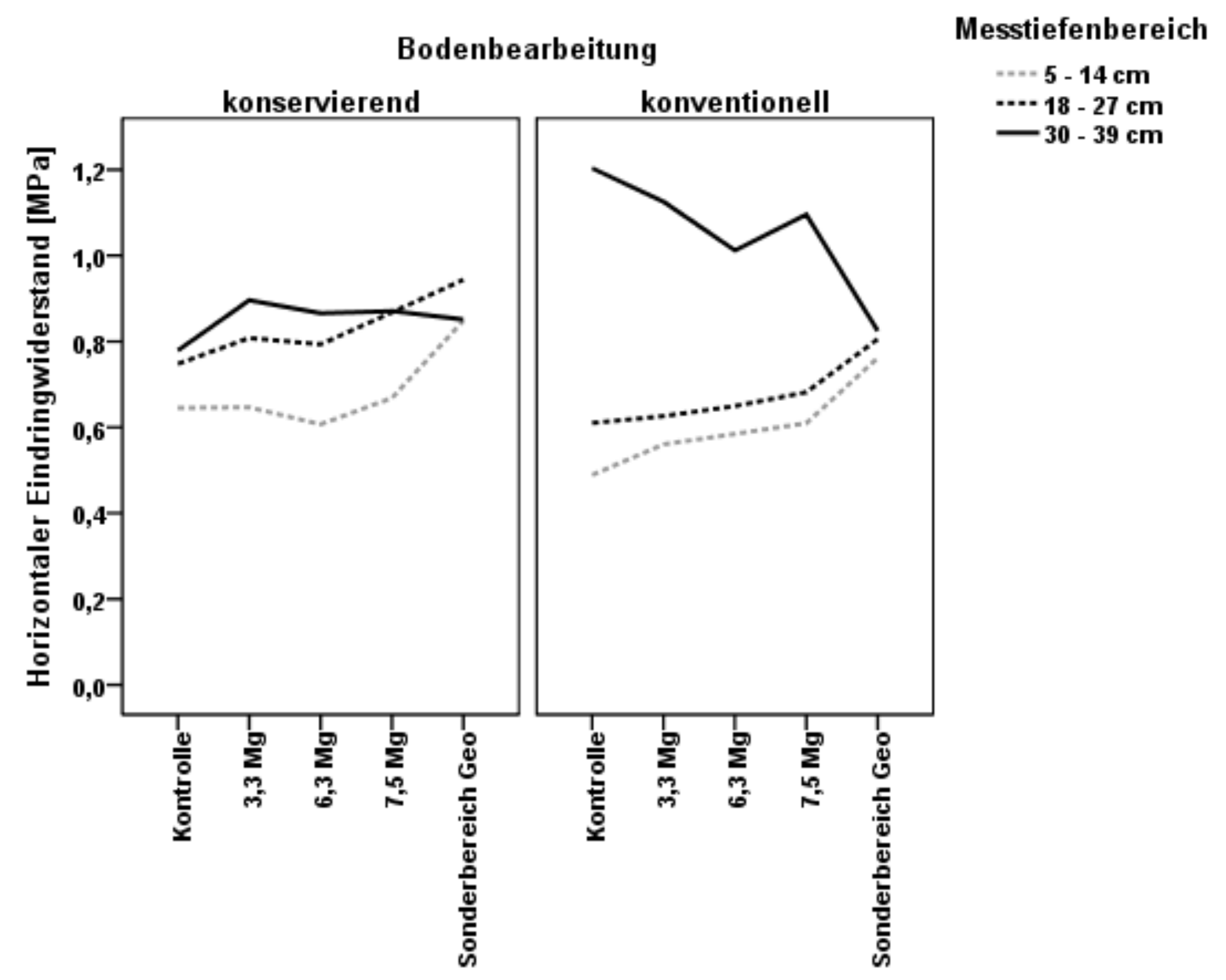

Abbildung 32: Horizontale Eindringwiderstände [MPa] nach Messtiefenbereich und Radlast für konservierende und konventionelle Bodenbearbeitung (Frühjahr 2008, ohne nur Schlepper).

Bei konservierender Bodenbearbeitung treten die Unterschiede von GEO $(+31,5 \%)$ gegenüber der Kontrolle besonders deutlich in $5-14 \mathrm{~cm}$ hervor, da die Varianten innerhalb des geordneten Versuchsdesigns maximale Unterschiede von $-5,9 \%$ (6,3 Mg), bzw. $+3,7 \%$ (7,5 Mg) aufweisen (vgl. 
Tabelle A 39). In $18-27 \mathrm{~cm}$ ist der Unterschied von GEO gegenüber der Kontrolle noch immer deutlich, jedoch fällt die Differenz mit $+26,2 \%$ geringer aus, und auch der Abstand gegenüber den Parzellen mit 7,5 Mg Radlast, die um $+16,1 \%$ höhere hEW aufweisen, ist weniger stark ausgeprägt als in 5 $14 \mathrm{~cm}$. In $30-39 \mathrm{~cm}$ Tiefe können bei konservierender Bodenbewirtschaftung zwar generell höhere hEW in befahrenen Bereichen festgestellt werden, ein Unterschied von GEO gegenüber anderen Radlastvarianten ist jedoch nicht erkennbar: die Differenzen betragen hier maximal 0,05 MPa.

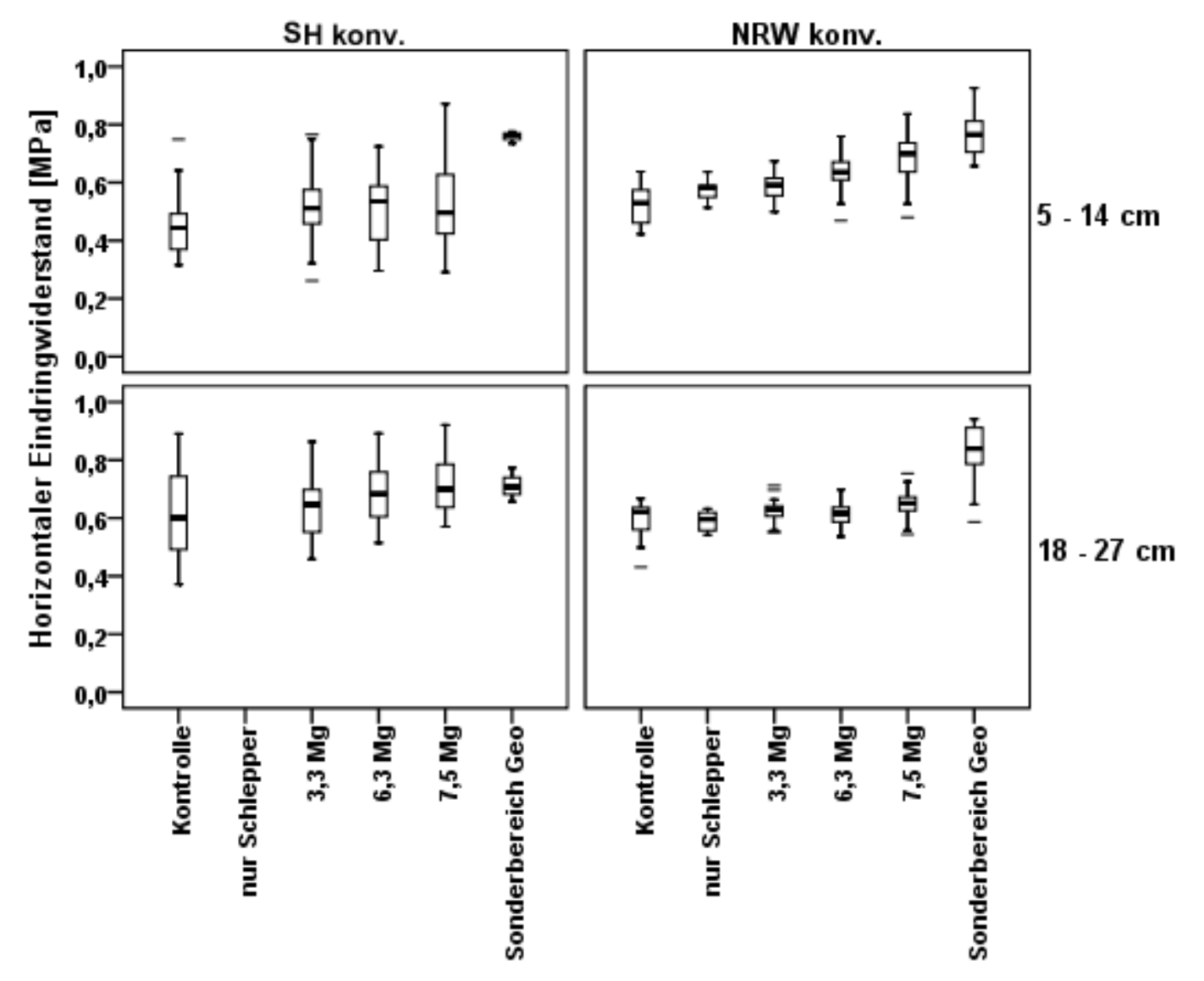

Abbildung 33: Entwicklung der horizontalen Eindringwiderstände [MPa] auf konventionell bewirtschafteten Flächen bei steigender mechanischer Belastung in den Messtiefenbereichen $5-14 \mathrm{~cm}$ und $18-27 \mathrm{~cm}$.

Auf Flächen mit konventioneller Bodenbearbeitung zeigt sich eine andere Entwicklung der Messwerte, die besonders deutlich auf $\mathrm{NRW}_{\text {konv }} \mathrm{zu}$ beobachten 
ist (vgl. Abbildung 33 und Abbildung 34): Während im Bereich $5-14 \mathrm{~cm}$ eine stetige Zunahme der hEW mit steigenden Radlasten von 0,52 MPa (Kontrolle) bis $0,76 \mathrm{MPa}$ (GEO) zu beobachten sind, kann im Bereich $30-39 \mathrm{~cm}$ eine kontinuierliche Abnahme der $\mathrm{hEW}$ von der Kontrolle (1,02 MPa) über die Variante $3,3 \mathrm{Mg}(0,95 \mathrm{MPa}), 6,3 \mathrm{Mg}$ und $7,5 \mathrm{Mg}$ (jeweils $0,84 \mathrm{Mg}$ ) bis GEO $(0,77 \mathrm{MPa})$ festgestellt werden.

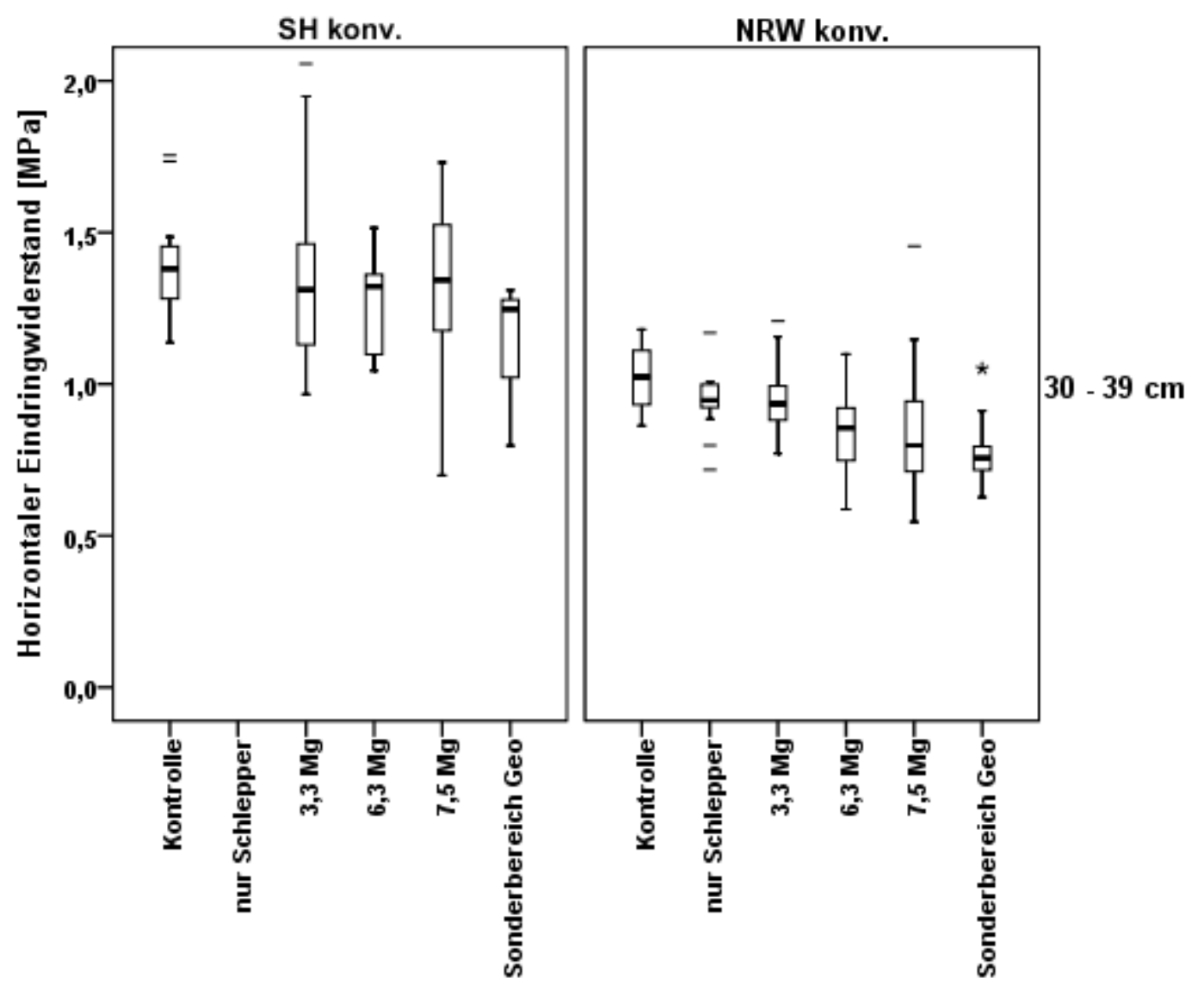

Abbildung 34: Entwicklung der horizontalen Eindringwiderstände [MPa] auf konventionell bewirtschafteten Flächen bei steigender mechanischer Belastung in den Messtiefenbereichen $30-39 \mathrm{~cm}$.

Auf $\mathrm{SH}_{\text {konv }}$ konnte diese Beobachtung gleichermaßen gemacht werden: während die hEW von GEO in $5-14 \mathrm{~cm}$ gegenüber der Kontrolle um $+65,8 \%$ und in $18-27 \mathrm{~cm}$ um $+16,1 \%$ erhöht waren, wiesen die Daten in $30-39 \mathrm{~cm}$ bei 
insgesamt uneinheitlichem Trend mit $-19,4 \%$ die niedrigsten Messwerte gegenüber der Kontrolle aus.

\subsubsection{Untersuchungen zur Eingriffsintensität von TASIS in das Bodengefüge}

Für die bisherige Datenaufbereitung wurden nur diejenigen TASIS-Messdaten berücksichtigt, die bei den Erstmessungen eines jeden Jahres erhoben wurden. Um eine Bewertung der Eingriffsintensität von TASIS in das Bodengefüge vornehmen zu können, werden in einem zweiten Analyseschritt alle Messdaten berücksichtigt, die erhoben wurden, d. h. auch diejenigen, die in 2007 bei einer zweiten Messung bzw. 2008 bei einer zweiten oder dritten Messung erhoben wurden. Daten aus 2006 wurden nicht berücksichtigt.

Der durchschnittliche hEW bei einem Ersteinsatz von TASIS betrug 0,84 MPa und lag damit 6,5\% über jenen TASIS-Messwerten, die bei der zweiten Durchdringung des Bodens im darauf folgenden Jahr (0,79 MPa) bzw. 26,1\% über jenen, die bei der dritten Messfahrt an selber Stelle in 2008 (0,67 MPa) ermittelt wurden. Je nach Fläche und Jahr unterschieden sich die gewonnenen Messwerte (vgl. Tabelle A 45): in 2007 waren die hEW bei einem Ersteinsatz von TASIS gegenüber den aus dem Zweiteinsatz gewonnen Daten auf den

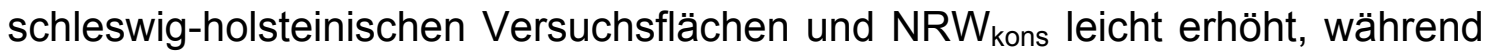
sie auf $\mathrm{NRW}_{\text {konv }}$ leicht unter jenen des zweiten TASIS-Einsatzes lagen.

Die Messdaten aus 2008 erlauben den Vergleich von Erst-, Zweit- und Drittmessungen und ergeben bereits ein differenzierteres Bild (vgl. Abbildung 35 und Tabelle $\mathrm{A}$ 46). Auf $\mathrm{SH}_{\text {konv }}, \mathrm{SH}_{\text {kons }}$ und $\mathrm{NRW}_{\text {kons }}$ wurden die höchsten hEW jeweils bei den Erstmessungen ermittelt. Sie lagen durchschnittlich bei 0,81 MPa und somit +0,11 MPa über jenen der zweiten bzw. +0,14 MPa über jenen der dritten Durchfahrt. Weiter ist erkennbar, dass sich die Unterschiede nach Bodenbearbeitung und Messtiefenbereich differenzieren lassen: Für beide Bodenbewirtschaftungssysteme zeigen sich nur sehr geringe Änderungen des hEW (max. -0,02 MPa) im Bereich $5-14 \mathrm{~cm}$. Dieses ist auf den Flächen unter Pflug auch noch in $18-27 \mathrm{~cm}$ der Fall, während bei pflugloser Bodenbearbeitung bereits ein signifikanter Unterschied von 0,10 MPa zwischen den 
Werten des ersten und des zweiten TASIS-Einsatzes zu erkennen ist. Ein weiteres Absinken des hEW zwischen zweiter und dritter Messfahrt ist jedoch nicht zu beobachten. Dieselbe Ausprägung dieses Merkmals findet sich auch in 30 - 39 cm Messtiefe. Hier beträgt der Unterschied des hEW des zweiten TAsIsEinsatzes gegenüber jenem der Erstmessung -0,23 MPa oder -26,7\%. Die Drittmessung hingegen ist der zweiten Messung nahezu identisch.

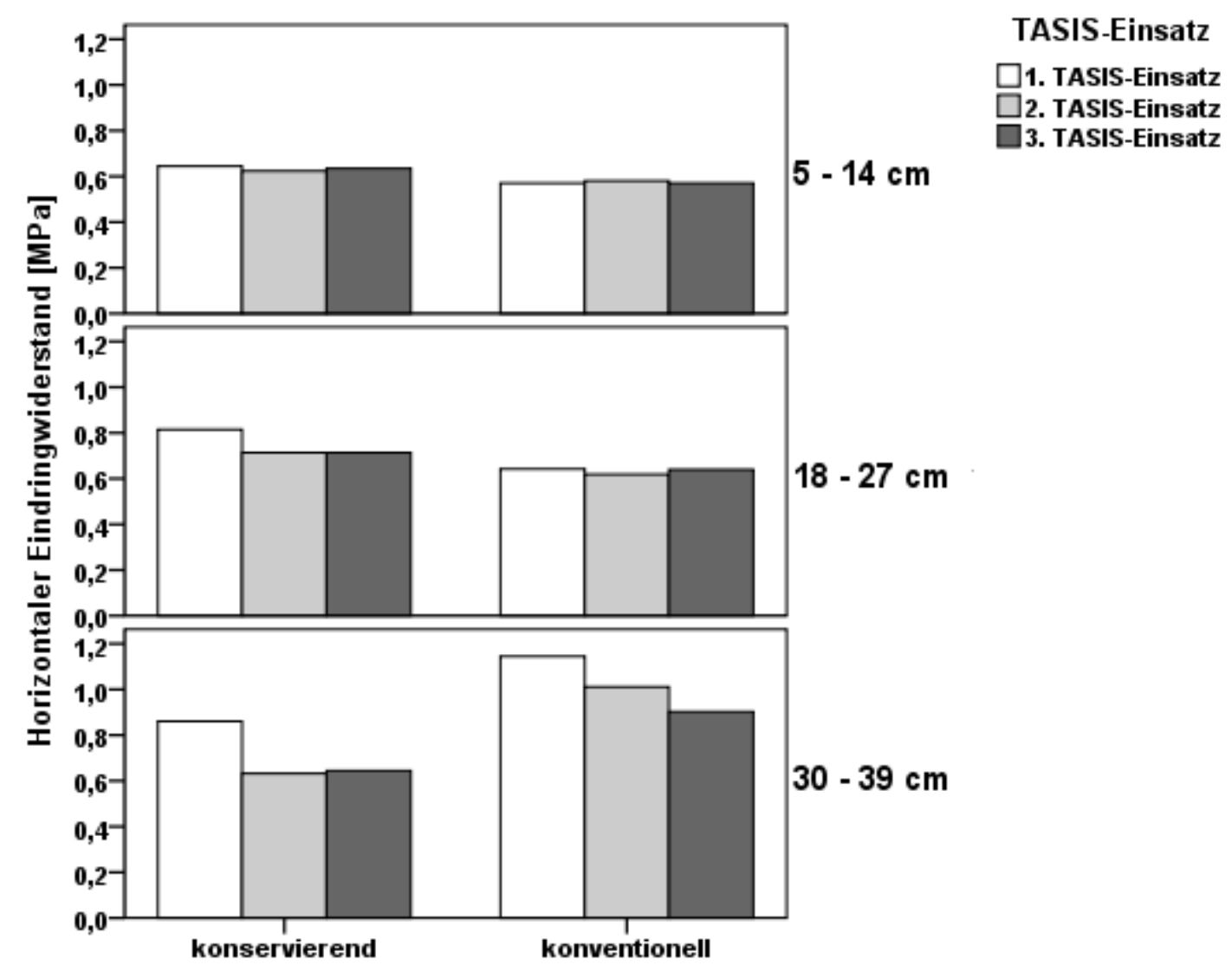

Abbildung 35: Horizontale Eindringwiderstände [MPa] in 2008 nach Bodenbewirtschaftung und Messtiefenbereich in Abhängigkeit des TASIS-Einsatzes an selber Stelle $\left(n=3.948, G_{5 \%} \approx 0,03 \mathrm{MPa}, \bar{x}\right.$ über 2 Standorte, 6 Varianten, 2 Blöcke, 6 Wdh.).

Bei konventioneller Bodenbearbeitung kann in $30-39 \mathrm{~cm}$ Messtiefe auch noch beim dritten TASIS-Einsatz (0,90 MPa) eine Abnahme der hEW gegenüber der zweiten Messung (1,01 MPa) festgestellt werden. Die höchsten hEW $(1,14 \mathrm{MPa})$ wurden bei der Erstmessung ermittelt. Die Flächen $\mathrm{SH}_{\text {konv }}$ und 
$N R W_{\text {konv }}$ unterscheiden sich jedoch erheblich in Bezug auf ihre Ergebnisse: auf $\mathrm{NRW}_{\text {konv }}$ wurden für alle drei Messfahrten nahezu identische Messwerte ermittelt $(0,71 \mathrm{MPa}-0,71 \mathrm{MPa}-0,72 \mathrm{MPa})$, wohingegen am Standort Schleswig-Holstein die Messwerte deutlich differenzierter ausfielen (0,96 MPa $0,77 \mathrm{MPa}-0,56 \mathrm{MPa})$.

\subsection{Betrachtung der Zusammenhänge von vertikalen und horizontalen Eindringwiderständen}

Nachfolgend soll untersucht werden, ob zwischen vertikalen und horizontalen Eindringwiderständen ein Zusammenhang hergestellt werden kann. Hierzu werden zunächst Korrelationen nach Pearson errechnet. Anschließend wird der Ruhedruckkoeffizient als rechnerische Größe aus beiden Messwerten abgeleitet, und seine Eignung zur Bewertung der Sachverhalte wird analysiert.

\subsubsection{Betrachtung der Korrelationen zwischen vertikalen und horizontalen Eindringwiderständen}

Die Korrelation des Gesamtdatensatzes ohne GEO beträgt $r=0,60$. Das Jahr 2007 weist hierbei einen leicht schwächeren Korrelationskoeffizienten aus $(r=0,56)$ als $2008(r=0,61)$. Die Werte korrelieren auf konservierend bewirtschafteten Flächen leicht schwächer $(r=0,541)$ als auf konventionell bewirtschafteten $(r=0,654)$. NRW kons bei der Analyse des Koeffizienten nach der Fläche die erkennbar schwächste Korrelation $(r=0,36)$ auf ( $v g l$. Abbildung 36). Alle genannten Korrelationskoeffizienten sind signifikant. 


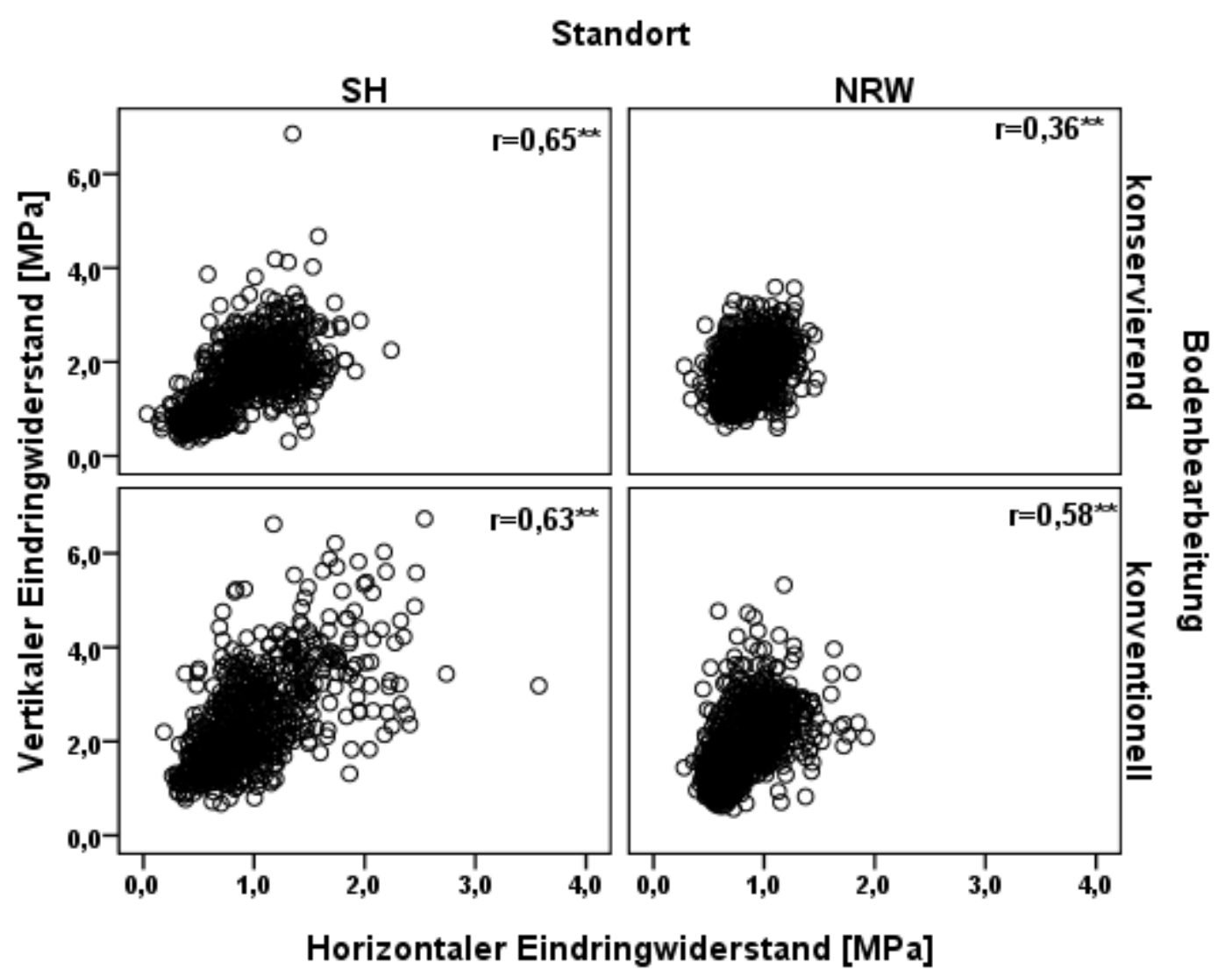

Abbildung 36: Punktwolken-Diagramme mit Pearson-Korrelationskoeffizienten $\left(^{* *} \triangleq\right.$ signifikant mit $\left.p \leq 0,01\right)$ nach Standort und Bodenbearbeitungssystem $\left(r_{\text {gesamt }}=0,60^{\star *}, n=3.773, \bar{x}\right.$ über 3 Termine, 3 Messtiefen, 6 Varianten, 2 Blöcke, 6 Wdh.)

In einer detailierteren Betrachtung, die auch die Messtiefenbereiche nach Fläche berücksichtigt, treten z. T. deutlich schwächere Korrelationen auf (vgl. Tabelle 7). 
Tabelle 7: Pearson-Korrelationskoeffizienten $(* * \wedge$ signifikant mit $p \leq 0,01)$ zwischen horizontalem und vertikalem Eindringwiderstand nach Versuchsfläche und Messtiefenbereich ( $\bar{x}$ über 3 Termine, 6 Varianten, 2 Blöcke, $6 \mathrm{Wdh}$.)

\begin{tabular}{lllll}
\hline MTB [cm] & $\mathrm{SH}_{\text {kons }}$ & $\mathrm{SH}_{\text {konv }}$ & $\mathrm{NRW}_{\text {kons }}$ & $\mathrm{NRW}_{\text {konv }}$ \\
\hline $5-14$ & $0,230^{* *}$ & $0,397^{* *}$ & $0,062^{\text {n.s. }}$ & $-0,042^{\text {..s. }}$ \\
& $\mathrm{n}=274$ & $\mathrm{n}=272$ & $\mathrm{n}=300$ & $\mathrm{n}=432$ \\
$18-27$ & $0,185^{* *}$ & $0,284^{* *}$ & $0,282^{* *}$ & $0,040^{\text {n.s. }}$ \\
& $\mathrm{n}=274$ & $\mathrm{n}=271$ & $\mathrm{n}=300$ & $\mathrm{n}=432$ \\
$30-39$ & $0,054^{\text {n.s. }}$ & $0,233^{* *}$ & $0,146^{*}$ & $0,082^{\text {n.s. }}$ \\
& $\mathrm{n}=274$ & $\mathrm{n}=176$ & $\mathrm{n}=300$ & $\mathrm{n}=432$ \\
\hline n.s.: & nicht signifikant & & \\
$*:$ & signifikant mit $\mathrm{p} \leq 0,05$ & & & \\
$* *:$ & signifikant mit $\mathrm{p} \leq 0,01$ & & &
\end{tabular}

Auf $\mathrm{NRW}_{\text {konv }}$ korrelieren die Werte nahezu überhaupt nicht miteinander und weisen in keiner Messtiefe einen Korrelationskoeffizienten größer 0,08 aus. Auf $\mathrm{SH}_{\text {konv }}$ zeigen sich über die Messtiefen mäßige Korrelationen mit Koeffizienten zwischen 0,23 $(30-30 \mathrm{~cm})$ und 0,40 $(5-14 \mathrm{~cm})$, die über die Tiefe abnehmen. Auch auf der konservierend bewirtschafteten Fläche am Standort SH geht die Korrelation der beiden Messgrößen über die Tiefe zurück, allerdings auf einem erkennbar niedrigeren Niveau mit Koeffizienten zwischen 0,23 $(5-14 \mathrm{~cm})$ und $0,05(30-39 \mathrm{~cm})$. Auch auf $\mathrm{NRW}_{\text {kons }}$ kann insgesamt nur ein schwacher Zusammenhang in den einzelnen Messtiefen ermittelt werden. In $18-27 \mathrm{~cm}$ erreicht der Korrelationskoeffizient sein Maximum, und im Bereich des Oberbodens lässt sich nahezu keine Korrelation feststellen.

\subsubsection{Der Ruhedruckkoeffizient als rechnerische Größe aus horizontalem und vertikalem Eindringwiderstand}

Wird die im Boden vorherrschende horizontale Hauptspannung $\sigma_{1}$ auf die vertikale Hauptspannung $\sigma_{2}$ bezogen, so ergibt sich hieraus als rechnerische Größe der dimensionslose Ruhedruckkoeffizient (RDK) oder Ruhedruckbeiwert (HARTGE \& HORN 1999). Im Rahmen der durchgeführten Untersuchungen bieten die Vertikal- und Horizontalpenetrometermessungen die Datenbasis, um eine solche Bezugsstellung durchzuführen. Den sechs VP-Einzelmessungen in einer 
jeden Parzelle werden die sechs Einzeldaten der TASIS-Ersteinsätze des jeweiligen Jahres gegenübergestellt, um so sechs RDK für eine Parzelle zu gewinnen. Aufgrund des sehr eingeschränkten Stichprobenumfangs für die Sonderflächen GEO erfolgt deren Betrachtung getrennt vom eigentlichen Hauptdatensatz und ohne statistische Analyse.

Nach FAZEKAS ET AL. (2005) weist ein normal verdichteter Boden einen RDK zwischen 0,2 und 0,7 aus. Ein Boden kann als überverdichtet angesehen werden, wenn sich der RDK dem Wert von 1 nähert, da dann die horizontale Hauptspannung größer ist als die vertikale.

Ruhedruckkoeffizient [-], Eindringwiderstand [MPa]

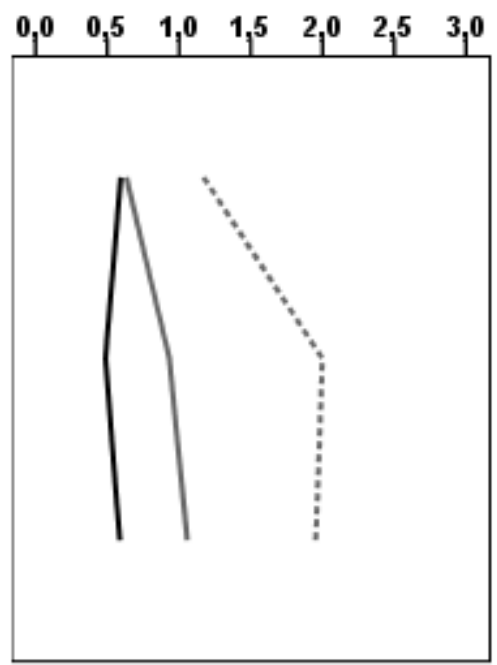

konservierend

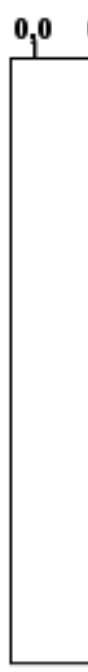

\section{Bodenbearbeitung}

Abbildung 37: Ruhedruckkoeffizienten und horizontale und vertikale Eindringwiderstände nach Bodenbearbeitung und Messtiefenbereich $\left(\mathrm{GD}_{5 \%} \approx 0,02, \overline{\mathrm{x}}\right.$ über 2 Standorte, 3 Termine, 6 Varianten, 2 Blöcke, 6 Wdh.).

Aus den im Rahmen des Versuchsdesigns gewonnenen Daten können RDK ermittelt werden, die sich im vorgegebenen Wertebereich eines normal verdichteten Bodens bewegen. Die Standorte Kiel und Soest weisen mit 0,52 bzw. 0,49 ein vergleichbares Messwertniveau aus, wobei auf den konservierend bewirtschafteten Flächen jeweils die höheren RDK errechnet werden. Ist der Unterschied zwischen $\mathrm{NRW}_{\text {kons }}(0,51)$ und $\mathrm{NRW}_{\text {konv }}(0,48)$ noch relativ gering, 
so fällt er auf den schleswig-holsteinischen Flächen mit 0,60 ( $\left.\mathrm{SH}_{\text {kons }}\right)$ und 0,44 $\left(\mathrm{SH}_{\text {konv }}\right)$ bereits stärker ins Gewicht.

Bei Flächen unter konservierender Bodenbearbeitung bestehen zwischen oberstem und unterstem Messtiefenbereich keine Unterschiede zwischen den ermittelten RDK (jeweils 0,59), wohingegen die Werte in $18-27 \mathrm{~cm}$ aufgrund der relativ stärker ansteigenden vEW in dieser Messtiefe auf 0,49 MPa abfallen. Auf konventionell bewirtschafteten Flächen verzeichnen sowohl horizontale als auch vertikale EW einen charakteristischen Anstieg der Messwerte in 30 $39 \mathrm{~cm}$ (vgl. Abbildung 37). Da sich dieser jedoch für beide Messgrößen in nahezu identischer Größenordnung ausprägt, bleibt der RDK als rechnerische Größe hiervon unberührt und auf nahezu konstantem Niveau. 


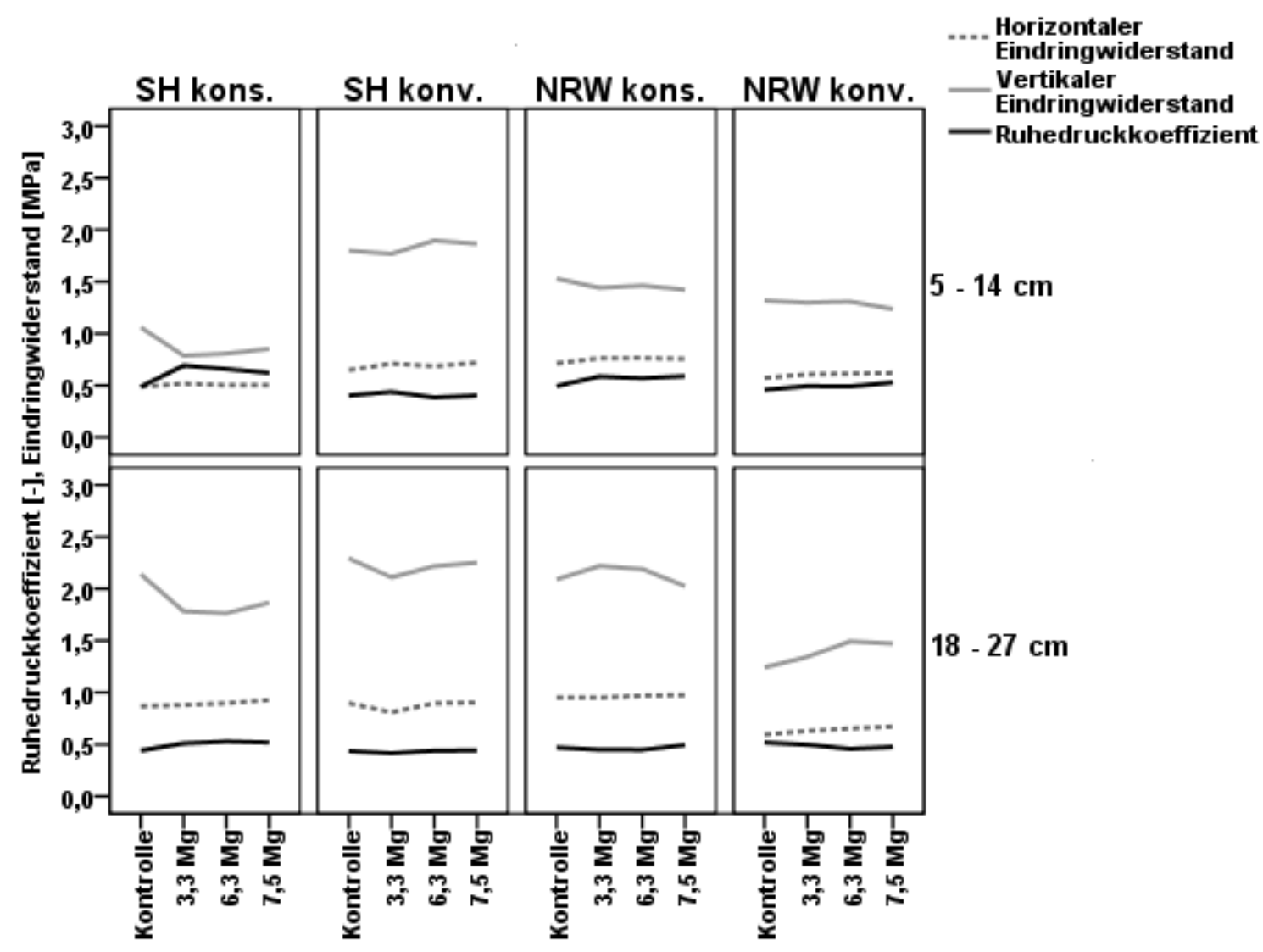

Abbildung 38: Ruhedruckkoeffizienten und horizontale und vertikale Eindringwiderstände nach Versuchsfläche und Radlast in den Messtiefen $5-14 \mathrm{~cm}$ und 18 - $27 \mathrm{~cm}\left(\mathrm{GD}_{5 \%} \approx 0,05, \bar{x}\right.$ über 3 Termine, 2 Reifeninnendrücke je Radlast, $12 \mathrm{Wdh}$.).

Abbildung 38 zeigt in der weitergehenden Betrachtung der Daten, wie sich die RDK und die horizontalen bzw. vertikalen EW in Abhängigkeit von Fläche und Messtiefe bei verschiedenen Radlasten verhalten. Die Werte bewegen sich zwischen einem Minimum von 0,38 und einem Maximum von 0,73 (vgl. auch Tabelle A 47).

Im Messbereich 5-14 cm der konservierend bewirtschafteten Flächen und

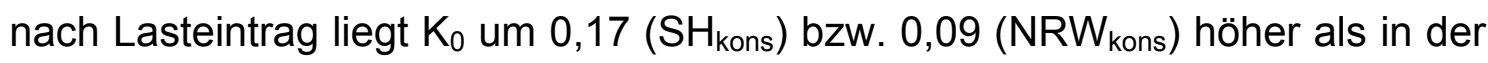
Kontrolle. In tiefer liegenden Bereichen zeigen die Daten eine abnehmende Dynamik. Auf den konventionell bewirtschafteten Flächen hingegen kann eine umgekehrte Entwicklung beobachtet werden: hier nimmt $\mathrm{K}_{0}$ um 0,04 ( $\left.\mathrm{SH}_{\mathrm{konv}}\right)$

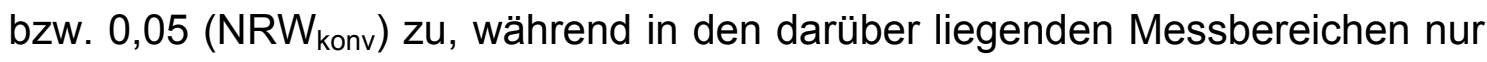
marginale Änderungen auftreten. 
Wie oben dargestellt, resultieren erhöhte EW nicht zwangsweise in erhöhten RDK: in $30-39 \mathrm{~cm}$ Messtiefe weist $\mathrm{SH}_{\text {konv }}$ mit durchschnittlichen vEW von 3,51 MPa die mit Abstand höchsten Werte aus, da dieses jedoch auch für den hEW (1,64 MPa) der Fall ist, nimmt der RDK mit 0,49 einen moderaten Wert ein. Aufgrund der weniger stark schwankenden Werte, die mit TASIS ermittelt wurden, sind es zumeist die vEW, deren Werte jene des RDK maßgeblich beeinflussen, was in der Messtiefe $30-39 \mathrm{~cm}$ deutlich anhand des Verlaufes verschiedener Messwertlinien erkennbar ist.

\subsection{Bodenkundliche Untersuchungen}

Um die mit dem Vertikalpenetrometer und TASIS erhobenen Daten besser bewerten zu können, wurden nach Ablauf des Versuches auf der Fläche $N R W_{\text {konv }}$ ergänzende bodenkundliche Untersuchungen in den Bodentiefen 36 $40 \mathrm{~cm}$ und $60-64 \mathrm{~cm}$ durchgeführt. Beprobt wurden die Kontrollfläche, die innerhalb des Versuchsdesigns gelegene Variante 7,5Mg@350kPa und die Sonderfläche GEO. Die Beprobungen beinhalteten die Entnahme von Stechzylinderproben und Spatendiagnosen, die von Thomas Weyer aus dem Fachbereich Agrarwirtschaft der FH-SWF begleitet wurden. Die Ergebnisse der Stechzylinderuntersuchungen befinden sich in Tabelle 8, jene der Spatendiagnose in Tabelle 9 bzw. Tabelle A 49. 
Tabelle 8: Ergebnisse der Stechzylinderproben der Versuchfläche NRW $_{\text {konv }}$ (Mittelwerte, $\mathrm{n}=\mathbf{2 0}$ )

\begin{tabular}{llccc}
\hline Tiefe [cm] & Daten & Kontrolle & $7,5 \mathrm{Mg} @ 350 \mathrm{kPa}$ & $\begin{array}{c}\text { Sonderbereich } \\
\text { GEO }\end{array}$ \\
\hline \multirow{3}{*}{ - 40 } & Lagerungsdichte [g/cm ${ }^{3}$ ] & 1,54 & 1,57 & 1,58 \\
& Gesamtporenvolumen [Vol.-\%] & 40,05 & 38,51 & 38,38 \\
& weite Grobporen [Vol.-\%] & 4,58 & 2,17 & 2,42 \\
& enge Grobporen [Vol.-\%] & 3,66 & 4,27 & 3,10 \\
& mittlere Poren [Vol.-\%] & 20,53 & 20,60 & 21,32 \\
& Feinporen [Vol.-\%] & 11,28 & 11,48 & 11,54 \\
& Porenziffer & 0,67 & 0,63 & 0,62 \\
& kf-Wert & 44,70 & 27,10 & 14,40 \\
& Lagerungsdichte [g/cm ${ }^{3}$ ] 64 & 1,53 & 1,50 & 1,51 \\
& Gesamtporenvolumen [Vol.-\%] & 40,40 & 41,62 & 41,66 \\
& weite Grobporen [Vol.-\%] & 4,99 & 5,63 & 5,33 \\
& enge Grobporen [Vol.-\%] & 3,76 & 4,61 & 4,51 \\
& mittlere Poren [Vol.-\%] & 18,19 & 19,06 & 18,90 \\
& Feinporen [Vol.-\%] & 13,46 & 12,32 & 12,92 \\
& Porenziffer & 0,68 & 0,72 & 0,71 \\
& kf-Wert & 103,40 & 153,40 & 30,40 \\
\hline
\end{tabular}

Im Bereich der oberen Beprobungstiefe $(36-40 \mathrm{~cm})$ sind bei einigen Parametern Unterschiede zwischen befahrenen und unbefahrenen Parzellen festzustellen. Die Datensätze des Sonderbereichs GEO sowie der Variante $7,5 \mathrm{Mg} @ 350 \mathrm{kPa}$ weisen geringfügig höhere Lagerungsdichten und um durchschnittlich -1,6 Prozentpunkte niedrigere Gesamtporenvolumina gegenüber der Kontrolle auf. Die Lagerungsdichten in den befahrenen Bereichen, obwohl geringfügig erhöht, liegen stets in einem Bereich, der nach Bodenkundlicher Kartieranleitung (AG BODEN 2005) als mittel $\left(1,45-1,65 \mathrm{~g} / \mathrm{cm}^{3}\right)$ einzustufen ist. Bei Betrachtung der Porenvolumina fällt jedoch die Abnahme der weiten Grobporen der befahrenen Parzellen gegenüber der Kontrolle auf. Nach LEBERT ET AL. (2004) sind Werte < $5 \%$ als kritisch zu betrachten und können auf Bodenverdichtungen hindeuten. Die Werte bewegen sich insgesamt auf einem niedrigen Niveau, und bereits der Mittelwert der Kontrolle (4,58 Vol.$\%)$ unterschreitet diesen Wert. Die Werte der befahrenen Parzellen liegen mit 2,17\% (7,5Mg@350kPa) und 2,42\% (Sonderbereich GEO) deutlich unterhalb des Schadschwellenwertes. In diesen Varianten können aber leicht erhöhte 
Werte für den Anteil der engen Grobporen bzw. der mittleren Poren gegenüber der Kontrolle festgestellt werden. Weiterhin auffällig ist in den befahrenen Bereichen eine geringere hydraulischen Leitfähigkeit: der kf-Wert der befahrenen Parzellen ist gegenüber der Kontrolle um 39,4\% (7,5Mg@350kPa) bzw. 67,8\% (Sonderbereich GEO) reduziert. Im Bereich des Unterbodens (60 $64 \mathrm{~cm}$ ) lassen die kf-Werte aufgrund vermehrter Regenwurmtätigkeit keine Aufschlüsse $\mathrm{zu}$, wie auch die weiteren Parameter nur sehr geringe Unterschiede zwischen den Varianten erkennen lassen. Lagerungsdichte und Gesamtporenvolumina unterscheiden sich kaum. Die befahrenen Parzellen weisen jedoch den höheren Anteil an weiten und engen Grobporen, die Kontrolle hingegen den höheren Feinporenanteil aus.

In Abbildung 39 findet sich eine Darstellung der Messwerte von Luftkapazitäten und gesättigten Wasserleitfähigkeiten als Punktwolken-Diagramm in Abhängigkeit von Variante und Beprobungstiefe. Die Schadschwellenwerte sind in Anlehnung an LEBERT ET AL. (2004) und CRAMER (2006) in Form gestrichelter Linien eingezeichnet: Oberhalb der fein gestrichelten Linie, die den kritischen Wert von $1 \mathrm{~cm}$ Wasserleitfähigkeit pro Tag markiert, werden Messwerte als unkritisch angesehen. Sie gelten dann als kritisch, wenn sie unterhalb dieses Wertes liegen und in Verbindung mit weiteren Indikatoren auftreten. Die kräftig gestrichelte senkrechte Linie markiert die Schadschwelle der Luftkapazität von 5 Vol.-\%. Links dieser Linie werden Messwerte nach dem Indikatorenmodell als kritisch angesehen, wenn sie in Verbindung mit weiteren Indikatoren auftreten. Die daraus resultierenden vier Quadranten werden von oben rechts beginnend entgegen dem Uhrzeigersinn gezählt, so dass Messwerte im ersten Quadranten keinen der beiden Schadschwellen unterschreiten, jene im dritten Quadranten hingegen beide. 


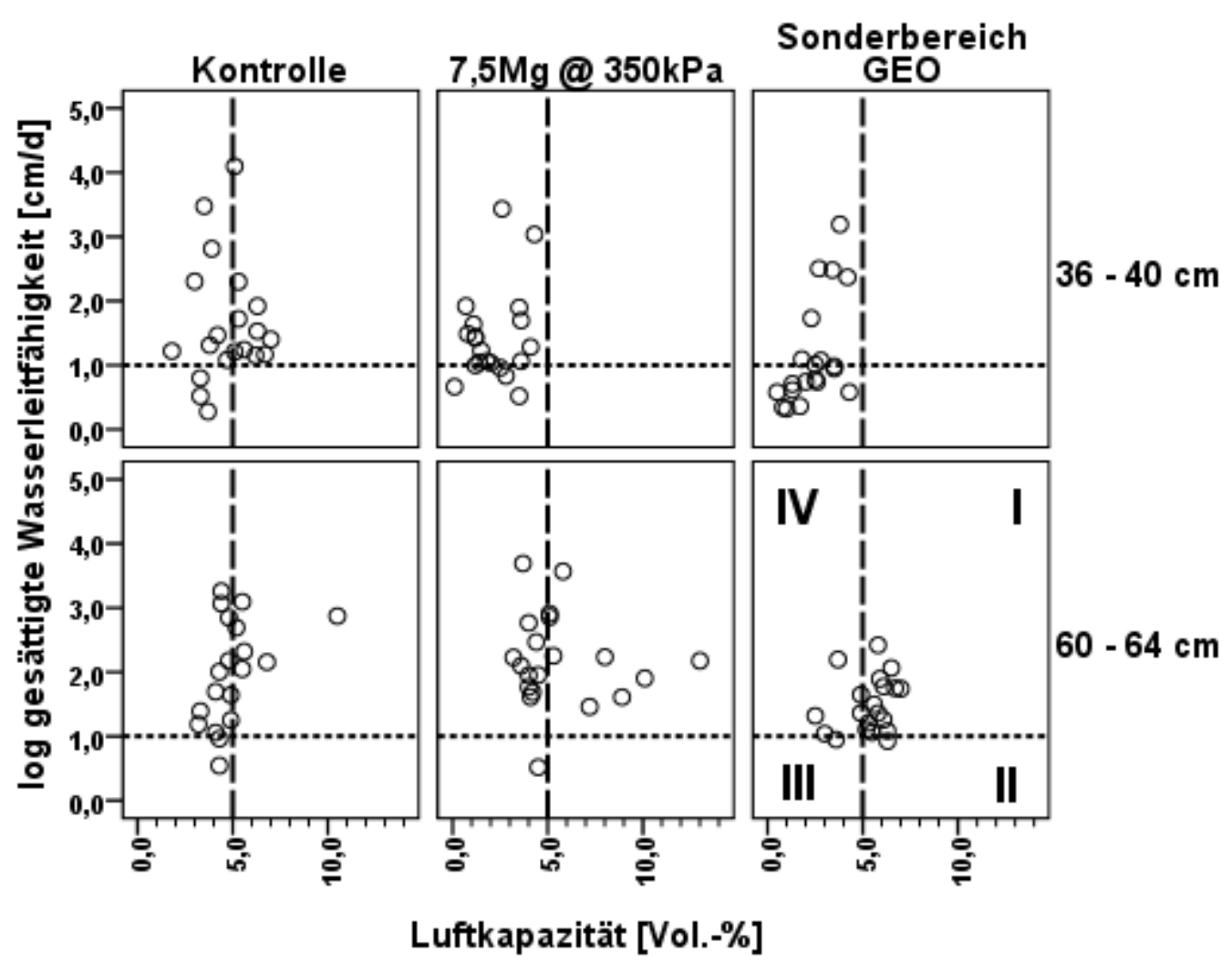

Abbildung 39: Luftkapazität (\%) und gesättigte Wasserleitfähigkeit $(\mathrm{cm} / \mathrm{d})$ von $\mathrm{NRW}_{\text {konv }}$ in Abhängigkeit von Belastungsvariante und Messtiefenbereich nach Versuchsende. Die gestrichelten Linien kennzeichnen die Schadschwellen nach LEBERT ET AL. 2004) $(n=20)$.

In Abbildung 39 zeigen sich im Bereich der Krumenbasis $(36-40 \mathrm{~cm})$ erkennbare Unterschiede der Kontrolle gegenüber den befahrenen Varianten sowohl bezogen auf die gesättigte Wasserleitfähigkeit als auch in Bezug auf die Luftkapazität: Die Mehrzahl der Messungen in der Kontrollparzelle liegt im Bereich des Schnittpunktes der eingezeichneten Grenzwert-Geraden. Nur bei drei Einzelmessungen werden beide Grenzwerte unterschritten: diese befinden sich im dritten Quadranten. Insgesamt acht Messwerte liegen im ersten Quadranten und überschreiten keinen der beiden Grenzwerte. Ein anderes Bild ergibt sich für die befahrenen Parzellen: hier liegen für beide Varianten sämtliche Messwerte unterhalb des Grenzwertes der Luftkapazität von 5 Vol.\%. Für den Sonderbereich GEO weisen darüber hinaus zwölf Messungen eine gesättigte Wasserleitfähigkeit $<10 \mathrm{~cm} / \mathrm{d}$ aus. Eine derartige Konzentration von Einzelmessungen im dritten Quadranten kann bei der Variante 7,5Mg@350kPa 
nicht festgestellt werden: hier bewegen sich die Messwerte der Wasserleitfähigkeit um den Grenzwert. 75\% der Messwerte liegen oberhalb des Grenzwertes, also im zweiten Quadranten, nur ein Viertel befindet sich im dritten Quadranten.

Im Bereich des Unterbodens (60 - $64 \mathrm{~cm}$ ) liegen die Einzelmessungen zwar bei allen Varianten im dritten Quadranten, die Verteilungen der Messwerte unterscheiden sich jedoch voneinander. So weisen die Daten der Kontrollparzelle eine große Streuung ihrer Wasserleitfähigkeit aus, nur zwei der Einzelmessungen unterschreiten den Grenzwert. In Bezug auf die Luftkapazität liegen $65 \%$ der Messwerte unterhalb des Grenzwertes von 5 Vol.-\%, unterschreiten diesen in aller Regel jedoch nur geringfügig, so dass sich das Gros der Werte entlang der eingezeichneten Grenzwertlinie befindet. Im Sonderbereich GEO unterschreiten sechs der Messwerte den Grenzwert der Luftkapazität und zwei jenen der Wasserleitfähigkeit, jedoch streuen die Werte weniger stark und liegen zumeist nahe dem Schnittpunkt der Grenzwert-Linien. Eine stärkere Streuung der Werte ist bei Variante 7,5Mg@350kPa zu beobachten: obwohl sie jener der Kontrolle gleicht, finden sich hier mehr Einzelmessungen, die z. T. deutlich höhere Luftkapazitäten aufweisen als das Gros der Proben. Unter Berücksichtigung der protokollierten Regenwurmgänge in den Bodenproben ergibt sich ein der Kontrollvariante sehr ähnliches Bild mit einer breiten Streuung der Wasserleitfähigkeit oberhalb des Grenzwertes und Messwerten für die Luftkapazität, die in 55\% der Fälle den Grenzwert unterschreiten, jedoch nur in drei Fällen $<4$ Vol.-\% betragen.

Im Rahmen der Spatendiagnose der beprobten Bereiche wurden die Tiefen Krume $(0-20 \mathrm{~cm})$, Krumenbasis $(20-35 \mathrm{~cm})$ und Unterboden $(35-50 \mathrm{~cm})$ untersucht. Die Ergebnisse der Untersuchungen sind in Tabelle 9 dargestellt, jene der erweiterten Spatendiagnose sind Tabelle A 49 zu entnehmen. 
Tabelle 9: Ergebnisse der Spatendiagnose vom 06.10.2008 auf der Fläche $\mathbf{N R W}_{\text {konv }}$ (nach DBG 2005)

\begin{tabular}{|c|c|c|c|c|c|c|}
\hline $\begin{array}{l}\text { Variante/ } \\
\text { Schicht }\end{array}$ & Aggregatgröße & $\begin{array}{l}\text { Verfesti } \\
\text { gungs- } \\
\text { grad }\end{array}$ & $\begin{array}{l}\text { Lagerungs- } \\
\text { art }\end{array}$ & $\begin{array}{l}\text { Makro- } \\
\text { poren- } \\
\text { anteil } \\
\end{array}$ & $\begin{array}{l}\text { Wurzel- } \\
\text { verteilung }\end{array}$ & $\begin{array}{l}\text { Pack- } \\
\text { ungs- } \\
\text { dichte }\end{array}$ \\
\hline \multicolumn{7}{|c|}{ 7,5Mg@350kPa } \\
\hline Krume & Krümelgefüge & Vf1 & offen & gri1-2 & W4 & $\mathrm{Pd} 1$ \\
\hline $0-20 \mathrm{~cm}$ & $1-2 \mathrm{~cm}$ & & & $\mathrm{f} 4$ & $(\mathrm{Wg} 1, \mathrm{Wf} 4)$ & \\
\hline Krumenbasis & Bröckelgefüge & Vf2 & geschlossen & gri1-2 & W4 & $\mathrm{Pd} 2$ \\
\hline $20-35 \mathrm{~cm}$ & $>5 \mathrm{~cm}$ & & & $\mathrm{f} 4$ & $(\mathrm{Wg} 1, \mathrm{Wf} 4)$ & \\
\hline Unterboden & Sub-polyeder & Vf3 & geschlossen & gri2 & W2 & $\mathrm{Pd} 3$ \\
\hline $35-50 \mathrm{~cm}$ & $>5 \mathrm{~cm}$ & & & $\mathrm{f} 4$ & $(\mathrm{Wg} 0, \mathrm{Wf2})$ & \\
\hline Krume & Krümelgefüge & Vf1 & offen & gri2 & W4 & $\mathrm{Pd} 1$ \\
\hline $0-10 \mathrm{~cm}$ & $1-2 \mathrm{~cm}$ & & & $\mathrm{f} 4$ & $(\mathrm{Wg} 1, \mathrm{Wf} 4)$ & \\
\hline Krumenbasis & $\begin{array}{l}\text { Bröckel- bis } \\
\text { Klumpen } \\
\text { gefüge }\end{array}$ & Vf3 & geschlossen & gri1 & W3 & $\mathrm{Pd} 3$ \\
\hline $10-35 \mathrm{~cm}$ & $>5 />10 \mathrm{~cm}$ & & & $\mathrm{f} 2$ & $(\mathrm{Wg} 1, \mathrm{Wf3})$ & \\
\hline Unterboden & Sub-polyeder & Vf3 & geschlossen & gri1 & W1 & $\mathrm{Pd} 4$ \\
\hline $35-50 \mathrm{~cm}$ & $>5 \mathrm{~cm}$ & & & f3 & $(\mathrm{Wg} 0, \mathrm{Wf1})$ & \\
\hline \multicolumn{7}{|l|}{ Geo } \\
\hline Krume & Krümelgefüge & Vf1 & offen & gri2 & W4 & $\mathrm{Pd} 1$ \\
\hline $0-10 \mathrm{~cm}$ & $1-2 \mathrm{~cm}$ & & & $\mathrm{f} 4$ & $(\mathrm{Wg} 1, \mathrm{Wf} 4)$ & \\
\hline Krumenbasis & Bröckelgefüge & Vf2 & geschlossen & gri2 & W3 & $\mathrm{Pd} 3$ \\
\hline $10-35 \mathrm{~cm}$ & $>5 \mathrm{~cm}$ & & & f2 & $(\mathrm{Wg} 1, \mathrm{Wf3})$ & \\
\hline Unterboden & Sub-polyeder & Vf3 & geschlossen & gri1 & W1 & Pd3-4 \\
\hline $35-50 \mathrm{~cm}$ & $>5 \mathrm{~cm}$ & & & $\mathrm{f} 4$ & $(\mathrm{Wg} 0, \mathrm{Wf1})$ & \\
\hline
\end{tabular}

Bei allen drei Varianten kann in der Krume ein Bodengefüge aus offen gelagerten porösen Krümeln mit einer Größe von 1 bis $2 \mathrm{~cm}$ beobachtet werden. Der Verfestigungsgrad ist jeweils gering mit einem hohen Anteil an 
Makroporen, der in der Kontrolle am höchsten bewertet wird. Packungsdichte, Wurzelverteilung und die Verteilung der Ernterückstände werden in der Krume in allen Bereichen als günstig bewertet und weisen keinerlei Unterschiede zueinander auf.

Im Bereich der Krumenbasis weisen die befahrenen Bereiche einen höheren Verfestigungsgrad gegenüber der Kontrolle auf, außerdem eine höhere Packungsdichte und eine ungünstigere Wurzelverteilung. Darüber hinaus wird in der Variante 7,5Mg@350kPa das Bodengefüge nicht mehr als Bröckelgefüge, wie bei den anderen beiden Varianten, sondern bereits als Übergang zum Subpolyedergefüge mit Aggregatgrößen $>10 \mathrm{~cm}$ angesprochen. Im Bereich GEO können Ansätze zu einem plattig gelagerten Gefüge identifiziert werden.

Im Bereich des Unterbodens weisen die befahrenen Varianten die höheren Packungsdichten und eine ungünstigere Wurzelverteilung aus als die Kontrolle, wohingegen sich Aggregatgröße und Verfestigungsgrad nicht unterscheiden. Die Verteilung der Ernterückstände ist in allen Parzellen in einer Tiefe von 30 $40 \mathrm{~cm}$ als ungünstig zu bewerten. Die Durchwurzelung der befahrenen Parzellen fällt negativ aus, da insbesondere in einem Bereich ab $35 \mathrm{~cm}$ Bodentiefe nur noch einzelne Wurzelgänge zu identifizieren sind. 


\section{Diskussion}

Vorrangige Ziele der vorliegenden Arbeit war die Entwicklung eines Horizontalpenetrometers, sein Einsatz im Rahmen eines Feldversuchs und eine Bewertung des entwickelten Systems und der gewonnen Daten anhand weiterer Untersuchungsergebnisse und Literaturangaben.

Das an der FH-SWF entwickelte Messsystem TASIS verfügt über prismenförmige Messsonden und erfasst den horizontalen Eindringwiderstand in drei Messtiefen (5 - $14 \mathrm{~cm}, 18-27 \mathrm{~cm}, 30-39 \mathrm{~cm})$. TASIS wurde über einen Versuchszeitraum von drei Jahren (2006-2008) in einer großflächigen Versuchsanordnung eingesetzt, wobei an zwei Standorten (SH, NRW) die Einflüsse der Parameter Bodenbearbeitung (konventionell, konservierend), Radlast (3,3 Mg, 6,3 Mg, 7,5 Mg) und Reifeninnendruck (hoch bzw. niedrig für jede Radlastvariante) auf den Eindringwiderstand untersucht wurden.

Unter den gegebenen Standort- und Versuchsbedingungen konnte TASIS auf sandigen Böden mit unterschiedlich starkem Lehmanteil und auf den schluffigen Lössböden der Soester Börde eingesetzt werden. Die Sandböden in der schleswig-holsteinischen Jungmoränenlandschaft erwiesen sich hierbei aufgrund ihres hohen Anteils an Grobboden (Bodenskelett), also Teilchen mit einem Äquivalentdurchmesser $>2 \mathrm{~mm}$, als anspruchsvoll für Penetrometermessungen. Insbesondere Messungen mit dem handgeführten Vertikalpenetrometer wurden durch den hohen Besatz an Steinen und Findlingen erschwert. Zahlreiche Messungen mussten vor dem Erreichen der angestrebten $80 \mathrm{~cm}$ Messtiefe abgebrochen werden. Beim Einsatz von TASIS sprachen auf den schleswig-holsteinischen Versuchsflächen häufiger die Scherbolzen an und Messfahrten mussten unterbrochen werden. Die nordrheinwestfälischen Lössstandorte waren aufgrund ihrer fast vollständigen Steinfreiheit für die Anwendbarkeit des Messverfahrens Penetrometer günstiger. Schluffige oder fein-lehmige Böden wurden auch von anderen Autoren für den Einsatz von Horizontalpenetrometern gewählt (u. a. HALL \& RAPER 2005, Sharifi ET AL. 2007, Chung et AL. 2004, SiEFKEN ET AL. 2005, SuN 
ET AL. 2006) oder Tests wurden in homogenisierten Böden unter Laborbedingungen durchgeführt (CHUKWU \& BOWERS 2005). Bei SCHWARK (2005), der im Zuge seiner Untersuchungen mit dem Horizontalpenetrometer auf 34 Schlägen in Schleswig-Holstein ebenfalls Erfahrungen mit Steinbesatz gesammelt haben muss, finden sich keine Angaben $z u$ möglichen Beeinträchtigungen.

Günstig für den Standort NRW ist weiterhin zu werten, dass sich beide Versuchsflächen in unmittelbarer Nähe zueinander befanden und - bis auf die Bodenbearbeitung - eine synchrone Bewirtschaftung durchgeführt wurde. Dieses war am Standort $\mathrm{SH}$ nicht der Fall und so konnten sich einzelne Termine stark unterschiedliche Voraussetzungen für eine Messwertaufnahme finden. Im Herbst 2006 nahm die mehr als einjährige Kleegrasnarbe einen großen Einfluss auf die Höhe der gemessenen vertikalen EW (vgl. Abbildung 20). Der Wurzelfilz des Kleegrases erschwerte zudem die Datenaufnahme von TASIS, da die Schare Filz und Erdanhang aufschoben und das System aufgrund nebeneinander montierter Schare zum Stopfen neigte. Die unterschiedlichen Bewirtschaftungsweisen der Versuchsgüter Lindhof $\left(\mathrm{SH}_{\text {konv, }}\right.$ ökologische Produktion) und Hohenschulen ( $\mathrm{SH}_{\mathrm{kons}}$, konventionelle Produktion) werden als nicht relevant im Sinne der Versuchsfragen angesehen. Diese Einschätzung findet sich auch bei SCHWARK (2005).

Die Parameter des technogenen Lasteintrages waren wie beschrieben gewählt, um Ergebnisse mit praxisrelevantem Bezug zu produzieren. Sämtliche Befahrungen im Feldversuch wurden mittels des Lastrahmens Movis durchgeführt, wodurch sichergestellt werden konnte, dass Fahrwerkskonstellationen simuliert werden, wie sie in der landwirtschaftlichen Praxis vorzufinden sind. Die mechanische Auflast konnte ebenso gezielt angepasst werden, wie der Reifeninnendruck. Der verwendete Reifen entsprach dem aktuellen technischen Stand und wurde gemäß Herstellerangaben eingesetzt. Geäußerte Kritik an der Durchführung anderer Untersuchungen (HALVORSON ET AL. 2003; MÄHNER 1999; PYTKA ET AL. 2006) zum Thema Bodenverdichtung wurde wurde hiermit Rechnung getragen. 
Die Zeitpunkte der Befahrungsversuche repräsentieren eine schlechte landwirtschaftliche Praxis und befinden sich in einem bewussten Widerspruch zu den Vorgaben einer guten fachlichen Praxis (BMVEL 2002) Dieses Vorgehen deckt sich mit Bedingungen, die bei Untersuchungen von SCHÄFERLANDEFELD ET AL. (2004); HEUER ET AL. (2008), andere Studien hingegen wurden unter trockeneren Bedingungen durchgeführt (Z. B. CHUNG ET AL. 2004; CHUKWU \& BOWERS 2005; KELLER ET AL. 2002). Eine Befahrung bei hohen Bodenwassergehalten war gemäß des Versuchszieles, Bodenveränderungen durch mechanische Auflasten zu erzeugen und mit Hilfe eines Penetrometers nachzuweisen, legitim, deckt sich aber nicht mit entsprechenden Handlungsempfehlungen, die ein bodenschonendes Befahren vorsehen (BRANDHUBER ET AL. 2008; MUNLV 2004; VDI 2007).

Die Abstufung der Versuchsfaktoren Radlast und Reifeninnendruck können als vorteilhaft bewertet werden, da sie die getrennte Betrachtung der Einflüsse beider Faktoren auf den untersuchten Parameter Eindringwiderstand ermöglichten. Die Abstufung der Radlasten ergab sich dabei aus der zu Versuchsbeginn möglichen minimalen Radlastvariante (Movis unballastiert, 3,2 Mg) und der im Frühjahr 2006 maximalen Auflastvariante (Movis ballastiert, 6,2 Mg). Erst im Herbst 2006 konnte durch technische Umbaumaßnahmen eine weitere Erhöhung der Radlast auf 7,5 Mg realisiert werden. Die Varianten mit mittlerer und geringer Radlast wurden - mit geringen Änderungen durch erfolgte Umbaumaßnahmen - beibehalten. Trotz der ungleichen RadlastFaktorstufen konnte bei den TASIS-Messungen auf NRW $_{\text {konv }}$ ein gut abgestuftes Bild der Varianten bezogen auf den EW ermittelt werden (Abbildung 28 und Abbildung 29). Für die Analyse des Einflusses von Radlast und Reifeninnendruck wäre aber u. U. ein weiteres Abstufungsverhältnis vorteilhaft gewesen.

Das geordnete Versuchsdesign stellte hohe Ansprüche an die Fähigkeit der einzelnen Projektpartner, Flächen in ausreichender Größe über einen Zeitraum von knapp 3 Jahren zur Verfügung zu stellen. Es bot sich nach der Einführung einer dritten Radlastvariante im Herbst 2006 nicht auf allen Flächen die Möglichkeit, eine vollständige Wiederholung in drei Blöcken zu fahren und die Versuchsanordnung musste in $\mathrm{SH}$ und auf $\mathrm{NRW}_{\text {kons }}$ auf zwei Blöcke reduziert 
werden. Ex post wäre eine Reduzierung der Anzahl der Belastungsvarianten zugunsten einer dritten Wiederholung oder des Faktors Überrollhäufigkeit, wie er von ZINK (2009) in Bodengrubenuntersuchungen angestellt wurde, eine sinnvolle Alternative gewesen.

Die errechneten Kontaktflächen weisen eine extrem große Streuung auf, die im wesentlichen von der bearbeitungs- und witterungsbedingten Oberbodenbeschaffenheit bei Befahrung und des weiteren durch die erschwerte Datenauswertung der Bilddateien am PC aufgrund des Pflanzenbewuchses (Kleegras, Sommerhafer) beeinträchtigt wurde (vgl. Tabelle 4). Die durchschnittlich niedrigeren Kontaktflächen auf den Geschiebemergeln Schleswig-Holsteins bedingen sich durch die höhere Lagerungsdichten, die von ZINK (2009) für diesen Standort gemessen wurden $(+9,2 \%$ gegenüber NRW). Sie reduzieren die Einsinktiefe des Reifens. Diese Werte korrelieren größenordnungsmäßig mit der relativ niedrigeren Kontaktfläche am Standort SH $(-7,5 \%)$.

Die Tatsache, dass die konservierend bewirtschafteten Flächen im Durchschnitt um gut $20 \%$ höhere Kontaktflächen gegenüber den wendend bewirtschafteten Flächen aufweisen, erstaunt, wäre doch zu vermuten gewesen, dass auf konventionell bewirtschafteten Flächen aufgrund der intensiven Lockerung und der einhergehenden Strukturzerstörung durch den Pflugeinsatz die mechanische Stabilität gegenüber Auflasten verringert und ein entsprechend tieferes Einsinken des Reifens die Folge ist. Es erstaunt weiterhin, weil die gemessenen Spurtiefen für die konservierend bewirtschafteten Flächen die niedrigeren Spurtiefen aufweisen. Nach BRUNOTTE ET AL. (2001) lagern konservierend bewirtschaftete Böden insgesamt dichter, was auch die Daten für die untersuchten Flächen bestätigen. Die Strukturarmut der intensiv bewirtschafteten Flächen mag jedoch ein Grund dafür sein, dass durch Verschlämmung und oberflächliche Abtrocknung die Einsinktiefe im angewandten statischen Messverfahren vermindert wird, während sie gleichzeitig bei einer dynamischen Belastung (Befahrung) dazu beiträgt, dass ein verstärktes Aufschieben des Bodens vor dem Reifen („Bulldozing“) erfolgt 
und in einer tieferen Spurbildung resultiert. ZINK (2009) beschreibt für die konservierend bewirtschafteten Versuchsflächen strukturiertere und stabilere Oberböden, die es vermögen, mechanische Auflasten besser in die horizontale Ebene abzustützen. Hieraus würde ein geringeres Verschieben der Bodenteilchen durch den Reifen und damit geringere Spurtiefen resultieren.

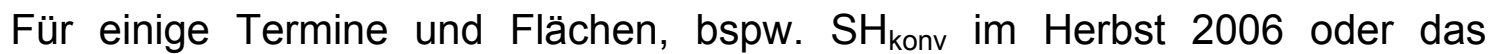
Frühjahr 2007, ergaben sich aufgrund der trockenen Bedingungen sehr geringe Kontaktflächen und sehr hohe Kontaktflächendrücken von teilw. über $500 \mathrm{kPa}$. Der Reifen stützte sich auch in den schwerlastigen Varianten ausschließlich auf den Stollen ab und nicht auf den Stollenzwischenraum, da die resultierende Einsinktiefe bei den vorherrschenden Bodenverhältnissen geringer war als die mit $59 \mathrm{~mm}$ angegebene Profiltiefe (vgl. MICHELIN 2006). Die Reifenaufstandsfläche setzte sich entsprechend aus Einzelflächen zusammen, die bei der Berechnung aufaddiert wurden. Über das Modell des Kontaktflächendrucks, also der Gewichtskraft bezogen auf die Reifenkontaktfläche mit dem Boden, ergeben sich entsprechend hohe Flächendrücke. Dieses ist im Modell des Kontaktflächendruckes nicht berücksichtigt, weil ein homogener Lasteintrag über die Fläche unterstellt wird (PETH \& HORN 2004). Dennoch bestätigen Keller \& ARVIDSSON (2004) die guten Eigenschaften des Modells, um den vertikalen Spannungseintrag zu prognostizieren. Die Grenzen des Modells hatte jedoch schon Söhne selbst erkannt, der schrieb: „Je nach Größe der Belastungsflächen und der Bodenart kann die Flächenpressung an der Oberfläche von dem Mittelwert [...] erheblich abweichen (SÖHNE 1951)." GYSI ET AL. (2001) konnten an Untersuchungen mit fünf verschiedenen landwirtschaftlichen Reifen zeigen, dass Drücke direkt unterhalb des Reifens nicht gleichförmig verteilt sind, sondern die höchsten gemessenen Drücke, die in den Randbereichen eines Reifens gemessen werden, um den Faktor 4 - 10 gegenüber den mittleren Kontaktflächendrücken erhöht sein können. Auch HAMmel (1994) stellte fest, dass Reifen, die sich ausschließlich auf die Stollen abstützen sehr heterogene Spannungen direkt unterhalb des Reifens erzeugen, die über die Tiefe abnehmen. In der Untersuchung von GYSI ET AL. (2001) konnten die Spitzendrücke durch eine $30 \mathrm{~cm}$ dicken Sandauflage bei pF 1,8 signifikant reduziert werden und nahmen 
in dieser Tiefe von der Reifenmitte nach außen degressiv ab. Auch JoHnson \& BURT (1990) weisen auf die Einschränkungen eines gemittelten Flächendruckes hin und stellen eigene Berechnungsmodelle vor.

Dieses erfährt eine besondere Bedeutung bei der Wahl des Einstichpunktes für die Vertikalpenetrometermessungen, der bei der vorliegenden Arbeit im Bereich des Stollenabdrucks im zentralen Bereich der Reifenaufstandsfläche gewählt wurde. Nach den Ergebnissen von GYSI ET AL. (2001) kann also vermutet werden, dass Messungen des vertikalen EW unter trockenen Bodenverhältnissen die Verdichtungswirkung der mechanischen Belastung in den oberen Bodenschichten unterschätzen, da der zentrale Bereich relativ geringer belastet wird. Die Umkehr dieses Verhältnisses über die Tiefe rechtfertigt jedoch die Wahl des Einstichpunktes, da in tiefer liegenden Schichten ein maximaler Spannungeeintrag im zentralen Bereich der Fahrspur zu unterstellen ist.

Die Vertikalpenetrometer (VP) - Messungen lieferten in Bezug auf die Flächencharakterisierung Aussagen über die Lage und Ausprägung von Verdichtungszonen auf den Versuchsflächen (vgl. Abbildung 15). Die Daten bilden die Bodenschichtencharakteristik der einzelnen Flächen in ihrer Bearbeitungshistorie gut ab: Pflugeinsatz und langjährig konservierende BB lassen sich klar von einander differenzieren und zumindest beim Vergleich der konventionell bewirtschafteten Flächen ist zu vermuten, dass auf den Lössstandorten die BB tiefer erfolgt ist als die angegebenen $28 \mathrm{~cm}$, mit einer ausgeprägten Pflugsohle im Bereich $35-45 \mathrm{~cm}$. Auf $\mathrm{SH}_{\text {kons }}$ liegt dieser Bereich ca. $10 \mathrm{~cm}$ höher. Auch Gorucu ET AL. (2006) und DesBiolles ET AL. (1999) nutzten Daten aus VP-Messungen und leiteten aus ihren Daten die optimale Bearbeitungstiefe bzw. den erforderlichen Zugkraftbedarf für die Bodenbearbeitung ab.

Das Niveau der aufgenommenen VP-Daten deckt sich größenordnungsmäßig mit denen anderer Arbeiten (To \& KAY 2005, WHALLEY ET AL. 2007, UNGER \& JONES 1998) auch wenn teilweise Penetrometer nach dem ASAE-Standard $\left(30^{\circ}\right.$ Öffnungswinkel) Anwendungen fanden. Auch vergleichbar hohe 
Standardabweichungen finden sich bei anderen Autoren. LAPEN ET AL. (2004) betonen daher, dass der EW ein hochgradig variabler Indikator der Bodendichte ist.

Im Vergleich mit den von ZINK (2009) errechneten Daten der horizontspezifischen Vorbelastungswerte zeigt sich, dass diese nur bedingt mit den VPDaten übereinstimmen: Auf $\mathrm{NRW}_{\text {kons }}$ zeigen die Vorbelastungswerte im AISWHorizont (28-45 cm) erhöhte Werte gegenüber darunter und darüber befindlichen Horizonten was auf eine intensivere mechanische Belastung und damit für eine höhere Stabilität spricht und sich somit in erhöhten vEW ausdrücken müsste. Die Vertikalpenetrometermessungen zeigen aber in einer Bodentiefe $>30 \mathrm{~cm}$ abnehmende vEW bis in eine Tiefe von etwa $55 \mathrm{~cm}$. Auch

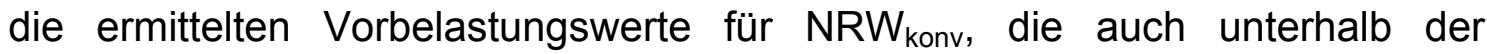
Pflugsohle stetig steigende Werte aufweisen, decken sich nicht mit der auf der Fläche gemessenen Abnahme der hEW nach dem Durchstoßen dieses Horizontes.

Werden die Werte der Lagerungsdichten und Bodenwassergehalte betrachtet, so sind Einflüsse beider Faktoren auf den vEW erkennbar. Auf $\mathrm{SH}_{\text {kons }}$ reagiert der $\mathrm{hEW}$ im Bereich bis $40 \mathrm{~cm}$ mit einem Anstieg von ca. $1 \mathrm{MPa}$ auf steigende Lagerungsdichten um $0,25 \mathrm{~g} / \mathrm{cm}^{3}$ bei nahezu konstantem Bodenwassergehalt. HENDERSON ET AL. (1988) konnten zwar in Untersuchungen auf Sandböden feststellen, dass der vEW nur geringfügig vom Wassergehalt beeinflusst wird, wenn dieser $70 \%$ der Feldkapazität oder mehr beträgt. Der Einfluss und die Bedeutung des Bodenwassergehaltes auf den EW ist jedoch unumstritten: DEXTER ET AL. (2007) haben eine Formel entwickelt, die unter Addition des Grades der Bodendichte („degree of compactness“) und des aus dem Porenwasserdruck resultierenden effektiven Stress eine Vorhersage über den EW treffen kann. Auch WhalleY ET AL. (2007) konnten zeigen, dass Lagerungsdichte und Bodenwassergehalt die tragenden Rechengrößen bei der Vorhersage des EW sind. LAPEN ET AL. (2004) konnten unter verschiedenen Vegetations- und Befahreinflüssen feststellen, dass zwischen Bodenwassergehalt und EW eine sehr enger Zusammenhang besteht, der sich über die Vegetationsperiode aber unterschiedlich stark ausprägen kann. Die Autoren weisen aber ausdrücklich auf die Komplexität der Zusammenhänge 
hin, die das Wassergehalt-Einringwiderstands-Verhältnis beeinflussen. In der vorliegenden Arbeit drückt sich der Einfluss des Bodenwassergehaltes im höheren Messwertniveau aus der schleswig-holsteinischen Flächen aus, die eine durchschnittlich höhere Wasserspannung aufweisen. In $40 \mathrm{~cm}$ Tiefe liegen die Wasserspannungen der konservierend bewirtschafteten Flächen bei > 98\% FK, während sich jene der konventionellen Flächen mit 100\% FK (NRW) und $81 \%$ FK deutlicher unterscheiden. Die um $+44 \%$ höheren vEW von $\mathrm{SH}_{\text {kons }}$ gegenüber $N R W_{k o n s}$ in der Tiefe $30-39 \mathrm{~cm}$ kann somit den Bodeneigenschaften zugeschrieben werden, während die um $+173 \%$ erhöhten vEW der konventionellen Flächen neben den Bodenunterschieden auch auf einen unterschiedlichen Wassergehalt zurückgeführt werden müssen. Weiterhin gilt es zu berücksichtigen, dass der Messtiefenbereich $30-39 \mathrm{~cm}$ auf $N_{R W} W_{k o n v}$ geringfügig oberhalb der Pflugsohle liegt, während er sich auf $\mathrm{SH}_{\text {konv }}$ leicht darunter befindet. Auch dadurch bedingt sich ein höheres Messwertniveau am schleswig-holsteinischen Standort.

Der Einfluss des Bodenwassergehaltes bzw. der von inm induzierten Prozesse (Quellung, Schrumpfung, etc.) finden Ausdruck in den VP-Messungen des Herbstes 2006. Ein Starkregenereignis zwischen den VP-Messungen vor und nach Befahrung nahm hier einen starken Einfluss auf die Messungen. Auf $\mathrm{SH}_{\text {kons }}$ führten steigende Bodenwassergehalte zu sinkenden vEW trotz erfolgter Belastung. Auf $\mathrm{SH}_{\text {konv }}$ ist eine solche Gesetzmäßigkeit nicht zu erkennen, was sich auf drei Gründe zurückführen lässt: zum einen war die gefallene Niederschlagsmenge mit $32,6 \mathrm{~mm}$ niedriger als auf dem Versuchsgut Hohenschulen ( $\mathrm{SH}_{\mathrm{kons}}, 40,3 \mathrm{~mm}$ ). Zum zweiten liegen die vertikalen gesättigten Wasserleitfähigkeiten auf $\mathrm{SH}_{\text {konv }}$ auf einem höheren Niveau $(57-126 \mathrm{~cm} / \mathrm{d})$ und der gefallene Niederschlag war zum Zeitpunkt der zweiten Messung bereits vollständig durch den Boden hindurch gelaufen. Auf $\mathrm{SH}_{\text {kons }}$ wurde die Feuchtigkeit aufgrund der höheren Menge und der geringeren Leitfähigkeiten stärker zurückgehalten und die VP-Messungen entsprechend beeinflusst. Zum dritten wirkt sich der Wassergehalt auch die Druckausbreitung im Boden aus, da sich in stark aufgesättigten Böden bei kurzzeitiger dynamischer Belastung zunächst der Porenwasserdruck erhöht, und eine Dichtlagerung der Bodenteilchen erst stattfindet, wenn das Porenwasser (bei längerer Belastung) 
abfließt (vgl. DISERENS \& BUCHER 1997; HORN 2004). Erst dann wäre auch ein Effekt mit dem Penetrometer nachweisbar.

Effekte in Bezug auf die Verdichtungswirkung mechanischer Belastungen auf den vEW konnten deutlich in den oberen Bodenschichten identifiziert werden, wenn der Boden zuvor wendend gelockert worden war (vgl. 4.4.2). Unter diesen Bedingungen war auch eine Differenzierung der mechanischen Belastung möglich. Ab einer Messtiefe von $30 \mathrm{~cm}$ und damit im Bereich der Pflugsohle bzw. unterhalb des Bearbeitungshorizontes waren nur noch unscharfe Effekte einer Befahrung auf den vEW erkennbar.

Abgestufte Effekte von mechanischen Lasteinträgen, wie sie das Versuchsdesign berücksichtigt hat, waren mit der Methode Vertikalpenetrometer nur schwer zu greifen und einzuordnen (vgl. Tabelle 5). Auf den nordrhein-westfälischen Versuchsflächen lassen die Untersuchungen - auch aufgrund der oben angesprochenen Bodeneigenschaften - bessere Ergebnisse erkennen. Auf beiden Flächen und nur mit Ausnahme des Frühjahres 2008 können Effekte eines dynamischen Lasteintrages auf den vEW festgestellt werden. Diese bleiben aber in ihrer Aussagekraft aufgrund der von LAPEN ET AL. (2004) angesprochenen Varianz eingeschränkt. Effekte eines angepassten Reifeninnendruckes auf den vEW bewegen sich im Bereich des Datenrauschens und sind in den Datensätzen nicht zu identifizieren. Unter für das Verfahren günstigen Bedingungen (geringer Grobbodenanteil, homogene Bodenstruktur, wendende BB) sind Effekte einer Radlasterhöhung von 3,3 Mg auf 6,3 Mg zu erkennen, aber nicht statistisch zu belegen (vgl. Abbildung 21). Sie bleiben aber auf den Bereich oberhalb der Pflugsohle beschränkt. Die von ZINK (2009) ermittelten Spannungseinträge bis in $60 \mathrm{~cm}$ Bodentiefe können mit der Methode auch unter günstigen Bedingungen nicht nachgewiesen werden. Der Anteil der plastischen Bodendeformation beträgt in $40 \mathrm{~cm}$ Tiefe auch bei hohen Radlasten von 7,5 Mg weniger als $3 \mathrm{~mm}$ - dieses kann die Methode VP nicht mehr abbilden. Plastische Deformationen in $20 \mathrm{~cm}$ Tiefe betragen für die hohen Radlastvarianten zwischen $11 \mathrm{~mm}$ und $14 \mathrm{~mm}$ und scheinen damit eine kritische Grenze überschritten zu haben, die unter günstigen Bedingungen einen Nachweis dieser Deformation mit dem VP ermöglichen. Auch GYSI ET AL. 
(1999) konnten mit dem VP keine Aussagen bzgl. einer Verdichtungswirkung treffen und mussten die gewonnenen Daten als nicht-erklärbar einstufen.

Mehr als für die Erfassung kleinräumiger Änderungen der Bodenverhältnisse scheint die Methode jedoch geeignet zu sein, ein langfristiges Monitoring von Flächen in Bezug auf Lage und Mächtigkeit von Bearbeitungshorizonten zu gewährleisten. Hierfür ist es jedoch erforderlich, dass die Messungen zu vergleichbaren Zeitpunkten durchgeführt werden.

Das Horizontalpenetrometer-Systems TASIS hat sich in seinem konstruktiven Ansatz unter den gegebenen Bedingungen im Feldeinsatz bewährt. Die oben aufgeführten Probleme stark steiniger Böden haben naturgemäß Auswirkungen auf Qualität und Umfang der erhobenen Daten, konstruktiv konnte aber auch Steinbesatz in Findlingsgröße keine physische Beeinträchtigungen an TASIS hervorrufen. Der konstruktive Ansatz eines drehbar gelagerten inneren Scharkörpers, der durch die starre Ummantelung eines äußeren Schares vor mechanischen Einflüssen geschützt wird, ermöglichte auch unter den schwierigen Bedingungen schleswig-holsteinischer Endmoränenstandorte eine zuverlässige Datenaufnahme. Bei starken mechanischen Beanspruchungen, etwa durch das Auftreffen auf einen großen Stein, sprach die ScherbolzenSicherung an und sowohl Schar wie auch Kraftaufnehmer blieben unbeschadet. Die räumlich getrennte Positionierung der gesamten Messtechnik vom eigentlichen Ort der Messwertaufnahme bot weiterhin den Vorteil, dass eine Sichtprüfung der einzelnen Komponenten auf evtl. mechanische Beschädigungen jederzeit und ohne Werkzeug möglich war. Die Messelemente waren auf maximale Kraftaufnahme von $5 \mathrm{kN}, 10 \mathrm{kN}$ und $20 \mathrm{kN}$ geeicht, so dass eine flächenindividuelle und zügige Anpassung der Messtechnik vor Ort an die jeweiligen Bedingungen vorgenommen werden konnte. Problematisch gestaltete sich jedoch die bauliche Nähe $(\mathrm{ca} .30 \mathrm{~cm})$ der einzelnen Schare zueinander wenn ein starker Vegetationswuchs zum Zeitpunkt der Datenaufnahme vorherschte. Bereits im Herbst 2006 waren die Messungen auf $\mathrm{SH}_{\mathrm{konv}}$ durch den Wurzelfilz des Kleegrases beeinträchtigt worden. Hierauf hin wurden die äußeren Scharkörper traktorseitig auf $45^{\circ}$ abgeschliffen, um ein 
leichteres Durchtrennen von Vegetationsdecken zu ermöglichen. Ein identischer Ansatz findet sich bei SIEFKEN ET AL. (2005), die Untersuchungen von GiLL \& VANDEN BeRG (1967) zitieren, nach denen Winkel von 40 - $45^{\circ}$ eine minimale Durchzugskraft („cutting force") erfordern. Alternative Überlegungen, die die Platzierung einer Sech-Scheibe vor dem Schar vorsahen, wurden aufgrund der konstruktiven Unvereinbarkeit mit der Scherbolzensicherung verworfen. Ein Ansprechen der Sicherung bei Überlast und einem damit verbundenen Wegschwenken des gesamten Scharkörpers inklusive des Seches hätte sich nicht umsetzen lassen. Der wesentliche messtechnische Grund, der gegen eine Sech-Lösung sprach, war die mögliche Beeinflussung der Datenqualität insbesondere in oberflächennahen Messtiefen durch Bodeneingriff und Spannungseintrag des Seches. Bei fast kniehohem Bewuchs von Ausfallgetreide auf $\mathrm{SH}_{\text {konv }}$ in 2007 reichte die gefundene Lösung jedoch nicht mehr aus und es wurde auf eine Messwertaufnahme in $30-39 \mathrm{~cm}$ Messtiefe zugunsten einer gesicherten Datenerfassung in den anderen Bereichen verzichtet.

Nach der Systematik von Hemmat \& AdAMCHUK (2008) werden horizontal arbeitende mechanische Bodensensorsysteme, die mittels einer Sondenspitze den EW erfassen, wie folgt unterteilt - die in Klammern genannten Autoren sind Beispiele für die jeweiligen Systeme:

- Horizontalsensoren mit einer Messspitze (SUn ET AL. 2006, HALL \& RAPER 2005, AliHAmsyah ET AL. 1990)

- Horizontalsensoren mit mehreren Messspitzen (s.u.)

- vertikal oszillierender Sensor (HALL \& RAPER 2005)

TASIS gehört, wie die meisten anderen in Kaptiel 2 beschriebenen Systeme, der zweiten Sensorklasse an. Nach Einschätzung des Autors der vorliegenden Arbeit sollte die Systematik ergänzt werden, indem in Betracht gezogen wird, ob:

a) die Sensoren konusförmig (BÖLENIUS ET AL. 2006, SCHWARK 2005)

prismenförmig (SHARIFI ET AL. 2007, SUN ET AL. 2006, CHUKWU \& BOWERS

2005, GodWIN 2007) oder Hybride (DENKER ET AL. 2007) sind, und 
b) die Sensoren gemeinsam an einem Schar (SIEFKEN ET AL. 2005, GodWIN 2007) oder an separaten Scharen verbaut sind.

TASIS ist aktuell das einzige Sensorsystem, das eine Hybridform als Sondenspitze nutzt. Da im Gegensatz zu vertikal geführten Penetrometern für Horizontalpenetrometer keine entsprechende Normen vorliegen, orientieren sich die eingesetzten Sondenspitzen mehr oder weniger stark an den gültigen Standards zu vertikalen Messwertaufnahme.

Für eine Hybridform spricht die Erfassung der Bodenspannung in vertikaler Dimension an den konischen Spitzen der Sensoren. Die konstruktiv bedingten Lücken von $2,5 \mathrm{~cm}$ zwischen den einzelnen Messtiefenbereichen, die sich durch die äquidistanten Lochbohrungen am Rahmen von TASIS ergeben, können so "geschlossen" werden, indem auch diese Bodencharakteristika durch die Erfassung einer erweiterten Verdrängungszone Eingang in den integralen Sondenmesswert finden. Zur Druckverteilung um eine vertikal geführte Penetrometerspitze haben GARCIANO ET AL. (2007) Untersuchungen durchgeführt, die zeigten, dass unter Laborbedingungen mehrere Zentimeter von der Sondenmitte Spannungsmaxima auftreten. Die Autoren schätzen jedoch die Zone des Spannungseintrages unter Feldbedingungen als gering ein.

In diesem Zusammenhang ist die auch die festgelegte Wahl der Messtiefenbereiche zu erwähnen, die - wie erwähnt - eine nahezu lückenlose Aufnahme der horizontalen EW bis in $39 \mathrm{~cm}$ Bodentiefe ermöglicht. Wie in Abbildung $15 \mathrm{zu}$ erkennen ist, erfasst die definierte Messtiefe von $30-39 \mathrm{~cm}$ auf den konventionell bewirtschafteten Flächen den Pflugsohlenbereich leicht unter- $\left(\mathrm{SH}_{\text {konv }}\right)$ bzw. oberhalb $\left(\mathrm{SH}_{\text {konv }}\right)$ des Maximums. Eine angepasste Arbeitstiefe an den jeweiligen Standort wäre ex post betrachtet eine sinnvolle Alternative gewesen, dann allerdings mit der Einschränkung, dass eine direkte Vergleichbarkeit der Tiefenwirkung einer mechanischen Belastung eingeschränkt und möglicherweise eine Überlagerung von Messtiefenbereichen die Folge gewesen wären. Eine horizontspezifische Tiefenführung hätte u. U. 
eine bessere Datenqualität in den Bereichen geliefert, die aufgrund einer hohen Vorbelastung in der Lage sind hohe Spannungen aufzunehmen.

Wie Abbildung 23 und Abbildung 24 zeigen, war TASIS aber auch mit den gewählten Einstellungen sehr gut in der Lage, charakteristische horizontal verlaufende Bodenschichten mit erhöhten EW, ähnlich gut zu erfassen und darzustellen wie das VP. Die aufgenommenen Werte decken sich erkennbar mit den VP-Messungen, sind aber ebenso wenig wie diese mit den von ZINK (2009) errechneten Vorbelastungswerten in Übereinstimmung zu bringen. Einschränkend muss erwähnt werden, dass die originären Datensätze des VP über die zentimetergenaue Auflösung der Messwerte über die Tiefe ein konkreteres Bild zeichnen, als es die integralen Messwerte von TASIS vermögen.

Das Niveau der gemessenen VP-Werte liegt um den Faktor 2,26 über denen von TASIS. HALL \& RAPER (2005) ermittelten in ihren Untersuchungen hierfür einen Faktor von 1,52. Auch die von den Autoren beschriebenen geringen Varianzen bei der Erfassung der horizontalen EW können anhand der TASISMessungen nachvollzogen werden. Ein Aspekt, den HALL \& RAPER (2005) untersucht haben, ist Größe und Positionierung der verwendeten Sensorspitze. Eine größere Grundfläche stellte sich hierbei als günstiger in Bezug auf die Datenqualität heraus, so dass ein Sensor mit $2.500 \mathrm{~mm}^{2}$ Grundfläche als der vorteilhafteste unter drei getesteten. Auch dieses ist ein Wert der größenordnungsmäßig mit der $2.806 \mathrm{~mm}^{2}$ großen Sensorgrundfläche von TASIS übereinstimmt.

TASIS wurde aufgrund einer gewünscht breiten Datenbasis (hohe Anzahl an Datenpunkten in einem kleinen Messfenster) mit einer vergleichsweise niedrigen Geschwindigkeit von $0,27 \mathrm{~m} / \mathrm{s}$ eingesetzt. Ein ähnlicher Geschwindigkeitsbereich findet sich bei SHARIFI ET AL. (2007) und CHUKWU \& BOWERS (2005). Einsatzgeschwindigkeiten von TASIS nahe $10 \mathrm{~km} / \mathrm{h}$ wären konstruktionsbedingt möglich gewesen, wären aber nicht im Sinne einer fehlerminimierten Datenaufnahme gewesen. CHUNG ET AL. (2004) setzte sein Penetrometer mit $1,5 \mathrm{~m} / \mathrm{s}$ ein, und stellte gegenüber $0,5 \mathrm{~m} / \mathrm{s}$ fest, dass die Werte nur um durchschnittlich $2 \%$ höher lagen. Im Rahmen der 
Versuchsanstellung kann davon ausgegangen werden, dass eine höhere Geschwindigkeit keine Vorteile bzgl. der Datenqualität geboten hätte. Vielmehr wäre durch sie die Ermittlung der Schnittstellendaten von TASIS und MOVIS erschwert und der Datenumfang verringert worden.

Die im Feldeinsatz von TASIS erhobenen Daten schwanken zwischen den Standorten SH und NRW. Der bereits angesprochene hohe Grobbodenanteil der Jungmoränenstandorte sowie Unterschiede im Bodenwassergehalt haben die Qualität der Datenaufnahme erkennbar beeinflusst. In einzelnen Messtiefen und Jahren sind Ansätze einer Abstufung zum erfolgten Lasteintrag erkennbar (vgl. Abbildung 25 bis Abbildung 26), eine klare Aussage über einen Anstieg des hEW in Bezug auf eine erfolgte Verdichtungswirkung erlauben sie aber nicht. Doch auch bei ZINK (2009) findet sich keine stetige Abstufung der plastischen Verformung in Abhängigkeit des mechanischen Lasteintrags, obwohl sich die Geschiebemergel- und Lössstandorte nicht in Bezug auf ihre Spannungseinträge unterscheiden (ZINK 2009). Die im Rahmen der bodenkundlichen Untersuchungen gemessenen plastischen vertikalen Verformungen unterscheiden sich jedoch erkennbar in Abhängigkeit von der vorgefundenen Bodenart und betragen unter Geschiebemergel in $20 \mathrm{~cm}$ Tiefe durchschnittlich $3,7 \mathrm{~mm}$ und unter Löss durchschnittlich $6,5 \mathrm{~mm}$. Wie auch bei den VPMessungen, so scheint das Mass der plastischen Verformung im Boden eine Orientierung für die Erfassbarkeit der Veränderungen durch TASIS zu sein. Die am Standort SH mittels TASIS aufgenommenen Effekte sind unabhängig von der erfolgten Bodenbearbeitung auf die Messtiefenbereiche bis $18-27 \mathrm{~cm}$ beschränkt, für die plastische Verformungen von 3,7 mm ermittelt worden waren. Für die darunter liegende Tiefe von $40 \mathrm{~cm}$ betrugen die von ZINK (2009) erfassten plastischen Deformationsanteile unter Geschiebemergel $<1 \mathrm{~mm}$, so dass zu unterstellen ist, dass es sich bei den von TASIS in dieser Tiefe erfassten - und für alle Varianten nicht signifikanten - Unterschieden des hEW um Schwankungen aufgrund der Flächenheterogenität handeln muss. Die hEW der einzelnen Varianten unterscheiden sich auf den Geschiebemergelstandorten in allen Messtiefen um einen ähnlichen Betrag, diese Unterschiede fallen aber in $30-39 \mathrm{~cm}$ aufgrund der stärkeren Streuung der Messwerte weniger stark ins Gewicht. 
Unter Löss betragen die plastischen Deformationen in $20 \mathrm{~cm}$ Tiefe $5,5 \mathrm{~mm}$ (kons. BB) bzW. 7,5 mm (konv. BB). In diesem Bereich konnten auf NRW $\mathrm{Nons}$ Anstiege des hEW gegenüber der Kontrolle festgestellt werden. Varianten, die besonders hohe plastische Verformungen $>6 \mathrm{~mm}$ hervorriefen, konnten jedoch nicht identifiziert werden.

Die Effekte auf $\mathrm{NRW}_{\text {konv }}$ sind deutlicher erkennbar, auch in Tiefen bis $39 \mathrm{~cm}$. In allen Jahren konnte ein steigender hEW mit steigender mechanischer Belastung festgestellt werden. Effekte in 2006 und 2007 sind in $5-14 \mathrm{~cm}$ insgesamt schwach ausgeprägt. Ursächlich hierfür kann die geringe Arbeitstiefe und ihr „Überschneiden“ mit der Spurtiefe sein. Für Radlastvarianten $>3,3 \mathrm{Mg}$ wurden Spurtiefen $>5 \mathrm{~cm}$ ermittelt, so dass ein Teil des obersten TASISSensors keinen Bodenwiderstand erfahren hat und auch die vertikale Spannung durch das konische Sensorende nicht aufgenommen wurde. GodWIN (2007) konnte zeigen, dass es für verschiedene Formen von Bodenbearbeitungsscharen kritische Arbeitstiefen gibt, bei deren Unterschreitung der Boden die Art seines Nachgebens („soil failure“) gegenüber dem Sensor verändert. So kann aus einer lateralen Verdrängung des Bodens (,lateral failure“) oberhalb der kritischen Arbeitstiefe eine vertikale Verdrängung („crescent failure“) resultieren, weil der vertikale Scherwiderstand des Bodens geringer ist als der der horizontale Spannungstensor. GoDWIN (2007) nennt für vertikale Schare im $90^{\circ}$ Winkel eine kritische Arbeitstiefen-Sensorbreiten-Relation (d/w-Relation) von 1. Die Betrachtungen von GoDWIN (2007) wurden nicht auf Sensorspitzen bezogen, ein hypothetischer Vergleich zeigt jedoch, dass TASIS bei einer Sensorbreite von $3 \mathrm{~cm}$ eine kritische Arbeitstiefe von $3 \mathrm{~cm}$ nicht unterschreiten dürfte. Unter planen Bodenbedingungen ist dieses auch bei einer flachen Sensorführung gegeben, in Spuren jedoch kann dieser Wert unterschritten werden. Ein verändertes Bruchverhalten des Bodens resultiert dann in einem niedrigeren Messwertniveau.

In 2008 konnten dennoch gute Messwerte aufgenommen werden, was zum einen daran lag, dass die Spurtiefen in diesem Jahr geringer waren als in 2006 und die durchschnittliche Messtiefe von TASIS in 2008 gegenüber 2007 leicht erhöht war. 
Neben den guten Ergebnissen aus $5-14 \mathrm{~cm}$ in 2008 konnten über alle drei Jahre auch abgestufte Datensätze in $18-27 \mathrm{~cm}$ ermittelt werden, die eine gute Übereinstimmung mit den plastischen Deformationsmessungen von ZINK (2009) zeigen. Trotz einer deutlich größeren Streuung der Messwerte ist dieses auch für $30-39 \mathrm{~cm}$ festzustellen. Eine Besonderheit zeigen die Daten aus $2008 \mathrm{im}$ Bereich $30-39 \mathrm{~cm}$, die trotz steigender technogener Belastung sinkende hEW aufweisen. Hierfür sind verschiedene Erklärungsansätze denkbar: zum einen kann die tiefere Sensorführung in 2008 dazu beigetragen haben, dass des EWMaximum (vgl. Abbildung 15) in diesem Jahr erfasst wurde, und dadurch eine veränderte Bodenreaktion auf mechanische Belastung erfasst wurde. Zum anderen gilt es zu bedenken, dass die Messungen in 2008 im Frühjahr stattfanden und somit ein saisonaler Effekt - trotz vergleichbarer Bedingungen - für eine unterschiedliche Messwertausprägung verantwortlich ist. Untersuchungen von HALVORSON ET AL. (2003), in denen der Einfluss des Winters auf Panzer-Fahrspuren untersucht wurde, kamen zu dem Ergebnis, dass regenerative saisonale Prozesse auf den Bereich $>5 \mathrm{~cm}$ Bodentiefe beschränkt bleiben. Indizien für eine gleichmäßige Änderung des Bodenverhaltens gegenüber Auflasten liefern die genannten Untersuchen nicht. Aufgrund der bereits angesprochenen methodischen Einschränkungen der Arbeit von HALVORSON ET AL. (2003) kann ein verändertes Bodenverhalten nach Belastungen durch landwirtschaftliche Fahrwerke jedoch ebenso wenig ausgeschlossen werden.

Einen dritten Erklärungsansatz liefern PETH ET AL. (2006) und FAZEKAS (2005), die aus Daten von Befahrungsversuchen mit Rübenrodern abgeleitet haben, dass hohe mechanische Lasteinträge ein Brechen der Plugsohle hervorrufen können. Nachfolgende Befahrungen können dann einen Spannungseintrag in tiefere Bodenschichten hervorrufen. Dieses wird von KOCH \& MÄRLÄNDER (2007) unter Berufung auf eigene Ergebnisse (bei denselben Befahrungsversuchen) und statistische Unstimmigkeiten bei PETH ET AL. (2006) angezweifelt. Und auch ZINK (2009) findet in seinen Messwerte keine Indizien auf ein Aufbrechen der Pflugsohle unter den gegebenen Bedingungen.

Die TASIS-Messwerte können die Theorie der genannten Autoren nicht verbindlich verifizieren. Sie wäre jedoch eine denkbare Begründung für das 
beobachtete Phänomen verringerter hEW bei höherer Belastung, da ein Durchdringen der Scharspitze durch einzelne Schollen vermutlich einen niedrigeren $\mathrm{hEW}$ erfordern würde als das Durchdringen eines fest gelagerten und in sich geschlossenen Horizontes.

ZINK (2009) stellte fest, dass die Bodenbearbeitung einen Einfluss auf die Spannungseinträge der Versuchsflächen hat, der in $20 \mathrm{~cm}$ und $60 \mathrm{~cm}$ Tiefe signifikant ist. Er gibt im Mittel der Mediane einen um 17\% geringeren aber nicht signifikanten Spannungseintrag bei konservierender Bodenbearbeitung an. Dieses deckt sich mit früheren Untersuchungen u. a. von HORN (1986), HORN (2004), FAZEKAS (2005). Die TASIS-Daten erlauben keine quantitative Bewertung des Faktors Bodenbearbeitung wie es die VP-Messungen tun, bei denen ein davor mit einem danach verglichen werden konnte. Aber die Betrachtung der Entwicklung der hEW auf den nordrhein-westfälischen Flächen zeigt, dass bei konservierender Bodenbearbeitung Zunahmen der hEW auf eine Messtiefe bis $27 \mathrm{~cm}$ beschränkt blieben, während sich auf NRW $_{\text {konv }}$ Einflüsse einer Befahrung auch noch in der Tiefe $30-39 \mathrm{~cm}$ ausprägten. Die Argumentation von ZiNK (2009), dass sich bei konservierender Bodenbearbeitung die Spannungen bereits in oberen Schichten stärker in der Horizontalen abstützen und dadurch weniger in die Tiefe fortsetzen, erscheint vor dem Hintergund der TASISErgebnisse nachvollziehbar. Eine quantitative Belastbarkeit können die TASISDaten hier aber nicht liefern.

Einen erkennbaren Unterschied des Bodenbearbeitungssystems auf die gewonnenen Daten konnten die Ergebnisse der Eingriffsintensität des Systems liefern (vgl. 4.5.5): Im Bereich des homogenisierten Bearbeitungshorizontes der konventionell bewirtschafteten Flächen zeigt sich, dass ein mehrfacher TASISEinsatz keinen störenden Effekt auf das junge Bodengefüge hat. Erst im Bereich unterhalb des Bearbeitungshorizontes wird dieser durch abnehmende hEW bei häufigerem Einsatz erkennbar. Bei flacherer Bodenbearbeitung auf den konservierend bewirtschafteten Flächen zeigen sich bereits im Bereich 18 $27 \mathrm{~cm}$ Unterschiede zwischen Erst- und Folgeeinsätzen von TASIS. Diese verstärken sich in $30-39 \mathrm{~cm}$, wobei in beiden Tiefenbereichen Unterschiede zwischen zweitem und dritten Einsatz nicht erkennbar sind. Dieses lässt schließen, dass eine Störung des Gefüges bei konservierender 
Bodenbearbeitung als nachhaltig $z u$ bewerten ist. Eine Regeneration des Gefüges innerhalb eines Jahres lässt sich anhand der Messdaten nicht vermuten. Es muss aber darauf hingewiesen werden, dass Messungenauigkeiten nicht ausgeschlossen werden können, da für die Befahrungen kein automatisches Lenksystem und auch kein hochgenaues RTK-Signal (Real Time Kinematik) genutzt wurde. Die Eingriffsintensität insbesondere beim Einsatz auf konservierend bewirtschafteten Flächen sollte bei weiterführenden Untersuchungen berücksichtigt werden.

Auch bei der Betrachtung des Einflusses einer Reifeninnendruckerhöhung auf den hEW ergeben sich Indizien, dass konservierend bewirtschaftete Flächen den höheren Spannungseintrag durch eine solche Maßnahme besser kompensieren können, als konventionell bewirtschaftete Flächen (vgl. Abbildung 30). Unterschiede konnten jedoch nicht statistisch abgesichert werden. Insgesamt bleibt der Einfluss der Anpassung des Reifeninnendruckes auf den hEW gering. Auch auf den sensitiven nordrhein-westfälischen Standorten konnte hier kein maßgeblicher Effekt festgestellt werden. Das System stößt an diesem Punkt unter Feldbedingungen an seine Grenzen bzgl. der Messwertauflösung. Der Einfluss des Faktors Radlast ist eindeutiger zu erkennen, da er unabhängig von Standort, Bodenbearbeitung und Messtiefe nachweisbar ist, allerdings vollziehen sich die Änderungen auch hier auf einem niedrigen Niveau, so dass nur über den Umfang der aufgenommenen Daten ein schwacher Einfluss unterstellt werden kann. TASIS erreicht bei der isolierten Betrachtung des Einflusses einzelner Parameter auch unter günstigen Voraussetzungen seine Grenzen. Die kombinierte Änderung der Parameter, also Erhöhung von Radlast und Reifeninnendruck, und ihr Einfluss auf die Bodenverdichtung bzw. den hEW können von TASIS aufgelöst werden. Voraussetzung hierfür sind allerdings skelettarme, homogene Böden und ein gewisser Stichprobenumfang, um die Ergebnisse abzusichern. Hier liefern die von ZINK (2009) genutzten Methoden zur Messung der Spannungseinträge ein differenzierteres Bild und vermögen den Einfluss des jeweiligen Faktors in unterschiedlichen Bodentiefen zu quantifizieren.

Die Variante Geo setzte sich erkennbar von den Varianten innerhalb des geordneten Versuchsdesigns ab (vgl. 4.5.4). Im Unterschied zur identischen 
Variante 7,5Mg@350kPa wurde bei ihr jedoch auf einer Fläche von 15 m * 15 m Fahrspur an Fahrspur gefahren. Hierbei wurden die Fläche durch den Zugschlepper ebenfalls nahezu flächendeckend mit allen Rädern überrollt, so dass ein wiederholter Lasteintrag in kurzer Zeitfolge auf die Fläche wirkte. Bei der Variante Geo konnten auch auf konservierend bewirtschafteten Flächen erkennbar erhöhte $\mathrm{hEW}$ in $30-39 \mathrm{~cm}$ gemessen werden, was auf eine mechanische Überschreitung der Tragfähigkeit schließen lässt. Untersuchungen von KOCH ET AL. (2007) zeigten, dass eine mehrfache Überrollung (hier: selbstfahrender Rübenroder, $12 \mathrm{Mg}$ Radlast) nahe Feldkapazität unabhängig von der Bodenbearbeitung Auswirkungen auf verschiedene Bodenkennwerte (vEW, Porenvolumen, Wasserleitfähigkeit) hat. Auch Untersuchungen von HORN ET AL. (2003), PETH \& HORN (2006) und PYTKA (2005) führten zu identischen Ergebnissen. Letztgenannter Autor konnte einen Anstieg von Spannungseinträgen und Bodendeformationen durch wiederholte Befahrung feststellen, hebt aber insbesondere die zweite Befahrung in ihrer Auswirkung auf die gemessenen Parameter hervor. Im Rahmen des Projektes von CAU und FH-SWF hat ZINK (2009) den Bereich GEO nicht gesondert beprobt, aber es wurden separate Messungen mit Mehrfachüberfahrungen durchgeführt, die zeigen, dass mit steigender Anzahl der Überfahrungen die Höhe der Spannungseinträge abnimmt und die plastische Gesamtverformung zunimmt. Die Ergebnisse der TASIS-Messungen zeigen, dass das System in der Lage ist, zwischen unterschiedlich stark belasteten Bereichen auch dann zu unterscheiden, wenn die Belastung auf eine Mehrfachbefahrung zurückgeht. Eine Differenzierung der Messwerte gegenüber einer einfachen aber intensiveren Belastung, bspw. durch eine höherlastige Variante kann jedoch nicht vorgenommen werden. TASIS kann aber zur Indentifizierung stark belasteter Bereiche innerhalb einer Fläche zur effizienten Planung bodenkundlicher Untersuchungen beitragen.

TASIS liefert im Vergleich zum VP differenziertere Datensätze. Sie lassen, zumindest auf den Lössstandorten, eine Abstufung der erfolgten mechanischen Belastung erkennen. Aussagekräftige Ergebnisse der VP-Messungen begrenzen sich hingegen auf punktuelle Ereignisse, die durch einen deutlichen Eingriff in die Bodenmechanik (z. B. durch wendende Bodenbearbeitung) oder 
die hydraulischen Kenngrößen (z. B. durch ein Starkregenereignis) geprägt wurden.

Der Versuch einer Bezugstellung der beiden Messgrößen in Korrelationsanalysen ergeben nur geringe Korrelationskoeffizienten von $r<0,7$, die sich - je nach Fläche und Messtiefenbereich - auch deutlich darunter bewegen können. In Untersuchungen anderer Autoren (HALL \& RAPER 2005, Siefken et AL. 2005, ChukWu \& Bowers 2005, Chung et AL. 2004) werden höhere Korrelationen genannt. Diese ergeben sich aber teilweise aus der NichtBerücksichtigung von Ausreißern (CHUNG ET AL. 2004).

In einer Gegenüberstellung von ADAMCHUK ET AL. (2006), in der die Systeme von CHUNG ET AL. (2003) und ADAMCHUK ET AL. (2008) verglichen werden, zeigen sich ähnlich niedrige Korrelationsgrade mit VP-Messungen $\left(r^{2}=0,32\right.$ bis 0,46$)$ wie in der vorliegenden Arbeit, jedoch gute Übereinstimmungen der Systemwerte untereinander $\left(r^{2}=0,57\right)$. Die Autoren führen die geringe Korrelation von vertikalen und horizontalen EW auf unterschiedliche Sensorgeometrien und Einsatzbedingungen zurück. Erstgenannter Grund kann auch im Falle der vorliegenden Arbeit das Maß an Übereinstimmung negativ beeinflusst haben. Bei identischem Sensoröffnungswinkel von $60^{\circ}$ waren Grundfläche und Oberfläche in ihrer Größe unterschiedlich. Darüber hinaus wurden die Systeme auch mit unterschiedlichen Penetrometergeschwindigkeiten geführt, die sich um den Faktor 13,5 unterschieden.

Die zweite Bezugstellung horizontaler und vertikaler EW stellte die Bestimmung des Ruhedruckkoeffizienten $\mathrm{K}_{0}$ dar (vgl. 4.6.2). TASIS liefert niedrigere Messwerte mit geringerer Streuung als das VP. Hieraus resultiert ein starker Einfluss der VP-Werte auf den Verlauf von $\mathrm{K}_{0}$ (vgl. Abbildung 38). Wie in 4.5.1 festgestellt, ist TASIS in der Lage, dichter lagernde Horizonte einer Fläche zu erfassen und dadurch eine Fläche zu charakterisieren. Die gewonnenen Daten korrelieren eng mit den VP-Daten, wenn sie auch nicht die zentimetergenaue Auflösung über die Messtiefe bieten können. Durch diese synchrone Entwicklung der Messdaten über die Messtiefenbereiche bedingt, erfährt $\mathrm{K}_{0}$ nur eine sehr geringe bis keine Änderung (vgl. Abbildung 37). Bereiche, die innerhalb der Einzeldatensätze auffällig sind, bspw. die Pflugsohlen der 
konventionell bewirtschafteten Flächen, werden durch $\mathrm{K}_{0}$ nicht abgebildet. Eine Verrechnung der durch TASIS und VP aufgenommenen Daten liefert hier keinen Erkenntnisgewinn, da ein konstanter Ruhedruckkoeffizient über die Tiefe nach BACHMANN ET AL. (2006) als charakteristisch für einen normalverdichteten Boden gesehen werden kann.

Keiner der ermittelten Werte lag oberhalb des von HARTGE \& HORN (1999) angegebenen Bereichs von 0,2 bis 0,7 . Dieses entspricht in etwa der Größenordnung der von SEMmEL (1993) und FAZEKAS (2005) genannten Werte. Beide Autoren konnten aus Daten eines Stress State Transducers, der multidirektionale Spannungen im Boden während einer mechanischen Belastung direkt erfasst, den Ruhedruckkoeffizienten direkt berechnen. Ihre Daten liegen in einem Bereich zwischen 0,05 und 0,56. FAZEKAS (2005) stellte klarere vertikale Spannungseinträge auf konservierend bewirtschafteten Flächen fest, während sie uneinheitliche Daten im Bereich der Pflugsohle im Sinne eines Auseindanderbrechens dieses Bereiches unter starker Last interpretierte.

Die Daten der vorliegenden Arbeit zeigen für unterschiedliche Bodenbearbeitungssysteme unterschiedliche Dynamiken von $\mathrm{K}_{0}$. Beobachtungen höherer Werte für $\mathrm{K}_{0}$ in befahrenen Bereichen beschränkten sich bei konservierender Bodenbearbeitung auf den oberflächennahen Bereich, während sie bei wendender Bodenbearbeitung im Bereich der Pflugsohle zu ermitteln waren. Dieses deutet darauf hin, dass nicht-wendend bearbeitete Flächen Auflasten bereits in oberflächennahen Schichten stärker in die Horizontale abstützen. Diese Aussage hat auch ZINK (2009) anhand seiner Daten treffen können. Bei Pflugeinsatz vermag der strukturschwache Oberboden dieses weniger effektiv. Die steigenden Werte von $\mathrm{K}_{0}$ deuten eine verstärkte horizontale Abstützung im Bereich der Pflugsohle und einen höheren Lasteintrag in diesem Messtiefenbereich an.

Beim Vergleich der eigenen Daten mit denen von FAZEKAS (2005) ist es wichtig hervorzuheben, dass es sich bei beiden Untersuchungen bei den verrechneten Spannungen um real aufgenommenen Messgrößen handelt, die mit nur mit geringer zeitlicher und räumlicher Variabilität bzw. zeitgleich aufgenommen 
wurden. In Untersuchungen anderer Autoren wurden horizontale Spannungen nicht direkt bestimmt, sondern der Ruhedruckkoeffizient wurde abgeleitet, bzw. substituiert. HARTGE \& BACHMANN (2004) nutzten in ihrem Modell hierfür vertikale Eindringwiderstände und errechneten den sog. Äquivalentruhedruck. Sie unterstellten hierbei, dass in $100 \mathrm{~cm}$ Bodentiefe durch die Abwesenheit externer mechanischer Belastungen ein hydrostatischer Zustand aller Hauptspannungen herrscht und keine Überverdichtung vorliegt. Des weiteren unterstellen die Autoren, dass an der Bodenoberfläche die Hauptspannungen gleich Null sind. Eine Gerade zwischen beiden Punkten (vEW in $100 \mathrm{~cm}$, vEW an der Oberfläche) beschriebe demnach den hydrostatischen Spannungszustand für jede dazwischen liegende Bodentiefe, mit deren Steigung dann ein Äquivalentruhedruck errechnet werden kann. Befindet sich ein real gemessener (vertikaler) EW oberhalb dieser Geraden, so kann eine Überkonsolidierung unterstellt werden. Ziel der Methode ist die Identifizierung verdichteter Feldbereiche für eine gezielte weitergehende bodenkundliche Untersuchung.

Eine Anwendung des Modells auf die Daten der vorliegenden Arbeit erfolgt unter der Einschränkung, dass Messdaten nur bis in eine Tiefe von $80 \mathrm{~cm}$ anstatt $100 \mathrm{~cm}$ vorliegen. Werden die untersten Messpunkte in Abbildung 15 durch eine Gerade gedanklich mit dem Koordinatenursprung verbunden, so befindet sich der überwiegende Anteil der Messwerte oberhalb dieser Geraden und eine Überkonsolidierung bereits vor Versuchsbeginn bis in tiefe Bodenschichten wäre zu unterstellen. Dieses scheint aufgrund der langen und intensiven ackerbaulichen Nutzung der einzelnen Flächen nicht ausgeschlossen, schließlich konnten BACHMANN ET AL. (2006) anhand von Untersuchungen an unterschiedlich intensiv genutzten Weideflächen diesen Effekt aufzeigen. Für eine Differenzierung unterschiedlich intensiv belasteter Flächen, wie im Rahmen der vorliegenden Arbeit durchgeführt, ist dieser methodische Ansatz aufgrund der benannten Limitierungen des VP ebenfalls nur stark eingeschränkt möglich. Beide Methoden eignen sich zur Flächencharakterisierung in Bezug auf ihre langjährige Nutzung mehr als zur Erfassung kurzfristiger Einflüsse. Der Ansatz von BACHMANN ET AL. (2006) erlaubt darüber hinaus eine bessere Bewertung der gewonnenen Flächendaten. 
Es muss festgehalten werden, dass das Konzept des Ruhedruckkoeffizienten $\mathrm{K}_{0}$ in seiner Aussagekraft von der Datenqualität aus vertikalen und horizontalen Spannungen abhängig ist. In der vorliegenden Arbeit konnte es nur einen geringen Erkenntnisgewinn liefern. Auch im Bereich seiner ursprünglichen Anwendung wird das Konzept diskutiert. So weist MICHALOWSKI (2005) auf die vereinfachten Grundannahmen der Herleitung sowie Diskrepanzen zwischen errechneten gegenüber gemessenen Ergebnissen aus experimentellen Untersuchungen hin.

Im Rahmen der Untersuchungen hat TASIS gezeigt, dass Änderung des EW, die aus einer mechanischen Belastung des Bodens resultieren, von dem System auch kleinräumig erfasst werden können. Um Aussagen über die Schadwirkung einer mechanischen Auflast treffen zu können, bedarf die Methode jedoch der Ergänzung bodenkundlicher Untersuchungen. Auf Basis der Ergebnisse von TASIS kann unter den gegebenen Bedingungen - es ist bekannt, wie stark die vorherige mechanische Belastung in bestimmten Bereichen war - die Aussage getroffen werden, dass in bestimmten Parzellen etwas passiert ist, jedoch nicht, was dort passiert ist und ob eine Bodenschadverdichtung daraus resultiert hat.

Die Daten von TASIS lassen jedoch folgende Schlussfolgerungen zu:

- Mechanische Belastungen bewirken Veränderungen (i. A. ein Ansteigen) des hEW.

- Die Veränderungen prägen sich auf Lössstandorten deutlicher aus als auf Geschiebemergel.

- Die Veränderungen nehmen mit steigender Belastungen i. d. R. zu.

- Flächen mit konventioneller BB zeigen ein höheres Maß an Veränderungen als konservierend bewirtschaftete Flächen, insbesondere in tieferen Messtiefen.

- Einflüsse der Fahrwerksparameter Radlast und Reifeninnendruck können mit der Methode im Ansatz nachgewiesen werden.

- Auf konservierend bewirtschafteten Flächen prägen sich Effekte vermehrt im Bereich des Oberbodens und weniger stark im Unterboden aus. 
- Steigende mechanische Auflasten können auch sinkende hEW bewirken. Eine abschließende Begründung hierfür konnte nicht gefunden werden.

- Mehrfache Überfahrungen führen zu einer Verstärkung von Effekten aus Einzelüberfahrten.

Die bodenkundlichen Untersuchungen der Fläche $\mathrm{NRW}_{\text {konv }}$ (vgl. 4.7) können einzelne dieser Schlussfolgerungen stützen. Im untersuchten Bereich von 36 $40 \mathrm{~cm}$ Tiefe konnten eine Abnahme der weiten Grobporen, geringere Wasserleitfähigkeiten und leicht erhöhte Lagerungsdichten in den befahrenen Parzellen (7,5Mg@350kPa, Geo) gegenüber der Kontrolle festgestellt werden (vgl. Tabelle 8). Ebenso unterscheiden sich diese Parzellen erkennbar gegenüber dem unbefahrenen Bereich bezüglich ihrer gesättigten Wasserleitfähigkeit und Luftkapazität (vgl. Abbildung 39). Die ohnehin nur mäßige Luftkapazität des Standortes wird durch die Befahrungen weiter reduziert und liegt in beiden Laststufen ausnahmslos unterhalb des von LEBERT ET AL. (2004) definierten Schadschwellenwertes von 5\% Vol. Unter der weiteren Berücksichtigung der Ergebnisse der Spatendiagnose (vgl. Tabelle 9) muss dieser Tiefenbereich nach dem Indikatormodell als schadverdichtet angesprochen werden.

Im Bereich des tieferen Unterbodens $(60$ - $64 \mathrm{~cm}$ ) liefern die eigenen Daten für Wasserleitfähigkeit und Luftkapazität keine Hinweise auf eine Schadverdichtung. Allerdings wurden die befahrenen Parzellen bei der Spatendiagnose in Bezug auf die Packungsdichte schlechter bewertet als die Kontrolle. Auch ZINK (2009) konnte auf den Lössstandorten in $60 \mathrm{~cm}$ Tiefe keine signifikante Abnahme der gesättigten Wasserleitfähigkeit durch einmalige Befahrung feststellen. Er hebt in seiner Arbeit jedoch hervor, dass andere Bodenkennwerte, insbesondere die Vorbelastung, auf eine Gefährdung auch dieses Tiefenbereiches durch hohe mechanische Auflasten schließen lassen.

Die vorliegende Arbeit liefert keine Daten, die verbindliche Forderungen in Form quantitativer Vorgaben, bspw. einer Begrenzung der maximalen Radlast, rechtfertigen. Die Ergebnisse der eigenen TASIS- und bodenkundlichen Messungen erlauben allerdings ebenso wenig einen leichtfertigen Umgang mit 
dem Thema Bodenverdichtung, sondern bekräftigen gemäß der Vorsorgepflicht $\S 7$ des BBodSchG Forderungen zur Nutzung des Bodens im Sinne der guten fachlichen Praxis (BBODSCHG 1998). Das BMVEL (2002) nennt als zentralen Ansatz zur Vorsorge gegen Bodenschadverdichtungen das Konzept des bodenschonenden Befahrens (DüRR ET AL. 1995, SOMmeR 1998, LeBERT ET AL. 2006). Dieses beinhaltet folgende Punkte:

- Verringerung des Kontaktflächendruckes durch größere Bereifung und die Absenkung des Reifeninnendruckes

- Anpassung von Arbeitsverfahren (Verwendung von Radialreifen, Kombination von Arbeitsgängen, Hundeganglenkung, GPSLenksysteme)

- Verbesserung der Belastungsfähigkeit der Böden durch angepasste und schonende Bodenbearbeitung

- Begrenzung der mechanischen Lasten

Die Ergebnisse der vorliegenden Arbeit können zur Stützung dieser vier Elemente herangezogen werden. Der Faktor Reifeninnendruck prägt sich in den Messdaten nur bedingt aus, hier erreicht Tasis seine Auflösungsgrenze. Unangepasste Arbeitsverfahren - im Falle von Tasis durch Mehrfachüberfahrungen im Bereich Geo repräsentiert - können aufgrund der Untersuchungen als kontraproduktiv für einen effektiven Bodenschutz bewertet werden, da die TASIS-Daten zeigen konnten, dass sich eine erhöhte Belastungshäufigkeit in erhöhten hEW ausdrückt. Diese bestätigen die bodenkundlichen Untersuchungen der Fläche NRW konv.

Auch eine intensive Bodenbearbeitung kann auf Basis der Tasis-Daten als unvorteilhaft in Bezug auf die Entwicklung der Eindringwiderstände angesehen werden, da sich Spannungseinträge durch Auflasten bis in tiefere Bodenschichten fortpflanzen.

Das MUNLV (2004) ergänzt zu den genannten Punkten noch die Aspekte der Beachtung des Wettergeschehens (d.h. in erster Linie der Bodenfeuchte) sowie der standörtlichen Gegebenheiten. Diese drücken sich, auch im Hinblick 
auf den letzten von SOMmeR (1998) angeführten Punkt, besonders deutlich in den Messergebnissen der Lössstandorte aus.

Die Vorgaben wurden nachfolgend zunehmend konkretisiert. Durch die BRANDHUBER ET AL. (2008) erfolgt dieses, indem sie für typische landwirtschaftliche Arbeitsgänge Vorschläge für eine praxisnahe Umsetzung der Vorgaben vorstellt. Neben den bereits genannten Maßnahmen führt das LUNG MV bei entsprechend großen Flächenstrukturen die Begrenzung der Schlaglängen als weitere Möglichkeit an, um doppelte Überfahrungen zu vermeiden. Diese Forderung findet sich auch in den „10 Geboten zur besten fachlichen Praxis im Bodenverdichtungsschutz" von SCHNEIDER \& SCHRÖDER (2005), die auch eine konzeptionelle Berücksichtigung der Erkenntnisse auf Seiten der Landtechnikindustrie in Form leichterer Erntemaschinen fordern. RAPER (2005) nennt die Einführung von Regelspursystemen („Controlled Traffic“) als weitere Möglichkeit einer Reduzierung der Bodengefährdung - trotz struktureller und verkehrsrechtlicher Einschränkungen wird die Einführung solcher Systeme mit der zunehmenden Verbreitung von GPS-Lenksystemen auch in Deutschland an Bedeutung gewinnen. Ein weiterer Aspekt, dessen Bedeutung von RAPER (2005) betont wird, ist die notwendige Achtsamkeit mit der Maßnahmen zur Tiefenlockerung erfolgen müssen. Auch Untersuchungen von SCHÄFER-LANDEFELD ET AL. (2004) zeigen, dass solche Maßnahmen kontraproduktiv für den Unterbodenschutz sein können. SPOOR (2006) nennt für den effektiven Einsatz die konstruktiven Kenngrößen. Die Tasis-Daten zeigen, dass für konservierend bewirtschaftete Flächen eine Gefügestörung resultieren kann, die sich im Laufe eines Jahres nicht regeneriert.

Als weitere umfangreiche Quelle bodenkundlicher und technischer Aspekte eines bodenschonenden Maschineneinsatzes im Sinne der guten landwirtschaftlichen Praxis sei an dieser Stelle ausdrücklich auf die VDIRichtlinie 6101 (VDI 2007) zum „Maschineneinsatz unter Berücksichtigung der Befahrbarkeit landwirtschaftlicher Böden“ hingewiesen. Sie berücksichtigt die meisten der angesprochenen Punkte und überführt sie in praxisrelevante Handlungsempfehlungen. 


\section{Fazit}

Eine technogene Verdichtung von landwirtschaftlichen Flächen, bspw. durch unsachgemäße Befahrung oder Bodenbewirtschaftung kann Bodenfunktionen nachhaltig beeinträchtigen. Eine genaue Kenntnis über die Wirkungen externer Spannungseinträge und anwendbare Methoden zur Identifizierung verdichteter Bodenareale sind daher erforderlich.

Im Rahmen der vorliegenden Arbeit wurde die Methode der Erfassung des Penetrometerwiderstandes in vertikaler und horizontaler Richtung genutzt, um Einflüsse mechanischer Auflasten auf den Eindringwiderstand zu quantifizieren, miteinander und mit Ergebnissen bodenkundlicher Untersuchungen zu vergleichen und Aussagen über die Eignung der angewandten Methode zur Erfassung von Bodenverdichtungen zu treffen.

Die Faktoren Standort (SH, NRW) mit den unterschiedlichen Bodenarten Geschiebemergel und Löss, die Bodenbewirtschaftung (konventionell, konservierend), sowie der Messtiefenbereich hatten eine starken Einfluss auf die Messwerte und überlagerten teilweise den Einfluss der untersuchten Fahrwerksparameter Radlast $(3,3 \mathrm{Mg}, 6,3 \mathrm{Mg}, 7,5 \mathrm{Mg})$ und Reifeninnendruck (niedrig, hoch) auf den Eindringwiderstand. Insbesondere das handgeführte Vertikalpenetrometer konnte hier nur eingeschränkte Aussagen im Hinblick auf differenzierte Ergebnisse liefern.

Das Sensorsystem TASIS hat sich konstruktiv bewährt und messtechnisch gute Ergebnisse geliefert. Einschränkungen müssen im Hinblick auf einen hohen Grobbodenanteil, starken Vegetationswuchs und die Differenzierung zwischen einfaktoriellen Änderungen von Fahrwerksparametern gemacht werden. Die gelieferten Daten liefern keine absoluten Werte, sondern bedürfen einer Interpretation, da höchste hEW nicht zwangsweise auf die höchste erfolgte mechanische Belastung schließen lassen. Allein auf Basis der TASIS-Daten lassen sich auch keine verbindlichen Aussagen über vorhandene Bodenschadverdichtungen treffen. Das System hat sich jedoch als geeignet erwiesen, auffällige Feldbereiche $z u$ identifizieren, auf denen bei gezielten bodenkundlichen Untersuchungen eine Schadverdichtung diagnostiziert werden 
konnte. TASIS kann damit wertvolle Entscheidungshilfen für den Ort von bodenkundlichen Untersuchungen liefern und helfen, Flächen gezielt auf mögliche Schadverdichtungen zu untersuchen. Das System kann so dazu beitragen, zügig eine größere Datenbasis zu erarbeiten, anhand derer dann ein Abgleich mit Daten aus Regressionsmodellen und deren Optimierung erfolgen kann.

Auch wenn keine quantitativen Forderungen aus den TASIS-Daten abgeleitet werden können, so bekräftigen sie die Forderungen nach einem vorsorgenden Bodenschutz zur nachhaltigen Nutzung unserer Böden und geben keinesfalls Anlass zur Entwarnung. Die vielfältigen Möglichkeiten Böden zu schützen, müssen im Rahmen der technisch und ökonomischen Möglichkeiten aber auch der ökologischen Erfordernisse genutzt werden.

Der konstruktive Ansatz von TASIs bietet aufgrund der großzügigen Geometrie der Sensoren und Schare darüber hinaus die Möglichkeit, eine Fusion von Sensorsystemen herbeizuführen. An erster Stelle wäre hier die Erfassung des Bodenwassergehaltes denkbar, aber auch Friktionsmessungen mit unterschiedlichen Scharformen, Materialien und Beschichtungen. Auch eine Anpassung von TASIS an technische Bodenbearbeitungssysteme für eine teilflächenspezifische, horizontgeführte Bodenlockerung könnte ein sinnvoller Transfer der gewonnenen Erkenntnisse sein. 


\section{Zusammenfassung}

Die nachhaltige Nutzung landwirtschaftlicher Böden und der Erhalt ihrer Produktions-, Lebensraum- und Regelungsfunktionen sind die Grundlage der Ernährungssicherung einer wachsenden Weltbevölkerung. Durch unsachgemäße Bodennutzung können Böden in ihrer Funktion gestört werden und Ertragsdepressionen resultieren. Starke mechanische Beanspruchung, bspw. langjährigen Pflugeinsatz oder die Befahrung mit schweren landwirtschaftlichen Maschinen unter ungünstigen Bedingungen können zu Bodenverdichtungen führen, die sich insbesondere in tieferen Bodenschichten nur langsam oder gar nicht regenerieren.

Im Rahmen eines interdisziplinären Forschungsprojektes sollten daher methodische Ansätze auf ihre Anwendbarkeit zur Erfassung von Bodenverdichtung untersucht werden. Die Erfassung des Eindringwiderstandes, also jener Kraft, die der Boden einer Sondenspitze entgegenbringt, ist ein anerkanntes Verfahren, um schnell Aussagen über die mechanischen Bodeneigenschaften in situ erheben zu können. Neben meist handgeführten Geräten (Vertikalpenetrometer, VP) zur Erfassung vertikaler Eindringwiderstände ( $\mathrm{VEW}$ ) sind in der Literatur traktorgezogene Horizontalpenetrometer beschrieben, die die großflächige Erfassung von Eindringwiderständen ermöglichen.

Im Rahmen des agrartechnischen Ansatzes des Forschungsprojektes wurde das Horizontalpenetrometersystem TASIS entwickelt, dass den horizontalen Eindringwiderstandes (hEW) in drei Tiefen erfasst. Auf konservierend (kons.) und konventionell (konv.) bewirtschafteten Flächen in Schleswig-Holstein (SH, Geschiebemergel) und Nordrhein-Westfalen (NRW, Löss) wurden unter definierten Bedingungen mechanische Belastungen in sechs Varianten bestehend aus drei Radlasten (3,3Mg, 6,3Mg, 7,5Mg) mit je zwei unterschiedlichen Reifeninnendrücken in einem geordneten Versuchsdesign angelegt und über einen Zeitraum von drei Jahren beprobt. Befahrungen fanden unter feuchten Bedingungen an zwei Terminen im Jahr (Frühjahr, Herbst) statt. Die Ergebnisse der TASIS-Messungen wurden mit 
Vertikalpenetrometermessungen und eigenen bodenkundlichen Untersuchungen sowie solchen des Projektpartners verglichen, um Möglichkeiten und Grenzen der Methodik zu bestimmen.

Vertikalpenetrometermessungen zeigten, dass auf beiden konventionell bewirtschafteten Flächen eine ausgeprägte Pflugsohle anzutreffen war. Ausgeprägte Effekte einer mechanischen Belastung konnten anhand von Vorher-Nachher-Vergleichen nur nach unmittelbar zuvor erfolgter wendender Bodenbearbeitung (BB) und nach starken Niederschlagsereignissen ermittelt werden und begrenzten sich auf die oberflächennahen Bodenschichten bis $27 \mathrm{~cm}$. Über den Versuchszeitraum konnten keine kumulativen Effekte durch mechanischen Lasteintrag identifiziert werden. Eine Differenzierung der eingebrachten mechanischen Auflasten anhand des vEW war auch auf den steinfreien Lössstandorten kaum möglich.

Der konstruktive Ansatz von TASIS ermöglichte, mit leichten Einschränkungen bei starkem Vegetationswuchs, auf allen Standorte eine sichere Messwerterfassung. Über die Datenaufnahme in drei Messtiefen $(5-14 \mathrm{~cm}$, $18-27 \mathrm{~cm}, 30-39 \mathrm{~cm}$ ) konnte die Bewirtschaftungspraxis der einzelnen Flächen erfolgreich abgebildet werden. Auf Geschiebemergel mit hohem Grobbodenanteil konnten nur teilweise stetig ansteigende hEW in Abhängigkeit der erfolgten mechanischen Belastung ermittelt werden.

Auf den verfahrenstechnisch günstigeren Lössstandorten konnten an allen Terminen abgestufte Effekte entsprechend dem Lasteintrag ermittelt werden, die sich bei konservierender BB auf die Messtiefenbereiche bis $27 \mathrm{~cm}$ beschränkten, während bei konventioneller $B B$ Einflüsse bis in $39 \mathrm{~cm}$ Tiefe nachzuweisen waren. Für 2008 wurden für diese gepflügte Flächen in 30 $39 \mathrm{~cm}$ sinkende hEW mit steigender Last festgestellt. Eine belastbare Erklärung konnte für dieses Phänomen nicht gefunden werden.

Mehrfachüberfahrungen führten zu einer weiteren Erhöhung des hEW und waren auch bei konservierender BB bis in Tiefen von $39 \mathrm{~cm}$ nachweisbar. Diese Ergebnisse korrespondieren mit den detaillierten bodenkundlichen Untersuchungen es Projektpartners. Einfaktorielle Änderungen von Radlast oder Reifeninnendruck innerhalb der Versuchsandordnung zeigten die 
Systemgrenzen von TASIS auf und konnten nur in Ansätzen und auf den Lössstandorten dargestellt werden. Der wiederholte Einsatz von TASIS an derselben Stelle in darauffolgenden Jahren zeigte, dass das System einen Eingriff in das Bodengefüge darstellt, der sich auf konservierend bewirtschafteten Flächen stärker ausprägte.

Korrelationen von vEW und hEW waren gering $(r=0,60)$ und unterschieden sich stark in Abhängigkeit von Fläche und Messtiefe. Der ermittelte Ruhedruckkoeffizient $\left[K_{0}\right]$ bewegte sich bei allen Messungen im Bereich normalverdichteter Böden und konnte genutzt werden, um die Ergebnisse der bodenkundlichen Untersuchungen zu stützen, nach denen eine verstärkte Lastabstützung bei konservierenden $\mathrm{BB}$ in oberen Bodentiefen und bei konventioneller BB in tieferliegenden Schichten stattfindet.

Stark belastete Feldbereiche, die innerhalb der TASIS-Daten eine exponierte Stellung einnahmen, konnten in einer nachfolgenden bodenkunflichen Untersuchung als schadverdichtet nach dem Indikatorenmodell von LEBERT ET AL. (2004) angesprochen werden. Ein Einsatz von TASIS zur Identifizierung gefährdeter Bereich kann eine gezielte bodenkundliche Flächenbeprobung ermöglichen.

Anhand der TASIs-Ergebnisse kann ein großer Teil der ausformulierten Anforderungen an eine gute fachliche Praxis zur bodenschonenden Befahrung nachvollzogen werden. Der Schutz der Böden erfordert ein „Drehen an vielen Stellschrauben“ und ein Bewußtsein für die Bedeutung der Erhaltung dieses Gutes. 


\section{Abstract}

Sustainable use of arable soil is essential for world food security. Soil degradation by over-compaction is counteracting this goal. Long-term conventional tillage (plough) and heavy agricultural machinery use under wet soil conditions put arable soils at harm.

An interdisciplinary research project was launched in order to assess the effect of mechanical stress induction on arable soils and to evaluate different methods of identifying the impact on soils.

Vertically operated cone penetrometers (VP) have been in use for many years to allow an in situ assessment of soil conditions. Also horizontally operated penetrometers have been described by different authors. In line with project goals the multi-sensor penetrometer-system TASIS has been developed, enabling data acquisition of penetration force in three depths simultaneously. $A$ large scale field experiment design was implemented taking into account two different soils (loess, soils derived from glacial till deposits), both concervation (mulch) and conventional (plough) tillage, three wheel loads (3.3Mg, 6.3Mg, $7.5 \mathrm{Mg}$ ) with two different tire inflation pressures (high, low) applied at each load. Wheeling was conducted under wet soil conditions twice a year.

VP results identified the long term tillage effect on soil layer characteristics. Distinct effects of mechanical stress induction on penetration resistance could be shown either shortly after ploughing or with a severe change of soil water conditions. Sophisticated results in terms of wheel load or tire inflation pressure could not be identified. Accumulating effects of soil compaction over project run time was not observed with VP measurements.

TASIS successfully identified layers of different degrees of compactness. A considerable stone fraction in till deposits limited the identification of compacting effect of distinct mechanical stress induction. Loess soils proved to be more susceptible to TASIS measurements. At all dates gradual effects according to the mechanical stresses applied could be identified. With conservation tillage those effects were limited to soil depths of $28 \mathrm{~cm}$ or less, while ploughed fields were impacted down to $39 \mathrm{~cm}$. Repeated wheeling resulted in increasing 
horizontal penetration resistance unto depth of $39 \mathrm{~cm}$ also on conservation tillage plots. Repeated use of TASIS in the same slot revealed a soil disturbing effect of the system, which has to be considered specially on conservation tillage field.

All results match to a good part with results of the soil physics measurements. Effects of either wheel load alteration or tire inflation pressure alteration could not be distinctly identified by TASIS, but with soil physical analysis. Small alteration of either load or tire inflation pressure show the technical limitations of the system.

Calculation of the coefficient of earth pressure at rest $\left[\mathrm{K}_{0}\right]$ suggest that with conservation tillage vertical stresses are transferred to the horizontal plane in soil layers near the surface, whilst on ploughed plots stresses propagate to deeper soil layers.

TASIS proved to be more sensitive to mechanical stress induction than established VP-methodology. Peculiar field plots identified by TASIS data showed to be over-compacted when analyzed by soil physical methods. Hereby foundations are laid to use work and cost-intensive soil physical analysis more selectively by pre-assesing field using TASIS. 


\section{Literaturverzeichnis}

Adamchuk, V.I., Skotnikov, A.V., Speichinger, J.D. \& Kocher, M.F. (2004a):

Development of an instrumented deep-tillage implement for sensing of soil mechanical resistance. Trans. ASAE, 47 (6), 1913-1919.

Adamchuk, V.I., Hummel, J.W., Morgan, M.T. \& Upadhyaya, S.K. (2004b): Onthe-go soil sensors for precision agriculture. Comput. Electron. Agric., 44, 71-91.

Adamchuk, V.I., Sudduth, K.A., Ingram, T.J. \& Chung, S.-O. (2006): Comparison of two alternative methods to map soil mechanical resistance on-the-go. In: Proc. 2006 ASABE Annual International Meeting, Portland, Oregon, USA, Paper No. 061057.

Adamchuk, V.I., Ingram, T.J., Sudduth, K.A. \& Chung, S.O. (2008): On-the-go mapping of soil mechanical resistance using a linear depth effect model. Trans. ASABE, 51 (6), 1885-1894.

AG Boden (2005): Bodenkundliche Kartieranleitung (KA5) (5. Auflage).

Schweitzerbart'sche Verlagsbuchhandlung, Stuttgart 2005.

Alihamsyah, T., Humphries, E.G. \& Bowers, C.G.Jr. (1990): A technique for horizontal measurement of soil mechanical impedance. Trans. ASAE, 33 (1), 73-77.

American Society of Agricultural Engineers (ASAE) (Hrsg.) (1999): Procedures for using and reporting data obtained with the soil cone penetrometer (ASAE EP542: 1999). St. Joseph, Michigan, USA.

ASTM International (Hrsg.) (2004): Standard D3441 - Standard method of deep quasi-static cone and friction-cone penetration tests of soil. West Conshohocken, Pennsylvania, USA.

Bachmann, J., Contreras, K., Hartge, K.H. \& MacDonald, R. (2006):

Comparison of soil strength data obtained in situ with penetrometer and with vane shear test. Soil Tillage Res., 87, 112-118. 
BBodSchG: Gesetz zum Schutz vor schädlichen Bodenveränderungen und zur Sanierung von Altlasten vom 17.03.1998. BGB I, S. 502.

Bayerische Landesanstalt für Landwirtschaft (LFL)\& Sächsische Landesanstalt für Landwirtschaft (Hrsg.) (2005): LfL-Information - Bodenstruktur erkennen und beurteilen - Anleitung zur Bodenuntersuchung mit dem Spaten (4. geänderte Auflage). Freising-Weihenstephan 2005.

Becher, H.H. (2005): Auswirkung von Reifenbreite, Spurbreite und Radstand auf die Druckverteilung unter einem landwirtschaftlichen Fahrzeug. Mitt. DBG, 107, 23-24.

Bölenius, E., Rogstrand, G., Arvidsson, J., Stenberg, B. \& Thylen, L. (2006): Onthe-go measurements of soil penetration resistance on a Swedish Eutric Cambisol. In Nordic Association of Agricultural Scientists (Hrsg.): NJF Report, NJFSeminar 378 - Tillage systems for the benefit of agriculture and the environment, Vol. 2, Nr. 4, S. 106-109.

Boussinesq, J. (1885): Application des potentials á l'etude de l'equilibre et du movement des solides elastique. Gauthier-Villars, Paris 1885.

Brandhuber, R., Demmel, M., Koch, H.-J. \& Brunotte, J. (2008):

Bodenschonender Einsatz von Landmaschinen - Empfehlungen für die Praxis. In DLG e.V. \& Bayerische Anstalt für Landwirtschaft (Hrsg.): DLGMerkblatt 344 (1. Auflage). Frankfurt am Main 2008.

Brosius, F. (2004): SPSS 12 (1. Auflage). mitp-Verlag, Bonn 2004.

Brunotte, J., Sommer, C. \& Lebert, M. (2001): Ein praxisorientiertes Konzept mit Lösungsansätzen und Ergebnissen zur Vorsorge gegen Bodenschadverdichtungen. In: 14. Wissenschaftliche Fachtagung der Landwirtschaftlichen Fakultät der Universität Bonn. Teil 2, 136-149.

Brunotte, J., Lorenz, M., Sommer, C., Harrach, T. \& Schäfer, W. (2008): Verbreitung von Bodenschadverdichtungen in Südniedersachsen. In Bundesministerium für Ernährung, Landwirtschaft und Verbraucherschutz (Hrsg.): Berichte über Landwirtschaft, Band 86, Heft 2. Kohlhammer Verlag, Stuttgart 2008, S. 262-284. 
Bundesanstalt für Geowissenschaften und Rohstoffe (BGR) (2008): Karte der Bodengroßlandschaften der Bundesrepublik Deutschland 1:5.000.000. http://www.bgr.bund.de/cln_092/nn_325378/DE/Themen/Boden/Produkte /Karten/BGL_5000.html (aufgerufen am 28.11.2008).

Bundesministerium für Umwelt, Naturschutz und Reaktorsicherheit (BMU) (Hrsg.) (2009): Zweiter Bodenschutzbericht der Bundesregierung. Drucksache 16/12658 vom 15.04.2009.

Bundesministerium für Verbraucherschutz, Ernährung und Landwirtschaft (BMVEL) (Hrsg.) (2002): Gute fachliche Praxis zur Vorsorge gegen Bodenschadverdichtungen und Bodenerosion. Bonn.

Chukwu, E. \& Bowers, C.G.Jr. (2005): Instantaneous multiple-depth soil mechanical impedance sensing from a moving vehicle. Trans. ASAE, 48 (3), 885-894.

Chung, S.-O., Sudduth, K.A \& Hummel, J.W. (2003): On-the-go soil strength profile sensor using a load cell array. In: Proc. 2003 ASAE Annual International Meeting, Las Vegas, Nevada, USA, Paper No. 031701.

Chung, S.-O., Sudduth, K.A., Plouffe, C. \& Kitchen, N.R. (2004): Evaluation of an on-the-go soil strength profile sensor using soil bin and field data. In: Proc. 2004 ASAE/CSAE Annual International Meeting, Ottawa, Ontario, Canada, Paper No. 041039.

Cramer, B. (2006): Überprüfung von Bewertungsmodellen zur Identifikation und Prognose von Schadverdichtungen auf Ackerböden in NordrheinWestfalen. Dissertation Universität Bonn.

Danfors, B. (1994): Changes in subsoil porosity caused by heavy vehicles. Soil Tillage Res., 29, 135-144.

Denker, S., Volk, L. \& Lücke, W. (2007): Systematic approaches of soil mechanical stress induction in arable lands - Impact assessment and implications for energy efficiency enhancement in agricultural engeneering. In: Proc. 65th International Conference on Agricultural Engeneering LAND.TECHNIK - AgEng 2007, Hannover, Deutschland, 441-445. 
Desbiolles, J.M.A., Godwin, R.J., Kilgour, J. \& Blackmore, B.S. (1999):

Prediction of tillage implement draught using cone penetrometer data. J. Agric. Eng. Res., 73, 65-76.

Deutsches Institut für Normung e.V. (DIN) (Hrsg.) (1998a): DIN 19682-10:1998.

Bodenuntersuchungsverfahren im Landwirtschaftlichen Wasserbau -

Felduntersuchungen - Teil 10: Beschreibung und Beurteilung des Bodengefüges (ersetzt durch DIN 19682-10:2007). Beuth Verlag, Berlin 1998.

Deutsches Institut für Normung e.V. (DIN) (Hrsg.) (1998b): DIN 19683-9:1998.

Bodenuntersuchungsverfahren im Landwirtschaftlichen Wasserbau Physikalische Laboruntersuchungen - Teil 9: Bestimmung der

Wasserdurchlässigkeit in wassergesättigten Stechzylinderproben. Beuth Verlag, Berlin 1998.

Deutsches Institut für Normung e.V. (DIN) (Hrsg.) (2002): DIN ISO 11508.

Bodenbeschaffenheit - Bestimmung der Kornrohdichte (ISO 11508: 1998). Beuth Verlag, Berlin 2002.

Dexter, A.R., Czyz, E.A. \& Gate, O.P. (2007): A method for prediction of soil penetration resistance. Soil Tillage Res., 93, 412-419.

Diserens, E. \& Bucher, F. (1997): Bodenverdichtung aus Sicht der Bodenmechanik. Landtechnik, 4 (2), 83-86.

Dürr, H.-J., Petelkau, H. \& Sommer, C. (1995): Literaturstudie "Bodenverdichtung". In Umweltbundesamt (Hrsg.): Texte, Heft 55, Berlin 1995.

Ehlers, W., Werner, D. \& Mähner, T. (2000): Wirkung mechanischer Belastung auf Gefüge und Ertragsleistung einer Löss-Parabraunerde mit zwei Bearbeitungssystemen. J. Plant Nutr. Soil Sci., 163, 321-333.

Eijkelkamp Agrisearch Equipment (2000): Gebrauchsanweisung PenetrologgerSet 06.15. Giesbeek, Niederlande. 
Fazekas, O. (2005): Bedeutung von Bodenstruktur und Wasserspannung als stabilisierende Kenngrößen gegen intensive mechanische Belastungen in einer Parabraunerde aus Löss unter Pflug- und Mulchsaat. Dissertation Universität Kiel.

Fazekas, O., Horn, R. \& Hamann, S. (2005): Bodenstabilitätsänderung unter schweren landwirtschaftlichen Maschinen bei unterschiedlichen Bodenbearbeitungen. Mitt. DBG, 107, 39-40.

Flowers, M. \& Lal, R. (1998): Axle load and tillage effect on soil physical properties and soybean grain yield on a mollic ochraqualf in Northwest Ohio. Soil Tillage Res., 48, 21-35.

Food and Agriculture Organization of the United Nations (FAO) (2010): "Climate-smart" agriculture - Policies, practices and financing for food security, adaptation and mitigation. In: Proc. Hague Conference on Agriculture, Food Security and Climate Change, Den Haag, Niederlande.

Fröhlich, O.K. (1934): Druckverteilung im Baugrunde mit besonderer Berücksichtigung der plastischen Erscheinungen. Julius Springer Verlag, Wien 1934.

Garciano, L.O., Upadhyaya, S.K., Jones, R.A. \& Jersey, S.R. (2007): Determination of the soil pressure distribution around a cone penetrometer. J. Terramech., 44, 265-273.

Gill, W.R. \& Vanden Berg, G.E. (1967): Soil dynamics in tillage and traction. In United States Department of Agriculture (Hrsg.): Agriculture Handbook, No. 316. Auburn, Alabama, USA, S. 460-487.

Godwin, R.J. (2007): A review of the effect of implement geometry on soil failure and implement forces. Soil Tillage Res., 97, 331-340.

Gorucu, S., Khalilian, A., Han, Y.J., Dodd, R.B. \& Smith, B.R. (2006): An algorithm to determine the optimum tillage depth from soil penetrometer data in coastal plain soils. Appl. Eng. Agric., 22 (5), 625-631.

Gysi, M., Ott, A. \& Flühler, H. (1999): Influence of single passes with high wheel load on a structured, unploughed sandy loam soil. Soil Tillage Res., 52, 141-151. 
Gysi, M., Maeder, V. \& Weisskopf, P. (2001): Pressure distribution underneath tires of agricultural vehicles. Trans. ASAE, 44 (6), 1385-1389.

Hakansson, I. \& Reeder, R.C. (1994): Subsoil compaction by vehicles with high axle load - extent, persistence and crop response. Soil Tillage Res., 29, 277-304.

Hall, H.E. \& Raper, R.L. (2005): Development and concept evaluation of an onthe-go soil strength measurement system. Trans. ASAE, 48 (2), 469-477.

Halvorson, J.J., Gatto, L.W. \& McCool, D.K. (2003): Overwinter changes to near-surface bulk density, penetration resistance and infiltration rates in compacted soil. J. Terramech., 40, 1-24.

Hammel, K. (1994): Soil stress distribution under lugged tires. Soil Tillage Res., 32, 163-181.

Hamza, M.A. \& Anderson, W.K. (2005): Soil compaction in cropping systems: A review of the nature, causes and possible solutions. Soil Tillage Res., 82, $121-145$.

Hartge, K.H. (1986): A concept of compaction. Z. Pflanzenernähr. Bodenk., 4, 361-370.

Hartge, K.H. \& Horn, R. (1992): Die physikalische Untersuchung von Böden (3. Auflage). Ferdinand Enke Verlag, Stuttgart 1992.

Hartge, K.H. \& Horn, R. (1999): Einführung in die Bodenphysik. Ferdinand Enke Verlag, Stuttgart 1999.

Hartge, K.H. \& Bachmann, J. (2004): Ermittlung des Spannungszustandes von Böden aus Werten des Eindringwiderstandes von Sonden. J. Plant Nutr. Soil Sci., 167, 303-308.

Hatch, R., Sharpe, T. \& Galyean, P. (2002): A global high accuracy differential GPS system. In: Proc. 2003 National Technical Meeting of The Institute of Navigation, Anaheim, Canada, 562-573.

Henderson, C., Levett, A. \& Lisle, D. (1988): The effects of soil water content and bulk density on the compactibility and soil penetration resistance of some Western Australian sandy soils. Aust. J. Soil Res., 26, 391-400. 
Hemmat, A. \& Adamchuk, V.I. (2008): Sensor systems for measuring soil compaction: Review and analysis. Comput. Electron. Agric., 63, 89-103.

Heuer, H., Tomanová, O., Koch, H.-J. \& Märländer, B. (2008): Subsoil properties and cereal growth as affected by a single pass of heavy machinery and two tillage systems on a Luvisol. J. Plant Nutr. Soil Sci., 171, 580-590.

Hoffmann, J. (2004): Handbuch der Messtechnik (2. aktualisierte und erweiterte Auflage). Carl Hanser Verlag, München Wien 2004.

Horn, R. (1986): Auswirkung unterschiedlicher Bodenbearbeitung auf die mechanische Belastbarkeit von Ackerböden. Z. Pflanzenernähr. Bodenk., 149, 9-18.

Horn, R. (2004): Time dependence of soil mechanical properties and pore functions for arable soils. Soil Sci. Soc. Am. J., 68, 1131-1137.

Horn, R., Baumgartl, T., Kühner, S., Lebert, M. \& Kayser, R. (1991): Zur Bedeutung des Aggregierungsgrades für die Spannungsverteilung in strukturierten Böden. Z. Pflanzenernähr. Bodenk., 154, 21-26.

Horn, R. \& Fleige, H. (2003): A method for assessing the impact of load on mechanical ability and on physical properties of soils. Soil Tillage Res., 73, 89-99.

Horn, R., Way, T. \& Rostek, J. (2003): Effect of repeated tractor wheeling on stress/strain properties and consequences on physical properties in structured arable soils. Soil Tillage Res., 73, 101-106.

Johnson, C.E. \& Burt, E.C. (1990): A method of predicting soil stress state under tires. American Society of Agricultural Engeneers, 33 (3), 713-717.

Keller, T., Trautner, A. \& Arvidsson, J. (2002): Stress distribution and soil displacement under a rubber-tracked and a wheeled tractor during ploughing, both on-land and within furrows. Soil Tillage Res., 68, 39-47.

Keller, T. \& Arvidsson, J. (2004): Technical solutions to reduce the risk of subsoil compaction: effects of dual wheels, tandem wheels and tyre inflation pressure on stress propagation in soil. Soil Tillage Res., 79, 191-205. 
Koch, H.-J., Heuer, H. \& Tomanová, O. (2007): Soil properties and sugar beet growth as affected by repeated passes of heavy machinery and soil tillage. In: Proc. 65th International Conference on Agricultural Engeneering LAND.TECHNIK - AgEng 2007, Hannover, Deutschland, 447-453.

Koch, H.-J. \& Märländer, B. (2007): Letter to the editor - Comment on "Heavy soil loading and its consequences for soil structure, strength and deformation of arable soils" by S. Peth, R. Horn, O. Fazekas and B. G. Richards. J. Plant Nutr. Soil Sci., 170, 693-695.

Koch, H.-J., Heuer, H., Tomanová, O. \& Märländer, B. (2008): Cumulative effect of annually repeated passes of heavy agricultural machinery on soil structural properties and sugar beet yield under two tillage systems. Soil Tillage Res., 101, 69-77.

Landesamt für Umwelt, Naturschutz und Geologie Mecklenburg-Vorpommern (LUNG MV) (Hrsg.) (o. J.): Beiträge zum Bodenschutz in MecklenburgVorpommern - Bodenverdichtung.

Lapen, D.R., Topp, G.C., Edwards, M.E., Gregorich, E.G. \& Curnoe, W.E. (2004): Combination cone penetration resistance/water content instrumentation to evaluate cone penetration-water content relationships in tillage research. Soil Tillage Res., 79, 51-62.

Lebert, M., Brunotte, J. \& Sommer, C. (2004): Ableitung von Kriterien zur Charakterisierung einer schädlichen Bodenveränderung, entstanden durch nutzungsbedingte Verdichtung von Böden / Regelung zur Gefahrenabwehr. In Umweltbundesamt (Hrsg.): Texte, Heft 46, Berlin 2004.

Lebert, M., Brunotte, J., Sommer, C. \& Böken, H. (2006): Bodengefüge gegen Verdichtungen schützen - Lösungsansätze für den Schutz landwirtschaftlich genutzter Böden. J. Plant Nutr. Soil Sci., 169, 633-641. 
Lindner, H. (1964): Bodenphysikalische Untersuchungen über den Einfluss verschiedener Pflugkörper bei unterschiedlichen Arbeitsgeschwindigkeiten auf einige Eigenschaften des Bodens. Habilitationsschrift 118, Humboldt-Universität Berlin.

Lunne, T., Robertson, P.K. \& Powell, J.J.M. (1997): Cone penetration testing in geotechnical practice (1. Auflage). Blackie Academic and Professional, London 1997.

Lüth, H.-G. (1993): Entwicklung eines Längs-Penetrographen als Meßverfahren zur Bodenverdichtung. Dissertation Universität Kiel.

Mähner, K.-T. (1999): Wachstum und Ertrag von Getreide nach mechanischer Bodenbelastung in unterschiedlichen Bearbeitungssystemen. Dissertation Universität Göttingen.

Meynen, E., Schmithüsen, J., Gellert, J., Neef, E., Müller-Miny, H. \& Schultze, J.H. (Hrsg.) (1962): Handbuch der naturräumlichen Gliederung Deutschlands. Selbstverlag der Bundesanstalt für Landeskunde und Raumordnung, Bad Godesberg 1962.

Michalowski, R.L. (2005): Coefficient of earth pressure at rest. J. Geotech. Geoenviron. Eng., 131, 1429-1433.

Michelin Reifenwerke KGaA (2006): Betriebsanleitung Landwirtschaftsreifen Ausgabe 6. Karlsruhe.

Ministerium für Umwelt und Naturschutz, Landwirtschaft und Verbraucherschutz des Landes Nordrhein-Westfalen (MUNLV) (Hrsg.) (2004): Bodenschutz in der Landwirtschaft, Düsseldorf 2004.

National Aeronautics and Space Administration (NASA) (Hrsg.) (2001): NASA Facts: Did U.S. astronauts really land on the moon? Washington, USA. Nederlands Normalisatie-Instituut (NEN) (Hrsg.) (1996): Geotechniek Bepaling van de conusweerstand en de plaatselijke wrijvingsweerstand van grond - Elektrische sondeermethode (NEN 5140:1996 nl). 
Paul, R. (2004): Verfahren zur Ermittlung der Schadverdichtungsrisiken auf ackerbaulich genutzten Böden. In Thüringer Ministerium für Landwirtschaft, Naturschutz und Umwelt (Hrsg.): Zwischenbericht 46.02. Jena 2004

Petelkau, H. (1989): Arbeiten zur Mechanisierung der Pflanzen- und Tierproduktion. Schlieben, 6 (43), 93-114.

Petelkau, H., Seidel, K. \& Frielinghaus, M. (2000): Ermittlung des Verdichtungswiderstandes von Böden des Landes Brandenburg und Bewertung von Landmaschinen und landwirtschaftlichen Anbauverfahren hinsichtlich der Beeinträchtigung von Bodenfunktionen durch die Verursachung von schwer regenerierbaren Schadverdichtungen. ZALF Müncheberg e.V. im Auftrag des Ministeriums für Umwelt, Naturschutz und Raumordnung des Landes Brandenburg.

Peth, S. \& Horn, R. (2004): Zur Abschätzung von Bodenspannungen unter landwirtschaftlichen Nutzfahrzeugen. Landtechnik, 5, 268-269.

Peth, S. \& Horn, R. (2006): The mechanical behavior of structured and homogenized soil under repeated loading. J. Plant Nutr. Soil Sci., 169, 401-410.

Peth, S., Horn, R., Fazekas, O. \& Richards, B.G. (2006): Heavy soil loading an its consequences for soil structure, strength and deformation of arable soils. J. Plant Nutr. Soil Sci., 169, 775-783.

Pytka, J. (2005): Effects of repeated rolling of agricultural tractors on soil stress and deformation state in sand and loess. Soil Tillage Res., 82, 77-88.

Pytka, J., Dabrowski, J., Zajac, M. \& Tarowski, P. (2006): Effects of reduced inflation pressure and vehicle loading on off-road traction and soil stress and deformation state. J. Terramech., 43, 469-485.

Raper, R.L. (2005): Agricultural traffic impacts on soil. J. Terramech., 42, 259280.

Ruhm, E. (1983): Schlechte Voraussetzungen für eine gute Ernte. Hannover. Land- und forstw. Zeitung, 136 (4), 3-4. 
Schäfer-Landefeld, L., Brandhuber, R., Fenner, S., Koch, H.-J. \& Stockfisch, N. (2004): Effects of agricultural machinery with high axle load on soil properties of normally managed fields. Soil Tillage Res., 75, 75-86.

Scheffer, F. \& Schachtschabel, P. (2002): Lehrbuch der Bodenkunde (15. Auflage). Spektrum Akademischer Verlag, Heidelberg 2002.

Scherzer, J., Schaaf, W. \& Hüttl, R.F. (1996): Eignung von FDR- und TDRSonden zur Erfassung der Bodenfeuchte in Kippsubstraten mit erhöhter elektrischer Leitfähigkeit. Mitt. DBG, 80, 279-282.

Schneider, R. \& Schröder, D. (2005): Degressive Kontaktflächen- und Reifeninnendrücke bei steigenden Radlasten als präventive Indikatoren für tolerierbare mechanische Bodenbelastungen. In Bundesministerium für Ernährung, Landwirtschaft und Verbraucherschutz (Hrsg.): Berichte über Landwirtschaft, Band 83, Heft 2. Kohlhammer Verlag, Stuttgart 2005, S. 195-224.

Schulze Lammers, P., Sun, Y. \& Ma, D. (2007): Transient detection of soil water content and mechanical resistance by a horizontal penetrometer combined with an impedance sensor. In: Proc. 2007 ASABE Annual International Meeting, Minneapolis, Minnesota, USA, Paper No. 071007.

Schwark, A. (2005): Bewirtschaftung und Status von Ackerböden in SchleswigHolstein. Dissertation Universität Kiel.

Semmel, H. (1993): Auswirkungen kontollierter Bodenbelastungen auf das Druckfortpflanzungsverhalten und physikalisch-mechanische Kenngrößen von Ackerböden. Dissertation Universität Kiel.

Sharifi, A., Godwin, R.J., O’Dogherty, M.J. \& Dresser, M.L. (2007): Evaluating the performance of a soil compaction sensor. Soil Use and Management, 23, 171-177.

Sharpe, T., Hatch, R. \& Nelson, F. (2000): John Deere's StarFire system: WADGPS for precision agriculture. In: Proc. 13th International Technical Meeting of the Satellite Division of the Institute of Navigation, Salt Lake City, Utah, USA, 2269-2277. 
Siefken, R.J., Adamchuk, V.I., Eisenhauer, D.E \& Bashford, L.L. (2005): Mapping soil mechanical resistance with a multiple blade system. Appl. Eng. Agric., 21 (1), 15-23.

Soane, B.D. \& van Ouwerkerk, C. (1994): Soil compaction problems in world agriculture. In Soane, B.D. \& van Ouwerkerk, C. (Hrsg.): Developments in agricultural engeneering 11 - Soil compaction in crop production. Elsevier, Amsterdam 1994, S. 1-21.

SöHNE, W. (1951): Das mechanische Verhalten des Ackerbodens bei Belastungen, unter rollenden Rädern sowie bei der Bodenbearbeitung. Grdlgn. d. Landtechn., 1, 87-94.

Söhne, W. (1953): Druckverteilung im Boden und Bodenverformung unter Schlepperreifen. Grdlgn. d. Landtechn., 5, 49-63.

Söhne, W. (1956): Einige Grundlagen für eine Landtechnische Bodenmechanik. Grdlgn. d. Landtechn., 7, 11-27.

Sommer, C. (1998): Ein Konzept zur Vorbeugung von Bodenschadverdichtungen in der pflanzlichen Produktion. Bodenschutz, 1, 12-16.

Spoor, G. (2006): Alleviation of soil compaction - requirements, equipment and techniques. Soil Use and Management, 22, 113-122.

Sun, Y., Schulze Lammers, P. \& Ma, D. (2004): Evaluation of a combined penetrometer for simultaneous measurement of penetration resistance and soil water content. J. Plant Nutr. Soil Sci., 167, 745-751.

Sun, Y., Ma, D., Schulze Lammers, P., Schmittmann, O. \& Rose, M. (2006): Onthe-go measurement of soil water content and mechanical resistance by a combined horizontal penetrometer. Soil Tillage Res., 86, 209-217.

To, J. \& Kay, B.D. (2005): Variation in penetrometer resistance with soil properties: the contribution of effective stress and implications for pedotransfer functions. Geoderma, 126, 261-276.

Umwelt-Geräte-Technik GmbH (UGT) (1999): Bedienungsanleitung PL 300. Müncheberg. 
Unger, P.W. \& Jones, O.R. (1998): Long-term tillage and cropping systems affect bulk density and penetration resistance of soil cropped to dryland wheat and grain sorghum. Soil Tillage Res., 45, 39-57.

United Nations (UN) (2004): World Population to 2300. New York.

van den Akker, J.J.H. \& Canarache, A. (2001): Two European concerted actions on subsoil co mpaction. Landnutzung und Landentwicklung / Land Use and Development, 42 (1), 15-22.

Verein Deutscher Ingenieure (VDI) (Hrsg.) (2007): VDI-Richtlinie 6101 Maschineneinsatz unter Berücksichtigung der Befahrbarkeit landwirtschaftlich genutzter Böden. Beuth Verlag, Berlin 2007.

Vorderbrügge, T. \& Brunotte, J. (2011): Mechanische Verdichtungsempfindlichkeit für Ackerflächen (Unterboden) - Validierung von Pedotransferfunktionen zur Ableitung der Verdichtungsempfindlichkeit bzw. zur Ausweisung "sensibler Gebiete" in Europa und ein praxisorientierter Lösungsansatz zur Guten fachlichen Praxis - Teil I: Validierung von Pedotransferfunktionen. In Johann Heinrich von Thünen-Istitut (Hrsg.): Landbauforschung - vTI Agriculture and Forestry Research, 61 (1), 1-22.

Weißbach, M. (1994): Wirkung von Fahrwerken, insbesondere im Bereich Boden/Pflanze. Dissertation Universität Kiel.

Weyer, T. \& Boeddinghaus, R. (2009): Bodenverdichtungen vermeiden Bodenfruchtbarkeit erhalten und wiederherstellen. In Ministerium für Umwelt und Naturschutz, Landwirtschaft und Verbraucherschutz des Landes Nordrhein-Westfalen (Hrsg.). Düsseldorf 2009.

Weyer, T. \& Buchner, W. (2001): Bodenschadverdichtungen - Ausmaß, Ursachen, Wirkungen und Lösungsansätze. In: Tagungsband der Fachtagung "Bodenbewirtschaftung im Umbruch", Fachhochschule Südwestfalen, Fachbereich Agrarwirtschaft, Soest, 9-31.

Whalley, W.R., To, J., Kay, B.D. \& Whitmore, A.P. (2007): Prediction of the penetrometer resistance of soils with models with few parameters. Geoderma, 137, 370-377. 
Wilde, T.A. (2000): Regeneration von Ackerböden nach starker landtechnischer Belastung. Dissertation Universität Kiel.

Würfel, T., Vetter, R., Unterseher, E. \& Elsäßer, M. (2002): Vorsorge gegen Bodenschadverdichtungen. In Landesanstalt für Pflanzenbau (Hrsg.): Merkblätter für die Umweltgerechte Landbewirtschaftung, Nr. 25. Rheinstetten 2002.

Zink, A. (2009): Bodenstabilität und Auswirkungen dynamischer Lasteinträge auf physikalische Eigenschaften von Ackerböden unter konservierender und konventioneller Bodenbearbeitung. Dissertation Universität Kiel. 


\section{Anhang}

Tabelle A 1: Temperatur und Niederschlag auf den Versuchsflächen während der Projektlaufzeit

\begin{tabular}{|c|c|c|c|c|c|c|c|c|c|c|c|c|}
\hline Termin & $\mathrm{SH}_{\text {kons }}$ & & & & $\mathrm{SH}_{\text {kon }}$ & & & & $\mathrm{NRW}_{\mathrm{k}}$ & $\mathrm{ns} / \mathrm{NRV}$ & $V_{\text {konv }}$ & \\
\hline & LJM & Nds. & LJM & Temp. & LJM & Nds. & LJM & Temp. & LJM & Nds. & LJM & Temp. \\
\hline & {$[\mathrm{mm}]$} & {$[\mathrm{mm}]$} & {$\left[{ }^{\circ} \mathrm{C}\right]$} & {$\left[{ }^{\circ} \mathrm{C}\right]$} & {$[\mathrm{mm}]$} & {$[\mathrm{mm}]$} & {$\left[{ }^{\circ} \mathrm{C}\right]$} & {$\left[{ }^{\circ} \mathrm{C}\right]$} & {$[\mathrm{mm}]$} & {$[\mathrm{mm}]$} & {$\left[{ }^{\circ} \mathrm{C}\right]$} & {$\left[{ }^{\circ} \mathrm{C}\right]$} \\
\hline $01 / 2006$ & 56,9 & 13,7 & 1,8 & $-0,6$ & 70,0 & 17,8 & 1,3 & $-0,8$ & 63,0 & 0,0 & 1,9 & $-0,2$ \\
\hline $02 / 2006$ & 49,1 & 22,4 & 2,3 & 1,6 & 45,2 & 31,6 & 1,6 & 1 & 48,0 & 36,7 & 2,3 & 1,5 \\
\hline $03 / 2006$ & 48,8 & 47,4 & 4,0 & 1,0 & 58,4 & 61,8 & 3,8 & 0,6 & 70,0 & 59,8 & 5,7 & 3,4 \\
\hline $04 / 2006$ & 41,3 & 55,0 & 8,0 & 7,1 & 41,9 & 44,7 & 7,2 & 7,1 & 51,0 & 43,6 & 8,8 & 8,8 \\
\hline 05/2006 & 62,5 & 82,6 & 12,1 & 12,0 & 49,5 & 68,2 & 11,7 & 12 & 67,0 & 99,9 & 13,4 & 14,0 \\
\hline $06 / 2006$ & 67,8 & 33,7 & 15,0 & 15,9 & 74,7 & 28,6 & 14,7 & 16,1 & 80,0 & 20,4 & 15,6 & 17,0 \\
\hline $07 / 2006$ & 100,5 & 52,9 & 17,2 & 21,2 & 85,4 & 105,8 & 16,9 & 20,9 & 78,0 & 46,1 & 18,0 & 22,1 \\
\hline $08 / 2006$ & 71,8 & 154,8 & 17,6 & 16,6 & 67,7 & 164,9 & 17,0 & 17 & 71,0 & 73,7 & 18,0 & 16,2 \\
\hline $09 / 2006$ & 60,2 & 36,6 & 14,2 & 17,0 & 72,5 & 29,4 & 13,4 & 17,3 & 72,0 & 9,9 & 14,5 & 18,1 \\
\hline $10 / 2006$ & 72,4 & 88,3 & 10,0 & 12,5 & 81,2 & 87,2 & 9,4 & 12,9 & 68,0 & 33,0 & 10,6 & 14,0 \\
\hline $11 / 2006$ & 57,4 & 65,6 & 5,1 & 7,7 & 68,3 & 55,2 & 4,8 & 7,9 & 64,0 & 63,1 & 5,5 & 9,0 \\
\hline $12 / 2006$ & 62,3 & 54,3 & 2,1 & 6,5 & 69,5 & 64,0 & 1,9 & 6,8 & 66,0 & k.A. & 2,8 & k.A. \\
\hline $01 / 2007$ & 56,9 & 142,3 & 1,8 & 5,5 & 70,0 & 156,9 & 1,3 & 5,4 & 63,0 & 99,7 & 1,9 & k.A. \\
\hline $02 / 2007$ & 49,1 & 52,8 & 2,3 & 3,7 & 45,2 & 57,8 & 1,6 & 3,5 & 48,0 & 50,6 & 2,3 & 5,6 \\
\hline $03 / 2007$ & 48,8 & 56,1 & 4,0 & 7,2 & 58,4 & 60,8 & 3,8 & 6,7 & 70,0 & 46,8 & 5,7 & 7,3 \\
\hline $04 / 2007$ & 41,3 & 2,5 & 8,0 & 10,3 & 41,9 & 2,3 & 7,2 & 10,1 & 51,0 & 1,2 & 8,8 & 11,6 \\
\hline $05 / 2007$ & 62,5 & 93,7 & 12,1 & 12,6 & 49,5 & 79,0 & 11,7 & 12,6 & 67,0 & 120,1 & 13,4 & 14,1 \\
\hline $06 / 2007$ & 67,8 & 119,8 & 15,0 & 16,3 & 74,7 & 106,3 & 14,7 & 16,6 & 80,0 & 101,6 & 15,6 & 17,2 \\
\hline $07 / 2007$ & 100,5 & 189,3 & 17,2 & 16,2 & 85,4 & 172,2 & 16,9 & 16,5 & 78,0 & 132,7 & 18,0 & 17,4 \\
\hline $08 / 2007$ & 71,8 & 58,6 & 17,6 & 17,0 & 67,7 & 50,5 & 17,0 & 17,2 & 71,0 & 154,1 & 18,0 & 17,0 \\
\hline 09/2007 & 60,2 & 70,8 & 14,2 & 13,1 & 72,5 & 65 & 13,4 & 13,5 & 72,0 & 97,4 & 14,5 & 13,6 \\
\hline $10 / 2007$ & 72,4 & 25,1 & 10,0 & 8,9 & 81,2 & 24 & 9,4 & 9,2 & 68,0 & 42,1 & 10,6 & 9,5 \\
\hline $11 / 2007$ & 57,4 & 38,0 & 5,1 & 5,0 & 68,3 & 42,2 & 4,8 & 5,1 & 64,0 & 61,2 & 5,5 & 6,2 \\
\hline $12 / 2007$ & 62,3 & 76,7 & 2,1 & 3,3 & 69,5 & 71,1 & 1,9 & 3,5 & 66,0 & 54,6 & 2,8 & 3,5 \\
\hline $01 / 2008$ & 56,9 & 64,1 & 1,8 & 4,4 & 70,0 & 67,4 & 1,3 & 4,7 & 63,0 & 54,1 & 1,9 & k.A. \\
\hline $02 / 2008$ & 49,1 & 40,2 & 2,3 & 4,7 & 45,2 & 35,3 & 1,6 & 5,1 & 48,0 & 18,5 & 2,3 & 4,4 \\
\hline $03 / 2008$ & 48,8 & 61,8 & 4,0 & 4,3 & 58,4 & 61,2 & 3,8 & 4,6 & 70,0 & 76,8 & 5,7 & 5,4 \\
\hline $04 / 2008$ & 41,3 & 40,6 & 8,0 & 7,6 & 41,9 & 42,6 & 7,2 & 7,7 & 51,0 & 74,6 & 8,8 & 8,2 \\
\hline
\end{tabular}


Tabelle A 2: Bodeneigenschaften der Versuchsfläche $\mathrm{SH}_{\text {kons }}$ (nach ZINK 2009)

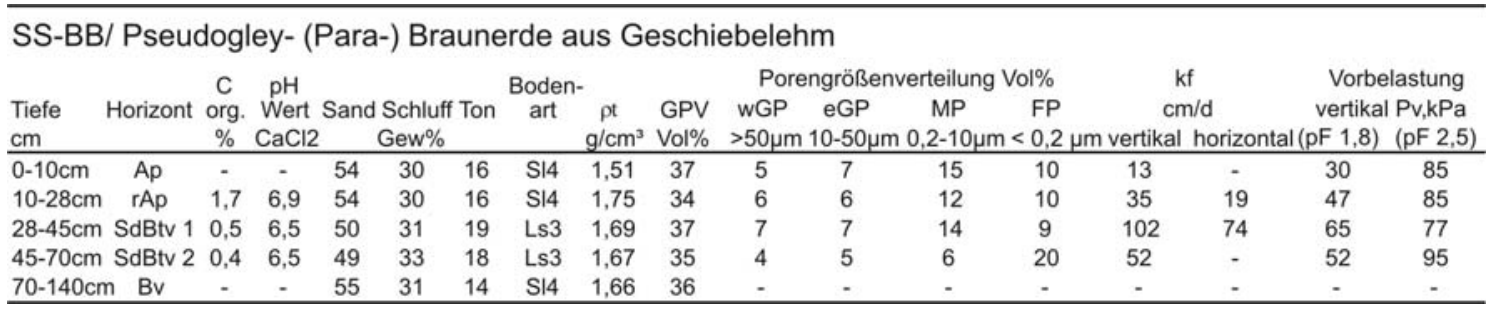

Tabelle A 3: Bodeneigenschaften der Versuchsfläche $\mathrm{SH}_{\text {konv }}$ (nach ZINK 2009)

\begin{tabular}{|c|c|c|c|c|c|c|c|c|c|c|c|c|c|c|c|c|c|}
\hline \multicolumn{18}{|c|}{ LL/ Parabraunerde aus Geschiebelehm } \\
\hline $\begin{array}{l}\text { Tiefe } \\
\mathrm{cm}\end{array}$ & Horizon & $\begin{array}{c}\mathrm{C} \\
\text { t } \underset{\mathrm{org}}{\%} \\
\text { \% }\end{array}$ & $\begin{array}{c}\mathrm{pH} \\
\text { Wert } \\
\mathrm{CaCl} 2 \\
\end{array}$ & Sand & $\begin{array}{l}\text { Schluff } \\
\text { Gew\% }\end{array}$ & Ton & $\begin{array}{l}\text { Boden- } \\
\text { art }\end{array}$ & $\begin{array}{c}\rho t \\
\mathrm{~g} / \mathrm{cm}^{3}\end{array}$ & $\begin{array}{l}\text { GPV } \\
\text { Vol\% } \\
\end{array}$ & $\begin{array}{l}\text { Pore } \\
\text { wGP } \\
>50 \mu \mathrm{m} 1\end{array}$ & $\begin{array}{l}\text { ngrößen } \\
\text { eGP } \\
10-50 \mu \mathrm{m}\end{array}$ & $\begin{array}{l}\text { iverteilung } \\
\text { MP } \\
0,2-10 \mu \mathrm{m}\end{array}$ & $\begin{array}{l}\text { Vol\% } \\
\text { FP } \\
<0,2 \mu \mathrm{m}\end{array}$ & vertikal & $\begin{array}{l}\mathrm{kf} \\
\mathrm{m} / \mathrm{d} \\
\text { I horizontal }\end{array}$ & $\begin{array}{r}\text { Vorbe } \\
\text { vertikal } \\
(\mathrm{pF} 1,8)\end{array}$ & $\begin{array}{l}\text { elastung } \\
\text { Pv, kPa } \\
\text { (pF 2,5) }\end{array}$ \\
\hline $10-28 \mathrm{~cm}$ & n Ap & 2,3 & 36,9 & 65 & 29 & 6 & Su3 & 1,58 & 40 & 13 & 5 & 9 & 13 & 57 & - & 62 & 68 \\
\hline $28-32 \mathrm{~cm}$ & n App & 2,1 & 6,5 & 63 & 28 & 9 & $\mathrm{SI} 3$ & 1,66 & 37 & 12 & 5 & 5 & 15 & 73 & 36 & 48 & 51 \\
\hline $32-50 \mathrm{~cm}$ & $n \quad B t 1$ & 0,7 & 6,1 & 53 & 33 & 14 & $\mathrm{~S} \mid 4$ & 1,75 & 34 & 6 & 4 & 3 & 21 & 126 & 10 & 57 & 68 \\
\hline$>50 \mathrm{~cm}$ & Bt2 & 0,3 & 5,9 & 51 & 31 & 18 & Ls3 & 1,71 & 35 & 5 & 3 & 7 & 20 & 20 & 12 & 45 & 61 \\
\hline
\end{tabular}

Tabelle A 4: Bodeneigenschaften der Versuchsfläche $N_{R W}$ kons (nach ZINK 2009)

\begin{tabular}{|c|c|c|c|c|c|c|c|c|c|c|c|c|c|c|c|c|c|}
\hline \multirow{2}{*}{$\begin{array}{l}\text { Tiefe } \\
\mathrm{cm}\end{array}$} & \multirow{2}{*}{\multicolumn{2}{|c|}{$\begin{array}{r}\mathrm{C} \\
\text { Horizont org. } \\
\%\end{array}$}} & \multirow{2}{*}{$\begin{array}{c}\mathrm{pH} \\
\text { Wert } \\
\mathrm{CaCl} 2 \\
\end{array}$} & \multirow{2}{*}{ Sand } & \multirow{2}{*}{$\begin{array}{l}\text { Schluff } \\
\text { Gew\% }\end{array}$} & \multirow{2}{*}{ Ton } & \multirow[t]{2}{*}{$\begin{array}{c}\text { Boden- } \\
\text { art }\end{array}$} & \multirow{2}{*}{$\begin{array}{c}\rho t \\
\mathrm{~g} / \mathrm{cm}^{3}\end{array}$} & \multirow{2}{*}{$\begin{array}{l}\text { GPV } \\
\text { Vol\% }\end{array}$} & \multicolumn{4}{|c|}{ Porengrößenverteilung Vol\% } & \multicolumn{2}{|c|}{$\begin{array}{l}\mathrm{kf} \\
\mathrm{cm} / \mathrm{d}\end{array}$} & \multicolumn{2}{|c|}{$\begin{array}{l}\text { Vorbelastung } \\
\text { vertikal } \mathrm{Pv}, \mathrm{kPa}\end{array}$} \\
\hline & & & & & & & & & & $>50 \mu \mathrm{m}$ & $10-50 \mu \mathrm{m}$ & $0,2-10 \mu \mathrm{m}$ & $<0,2 \mu \mathrm{m}$ & vertikal & horizontal ( $p$ & pF 1,8) & $(\mathrm{pF} 2,5)$ \\
\hline$\overline{0-15}$ & Ap & 2,3 & 6,6 & 3 & 81 & 16 & Ut3 & 1,48 & 44 & 1 & 2 & 29 & 12 & 63 & - & 55 & 61 \\
\hline $15-28$ & rAp & - & - & - & - & - & - & - & - & - & - & - & - & - & - & - & - \\
\hline $28-45$ & AISw & 0,8 & 6,5 & 2 & 81 & 17 & Ut3 & 1,56 & 40 & 4 & 2 & 20 & 14 & 154 & 4 & 55 & 98 \\
\hline $45-65$ & BtSd1 & 0,5 & 6,6 & 2 & 77 & 21 & Ut4 & 1,50 & 43 & 6 & 2 & 19 & 16 & 253 & 25 & 60 & 74 \\
\hline $65-135$ & 35 BtSd2 & 0,3 & 6,7 & 2 & 77 & 21 & Ut4 & 1,57 & 41 & 3 & 1 & 18 & 19 & 64 & - & 53 & 67 \\
\hline
\end{tabular}

Tabelle A 5: Bodeneigenschaften der Versuchsfläche NRW $_{\text {konv }}$ (nach ZINK 2009)

\begin{tabular}{|c|c|c|c|c|c|c|c|c|c|c|c|c|c|c|c|c|c|}
\hline \multicolumn{18}{|c|}{ SS-BB/ Pseudogley- (Para-) Braunerde aus Lößlehm } \\
\hline $\begin{array}{l}\text { Tiefe H } \\
\mathrm{cm}\end{array}$ & Horizont & $\begin{array}{c}\text { C } \\
\text { org. } \\
\%\end{array}$ & $\begin{array}{l}\mathrm{pH} \\
\text { Wert } \\
\mathrm{CaCl} 2 \\
\end{array}$ & Sand & $\begin{array}{l}\text { Schluff } \\
\text { Gew\% }\end{array}$ & Ton & $\begin{array}{c}\text { Boden- } \\
\text { art }\end{array}$ & $\begin{array}{c}\rho t \\
\mathrm{~g} / \mathrm{cm}^{3}\end{array}$ & $\begin{array}{l}\text { GPV } \\
\text { Vol\% } \\
\end{array}$ & $\begin{array}{c}\text { Pore } \\
\text { wGP } \\
>50 \mu \mathrm{m}\end{array}$ & $\begin{array}{l}\text { engrößen } \\
\text { eGP } \\
10-50 \mu \mathrm{m}\end{array}$ & $\begin{array}{c}\text { verteilung } \\
\text { MP } \\
0,2-10 \mu \mathrm{m}\end{array}$ & $\begin{array}{l}\text { Vol\% } \\
\text { FP } \\
<0,2 \text {. }\end{array}$ & 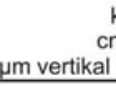 & $\begin{array}{l}\mathrm{kf} \\
\mathrm{m} / \mathrm{d} \\
\text { horizont }\end{array}$ & $\begin{array}{r}\text { Vorbe } \\
\text { vertikal } \\
\text { tal }(\mathrm{pF} 1,8) \\
\end{array}$ & $\begin{array}{l}\text { lastung } \\
\text { I Pv, kPa } \\
\text { (pF 2,5) }\end{array}$ \\
\hline $0-35$ & $A p$ & 1,9 & 6,6 & 3 & 84 & 13 & Ut3 & 1,42 & 46 & 7 & 6 & 23 & 10 & 488 & 15 & 31 & 40 \\
\hline $35-60$ & SwAIBV & $v 0,5$ & 6,9 & 3 & 84 & 13 & Ut3 & 1,57 & 40 & 3 & 5 & 20 & 12 & 103 & 39 & 75 & 58 \\
\hline $60-140$ & $0 \mathrm{SdBtv}$ & 0,5 & 6,8 & 2 & 82 & 16 & Ut3 & 1,62 & 39 & 3 & 4 & 18 & 14 & 70 & 12 & 62 & 55 \\
\hline$>140$ & Bv & - & - & - & - & - & - & - & - & - & - & - & - & - & - & - & - \\
\hline
\end{tabular}


Tabelle A 6: Bodenbearbeitung und Fruchtfolge der Versuchsflächen am Standort Nordrhein-Westfalen 2000 - 2008

\begin{tabular}{|c|c|c|c|c|}
\hline Jahr & Aussaat & vorgehende $\mathrm{BB}$ & Aussaat & vorgehende $\mathrm{BB}$ \\
\hline & NRW $_{\text {kons }}$ & $\mathrm{NRW}_{\mathrm{kons}}$ & $\underline{N R W}_{\text {Konv }}$ & $\underline{N R W}_{\text {kon }} \underline{v}$ \\
\hline \multirow[t]{3}{*}{2000} & W-Weizen & 2x Grubber & W-Gerste & $2 x$ Grubber $15 \mathrm{~cm}$ \\
\hline & & Kr.-egge $5 \mathrm{~cm}$ & & Pflug $28 \mathrm{~cm}$ \\
\hline & & & & Kr.-egge \\
\hline \multirow[t]{3}{*}{2001} & Körnererbse & SPR-egge $5 \mathrm{~cm}$ & W-Raps & $2 x$ Grubber $15 \mathrm{~cm}$ \\
\hline & & Kr.-egge $5 \mathrm{~cm}$ & & Pflug $28 \mathrm{~cm}$ \\
\hline & & & & Kr.-egge \\
\hline \multirow[t]{3}{*}{2002} & Körnermais & SPR-egge $7 \mathrm{~cm}$ & Z-Rübe & $2 x$ Grubber $15 \mathrm{~cm}$ \\
\hline & & Mulchsaat (flach) & & Pflug $28 \mathrm{~cm}$ \\
\hline & & & & Kr.-egge \\
\hline \multirow[t]{3}{*}{2003} & W-Weizen & $2 x$ Sch.-egge $8 \mathrm{~cm}$ & W-Weizen & $2 x$ Grubber $15 \mathrm{~cm}$ \\
\hline & & Sch.-egge $6 \mathrm{~cm}$ & & Pflug $28 \mathrm{~cm}$ \\
\hline & & & & Kr.-egge \\
\hline \multirow[t]{3}{*}{2004} & W-Hafer & Sch.-egge $5 \mathrm{~cm}$ & W-Weizen & Grubber $12 \mathrm{~cm}$ \\
\hline & & Universalegge $10 \mathrm{~cm}$ & & \\
\hline & & Kr.-egge $5 \mathrm{~cm}$ & & \\
\hline \multirow[t]{3}{*}{2005} & W-Gerste & $2 x$ Sch.-egge $6 \mathrm{~cm}$ & Gelbsenf & Grubber $12 \mathrm{~cm}$ \\
\hline & & Univ.-egge $6 \mathrm{~cm}$ & & \\
\hline & & Kr.-egge $6 \mathrm{~cm}$ & & \\
\hline \multirow[t]{6}{*}{2006} & Ölrettich & Grubber $8 \mathrm{~cm}$ & Ölrettich & Pflug $28 \mathrm{~cm}$ \\
\hline & & MOVIS & & MOVIS \\
\hline & & Kr.-egge $5 \mathrm{~cm}$ & & Grubbersaat ÖIrettich \\
\hline & & & & Mulchen \\
\hline & & & & MOVIS \\
\hline & & & & Grubber $12 \mathrm{~cm}$ \\
\hline \multirow[t]{6}{*}{2007} & S-Roggen & MOVIS & S-Roggen & MOVIS \\
\hline & & Grubber $12 \mathrm{~cm}(2006)$ & & Pflug $23 \mathrm{~cm}$ \\
\hline & & MOVIS & & Grubbersaat S-Roggen \\
\hline & & Grubber $8 \mathrm{~cm}(2007)$ & & Häckseln \\
\hline & & & & MOVIS \\
\hline & & & & Grubber $8 \mathrm{~cm}$ \\
\hline \multirow[t]{4}{*}{2008} & Silomais & MOVIS & Z-Rübe & MOVIS \\
\hline & & Grubber $12 \mathrm{~cm}$ & & Tiefenlockerung $35 \mathrm{~cm}$ \\
\hline & & MOVIS & & Pflug $28 \mathrm{~cm}$ \\
\hline & & Parapflug $40 \mathrm{~cm}$ & & \\
\hline
\end{tabular}


Tabelle A 7: Bodenbearbeitung und Fruchtfolge der Versuchsflächen am Standort Schleswig-Holstein $2000-2008$

\begin{tabular}{|c|c|c|c|c|}
\hline Jahr & Aussaat Ost/West & vorgehende $\mathrm{BB}$ & Aussaat & vorgehende $\mathrm{BB}$ \\
\hline & $\underline{\mathrm{SH}}_{\mathrm{kons}}$ & $\underline{\mathrm{SH}}_{\mathrm{kons}}$ & $\underline{\mathrm{SH}}_{\text {konv }}$ & $\underline{\mathrm{SH}}_{\mathrm{konv}}$ \\
\hline \multirow[t]{3}{*}{2000} & W-Raps/ W-Gerste & 2x Grubber 8cm & W-Weizen & Sch.-egge \\
\hline & & Grubber $15 \mathrm{~cm}$ & & Plug \\
\hline & & Kr.-egge & & \\
\hline \multirow[t]{3}{*}{2001} & W-Weizen/ W-Raps & 2x Grubber 8cm & Kleegras & $2 x$ Striegel \\
\hline & & Grubber $15 \mathrm{~cm}$ & & \\
\hline & & Kr.-egge & & \\
\hline \multirow[t]{3}{*}{2002} & W-Gerste/ W-Weizen & 2x Grubber 8cm & S-Hafer & 2x Sch.-egge \\
\hline & & Grubber $15 \mathrm{~cm}$ & & Pflug \\
\hline & & Kr.-egge & & \\
\hline \multirow[t]{4}{*}{2003} & W-Raps / W-Gerste & 2x Sch.-Grubber 8cm & Kartoffeln & Sch.-egge \\
\hline & & Sch.-grubber $20 \mathrm{~cm}$ & & Grubber \\
\hline & & & & Dämme aufgepflügt \\
\hline & & & & Separiert \\
\hline \multirow[t]{3}{*}{2004} & W-Weizen/ W-Raps & 2x Sch.-Grubber 8cm & Erbsen & Grubber \\
\hline & & Sch.-grubber $20 \mathrm{~cm}$ & & $\begin{array}{l}\text { Rohphosphat } \\
\text { gestreut }\end{array}$ \\
\hline & & & & Pflug \\
\hline \multirow[t]{2}{*}{2005} & W-Gerste/ W-Weizen & 2x Sch.-Grubber 8cm & S-Weizen & 3x Sch.-egge \\
\hline & & Sch.-grubber $20 \mathrm{~cm}$ & & \\
\hline \multirow[t]{3}{*}{2006} & W-Raps/ W-Gerste & $2 \times$ Sch.-egge $5 \mathrm{~cm}$ & Kleegras & Mulcher \\
\hline & & Sch.-grubber $20 \mathrm{~cm}$ & & \\
\hline & & Kr.-egge & & \\
\hline \multirow[t]{4}{*}{2007} & W-Weizen/ W-Weizen & $2 \times$ Sch.-egge $5 \mathrm{~cm}$ & S-Hafer & 2x Sch.-egge \\
\hline & & Sch.-grubber $20 \mathrm{~cm}$ & & Pflug $28 \mathrm{~cm}$ \\
\hline & & Kr.-egge & & \\
\hline & & & Kartoffeln & $2 \times$ Sch.-egge \\
\hline \multirow[t]{4}{*}{2008} & & & & 2x Grubber \\
\hline & & & & Pflug \\
\hline & & & & Dämme aufgepflügt \\
\hline & & & & Separiert \\
\hline
\end{tabular}


Tabelle A 8: Kennzahlen des verwendeten Reifens Michelin MegaXBib (Michelin 2006)

\begin{tabular}{ll}
\hline Kennzahlen MegaXBib Tubeless 650/75 R 32 & \\
\hline Reifeninhalt 75\% & 697 I \\
Größe (Vergleichsgröße) & $650 / 75$ R 32 (24.5 R 32) \\
Betriebskennung & 172 A8 (172 B) \\
verwendete Felge & DW21A \\
Breite & $657 \mathrm{~mm}$ \\
Außendurchmesser & $1825 \mathrm{~mm}$ \\
Halbmesser statisch & $821 \mathrm{~mm}$ \\
Abrollumfang & $5423 \mathrm{~mm}$ \\
Profiltiefe & $59 \mathrm{~mm}$
\end{tabular}

Tragfähigkeit (kg) pro Reifen bei Luftdruck (bar)

\begin{tabular}{|c|c|c|c|c|c|c|c|c|c|c|c|c|c|c|c|}
\hline 1,0 & 1,2 & 1,4 & 1,6 & 1,9 & 2,2 & 2,4 & 2,6 & 2,7 & 2,8 & 3,0 & 3,2 & 3,4 & 3,6 & 3,8 & $\begin{array}{l}\mathrm{km} / \\
\mathrm{h}\end{array}$ \\
\hline $\begin{array}{l}\stackrel{\infty}{\sim} \\
\text { లి }\end{array}$ & 웅 & ○ & $\begin{array}{l}\stackrel{8}{0} \\
\stackrel{\text { m}}{+}\end{array}$ & $\stackrel{\stackrel{\sim}{N}}{\underset{\gamma}{*}}$ & 융 & 유 & 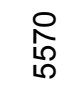 & 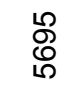 & $\frac{10}{\infty}$ & $\begin{array}{l}8 \\
8 \\
\varnothing\end{array}$ & $\begin{array}{l}\text { ○్ } \\
\text { లె }\end{array}$ & & & & 유 \\
\hline $\begin{array}{l}\stackrel{\infty}{\sim} \\
\text { లి }\end{array}$ & 웜 & ஓ̊ & $\begin{array}{l}\stackrel{8}{0} \\
\text { m }\end{array}$ & $\stackrel{\stackrel{\sim}{N}}{\underset{\gamma}{*}}$ & 융 & 尺ి & 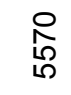 & 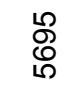 & $\underset{\infty}{\stackrel{10}{\infty}}$ & $\begin{array}{l}8 \\
8 \\
\varnothing\end{array}$ & $\begin{array}{l}\text { ○్ } \\
\text { లె }\end{array}$ & & & & ㅇ \\
\hline $\begin{array}{l}\text { 옹 } \\
\text { ్ㅐ }\end{array}$ & 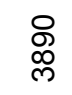 & $\begin{array}{l}\stackrel{\bigcirc}{\mathscr{N}} \\
\text { † }\end{array}$ & 옹 & $\begin{array}{l}0 \\
\varnothing \\
0 \\
0\end{array}$ & 尔 & $\begin{array}{l}0 \\
\text { م }\end{array}$ & 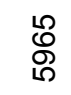 & 용 & ్ํำ & $\begin{array}{l}\circ \\
\text { ఫீ }\end{array}$ & 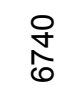 & & & & ஜ \\
\hline $\begin{array}{l}8 \\
\stackrel{\circ}{\checkmark}\end{array}$ & $\begin{array}{l}\stackrel{8}{\infty} \\
\stackrel{9}{+}\end{array}$ & $\begin{array}{l}8 \\
\qquad \\
\dot{0}\end{array}$ & 옴 & ণ্ণ & $\begin{array}{l}\text { గి } \\
\infty \\
0\end{array}$ & $\frac{n}{\nwarrow}$ & $\begin{array}{l}\text { חొ } \\
\stackrel{1}{N}\end{array}$ & 足 & 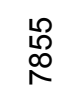 & $\frac{n}{\infty}$ & Љ̊ & $\underset{\infty}{\stackrel{0}{\infty}}$ & $\frac{\text { లో }}{\sigma}$ & $\begin{array}{l}\text { 웅 } \\
\text { \& }\end{array}$ & 음 \\
\hline ஓి & 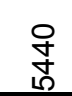 & $\begin{array}{l}\circ \\
\infty \\
0 \\
0\end{array}$ & $\begin{array}{l}\text { స్ } \\
\text { గ్ర } \\
\end{array}$ & 옹 & $\begin{array}{l}0 \\
\mathbb{0} \\
\\
\end{array}$ & 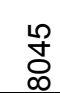 & $\stackrel{\stackrel{\sim}{\mathcal{W}}}{\underset{\infty}{\infty}}$ & $\sum_{\infty}^{\infty}$ & $\begin{array}{l}10 \\
\infty \\
\infty \\
\infty\end{array}$ & $\frac{10}{\infty}$ & $\begin{array}{l}n \\
0 \\
0 \\
0\end{array}$ & 융 & 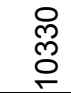 & 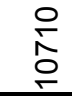 & 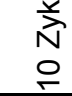 \\
\hline
\end{tabular}


Tabelle A 9: Gravimetrischer Wassergehalt [\%] nach Fläche, Messtiefe und Untersuchungstermin

\begin{tabular}{|c|c|c|c|c|c|c|c|c|}
\hline Fläche & Termin & Tiefe $[\mathrm{cm}]$ & Chronologie & $\mathrm{n}$ & $M$ & SD & SED & GD5\% \\
\hline \multirow[t]{24}{*}{$\mathrm{SH}_{\text {kons }}$} & \multirow{6}{*}{$\begin{array}{l}\text { Herbst } \\
2006\end{array}$} & \multirow[t]{2}{*}{0} & vor Bef. & 20 & 20,4 & 2,3 & 0,5 & 1,4 \\
\hline & & & nach Bef. & 20 & 21,9 & 1,6 & 0,4 & 1,0 \\
\hline & & \multirow[t]{2}{*}{20} & vor Bef. & 20 & 15,6 & 1,4 & 0,3 & 0,9 \\
\hline & & & nach Bef. & 20 & 17,4 & 1,2 & 0,3 & 0,8 \\
\hline & & \multirow[t]{2}{*}{40} & vor Bef. & 20 & 17,2 & 1,4 & 0,3 & 0,9 \\
\hline & & & nach Bef. & 20 & 17,4 & 0,5 & 0,1 & 0,3 \\
\hline & \multirow{6}{*}{$\begin{array}{l}\text { Frühjahr } \\
2007\end{array}$} & \multirow[t]{2}{*}{0} & vor Bef. & 20 & 10,0 & 1,5 & 0,3 & 0,9 \\
\hline & & & nach Bef. & 20 & 10,7 & 1,8 & 0,4 & 1,1 \\
\hline & & \multirow[t]{2}{*}{20} & vor Bef. & 19 & 12,5 & 1,4 & 0,3 & 0,9 \\
\hline & & & nach Bef. & 20 & 13,0 & 1,4 & 0,3 & 0,9 \\
\hline & & \multirow[t]{2}{*}{40} & vor Bef. & 15 & 15,5 & 1,4 & 0,4 & 1,0 \\
\hline & & & nach Bef. & 20 & 15,7 & 2,2 & 0,5 & 1,4 \\
\hline & \multirow{6}{*}{$\begin{array}{l}\text { Herbst } \\
2007\end{array}$} & \multirow[t]{2}{*}{0} & vor Bef. & 20 & 19,2 & 1,6 & 0,4 & 1,0 \\
\hline & & & nach Bef. & 20 & 17,6 & 1,9 & 0,4 & 1,2 \\
\hline & & \multirow[t]{2}{*}{20} & vor Bef. & 20 & 16,0 & 0,9 & 0,2 & 0,6 \\
\hline & & & nach Bef. & 20 & 16,0 & 0,9 & 0,2 & 0,6 \\
\hline & & \multirow[t]{2}{*}{40} & vor Bef. & 20 & 17,1 & 1,1 & 0,2 & 0,7 \\
\hline & & & nach Bef. & 20 & 17,1 & 1,1 & 0,2 & 0,7 \\
\hline & \multirow{6}{*}{$\begin{array}{l}\text { Frühjahr } \\
2008\end{array}$} & \multirow[t]{2}{*}{0} & vor Bef. & 20 & 21,6 & 1,2 & 0,3 & 0,8 \\
\hline & & & nach Bef. & 20 & 20,7 & 1,0 & 0,2 & 0,6 \\
\hline & & \multirow[t]{2}{*}{20} & vor Bef. & 20 & 15,9 & 1,1 & 0,2 & 0,7 \\
\hline & & & nach Bef. & 20 & 14,6 & 0,8 & 0,2 & 0,5 \\
\hline & & \multirow[t]{2}{*}{40} & vor Bef. & 20 & 17,4 & 1,5 & 0,3 & 0,9 \\
\hline & & & nach Bef. & 20 & 16,0 & 0,8 & 0,2 & 0,5 \\
\hline \multirow[t]{12}{*}{$\mathrm{SH}_{\mathrm{konv}}$} & \multirow{6}{*}{$\begin{array}{l}\text { Herbst } \\
2006\end{array}$} & \multirow[t]{2}{*}{0} & vor Bef. & 20 & 18,2 & 1,3 & 0,3 & 0,8 \\
\hline & & & nach Bef. & 20 & 15,1 & 1,4 & 0,3 & 0,8 \\
\hline & & \multirow[t]{2}{*}{20} & vor Bef. & 20 & 15,9 & 1,5 & 0,3 & 0,9 \\
\hline & & & nach Bef. & 20 & 14,7 & 1,7 & 0,4 & 1,1 \\
\hline & & \multirow[t]{2}{*}{40} & vor Bef. & 20 & 15,2 & 2,8 & 0,6 & 1,7 \\
\hline & & & nach Bef. & 20 & 13,5 & 1,9 & 0,4 & 1,2 \\
\hline & \multirow{6}{*}{$\begin{array}{l}\text { Frühjahr } \\
2007\end{array}$} & \multirow[t]{2}{*}{0} & vor Bef. & 20 & 8,0 & 1,0 & 0,2 & 0,6 \\
\hline & & & nach Bef. & 20 & 7,2 & 1,0 & 0,2 & 0,6 \\
\hline & & \multirow[t]{2}{*}{20} & vor Bef. & 20 & 12,3 & 1,6 & 0,4 & 1,0 \\
\hline & & & nach Bef. & 20 & 13,9 & 1,0 & 0,2 & 0,6 \\
\hline & & \multirow[t]{2}{*}{40} & vor Bef. & 20 & 13,9 & 2,6 & 0,6 & 1,6 \\
\hline & & & nach Bef. & 20 & 14,4 & 1,3 & 0,3 & 0,8 \\
\hline
\end{tabular}




\begin{tabular}{|c|c|c|c|c|c|c|c|c|}
\hline & \multirow{6}{*}{$\begin{array}{l}\text { Herbst } \\
2007\end{array}$} & \multirow[t]{2}{*}{0} & vor Bef. & 20 & 14,0 & 1,2 & 0,3 & 0,7 \\
\hline & & & nach Bef. & 20 & 14,0 & 1,2 & 0,3 & 0,7 \\
\hline & & \multirow[t]{2}{*}{20} & vor Bef. & 20 & 14,7 & 1,0 & 0,2 & 0,6 \\
\hline & & & nach Bef. & 20 & 14,7 & 1,0 & 0,2 & 0,6 \\
\hline & & \multirow[t]{2}{*}{40} & vor Bef. & 20 & 13,5 & 0,9 & 0,2 & 0,6 \\
\hline & & & nach Bef. & 20 & 13,5 & 0,9 & 0,2 & 0,6 \\
\hline & \multirow{6}{*}{$\begin{array}{l}\text { Frühjahr } \\
2008\end{array}$} & \multirow[t]{2}{*}{0} & vor Bef. & 20 & 16,9 & 1,1 & 0,2 & 0,7 \\
\hline & & & nach Bef. & 20 & 15,1 & 1,3 & 0,3 & 0,8 \\
\hline & & \multirow[t]{2}{*}{20} & vor Bef. & 20 & 15,2 & 0,9 & 0,2 & 0,6 \\
\hline & & & nach Bef. & 20 & 14,7 & 1,3 & 0,3 & 0,8 \\
\hline & & \multirow[t]{2}{*}{40} & vor Bef. & 20 & 14,9 & 1,4 & 0,3 & 0,9 \\
\hline & & & nach Bef. & 20 & 13,9 & 2,2 & 0,5 & 1,4 \\
\hline \multirow[t]{23}{*}{$\mathrm{NRW}_{\text {kons }}$} & \multirow{6}{*}{$\begin{array}{l}\text { Frühjahr } \\
2006\end{array}$} & \multirow[t]{2}{*}{0} & vor Bef. & 20 & 23,1 & 2,8 & 0,6 & 1,7 \\
\hline & & & nach Bef. & 20 & 22,7 & 2,9 & 0,7 & 1,8 \\
\hline & & \multirow[t]{2}{*}{20} & vor Bef. & 20 & 23,3 & 1,6 & 0,4 & 1,0 \\
\hline & & & nach Bef. & 20 & 24,5 & 0,9 & 0,2 & 0,5 \\
\hline & & \multirow[t]{2}{*}{40} & vor Bef. & 20 & 24,4 & 0,9 & 0,2 & 0,6 \\
\hline & & & nach Bef. & 20 & 24,1 & 0,7 & 0,1 & 0,4 \\
\hline & \multirow{5}{*}{$\begin{array}{l}\text { Herbst } \\
2006\end{array}$} & \multirow[t]{2}{*}{0} & vor Bef. & 20 & 24,1 & 1,8 & 0,4 & 1,1 \\
\hline & & & nach Bef. & 20 & 22,4 & 1,9 & 0,4 & 1,2 \\
\hline & & \multirow[t]{2}{*}{20} & vor Bef. & 20 & 22,7 & 1,4 & 0,3 & 0,9 \\
\hline & & & nach Bef. & 20 & 22,6 & 1,1 & 0,2 & 0,7 \\
\hline & & 40 & vor Bef. & 20 & 24,3 & 0,9 & 0,2 & 0,5 \\
\hline & \multirow{6}{*}{$\begin{array}{l}\text { Frühjahr } \\
2007\end{array}$} & \multirow[t]{2}{*}{0} & vor Bef. & 20 & 16,4 & 2,4 & 0,5 & 1,5 \\
\hline & & & nach Bef. & 20 & 14,3 & 3,0 & 0,7 & 1,8 \\
\hline & & \multirow[t]{2}{*}{20} & vor Bef. & 20 & 21,7 & 0,8 & 0,2 & 0,5 \\
\hline & & & nach Bef. & 20 & 21,0 & 1,3 & 0,3 & 0,8 \\
\hline & & \multirow[t]{2}{*}{40} & vor Bef. & 20 & 22,6 & 1,1 & 0,2 & 0,7 \\
\hline & & & nach Bef. & 20 & 22,3 & 0,3 & 0,1 & 0,2 \\
\hline & \multirow{6}{*}{$\begin{array}{l}\text { Herbst } \\
2007\end{array}$} & \multirow[t]{2}{*}{0} & vor Bef. & 20 & 23,7 & 1,6 & 0,4 & 1,0 \\
\hline & & & nach Bef. & 20 & 25,8 & 1,3 & 0,3 & 0,8 \\
\hline & & \multirow[t]{2}{*}{20} & vor Bef. & 20 & 21,9 & 0,8 & 0,2 & 0,5 \\
\hline & & & nach Bef. & 20 & 22,8 & 0,7 & 0,2 & 0,4 \\
\hline & & \multirow[t]{2}{*}{40} & vor Bef. & 20 & 23,4 & 0,9 & 0,2 & 0,6 \\
\hline & & & nach Bef. & 20 & 23,4 & 0,5 & 0,1 & 0,3 \\
\hline
\end{tabular}




\begin{tabular}{|c|c|c|c|c|c|c|c|c|}
\hline & Frühjahr & 0 & vor Bef. & 20 & 24,8 & 2,1 & 0,5 & 1,3 \\
\hline & 2008 & & nach Bef. & 10 & 23,9 & 1,8 & 0,6 & 1,5 \\
\hline & & 20 & vor Bef. & 20 & 21,4 & 1,1 & 0,3 & 0,7 \\
\hline & & & nach Bef. & 10 & 21,2 & 0,8 & 0,2 & 0,7 \\
\hline & & 40 & vor Bef. & 20 & 23,2 & 0,9 & 0,2 & 0,5 \\
\hline & & & nach Bef. & 10 & 22,4 & 0,5 & 0,2 & 0,4 \\
\hline $\mathrm{NRW}_{\text {konv }}$ & Frühjahr & 0 & vor Bef. & 20 & 10,9 & 1,2 & 0,3 & 0,8 \\
\hline & & & nach Bef. & 20 & 13,5 & 2,3 & 0,5 & 1,4 \\
\hline & & 20 & vor Bef. & 20 & 20,4 & 2,1 & 0,5 & 1,3 \\
\hline & & & nach Bef. & 20 & 21,7 & 2,3 & 0,5 & 1,4 \\
\hline & & 40 & vor Bef. & 20 & 22,1 & 0,7 & 0,2 & 0,4 \\
\hline & & & nach Bef. & 20 & 21,1 & 0,8 & 0,2 & 0,5 \\
\hline & Herbst & 0 & vor Bef. & 20 & 24,6 & 1,3 & 0,3 & 0,8 \\
\hline & & & nach Bef. & 20 & 23,4 & 2,0 & 0,4 & 1,2 \\
\hline & & 20 & vor Bef. & 20 & 23,7 & 1,8 & 0,4 & 1,1 \\
\hline & & & nach Bef. & 20 & 22,0 & 1,7 & 0,4 & 1,1 \\
\hline & & 40 & vor Bef. & 20 & 22,6 & 0,9 & 0,2 & 0,6 \\
\hline & & & nach Bef. & 20 & 21,2 & 1,6 & 0,4 & 1,0 \\
\hline & Frühjahr & 0 & vor Bef. & 20 & 13,9 & 2,2 & 0,5 & 1,4 \\
\hline & & & nach Bef. & 20 & 13,1 & 1,9 & 0,4 & 1,2 \\
\hline & & 20 & vor Bef. & 20 & 22,6 & 0,8 & 0,2 & 0,5 \\
\hline & & & nach Bef. & 20 & 20,9 & 1,4 & 0,3 & 0,8 \\
\hline & & 40 & vor Bef. & 20 & 21,5 & 0,7 & 0,2 & 0,4 \\
\hline & & & nach Bef. & 20 & 21,2 & 0,9 & 0,2 & 0,6 \\
\hline & Herbst & 0 & vor Bef. & 20 & 23,0 & 0,9 & 0,2 & 0,6 \\
\hline & & & nach Bef. & 20 & 22,7 & 1,7 & 0,4 & 1,1 \\
\hline & & 20 & vor Bef. & 20 & 24,3 & 0,8 & 0,2 & 0,5 \\
\hline & & & nach Bef. & 20 & 23,8 & 1,0 & 0,2 & 0,6 \\
\hline & & 40 & vor Bef. & 20 & 21,9 & 0,8 & 0,2 & 0,5 \\
\hline & & & nach Bef. & 20 & 22,0 & 0,8 & 0,2 & 0,5 \\
\hline & Frühjahr & 0 & vor Bef. & 20 & 23,6 & 1,2 & 0,3 & 0,7 \\
\hline & & & nach Bef. & 10 & 22,3 & 0,9 & 0,3 & 0,8 \\
\hline & & 20 & vor Bef. & 20 & 22,7 & 0,9 & 0,2 & 0,6 \\
\hline & & & nach Bef. & 10 & 23,0 & 0,7 & 0,2 & 0,6 \\
\hline & & 40 & vor Bef. & 20 & 21,9 & 0,9 & 0,2 & 0,6 \\
\hline & & & nach Bef. & 10 & 22,9 & 1,7 & 0,5 & 1,5 \\
\hline
\end{tabular}


Tabelle A 10: Mittlere Kontaktflächendrücke [kPa] der Belastungsvarianten nach Termin und Fläche

\begin{tabular}{|c|c|c|c|c|c|}
\hline \multirow[t]{2}{*}{ Variante } & \multirow[t]{2}{*}{ Termin } & \multicolumn{4}{|c|}{ Mittlere Kontaktflächendrücke [kPa] } \\
\hline & & $\underline{\mathrm{SH}}_{\mathrm{kons}}$ & $\underline{\mathrm{SH}}_{\mathrm{konv}}$ & $\underline{N R W}_{\text {kons }}$ & $\underline{N R W}_{\text {konv }}$ \\
\hline \multirow[t]{5}{*}{ 3,3Mg@50kPa } & Frühjahr 2006 & . & . & 54,5 & 80,8 \\
\hline & Herbst 2006 & 55,5 & 255,7 & 70,8 & 109,8 \\
\hline & Frühjahr 2007 & 253,7 & 99,3 & 210,3 & . \\
\hline & Herbst 2007 & 67,1 & 101,7 & 104,0 & 222,6 \\
\hline & Frühjahr 2008 & 77,7 & 255,1 & 74,1 & 75,0 \\
\hline \multirow[t]{5}{*}{ 3,3Mg@160kPa } & Frühjahr 2006 & . & . & 79,3 & 120,1 \\
\hline & Herbst 2006 & 114,4 & 355,0 & 75,6 & 173,9 \\
\hline & Frühjahr 2007 & 450,2 & 107,1 & 268,8 & 331,2 \\
\hline & Herbst 2007 & 116,5 & 123,4 & 139,8 & 319,5 \\
\hline & Frühjahr 2008 & 99,9 & 402,4 & 103,1 & 102,8 \\
\hline \multirow[t]{5}{*}{ 6,3Mg@160kPa } & Frühjahr 2006 & & & 93,8 & 149,7 \\
\hline & Herbst 2006 & 120,0 & 466,1 & 168,7 & 141,8 \\
\hline & Frühjahr 2007 & 540,3 & 144,1 & 209,0 & 386,4 \\
\hline & Herbst 2007 & 129,3 & 126,8 & 171,7 & 175,0 \\
\hline & Frühjahr 2008 & 150,6 & 156,5 & 137,1 & 133,3 \\
\hline \multirow[t]{5}{*}{ 6,3Mg@250kPa } & Frühjahr 2006 & . & . & 117,2 & 157,9 \\
\hline & Herbst 2006 & 182,5 & 467,7 & 230,4 & 265,7 \\
\hline & Frühjahr 2007 & 569,9 & 190,1 & 526,0 & 451,9 \\
\hline & Herbst 2007 & 154,9 & 194,9 & 200,7 & 174,3 \\
\hline & Frühjahr 2008 & 160,5 & 168,9 & 146,0 & 170,7 \\
\hline \multirow[t]{5}{*}{ 7,5Mg@250kPa } & Frühjahr 2006 & & . & . & . \\
\hline & Herbst 2006 & 164,4 & 523,7 & 156,6 & 174,4 \\
\hline & Frühjahr 2007 & 591,7 & 182,8 & 267,5 & 445,0 \\
\hline & Herbst 2007 & 156,0 & 122,7 & 221,4 & 221,1 \\
\hline & Frühjahr 2008 & 190,7 & 226,3 & 166,1 & 203,1 \\
\hline \multirow[t]{5}{*}{ 7,5Mg@350kPa } & Frühjahr 2006 & . & . & . & . \\
\hline & Herbst 2006 & 197,4 & 872,3 & 173,3 & 186,8 \\
\hline & Frühjahr 2007 & 654,6 & 201,0 & 287,4 & 479,1 \\
\hline & Herbst 2007 & 183,5 & 241,0 & . & 198,9 \\
\hline & Frühjahr 2008 & 201,5 & 232,1 & 173,5 & 209,9 \\
\hline
\end{tabular}


Tabelle A 11: Mittelwerte der gemessenen Spurtiefen (ohne Variante nur Schlepper) nach Fläche, Radlast (RL) und Reifeninnendruck (RID) gemittelt über den Versuchszeitraum

\begin{tabular}{|c|c|c|c|c|c|c|c|}
\hline Fläche & $\mathrm{RL}[\mathrm{Mg}]$ & $\mathrm{RID}$ [kPa] & Spurtiefe & $\mathrm{n}$ & SD & SED & $\mathrm{GD}_{5 \%}$ \\
\hline \multirow[t]{6}{*}{$\mathrm{NRW}_{\text {kons }}$} & 3,3 & 50 & $-3,2$ & 81 & 0,99 & 0,110 & 0,30 \\
\hline & & 160 & $-4,1$ & 81 & 0,85 & 0,095 & \\
\hline & 6,3 & 160 & $-4,6$ & 81 & 0,94 & 0,104 & 0,29 \\
\hline & & 250 & $-5,1$ & 81 & 0,82 & 0,091 & \\
\hline & 7,5 & 250 & $-4,8$ & 72 & 1,62 & 0,191 & 0,53 \\
\hline & & 350 & $-5,9$ & 126 & 0,90 & 0,080 & \\
\hline \multirow[t]{6}{*}{$\mathrm{NRW}_{\text {konv }}$} & 3,3 & 50 & $-3,1$ & 117 & 0,64 & 0,059 & 0,16 \\
\hline & & 160 & $-4,5$ & 117 & 0,75 & 0,069 & \\
\hline & 6,3 & 160 & $-5,1$ & 117 & 0,94 & 0,087 & 0,24 \\
\hline & & 250 & $-5,7$ & 117 & 0,90 & 0,084 & \\
\hline & 7,5 & 250 & $-5,9$ & 108 & 1,45 & 0,139 & 0,39 \\
\hline & & 350 & $-6,9$ & 108 & 1,68 & 0,162 & \\
\hline \multirow[t]{6}{*}{$\mathrm{SH}_{\text {kons }}$} & 3,3 & 50 & $-3,3$ & 60 & 1,14 & 0,147 & 0,41 \\
\hline & & 160 & $-4,2$ & 60 & 1,44 & 0,186 & \\
\hline & 6,3 & 160 & $-4,3$ & 60 & 1,39 & 0,179 & 0,50 \\
\hline & & 250 & $-4,8$ & 60 & 1,25 & 0,162 & \\
\hline & 7,5 & 250 & $-4,8$ & 60 & 1,58 & 0,204 & 0,57 \\
\hline & & 350 & $-5,2$ & 87 & 1,96 & 0,210 & \\
\hline \multirow[t]{6}{*}{$\mathrm{SH}_{\text {konv }}$} & 3,3 & 50 & $-3,8$ & 60 & 1,43 & 0,185 & 0,51 \\
\hline & & 160 & $-4,7$ & 60 & 1,73 & 0,224 & \\
\hline & 6,3 & 160 & $-4,8$ & 60 & 1,66 & 0,215 & 0,60 \\
\hline & & 250 & $-5,0$ & 60 & 2,06 & 0,265 & \\
\hline & 7,5 & 250 & $-5,3$ & 60 & 1,66 & 0,215 & 0,60 \\
\hline & & 350 & $-6,1$ & 87 & 1,85 & 0,199 & \\
\hline
\end{tabular}


Tabelle A 12: Mittelwerte der gemessenen Spurtiefen bei verschiedenen Radlasten (RL) in Abhängigkeit der angewendeten Bodenbearbeitung (BB)

\begin{tabular}{lllllll}
\hline $\mathrm{RL}[\mathrm{Mg}]$ & $\mathrm{BB}$ & $\mathrm{n}$ & $\mathrm{M}[\mathrm{cm}]$ & $\mathrm{SD}$ & $\mathrm{SED}$ & GD $_{5 \%}$ \\
\hline 3,3 & kons. & 282 & $-3,69$ & 1,19 & 0,07 & 0,20 \\
& konv. & 354 & $-3,96$ & 1,27 & 0,07 & 0,19 \\
6,3 & kons. & 282 & $-4,68$ & 1,12 & 0,07 & 0,19 \\
& konv. & 354 & $-5,24$ & 1,36 & 0,07 & 0,20 \\
7,5 & kons. & 345 & $-5,33$ & 1,57 & 0,08 & 0,23 \\
& konv. & 363 & $-6,14$ & 1,75 & 0,09 & 0,25 \\
\hline
\end{tabular}

Tabelle A 13: Mittelwerte der gemessenen Spurtiefen bei verschiedenen Radlasten (RL) in Abhängigkeit des eingestellten Reifeninnendruckes (RID)

\begin{tabular}{lllllll}
\hline $\mathrm{RL}[\mathrm{Mg}]$ & $\mathrm{RID}[\mathrm{kPa}]$ & $\mathrm{n}$ & $\mathrm{M}[\mathrm{cm}]$ & $\mathrm{SD}$ & $\mathrm{SED}$ & $\mathrm{GD}_{5 \%}$ \\
\hline 3,3 & 50 & 318 & $-3,30$ & 1,05 & 0,06 & 0,16 \\
& 160 & 318 & $-4,38$ & 1,17 & 0,07 & 0,18 \\
6,3 & 160 & 318 & $-4,74$ & 1,23 & 0,07 & 0,19 \\
& 250 & 318 & $-5,24$ & 1,30 & 0,07 & 0,20 \\
7,5 & 250 & 300 & $-5,29$ & 1,62 & 0,09 & 0,26 \\
& 350 & 408 & $-6,08$ & 1,70 & 0,08 & 0,23 \\
\hline
\end{tabular}


Tabelle A 14: Mittelwerte der vertikalen Eindringwiderstände nach Fläche und Messtiefenbereich (MTB) vor Versuchsbeginn

\begin{tabular}{lllllll}
\hline Fläche & MTB [cm] & $\mathrm{n}$ & $\mathrm{M}$ & $\mathrm{SD}$ & $\mathrm{SED}$ & GD $_{5 \%}$ \\
\hline NRW $_{\text {kons }}$ & $5-14$ & 90 & 1,27 & 0,35 & 0,04 & 0,10 \\
& $18-27$ & 90 & 1,68 & 0,40 & 0,04 & 0,12 \\
& $30-39$ & 90 & 1,58 & 0,47 & 0,05 & 0,14 \\
& $50-59$ & 90 & 1,14 & 0,37 & 0,04 & 0,11 \\
NRW $_{\text {konv }}$ & $5-14$ & 120 & 0,58 & 0,24 & 0,02 & 0,06 \\
& $18-27$ & 120 & 0,63 & 0,26 & 0,02 & 0,07 \\
& $30-39$ & 120 & 1,62 & 0,74 & 0,07 & 0,19 \\
& $50-59$ & 120 & 1,85 & 0,62 & 0,06 & 0,16 \\
SH $_{\text {kons }}$ & $5-14$ & 80 & 1,22 & 0,41 & 0,05 & 0,13 \\
& $18-27$ & 80 & 2,36 & 0,61 & 0,07 & 0,19 \\
& $30-39$ & 80 & 2,26 & 0,77 & 0,09 & 0,24 \\
& $50-59$ & 80 & 2,04 & 0,86 & 0,10 & 0,27 \\
SH $_{\text {konv }}$ & $5-14$ & 96 & 2,46 & 0,62 & 0,06 & 0,18 \\
& $18-27$ & 96 & 3,04 & 0,90 & 0,09 & 0,25 \\
& $30-39$ & 96 & 4,42 & 1,18 & 0,12 & 0,33 \\
& $50-59$ & 95 & 4,06 & 1,39 & 0,14 & 0,40 \\
\hline
\end{tabular}


Tabelle A 15: Mittelwerte der vertikalen Eindringwiderstände nach Fläche und Variante vor Versuchsbeginn

\begin{tabular}{|c|c|c|c|c|c|c|}
\hline Fläche & Variante & $\mathrm{n}$ & M & SD & SED & $\mathrm{GD}_{5 \%}$ \\
\hline \multirow[t]{8}{*}{$\mathrm{SH}_{\text {kons }}$} & Kontrolle & 60 & 2,20 & 1,03 & 0,13 & 0,37 \\
\hline & 3,3Mg@50kPa & 40 & 2,19 & 0,99 & 0,16 & 0,43 \\
\hline & 3,3Mg@160kPa & 40 & 1,94 & 0,78 & 0,12 & 0,34 \\
\hline & 6,3Mg@160kPa & 40 & 1,65 & 0,52 & 0,08 & 0,23 \\
\hline & 6,3Mg@250kPa & 40 & 1,96 & 0,76 & 0,12 & 0,33 \\
\hline & 7,5Mg@250kPa & 40 & 2,11 & 0,70 & 0,11 & 0,31 \\
\hline & 7,5Mg@350kPa & 60 & 1,76 & 0,65 & 0,08 & 0,23 \\
\hline & Insgesamt & 320 & 1,97 & 0,82 & 0,05 & 0,13 \\
\hline \multirow[t]{8}{*}{$\mathrm{SH}_{\text {konv }}$} & Kontrolle & 72 & 3,54 & 1,22 & 0,14 & 0,40 \\
\hline & 3,3Mg@50kPa & 48 & 3,56 & 1,47 & 0,21 & 0,59 \\
\hline & 3,3Mg@160kPa & 48 & 2,93 & 0,94 & 0,14 & 0,38 \\
\hline & 6,3Mg@160kPa & 48 & 3,06 & 1,20 & 0,17 & 0,48 \\
\hline & 6,3Mg@250kPa & 48 & 3,58 & 1,37 & 0,20 & 0,55 \\
\hline & 7,5Mg@250kPa & 47 & 4,06 & 1,42 & 0,21 & 0,57 \\
\hline & 7,5Mg@350kPa & 72 & 3,63 & 1,33 & 0,16 & 0,44 \\
\hline & Insgesamt & 383 & 3,49 & 1,32 & 0,07 & 0,19 \\
\hline \multirow[t]{8}{*}{ NRW $W_{\text {kons }}$} & Kontrolle & 80 & 1,40 & 0,45 & 0,05 & 0,14 \\
\hline & 3,3Mg@50kPa & 40 & 1,42 & 0,49 & 0,08 & 0,22 \\
\hline & 3,3Mg@160kPa & 40 & 1,35 & 0,51 & 0,08 & 0,22 \\
\hline & 6,3Mg@160kPa & 40 & 1,37 & 0,45 & 0,07 & 0,20 \\
\hline & 6,3Mg@250kPa & 40 & 1,39 & 0,42 & 0,07 & 0,19 \\
\hline & 7,5Mg@250kPa & 40 & 1,51 & 0,43 & 0,07 & 0,19 \\
\hline & 7,5Mg@350kPa & 80 & 1,45 & 0,45 & 0,05 & 0,14 \\
\hline & Insgesamt & 360 & 1,42 & 0,45 & 0,02 & 0,07 \\
\hline \multirow[t]{9}{*}{ NRW $W_{\text {konv }}$} & Kontrolle & 60 & 1,22 & 0,77 & 0,10 & 0,27 \\
\hline & nur Schlepper & 60 & 1,07 & 0,71 & 0,09 & 0,25 \\
\hline & 3,3Mg@50kPa & 60 & 1,11 & 0,71 & 0,09 & 0,25 \\
\hline & 3,3Mg@160kPa & 60 & 1,22 & 0,89 & 0,12 & 0,32 \\
\hline & 6,3Mg@160kPa & 60 & 1,16 & 0,81 & 0,10 & 0,29 \\
\hline & 6,3Mg@250kPa & 60 & 1,14 & 0,66 & 0,09 & 0,24 \\
\hline & 7,5Mg@250kPa & 60 & 1,24 & 0,82 & 0,11 & 0,29 \\
\hline & 7,5Mg@350kPa & 60 & 1,18 & 0,79 & 0,10 & 0,28 \\
\hline & Insgesamt & 480 & 1,17 & 0,77 & 0,04 & 0,10 \\
\hline
\end{tabular}


Tabelle A 16: Effekte einer Befahrung auf den vertikalen Eindringwiderstand nach zuvor erfolgter Bodenbearbeitung

\begin{tabular}{|c|c|c|c|c|c|c|c|c|}
\hline $\begin{array}{l}\text { Fläche/ } \\
\text { Termin }\end{array}$ & MTB [cm] & Variante & Chronologie & $\mathrm{n}$ & M & SD & SED & GD5\% \\
\hline \multirow[t]{42}{*}{ SH konv. } & $5-14$ & Kontrolle & vor Bef. & 18 & 1,27 & 0,45 & 0,11 & 0,29 \\
\hline & & & nach Bef. & 18 & 1,35 & 0,49 & 0,12 & 0,32 \\
\hline & & 3,3Mg@50kPa & vor Bef. & 12 & 1,11 & 0,51 & 0,15 & 0,41 \\
\hline & & & nach Bef. & 12 & 1,57 & 0,26 & 0,07 & 0,20 \\
\hline & & 3,3Mg@160kPa & vor Bef. & 12 & 0,98 & 0,24 & 0,07 & 0,20 \\
\hline & & & nach Bef. & 12 & 1,77 & 0,55 & 0,16 & 0,44 \\
\hline & & 6,3Mg@160kPa & vor Bef. & 12 & 1,19 & 0,54 & 0,15 & 0,43 \\
\hline & & & nach Bef. & 12 & 1,65 & 0,24 & 0,07 & 0,19 \\
\hline & & 6,3Mg@250kPa & vor Bef. & 12 & 0,97 & 0,37 & 0,11 & 0,29 \\
\hline & & & nach Bef. & 12 & 1,58 & 0,34 & 0,10 & 0,27 \\
\hline & & 7,5Mg@250kPa & vor Bef. & 12 & 0,99 & 0,31 & 0,09 & 0,25 \\
\hline & & & nach Bef. & 12 & 1,93 & 0,47 & 0,14 & 0,37 \\
\hline & & 7,5Mg@350kPa & vor Bef. & 18 & 1,12 & 0,38 & 0,09 & 0,25 \\
\hline & & & nach Bef. & 18 & 1,59 & 0,27 & 0,06 & 0,18 \\
\hline & $18-27$ & Kontrolle & vor Bef. & 18 & 1,37 & 0,89 & 0,21 & 0,58 \\
\hline & & & nach Bef. & 18 & 1,47 & 1,01 & 0,24 & 0,66 \\
\hline & & 3,3Mg@50kPa & vor Bef. & 12 & 1,36 & 0,70 & 0,20 & 0,56 \\
\hline & & & nach Bef. & 12 & 1,95 & 0,91 & 0,26 & 0,73 \\
\hline & & 3,3Mg@160kPa & vor Bef. & 12 & 1,16 & 0,76 & 0,22 & 0,60 \\
\hline & & & nach Bef. & 12 & 1,95 & 0,68 & 0,20 & 0,54 \\
\hline & & 6,3Mg@160kPa & vor Bef. & 12 & 1,27 & 0,71 & 0,20 & 0,57 \\
\hline & & & nach Bef. & 12 & 1,94 & 0,76 & 0,22 & 0,61 \\
\hline & & 6,3Mg@250kPa & vor Bef. & 12 & 1,33 & 0,76 & 0,22 & 0,61 \\
\hline & & & nach Bef. & 12 & 1,98 & 0,36 & 0,10 & 0,29 \\
\hline & & 7,5Mg@250kPa & vor Bef. & 12 & 1,14 & 0,77 & 0,22 & 0,61 \\
\hline & & & nach Bef. & 12 & 2,11 & 0,40 & 0,12 & 0,32 \\
\hline & & 7,5Mg@350kPa & vor Bef. & 18 & 1,22 & 0,56 & 0,13 & 0,36 \\
\hline & & & nach Bef. & 18 & 2,34 & 0,62 & 0,15 & 0,40 \\
\hline & $30-39$ & Kontrolle & vor Bef. & 18 & 3,78 & 0,89 & 0,21 & 0,58 \\
\hline & & & nach Bef. & 18 & 3,65 & 0,84 & 0,20 & 0,55 \\
\hline & & 3,3Mg@50kPa & vor Bef. & 12 & 3,72 & 1,23 & 0,35 & 0,98 \\
\hline & & & nach Bef. & 12 & 3,73 & 0,98 & 0,28 & 0,79 \\
\hline & & 3,3Mg@160kPa & vor Bef. & 12 & 3,36 & 0,65 & 0,19 & 0,52 \\
\hline & & & nach Bef. & 12 & 3,29 & 0,77 & 0,22 & 0,61 \\
\hline & & 6,3Mg@160kPa & vor Bef. & 12 & 3,29 & 0,87 & 0,25 & 0,70 \\
\hline & & & nach Bef. & 12 & 3,66 & 0,87 & 0,25 & 0,69 \\
\hline & & 6,3Mg@250kPa & vor Bef. & 12 & 4,12 & 0,96 & 0,28 & 0,77 \\
\hline & & & nach Bef. & 12 & 3,91 & 0,76 & 0,22 & 0,61 \\
\hline & & 7,5Mg@250kPa & vor Bef. & 12 & 4,14 & 1,21 & 0,35 & 0,97 \\
\hline & & & nach Bef. & 12 & 3,69 & 0,90 & 0,26 & 0,72 \\
\hline & & 7,5Mg@350kPa & vor Bef. & 18 & 3,64 & 1,31 & 0,31 & 0,86 \\
\hline & & & nach Bef. & 18 & 3,04 & 0,72 & 0,17 & 0,47 \\
\hline
\end{tabular}




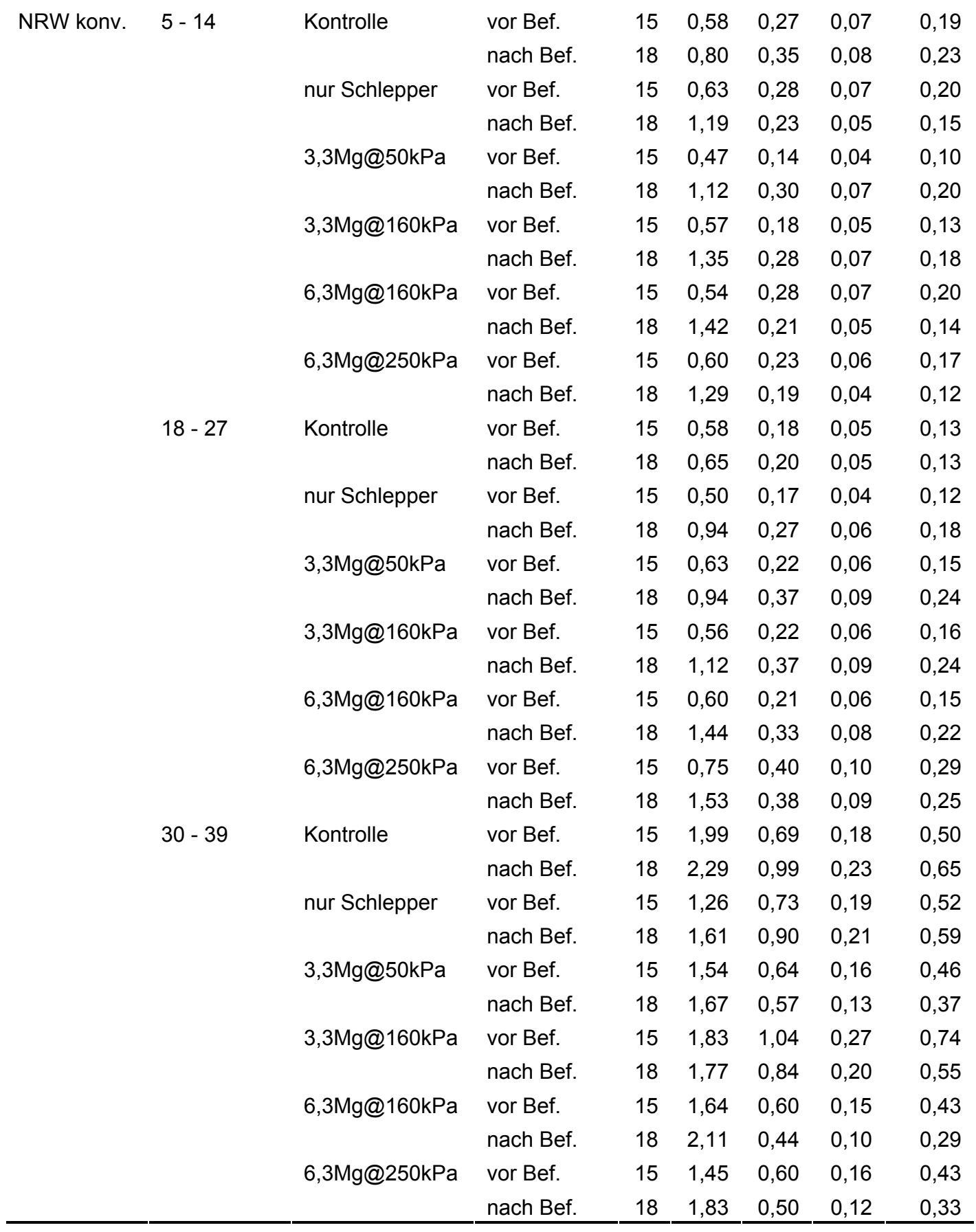


Tabelle A 17: Effekte eines Niederschlagsereignisses auf die vertikalen Eindringwiderstände vor und nach einer Bef. am Standort SH im Herbst 2006

\begin{tabular}{|c|c|c|c|c|c|c|c|c|}
\hline Fläche & MTB [cm] & Variante & Chronologie & $\mathrm{n}$ & M & SD & SED & $\mathrm{GD}_{5 \%}$ \\
\hline \multirow[t]{36}{*}{ SH kons. } & $5-14$ & Kontrolle & vor Bef. & 15 & 1,30 & 0,45 & 0,12 & 0,32 \\
\hline & & & nach Bef. & 18 & 1,00 & 0,30 & 0,07 & 0,20 \\
\hline & & 3,3Mg@50kPa & vor Bef. & 10 & 1,37 & 0,59 & 0,19 & 0,52 \\
\hline & & & nach Bef. & 12 & 0,76 & 0,32 & 0,09 & 0,25 \\
\hline & & 3,3Mg@160kPa & vor Bef. & 10 & 1,04 & 0,30 & 0,09 & 0,26 \\
\hline & & & nach Bef. & 12 & 0,55 & 0,09 & 0,03 & 0,07 \\
\hline & & 6,3Mg@160kPa & vor Bef. & 10 & 1,11 & 0,26 & 0,08 & 0,23 \\
\hline & & & nach Bef. & 12 & 0,70 & 0,12 & 0,03 & 0,10 \\
\hline & & 6,3Mg@250kPa & vor Bef. & 10 & 1,24 & 0,46 & 0,14 & 0,40 \\
\hline & & & nach Bef. & 12 & 0,65 & 0,18 & 0,05 & 0,14 \\
\hline & & 7,5Mg@250kPa & vor Bef. & 10 & 1,35 & 0,34 & 0,11 & 0,30 \\
\hline & & & nach Bef. & 12 & 0,67 & 0,23 & 0,07 & 0,18 \\
\hline & & 7,5Mg@350kPa & vor Bef. & 15 & 1,12 & 0,39 & 0,10 & 0,28 \\
\hline & & & nach Bef. & 18 & 0,71 & 0,18 & 0,04 & 0,11 \\
\hline & $18-27$ & Kontrolle & vor Bef. & 15 & 2,72 & 0,98 & 0,25 & 0,70 \\
\hline & & & nach Bef. & 18 & 1,94 & 0,58 & 0,14 & 0,38 \\
\hline & & 3,3Mg@50kPa & vor Bef. & 10 & 2,55 & 0,56 & 0,18 & 0,49 \\
\hline & & & nach Bef. & 12 & 1,68 & 0,19 & 0,06 & 0,16 \\
\hline & & 3,3Mg@160kPa & vor Bef. & 10 & 2,29 & 0,53 & 0,17 & 0,46 \\
\hline & & & nach Bef. & 12 & 1,39 & 0,28 & 0,08 & 0,23 \\
\hline & & 6,3Mg@160kPa & vor Bef. & 10 & 2,12 & 0,24 & 0,08 & 0,21 \\
\hline & & & nach Bef. & 12 & 1,39 & 0,13 & 0,04 & 0,10 \\
\hline & & 6,3Mg@250kPa & vor Bef. & 10 & 2,23 & 0,34 & 0,11 & 0,29 \\
\hline & & & nach Bef. & 12 & 1,62 & 0,32 & 0,09 & 0,26 \\
\hline & & 7,5Mg@250kPa & vor Bef. & 10 & 2,35 & 0,58 & 0,18 & 0,51 \\
\hline & & & nach Bef. & 12 & 1,54 & 0,34 & 0,10 & 0,27 \\
\hline & & 7,5Mg@350kPa & vor Bef. & 15 & 2,20 & 0,39 & 0,10 & 0,28 \\
\hline & & & nach Bef. & 18 & 1,85 & 0,55 & 0,13 & 0,36 \\
\hline & $30-39$ & Kontrolle & vor Bef. & 15 & 2,42 & 1,12 & 0,29 & 0,80 \\
\hline & & & nach Bef. & 18 & 2,35 & 0,91 & 0,21 & 0,59 \\
\hline & & 3,3Mg@50kPa & vor Bef. & 10 & 2,44 & 0,94 & 0,30 & 0,83 \\
\hline & & & nach Bef. & 12 & 2,24 & 0,80 & 0,23 & 0,64 \\
\hline & & 3,3Mg@160kPa & vor Bef. & 10 & 2,22 & 0,50 & 0,16 & 0,44 \\
\hline & & & nach Bef. & 12 & 1,91 & 0,65 & 0,19 & 0,52 \\
\hline & & 6,3Mg@160kPa & vor Bef. & 10 & 1,87 & 0,43 & 0,13 & 0,37 \\
\hline & & & nach Bef. & 12 & 1,48 & 0,35 & 0,10 & 0,28 \\
\hline
\end{tabular}




\begin{tabular}{|c|c|c|c|c|c|c|c|c|}
\hline & & 6,3Mg@250kPa & vor Bef. & 10 & 2,25 & 0,53 & 0,17 & 0,47 \\
\hline & & & nach Bef. & 12 & 2,18 & 0,54 & 0,16 & 0,43 \\
\hline & & 7,5Mg@250kPa & vor Bef. & 10 & 2,70 & 0,63 & 0,20 & 0,55 \\
\hline & & & nach Bef. & 12 & 2,00 & 0,43 & 0,12 & 0,34 \\
\hline & & 7,5Mg@350kPa & vor Bef. & 15 & 2,00 & 0,69 & 0,18 & 0,49 \\
\hline & & & nach Bef. & 18 & 1,89 & 0,54 & 0,13 & 0,35 \\
\hline & $50-59$ & Kontrolle & vor Bef. & 15 & 2,34 & 0,90 & 0,23 & 0,65 \\
\hline & & & nach Bef. & 18 & 2,03 & 0,79 & 0,19 & 0,51 \\
\hline & & 3,3Mg@50kPa & vor Bef. & 10 & 2,40 & 1,28 & 0,41 & 1,12 \\
\hline & & & nach Bef. & 12 & 2,06 & 0,55 & 0,16 & 0,44 \\
\hline & & 3,3Mg@160kPa & vor Bef. & 10 & 2,21 & 0,92 & 0,29 & 0,81 \\
\hline & & & nach Bef. & 12 & 1,68 & 0,58 & 0,17 & 0,46 \\
\hline & & 6,3Mg@160kPa & vor Bef. & 10 & 1,48 & 0,47 & 0,15 & 0,41 \\
\hline & & & nach Bef. & 12 & 1,20 & 0,13 & 0,04 & 0,10 \\
\hline & & 6,3Mg@250kPa & vor Bef. & 10 & 2,12 & 1,06 & 0,33 & 0,93 \\
\hline & & & nach Bef. & 12 & 1,97 & 0,62 & 0,18 & 0,50 \\
\hline & & 7,5Mg@250kPa & vor Bef. & 10 & 2,05 & 0,41 & 0,13 & 0,36 \\
\hline & & & nach Bef. & 12 & 1,78 & 0,43 & 0,12 & 0,34 \\
\hline & & 7,5Mg@350kPa & vor Bef. & 15 & 1,73 & 0,52 & 0,13 & 0,37 \\
\hline & & & nach Bef. & 18 & 1,85 & 0,60 & 0,14 & 0,39 \\
\hline H konv. & $5-14$ & Kontrolle & vor Bef. & 18 & 2,84 & 0,77 & 0,18 & 0,50 \\
\hline & & & nach Bef. & 15 & 2,68 & 0,76 & 0,20 & 0,55 \\
\hline & & 3,3Mg@50kPa & vor Bef. & 12 & 2,20 & 0,30 & 0,09 & 0,24 \\
\hline & & & nach Bef. & 10 & 2,71 & 0,55 & 0,17 & 0,48 \\
\hline & & 3,3Mg@160kPa & vor Bef. & 12 & 2,15 & 0,38 & 0,11 & 0,30 \\
\hline & & & nach Bef. & 10 & 2,22 & 0,89 & 0,28 & 0,78 \\
\hline & & 6,3Mg@160kPa & vor Bef. & 12 & 2,12 & 0,39 & 0,11 & 0,31 \\
\hline & & & nach Bef. & 10 & 2,27 & 0,52 & 0,17 & 0,46 \\
\hline & & 6,3Mg@250kPa & vor Bef. & 12 & 2,46 & 0,48 & 0,14 & 0,39 \\
\hline & & & nach Bef. & 10 & 2,72 & 0,60 & 0,19 & 0,52 \\
\hline & & 7,5Mg@250kPa & vor Bef. & 12 & 2,61 & 0,55 & 0,16 & 0,44 \\
\hline & & & nach Bef. & 10 & 2,30 & 0,61 & 0,19 & 0,54 \\
\hline & & 7,5Mg@350kPa & vor Bef. & 18 & 2,56 & 0,78 & 0,18 & 0,51 \\
\hline & & & nach Bef. & 15 & 2,22 & 0,61 & 0,16 & 0,44 \\
\hline & $18-27$ & Kontrolle & vor Bef. & 18 & 2,92 & 0,86 & 0,20 & 0,56 \\
\hline & & & nach Bef. & 15 & 3,34 & 0,76 & 0,20 & 0,54 \\
\hline & & 3,3Mg@50kPa & vor Bef. & 12 & 2,83 & 0,75 & 0,22 & 0,60 \\
\hline & & & nach Bef. & 10 & 2,99 & 0,88 & 0,28 & 0,77 \\
\hline & & 3,3Mg@160kPa & vor Bef. & 12 & 2,76 & 0,70 & 0,20 & 0,56 \\
\hline & & & nach Bef. & 10 & 2,60 & 0,84 & 0,27 & 0,74 \\
\hline
\end{tabular}




\begin{tabular}{|c|c|c|c|c|c|c|c|}
\hline & 6,3Mg@160kPa & vor Bef. & 12 & 2,45 & 0,48 & 0,14 & 0,38 \\
\hline & & nach Bef. & 10 & 2,62 & 1,06 & 0,33 & 0,93 \\
\hline & 6,3Mg@250kPa & vor Bef. & 12 & 2,81 & 0,85 & 0,25 & 0,68 \\
\hline & & nach Bef. & 10 & 2,79 & 0,74 & 0,23 & 0,65 \\
\hline & 7,5Mg@250kPa & vor Bef. & 12 & 3,90 & 0,98 & 0,28 & 0,78 \\
\hline & & nach Bef. & 10 & 2,38 & 0,72 & 0,23 & 0,63 \\
\hline & 7,5Mg@350kPa & vor Bef. & 18 & 3,47 & 0,89 & 0,21 & 0,58 \\
\hline & & nach Bef. & 15 & 2,23 & 1,12 & 0,29 & 0,80 \\
\hline $30-39$ & Kontrolle & vor Bef. & 18 & 4,44 & 0,93 & 0,22 & 0,61 \\
\hline & & nach Bef. & 15 & 4,01 & 1,33 & 0,34 & 0,95 \\
\hline & 3,3Mg@50kPa & vor Bef. & 12 & 4,30 & 1,10 & 0,32 & 0,88 \\
\hline & & nach Bef. & 10 & 4,41 & 1,31 & 0,41 & 1,15 \\
\hline & 3,3Mg@160kPa & vor Bef. & 12 & 3,49 & 0,80 & 0,23 & 0,64 \\
\hline & & nach Bef. & 10 & 3,20 & 1,07 & 0,34 & 0,94 \\
\hline & 6,3Mg@160kPa & vor Bef. & 12 & 3,99 & 1,12 & 0,32 & 0,89 \\
\hline & & nach Bef. & 10 & 4,03 & 0,81 & 0,26 & 0,71 \\
\hline & 6,3Mg@250kPa & vor Bef. & 12 & 4,74 & 0,80 & 0,23 & 0,64 \\
\hline & & nach Bef. & 10 & 4,03 & 1,13 & 0,36 & 0,99 \\
\hline & 7,5Mg@250kPa & vor Bef. & 12 & 5,56 & 0,80 & 0,23 & 0,64 \\
\hline & & nach Bef. & 10 & 4,01 & 1,08 & 0,34 & 0,95 \\
\hline & 7,5Mg@350kPa & vor Bef. & 18 & 4,44 & 1,52 & 0,36 & 0,99 \\
\hline & & nach Bef. & 15 & 3,66 & 1,42 & 0,37 & 1,01 \\
\hline $50-59$ & Kontrolle & vor Bef. & 18 & 3,95 & 1,43 & 0,34 & 0,93 \\
\hline & & nach Bef. & 15 & 2,96 & 0,90 & 0,23 & 0,64 \\
\hline & 3,3Mg@50kPa & vor Bef. & 12 & 4,92 & 1,49 & 0,43 & 1,19 \\
\hline & & nach Bef. & 10 & 3,30 & 1,26 & 0,40 & 1,11 \\
\hline & 3,3Mg@160kPa & vor Bef. & 12 & 3,33 & 1,13 & 0,33 & 0,91 \\
\hline & & nach Bef. & 10 & 3,08 & 1,12 & 0,35 & 0,98 \\
\hline & 6,3Mg@160kPa & vor Bef. & 12 & 3,69 & 1,32 & 0,38 & 1,06 \\
\hline & & nach Bef. & 10 & 3,24 & 1,40 & 0,44 & 1,22 \\
\hline & 6,3Mg@250kPa & vor Bef. & 12 & 4,32 & 1,52 & 0,44 & 1,22 \\
\hline & & nach Bef. & 10 & 3,54 & 1,32 & 0,42 & 1,15 \\
\hline & 7,5Mg@250kPa & vor Bef. & 11 & 4,20 & 1,37 & 0,41 & 1,15 \\
\hline & & nach Bef. & 10 & 3,42 & 1,15 & 0,36 & 1,01 \\
\hline & 7,5Mg@350kPa & vor Bef. & 18 & 4,07 & 1,26 & 0,30 & 0,82 \\
\hline & & nach Bef. & 15 & 3,64 & 1,07 & 0,28 & 0,77 \\
\hline
\end{tabular}


Tabelle A 18: Gravimetrische Bodenwassergehalte [\%] der Flächen $\mathrm{SH}_{\text {kons }}$ und $\mathrm{SH}_{\text {konv }}$ im Herbst 2006 zum Zeitpunkt der Vertikalpenetrometermessungen vor der Bef. (22./23.10.2006) und nach der Bef. (30./31.10.2006)

\begin{tabular}{lllllll}
\hline Fläche & Tiefe $[\mathrm{cm}]$ & Chronologie & $\mathrm{n}$ & $\mathrm{M}$ & $\mathrm{SD}$ & SED \\
\hline $\mathrm{SH}_{\text {kons }}$ & 0 & vor der Bef. & 20 & 20,4 & 2,3 & 0,5 \\
& & nach der Bef. & 20 & 21,9 & 1,6 & 0,4 \\
& \multirow{2}{*}{20} & vor der Bef. & 20 & 15,6 & 1,4 & 0,3 \\
& & nach der Bef. & 20 & 17,4 & 1,2 & 0,3 \\
& \multirow{2}{*}{40} & vor der Bef. & 20 & 17,2 & 1,4 & 0,3 \\
& & nach der Bef. & 20 & 17,4 & 0,5 & 0,1 \\
& \multirow{2}{*}{0} & vor der Bef. & 20 & 18,2 & 1,3 & 0,3 \\
$\mathrm{SH}_{\text {konv }}$ & nach der Bef. & 20 & 15,1 & 1,4 & 0,3 \\
& \multirow{2}{*}{20} & vor der Bef. & 20 & 15,9 & 1,5 & 0,3 \\
& nach der Bef. & 20 & 14,7 & 1,7 & 0,4 \\
& \multirow{2}{*}{40} & vor der Bef. & 20 & 15,2 & 2,8 & 0,6 \\
& nach der Bef. & 20 & 13,5 & 1,9 & 0,4 \\
\hline
\end{tabular}


Tabelle A 19: Vertikale Eindringwiderstände nach Fläche, kalendarischem Termin und Messtiefenbereich

\begin{tabular}{|c|c|c|c|c|c|c|c|}
\hline Fläche & MTB [cm] & Termin & $\mathrm{n}$ & $M$ & SD & SED & $\mathrm{GD}_{5 \%}$ \\
\hline \multirow[t]{16}{*}{$\mathrm{SH}_{\mathrm{kons}}$} & \multirow[t]{4}{*}{$5-14$} & Herbst 2006 & 176 & 0,96 & 0,41 & 0,03 & 0,09 \\
\hline & & Frühjahr 2007 & 186 & 2,93 & 0,96 & 0,07 & 0,20 \\
\hline & & Herbst 2007 & 192 & 0,95 & 0,30 & 0,02 & 0,06 \\
\hline & & Frühjahr 2008 & 192 & 1,05 & 0,30 & 0,02 & 0,06 \\
\hline & \multirow[t]{4}{*}{$18-27$} & Herbst 2006 & 176 & 1,98 & 0,63 & 0,05 & 0,13 \\
\hline & & Frühjahr 2007 & 183 & 3,77 & 0,95 & 0,07 & 0,19 \\
\hline & & Herbst 2007 & 192 & 2,09 & 0,41 & 0,03 & 0,08 \\
\hline & & Frühjahr 2008 & 192 & 1,94 & 0,44 & 0,03 & 0,09 \\
\hline & \multirow[t]{4}{*}{$30-39$} & Herbst 2006 & 176 & 2,13 & 0,73 & 0,06 & 0,15 \\
\hline & & Frühjahr 2007 & 183 & 3,10 & 0,89 & 0,07 & 0,18 \\
\hline & & Herbst 2007 & 192 & 2,28 & 0,73 & 0,05 & 0,15 \\
\hline & & Frühjahr 2008 & 192 & 2,19 & 0,60 & 0,04 & 0,12 \\
\hline & \multirow[t]{4}{*}{$50-59$} & Herbst 2006 & 176 & 1,92 & 0,75 & 0,06 & 0,16 \\
\hline & & Frühjahr 2007 & 180 & 2,45 & 0,96 & 0,07 & 0,20 \\
\hline & & Herbst 2007 & 181 & 1,82 & 0,72 & 0,05 & 0,15 \\
\hline & & Frühjahr 2008 & 179 & 1,74 & 0,67 & 0,05 & 0,14 \\
\hline \multirow[t]{16}{*}{$\mathrm{SH}_{\mathrm{konv}}$} & \multirow[t]{4}{*}{$5-14$} & Herbst 2006 & 176 & 2,45 & 0,65 & 0,05 & 0,14 \\
\hline & & Frühjahr 2007 & 192 & 1,36 & 0,49 & 0,04 & 0,10 \\
\hline & & Herbst 2007 & 192 & 1,64 & 0,46 & 0,03 & 0,09 \\
\hline & & Frühjahr 2008 & 192 & 1,43 & 0,46 & 0,03 & 0,09 \\
\hline & \multirow[t]{4}{*}{$18-27$} & Herbst 2006 & 176 & 2,89 & 0,93 & 0,07 & 0,19 \\
\hline & & Frühjahr 2007 & 192 & 1,61 & 0,81 & 0,06 & 0,16 \\
\hline & & Herbst 2007 & 191 & 2,16 & 0,69 & 0,05 & 0,14 \\
\hline & & Frühjahr 2008 & 192 & 1,89 & 0,64 & 0,05 & 0,13 \\
\hline & \multirow[t]{4}{*}{$30-39$} & Herbst 2006 & 176 & 4,19 & 1,22 & 0,09 & 0,25 \\
\hline & & Frühjahr 2007 & 192 & 3,63 & 0,96 & 0,07 & 0,19 \\
\hline & & Herbst 2007 & 190 & 3,55 & 0,94 & 0,07 & 0,19 \\
\hline & & Frühjahr 2008 & 192 & 3,29 & 0,89 & 0,06 & 0,18 \\
\hline & \multirow[t]{4}{*}{$50-59$} & Herbst 2006 & 175 & 3,72 & 1,33 & 0,10 & 0,28 \\
\hline & & Frühjahr 2007 & 190 & 2,88 & 1,32 & 0,10 & 0,27 \\
\hline & & Herbst 2007 & 160 & 2,83 & 1,27 & 0,10 & 0,28 \\
\hline & & Frühjahr 2008 & 181 & 2,33 & 1,01 & 0,08 & 0,21 \\
\hline \multirow[t]{4}{*}{$\mathrm{NRW}_{\text {kons }}$} & \multirow[t]{4}{*}{$5-14$} & Frühjahr 2006 & 156 & 1,20 & 0,33 & 0,03 & 0,07 \\
\hline & & Herbst 2006 & 180 & 1,75 & 0,50 & 0,04 & 0,10 \\
\hline & & Frühjahr 2007 & 216 & 1,59 & 0,61 & 0,04 & 0,11 \\
\hline & & Herbst 2007 & 216 & 1,31 & 0,33 & 0,02 & 0,06 \\
\hline
\end{tabular}




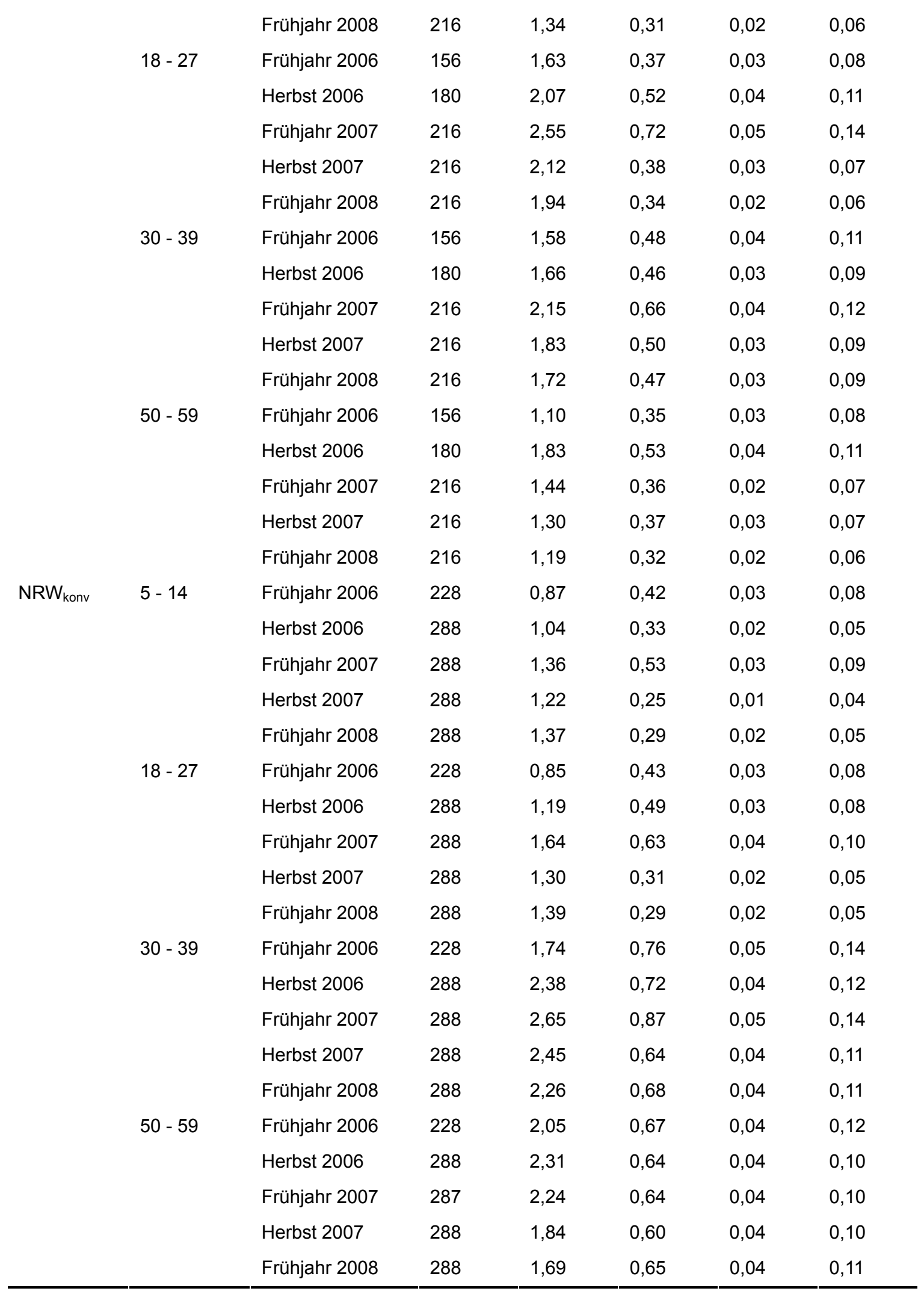


Tabelle A 20: Vertikaler Eindringwiderstand [MPa] bei Varianten unterschiedlicher Radlasten bei $160 \mathrm{kPa}$ Reifeninnendruck nach Fläche und Messtiefenbereich

\begin{tabular}{|c|c|c|c|c|c|c|c|}
\hline Fläche & MTB [cm] & $\mathrm{RL}[\mathrm{Mg}]$ & $n$ & $\mathrm{M}$ & SD & SED & $\mathrm{GD}_{5 \%}$ \\
\hline \multirow[t]{10}{*}{$\mathrm{SH}_{\text {kons }}$} & $5-14$ & 3,3 & 48 & 1,36 & 1,17 & 0,17 & 0,47 \\
\hline & & 6,3 & 48 & 1,37 & 1,02 & 0,15 & 0,41 \\
\hline & $18-27$ & 3,3 & 48 & 2,26 & 1,08 & 0,16 & 0,43 \\
\hline & & 6,3 & 48 & 2,24 & 0,91 & 0,13 & 0,37 \\
\hline & $30-39$ & 3,3 & 48 & 2,37 & 0,82 & 0,12 & 0,33 \\
\hline & & 6,3 & 48 & 2,05 & 0,89 & 0,13 & 0,36 \\
\hline & $50-59$ & 3,3 & 47 & 1,94 & 0,76 & 0,11 & 0,31 \\
\hline & & 6,3 & 48 & 1,59 & 0,71 & 0,10 & 0,29 \\
\hline & Insgesamt & 3,3 & 191 & 1,98 & 1,05 & 0,08 & 0,21 \\
\hline & & 6,3 & 192 & 1,82 & 0,95 & 0,07 & 0,19 \\
\hline \multirow[t]{10}{*}{$\mathrm{SH}_{\text {konv }}$} & $5-14$ & 3,3 & 46 & 1,67 & 0,62 & 0,09 & 0,25 \\
\hline & & 6,3 & 46 & 1,73 & 0,47 & 0,07 & 0,19 \\
\hline & $18-27$ & 3,3 & 45 & 2,03 & 0,67 & 0,10 & 0,28 \\
\hline & & 6,3 & 46 & 2,07 & 0,77 & 0,11 & 0,31 \\
\hline & $30-39$ & 3,3 & 45 & 3,03 & 0,91 & 0,14 & 0,38 \\
\hline & & 6,3 & 46 & 3,50 & 0,81 & 0,12 & 0,33 \\
\hline & $50-59$ & 3,3 & 44 & 2,47 & 1,01 & 0,15 & 0,42 \\
\hline & & 6,3 & 46 & 2,51 & 1,07 & 0,16 & 0,44 \\
\hline & Insgesamt & 3,3 & 180 & 2,30 & 0,96 & 0,07 & 0,20 \\
\hline & & 6,3 & 184 & 2,45 & 1,04 & 0,08 & 0,21 \\
\hline \multirow[t]{10}{*}{$\mathrm{NRW}_{\text {kons }}$} & $5-14$ & 3,3 & 60 & 1,44 & 0,53 & 0,07 & 0,19 \\
\hline & & 6,3 & 60 & 1,51 & 0,57 & 0,07 & 0,20 \\
\hline & $18-27$ & 3,3 & 60 & 2,25 & 0,52 & 0,07 & 0,19 \\
\hline & & 6,3 & 60 & 2,25 & 0,55 & 0,07 & 0,20 \\
\hline & $30-39$ & 3,3 & 60 & 2,02 & 0,61 & 0,08 & 0,22 \\
\hline & & 6,3 & 60 & 1,97 & 0,54 & 0,07 & 0,19 \\
\hline & $50-59$ & 3,3 & 60 & 1,34 & 0,41 & 0,05 & 0,15 \\
\hline & & 6,3 & 60 & 1,38 & 0,46 & 0,06 & 0,16 \\
\hline & Insgesamt & 3,3 & 240 & 1,76 & 0,65 & 0,04 & 0,12 \\
\hline & & 6,3 & 240 & 1,78 & 0,63 & 0,04 & 0,11 \\
\hline \multirow[t]{6}{*}{$\mathrm{NRW}_{\text {konv }}$} & $5-14$ & 3,3 & 90 & 1,33 & 0,32 & 0,03 & 0,09 \\
\hline & & 6,3 & 90 & 1,47 & 0,35 & 0,04 & 0,10 \\
\hline & $18-27$ & 3,3 & 90 & 1,39 & 0,46 & 0,05 & 0,14 \\
\hline & & 6,3 & 90 & 1,64 & 0,52 & 0,05 & 0,15 \\
\hline & $30-39$ & 3,3 & 90 & 2,20 & 0,67 & 0,07 & 0,19 \\
\hline & & 6,3 & 90 & 2,62 & 0,84 & 0,09 & 0,25 \\
\hline
\end{tabular}




\begin{tabular}{|c|c|c|c|c|c|c|c|}
\hline & $50-59$ & 3,3 & 90 & 2,10 & 0,68 & 0,07 & 0,20 \\
\hline & & 6,3 & 90 & 2,10 & 0,85 & 0,09 & 0,25 \\
\hline & Insgesamt & 3,3 & 360 & 1,75 & 0,68 & 0,04 & 0,10 \\
\hline & & 6,3 & 360 & 1,96 & 0,81 & 0,04 & 0,12 \\
\hline Insgesamt & $5-14$ & 3,3 & 244 & 1,43 & 0,68 & 0,04 & 0,12 \\
\hline & & 6,3 & 244 & 1,51 & 0,62 & 0,04 & 0,11 \\
\hline & $18-27$ & 3,3 & 243 & 1,89 & 0,78 & 0,05 & 0,14 \\
\hline & & 6,3 & 244 & 1,99 & 0,72 & 0,05 & 0,13 \\
\hline & $30-39$ & 3,3 & 243 & 2,34 & 0,81 & 0,05 & 0,14 \\
\hline & & 6,3 & 244 & 2,51 & 0,95 & 0,06 & 0,17 \\
\hline & $50-59$ & 3,3 & 241 & 1,95 & 0,81 & 0,05 & 0,14 \\
\hline & & 6,3 & 244 & 1,90 & 0,89 & 0,06 & 0,16 \\
\hline & Insgesamt & 3,3 & 971 & 1,90 & 0,84 & 0,03 & 0,07 \\
\hline & & 6,3 & 976 & 1,98 & 0,88 & 0,03 & 0,08 \\
\hline
\end{tabular}

Tabelle A 21: Vertikaler Eindringwiderstand [MPa] bei Varianten unterschiedlicher Radlasten bei 250 kPa Reifeninnendruck nach Fläche und Messtiefenbereich

\begin{tabular}{|c|c|c|c|c|c|c|c|}
\hline Fläche & MTB [cm] & $\mathrm{RL}[\mathrm{Mg}]$ & $\mathrm{n}$ & $M$ & SD & SED & $\mathrm{GD}_{5 \%}$ \\
\hline \multirow[t]{10}{*}{$\mathrm{SH}_{\text {kons }}$} & \multirow[t]{2}{*}{$5-14$} & 6,3 & 48 & 1,26 & 0,95 & 0,14 & 0,38 \\
\hline & & 7,5 & 48 & 1,29 & 0,88 & 0,13 & 0,35 \\
\hline & \multirow[t]{2}{*}{$18-27$} & 6,3 & 48 & 2,23 & 1,12 & 0,16 & 0,45 \\
\hline & & 7,5 & 48 & 2,34 & 1,00 & 0,14 & 0,40 \\
\hline & \multirow[t]{2}{*}{$30-39$} & 6,3 & 48 & 2,35 & 0,89 & 0,13 & 0,36 \\
\hline & & 7,5 & 48 & 2,33 & 0,59 & 0,08 & 0,23 \\
\hline & \multirow[t]{2}{*}{$50-59$} & 6,3 & 48 & 2,03 & 0,79 & 0,11 & 0,31 \\
\hline & & 7,5 & 47 & 1,93 & 0,66 & 0,10 & 0,27 \\
\hline & \multirow[t]{2}{*}{ Insgesamt } & 6,3 & 192 & 1,97 & 1,03 & 0,07 & 0,21 \\
\hline & & 7,5 & 191 & 1,97 & 0,90 & 0,07 & 0,18 \\
\hline \multirow[t]{10}{*}{$\mathrm{SH}_{\text {konv }}$} & \multirow[t]{2}{*}{$5-14$} & 6,3 & 46 & 1,91 & 0,65 & 0,10 & 0,27 \\
\hline & & 7,5 & 46 & 1,86 & 0,52 & 0,08 & 0,21 \\
\hline & \multirow[t]{2}{*}{$18-27$} & 6,3 & 46 & 2,23 & 0,71 & 0,11 & 0,29 \\
\hline & & 7,5 & 46 & 2,19 & 0,64 & 0,09 & 0,26 \\
\hline & \multirow[t]{2}{*}{$30-39$} & 6,3 & 46 & 3,59 & 0,91 & 0,13 & 0,37 \\
\hline & & 7,5 & 46 & 3,56 & 0,81 & 0,12 & 0,33 \\
\hline & \multirow[t]{2}{*}{$50-59$} & 6,3 & 45 & 3,15 & 1,49 & 0,22 & 0,61 \\
\hline & & 7,5 & 42 & 3,41 & 1,37 & 0,21 & 0,59 \\
\hline & \multirow[t]{2}{*}{ Insgesamt } & 6,3 & 183 & 2,72 & 1,20 & 0,09 & 0,25 \\
\hline & & 7,5 & 180 & 2,74 & 1,15 & 0,09 & 0,24 \\
\hline
\end{tabular}




\begin{tabular}{|c|c|c|c|c|c|c|c|}
\hline \multirow[t]{10}{*}{$N_{R} W_{\text {kons }}$} & $5-14$ & 6,3 & 48 & 1,48 & 0,43 & 0,06 & 0,17 \\
\hline & & 7,5 & 48 & 1,63 & 0,55 & 0,08 & 0,22 \\
\hline & $18-27$ & 6,3 & 48 & 2,29 & 0,52 & 0,07 & 0,21 \\
\hline & & 7,5 & 48 & 2,24 & 0,42 & 0,06 & 0,17 \\
\hline & $30-39$ & 6,3 & 48 & 2,00 & 0,57 & 0,08 & 0,23 \\
\hline & & 7,5 & 48 & 1,82 & 0,52 & 0,07 & 0,21 \\
\hline & $50-59$ & 6,3 & 48 & 1,26 & 0,33 & 0,05 & 0,13 \\
\hline & & 7,5 & 48 & 1,44 & 0,48 & 0,07 & 0,19 \\
\hline & Insgesamt & 6,3 & 192 & 1,76 & 0,62 & 0,04 & 0,12 \\
\hline & & 7,5 & 192 & 1,78 & 0,57 & 0,04 & 0,11 \\
\hline \multirow[t]{10}{*}{$\mathrm{NRW}_{\text {konv }}$} & $5-14$ & 6,3 & 72 & 1,48 & 0,43 & 0,05 & 0,14 \\
\hline & & 7,5 & 72 & 1,42 & 0,34 & 0,04 & 0,11 \\
\hline & $18-27$ & 6,3 & 72 & 1,68 & 0,45 & 0,05 & 0,15 \\
\hline & & 7,5 & 72 & 1,63 & 0,40 & 0,05 & 0,13 \\
\hline & $30-39$ & 6,3 & 72 & 2,61 & 0,75 & 0,09 & 0,25 \\
\hline & & 7,5 & 72 & 2,45 & 0,63 & 0,07 & 0,21 \\
\hline & $50-59$ & 6,3 & 72 & 2,04 & 0,55 & 0,06 & 0,18 \\
\hline & & 7,5 & 72 & 2,03 & 0,73 & 0,09 & 0,24 \\
\hline & Insgesamt & 6,3 & 288 & 1,95 & 0,70 & 0,04 & 0,11 \\
\hline & & 7,5 & 288 & 1,88 & 0,67 & 0,04 & 0,11 \\
\hline \multirow[t]{10}{*}{ Insgesamt } & $5-14$ & 6,3 & 214 & 1,52 & 0,66 & 0,05 & 0,13 \\
\hline & & 7,5 & 214 & 1,53 & 0,61 & 0,04 & 0,12 \\
\hline & $18-27$ & 6,3 & 214 & 2,06 & 0,76 & 0,05 & 0,14 \\
\hline & & 7,5 & 214 & 2,05 & 0,70 & 0,05 & 0,13 \\
\hline & $30-39$ & 6,3 & 214 & 2,62 & 0,96 & 0,07 & 0,18 \\
\hline & & 7,5 & 214 & 2,52 & 0,87 & 0,06 & 0,17 \\
\hline & $50-59$ & 6,3 & 213 & 2,10 & 1,06 & 0,07 & 0,20 \\
\hline & & 7,5 & 209 & 2,15 & 1,07 & 0,07 & 0,21 \\
\hline & Insgesamt & 6,3 & 855 & 2,08 & 0,95 & 0,03 & 0,09 \\
\hline & & 7,5 & 851 & 2,06 & 0,90 & 0,03 & 0,09 \\
\hline
\end{tabular}


Tabelle A 22: Vertikaler Eindringwiderstand [MPa] bei Varianten mit unterschiedlichem Reifeninnendruck (RID) bei 3,3 Mg Radlast nach Fläche und Messtiefenbereich (MTB)

\begin{tabular}{|c|c|c|c|c|c|c|c|}
\hline Fläche & MTB [cm] & $\mathrm{RID}[\mathrm{kPa}]$ & $\mathrm{n}$ & $M$ & SD & SED & $\mathrm{GD}_{5 \%}$ \\
\hline \multirow[t]{10}{*}{$\mathrm{SH}_{\text {kons }}$} & \multirow[t]{2}{*}{$5-14$} & 50 & 48 & 1,22 & 0,81 & 0,12 & 0,32 \\
\hline & & 160 & 48 & 1,36 & 1,17 & 0,17 & 0,47 \\
\hline & \multirow[t]{2}{*}{$18-27$} & 50 & 48 & 2,28 & 1,01 & 0,15 & 0,40 \\
\hline & & 160 & 48 & 2,26 & 1,08 & 0,16 & 0,43 \\
\hline & \multirow[t]{2}{*}{$30-39$} & 50 & 48 & 2,45 & 0,92 & 0,13 & 0,37 \\
\hline & & 160 & 48 & 2,37 & 0,82 & 0,12 & 0,33 \\
\hline & \multirow[t]{2}{*}{$50-59$} & 50 & 45 & 2,12 & 1,08 & 0,16 & 0,45 \\
\hline & & 160 & 47 & 1,94 & 0,76 & 0,11 & 0,31 \\
\hline & \multirow[t]{2}{*}{ Insgesamt } & 50 & 189 & 2,02 & 1,06 & 0,08 & 0,21 \\
\hline & & 160 & 191 & 1,98 & 1,05 & 0,08 & 0,21 \\
\hline \multirow[t]{10}{*}{$\mathrm{SH}_{\mathrm{konv}}$} & \multirow[t]{2}{*}{$5-14$} & 50 & 46 & 1,81 & 0,63 & 0,09 & 0,26 \\
\hline & & 160 & 46 & 1,67 & 0,62 & 0,09 & 0,25 \\
\hline & \multirow[t]{2}{*}{$18-27$} & 50 & 46 & 2,11 & 0,84 & 0,12 & 0,34 \\
\hline & & 160 & 45 & 2,03 & 0,67 & 0,10 & 0,28 \\
\hline & \multirow[t]{2}{*}{$30-39$} & 50 & 46 & 3,91 & 1,04 & 0,15 & 0,42 \\
\hline & & 160 & 45 & 3,03 & 0,91 & 0,14 & 0,38 \\
\hline & \multirow[t]{2}{*}{$50-59$} & 50 & 42 & 2,93 & 1,34 & 0,21 & 0,58 \\
\hline & & 160 & 44 & 2,47 & 1,01 & 0,15 & 0,42 \\
\hline & \multirow[t]{2}{*}{ Insgesamt } & 50 & 180 & 2,68 & 1,28 & 0,10 & 0,26 \\
\hline & & 160 & 180 & 2,30 & 0,96 & 0,07 & 0,20 \\
\hline \multirow[t]{10}{*}{$\mathrm{NRW}_{\text {kons }}$} & \multirow[t]{2}{*}{$5-14$} & 50 & 60 & 1,47 & 0,42 & 0,05 & 0,15 \\
\hline & & 160 & 60 & 1,44 & 0,53 & 0,07 & 0,19 \\
\hline & \multirow[t]{2}{*}{$18-27$} & 50 & 60 & 2,20 & 0,64 & 0,08 & 0,23 \\
\hline & & 160 & 60 & 2,25 & 0,52 & 0,07 & 0,19 \\
\hline & \multirow[t]{2}{*}{$30-39$} & 50 & 60 & 2,02 & 0,55 & 0,07 & 0,20 \\
\hline & & 160 & 60 & 2,02 & 0,61 & 0,08 & 0,22 \\
\hline & \multirow[t]{2}{*}{$50-59$} & 50 & 60 & 1,28 & 0,43 & 0,06 & 0,15 \\
\hline & & 160 & 60 & 1,34 & 0,41 & 0,05 & 0,15 \\
\hline & \multirow[t]{2}{*}{ Insgesamt } & 50 & 240 & 1,74 & 0,64 & 0,04 & 0,11 \\
\hline & & 160 & 240 & 1,76 & 0,65 & 0,04 & 0,12 \\
\hline \multirow[t]{6}{*}{$\mathrm{NRW}_{\text {konv }}$} & \multirow[t]{2}{*}{$5-14$} & 50 & 90 & 1,33 & 0,32 & 0,03 & 0,09 \\
\hline & & 160 & 90 & 1,33 & 0,32 & 0,03 & 0,09 \\
\hline & \multirow[t]{2}{*}{$18-27$} & 50 & 90 & 1,23 & 0,38 & 0,04 & 0,11 \\
\hline & & 160 & 90 & 1,39 & 0,46 & 0,05 & 0,14 \\
\hline & \multirow[t]{2}{*}{$30-39$} & 50 & 90 & 2,06 & 0,68 & 0,07 & 0,20 \\
\hline & & 160 & 90 & 2,20 & 0,67 & 0,07 & 0,19 \\
\hline
\end{tabular}




\begin{tabular}{|c|c|c|c|c|c|c|c|}
\hline & $50-59$ & 50 & 90 & 2,18 & 0,86 & 0,09 & 0,25 \\
\hline & & 160 & 90 & 2,10 & 0,68 & 0,07 & 0,20 \\
\hline & Insgesamt & 50 & 360 & 1,70 & 0,73 & 0,04 & 0,11 \\
\hline & & 160 & 360 & 1,75 & 0,68 & 0,04 & 0,10 \\
\hline Insgesamt & $5-14$ & 50 & 244 & 1,43 & 0,56 & 0,04 & 0,10 \\
\hline & & 160 & 244 & 1,43 & 0,68 & 0,04 & 0,12 \\
\hline & $18-27$ & 50 & 244 & 1,84 & 0,84 & 0,05 & 0,15 \\
\hline & & 160 & 243 & 1,89 & 0,78 & 0,05 & 0,14 \\
\hline & $30-39$ & 50 & 244 & 2,47 & 1,06 & 0,07 & 0,19 \\
\hline & & 160 & 243 & 2,34 & 0,81 & 0,05 & 0,14 \\
\hline & $50-59$ & 50 & 237 & 2,08 & 1,07 & 0,07 & 0,19 \\
\hline & & 160 & 241 & 1,95 & 0,81 & 0,05 & 0,14 \\
\hline & Insgesamt & 50 & 969 & 1,96 & 0,98 & 0,03 & 0,09 \\
\hline & & 160 & 971 & 1,90 & 0,84 & 0,03 & 0,07 \\
\hline
\end{tabular}

Tabelle A 23: Vertikaler Eindringwiderstand [MPa] bei Varianten mit unterschiedlichem Reifeninnendruck (RID) bei 6,3 Mg Radlast nach Fläche und Messtiefenbereich (MTB)

\begin{tabular}{|c|c|c|c|c|c|c|c|}
\hline Fläche & MTB [cm] & $\mathrm{RID}[\mathrm{kPa}]$ & $\mathrm{n}$ & $M$ & SD & SED & $\mathrm{GD}_{5 \%}$ \\
\hline \multirow[t]{10}{*}{$\mathrm{SH}_{\text {kons }}$} & \multirow[t]{2}{*}{$5-14$} & 160 & 48 & 1,37 & 1,02 & 0,15 & 0,41 \\
\hline & & 250 & 48 & 1,26 & 0,95 & 0,14 & 0,38 \\
\hline & \multirow[t]{2}{*}{$18-27$} & 160 & 48 & 2,24 & 0,91 & 0,13 & 0,37 \\
\hline & & 250 & 48 & 2,23 & 1,12 & 0,16 & 0,45 \\
\hline & \multirow[t]{2}{*}{$30-39$} & 160 & 48 & 2,05 & 0,89 & 0,13 & 0,36 \\
\hline & & 250 & 48 & 2,35 & 0,89 & 0,13 & 0,36 \\
\hline & \multirow[t]{2}{*}{$50-59$} & 160 & 48 & 1,59 & 0,71 & 0,10 & 0,29 \\
\hline & & 250 & 48 & 2,03 & 0,79 & 0,11 & 0,31 \\
\hline & \multirow[t]{2}{*}{ Insgesamt } & 160 & 192 & 1,82 & 0,95 & 0,07 & 0,19 \\
\hline & & 250 & 192 & 1,97 & 1,03 & 0,07 & 0,21 \\
\hline \multirow[t]{10}{*}{$\mathrm{SH}_{\text {konv }}$} & \multirow[t]{2}{*}{$5-14$} & 160 & 46 & 1,73 & 0,47 & 0,07 & 0,19 \\
\hline & & 250 & 46 & 1,91 & 0,65 & 0,10 & 0,27 \\
\hline & \multirow[t]{2}{*}{$18-27$} & 160 & 46 & 2,07 & 0,77 & 0,11 & 0,31 \\
\hline & & 250 & 46 & 2,23 & 0,71 & 0,11 & 0,29 \\
\hline & \multirow[t]{2}{*}{$30-39$} & 160 & 46 & 3,50 & 0,81 & 0,12 & 0,33 \\
\hline & & 250 & 46 & 3,59 & 0,91 & 0,13 & 0,37 \\
\hline & \multirow[t]{2}{*}{$50-59$} & 160 & 46 & 2,51 & 1,07 & 0,16 & 0,44 \\
\hline & & 250 & 45 & 3,15 & 1,49 & 0,22 & 0,61 \\
\hline & \multirow[t]{2}{*}{ Insgesamt } & 160 & 184 & 2,45 & 1,04 & 0,08 & 0,21 \\
\hline & & 250 & 183 & 2,72 & 1,20 & 0,09 & 0,25 \\
\hline
\end{tabular}




\begin{tabular}{|c|c|c|c|c|c|c|c|}
\hline \multirow[t]{10}{*}{$N_{R} W_{\text {kons }}$} & $5-14$ & 160 & 60 & 1,51 & 0,57 & 0,07 & 0,20 \\
\hline & & 250 & 60 & 1,36 & 0,45 & 0,06 & 0,16 \\
\hline & $18-27$ & 160 & 60 & 2,25 & 0,55 & 0,07 & 0,20 \\
\hline & & 250 & 60 & 2,08 & 0,63 & 0,08 & 0,23 \\
\hline & $30-39$ & 160 & 60 & 1,97 & 0,54 & 0,07 & 0,19 \\
\hline & & 250 & 60 & 1,84 & 0,61 & 0,08 & 0,22 \\
\hline & $50-59$ & 160 & 60 & 1,38 & 0,46 & 0,06 & 0,16 \\
\hline & & 250 & 60 & 1,22 & 0,34 & 0,04 & 0,12 \\
\hline & Insgesamt & 160 & 240 & 1,78 & 0,63 & 0,04 & 0,11 \\
\hline & & 250 & 240 & 1,63 & 0,63 & 0,04 & 0,11 \\
\hline \multirow[t]{10}{*}{$\mathrm{NRW}_{\text {konv }}$} & $5-14$ & 160 & 90 & 1,47 & 0,35 & 0,04 & 0,10 \\
\hline & & 250 & 90 & 1,44 & 0,40 & 0,04 & 0,12 \\
\hline & $18-27$ & 160 & 90 & 1,64 & 0,52 & 0,05 & 0,15 \\
\hline & & 250 & 90 & 1,65 & 0,44 & 0,05 & 0,13 \\
\hline & $30-39$ & 160 & 90 & 2,62 & 0,84 & 0,09 & 0,25 \\
\hline & & 250 & 90 & 2,45 & 0,77 & 0,08 & 0,23 \\
\hline & $50-59$ & 160 & 90 & 2,10 & 0,85 & 0,09 & 0,25 \\
\hline & & 250 & 90 & 2,07 & 0,52 & 0,06 & 0,15 \\
\hline & Insgesamt & 160 & 360 & 1,96 & 0,81 & 0,04 & 0,12 \\
\hline & & 250 & 360 & 1,90 & 0,67 & 0,04 & 0,10 \\
\hline \multirow[t]{10}{*}{ Insgesamt } & $5-14$ & 160 & 244 & 1,51 & 0,62 & 0,04 & 0,11 \\
\hline & & 250 & 244 & 1,48 & 0,64 & 0,04 & 0,11 \\
\hline & $18-27$ & 160 & 244 & 1,99 & 0,72 & 0,05 & 0,13 \\
\hline & & 250 & 244 & 1,98 & 0,75 & 0,05 & 0,13 \\
\hline & $30-39$ & 160 & 244 & 2,51 & 0,95 & 0,06 & 0,17 \\
\hline & & 250 & 244 & 2,50 & 0,98 & 0,06 & 0,17 \\
\hline & $50-59$ & 160 & 244 & 1,90 & 0,89 & 0,06 & 0,16 \\
\hline & & 250 & 243 & 2,05 & 1,02 & 0,07 & 0,18 \\
\hline & Insgesamt & 160 & 976 & 1,98 & 0,88 & 0,03 & 0,08 \\
\hline & & 250 & 975 & 2,00 & 0,93 & 0,03 & 0,08 \\
\hline
\end{tabular}


Tabelle A 24: Vertikaler Eindringwiderstand [MPa] bei Varianten mit unterschiedlichem Reifeninnendruck bei 7,5 Mg Radlast nach Fläche und Messtiefenbereich (ohne Block 3)

\begin{tabular}{|c|c|c|c|c|c|c|c|}
\hline Fläche & MTB [cm] & $\mathrm{RID}[\mathrm{kPa}]$ & $\mathrm{n}$ & $M$ & SD & SED & $\mathrm{GD}_{5 \%}$ \\
\hline \multirow[t]{10}{*}{$\mathrm{SH}_{\text {kons }}$} & \multirow[t]{2}{*}{$5-14$} & 250 & 48 & 1,29 & 0,88 & 0,13 & 0,35 \\
\hline & & 350 & 48 & 1,41 & 0,97 & 0,14 & 0,39 \\
\hline & \multirow[t]{2}{*}{$18-27$} & 250 & 48 & 2,34 & 1,00 & 0,14 & 0,40 \\
\hline & & 350 & 48 & 2,37 & 1,12 & 0,16 & 0,45 \\
\hline & \multirow[t]{2}{*}{$30-39$} & 250 & 48 & 2,33 & 0,59 & 0,08 & 0,23 \\
\hline & & 350 & 48 & 2,23 & 0,75 & 0,11 & 0,30 \\
\hline & \multirow[t]{2}{*}{$50-59$} & 250 & 47 & 1,93 & 0,66 & 0,10 & 0,27 \\
\hline & & 350 & 48 & 1,63 & 0,45 & 0,07 & 0,18 \\
\hline & \multirow[t]{2}{*}{ Insgesamt } & 250 & 191 & 1,97 & 0,90 & 0,07 & 0,18 \\
\hline & & 350 & 192 & 1,91 & 0,95 & 0,07 & 0,19 \\
\hline \multirow[t]{10}{*}{$\mathrm{SH}_{\mathrm{konv}}$} & \multirow[t]{2}{*}{$5-14$} & 250 & 46 & 1,86 & 0,52 & 0,08 & 0,21 \\
\hline & & 350 & 46 & 1,72 & 0,47 & 0,07 & 0,19 \\
\hline & \multirow[t]{2}{*}{$18-27$} & 250 & 46 & 2,19 & 0,64 & 0,09 & 0,26 \\
\hline & & 350 & 46 & 2,22 & 0,67 & 0,10 & 0,27 \\
\hline & \multirow[t]{2}{*}{$30-39$} & 250 & 46 & 3,56 & 0,81 & 0,12 & 0,33 \\
\hline & & 350 & 46 & 3,30 & 1,06 & 0,16 & 0,43 \\
\hline & \multirow[t]{2}{*}{$50-59$} & 250 & 42 & 3,41 & 1,37 & 0,21 & 0,59 \\
\hline & & 350 & 45 & 2,72 & 1,10 & 0,16 & 0,45 \\
\hline & \multirow[t]{2}{*}{ Insgesamt } & 250 & 180 & 2,74 & 1,15 & 0,09 & 0,24 \\
\hline & & 350 & 183 & 2,49 & 1,04 & 0,08 & 0,21 \\
\hline \multirow[t]{10}{*}{$\mathrm{NRW}_{\text {kons }}$} & \multirow[t]{2}{*}{$5-14$} & 250 & 48 & 1,63 & 0,55 & 0,08 & 0,22 \\
\hline & & 350 & 48 & 1,61 & 0,58 & 0,08 & 0,23 \\
\hline & \multirow[t]{2}{*}{$18-27$} & 250 & 48 & 2,24 & 0,42 & 0,06 & 0,17 \\
\hline & & 350 & 48 & 2,23 & 0,57 & 0,08 & 0,23 \\
\hline & \multirow[t]{2}{*}{$30-39$} & 250 & 48 & 1,82 & 0,52 & 0,07 & 0,21 \\
\hline & & 350 & 48 & 1,87 & 0,54 & 0,08 & 0,22 \\
\hline & \multirow[t]{2}{*}{$50-59$} & 250 & 48 & 1,44 & 0,48 & 0,07 & 0,19 \\
\hline & & 350 & 48 & 1,44 & 0,56 & 0,08 & 0,23 \\
\hline & \multirow[t]{2}{*}{ Insgesamt } & 250 & 192 & 1,78 & 0,57 & 0,04 & 0,11 \\
\hline & & 350 & 192 & 1,79 & 0,63 & 0,05 & 0,13 \\
\hline \multirow[t]{6}{*}{$\mathrm{NRW}_{\text {konv }}$} & \multirow[t]{2}{*}{$5-14$} & 250 & 48 & 1,39 & 0,34 & 0,05 & 0,14 \\
\hline & & 350 & 48 & 1,41 & 0,49 & 0,07 & 0,20 \\
\hline & \multirow[t]{2}{*}{$18-27$} & 250 & 48 & 1,64 & 0,44 & 0,06 & 0,18 \\
\hline & & 350 & 48 & 1,56 & 0,46 & 0,07 & 0,18 \\
\hline & \multirow[t]{2}{*}{$30-39$} & 250 & 48 & 2,25 & 0,50 & 0,07 & 0,20 \\
\hline & & 350 & 48 & 2,33 & 0,58 & 0,08 & 0,23 \\
\hline
\end{tabular}




\begin{tabular}{|c|c|c|c|c|c|c|c|}
\hline & $50-59$ & 250 & 48 & 1,74 & 0,49 & 0,07 & 0,20 \\
\hline & & 350 & 48 & 1,89 & 0,51 & 0,07 & 0,21 \\
\hline & Insgesamt & 250 & 192 & 1,76 & 0,55 & 0,04 & 0,11 \\
\hline & & 350 & 192 & 1,80 & 0,62 & 0,04 & 0,12 \\
\hline \multirow[t]{10}{*}{ Insgesamt } & $5-14$ & 250 & 190 & 1,54 & 0,64 & 0,05 & 0,13 \\
\hline & & 350 & 190 & 1,53 & 0,67 & 0,05 & 0,13 \\
\hline & $18-27$ & 250 & 190 & 2,10 & 0,72 & 0,05 & 0,14 \\
\hline & & 350 & 190 & 2,10 & 0,81 & 0,06 & 0,16 \\
\hline & $30-39$ & 250 & 190 & 2,48 & 0,89 & 0,06 & 0,18 \\
\hline & & 350 & 190 & 2,42 & 0,92 & 0,07 & 0,18 \\
\hline & $50-59$ & 250 & 185 & 2,09 & 1,09 & 0,08 & 0,22 \\
\hline & & 350 & 189 & 1,91 & 0,84 & 0,06 & 0,17 \\
\hline & Insgesamt & 250 & 755 & 2,05 & 0,91 & 0,03 & 0,09 \\
\hline & & 350 & 759 & 1,99 & 0,87 & 0,03 & 0,09 \\
\hline
\end{tabular}

Tabelle A 25: Horizontale Eindringwiderstände [MPa] nach Jahr und Messtiefenbereich gemittelt über Varianten und Flächen

\begin{tabular}{lllllll}
\hline Jahr & MTB [cm] & $\mathrm{n}$ & $\mathrm{M}$ & $\mathrm{SD}$ & SED & GD $_{5 \%}$ \\
\hline 2006 & $5-14$ & 438 & 0,65 & 0,20 & 0,01 & 0,03 \\
& $18-27$ & 438 & 0,86 & 0,22 & 0,01 & 0,03 \\
& $30-39$ & 438 & 1,25 & 0,48 & 0,02 & 0,06 \\
2007 & $5-14$ & 444 & 0,67 & 0,16 & 0,01 & 0,02 \\
& $18-27$ & 444 & 0,90 & 0,21 & 0,01 & 0,03 \\
& $30-39$ & 348 & 1,14 & 0,23 & 0,01 & 0,03 \\
2008 & $5-14$ & 435 & 0,60 & 0,13 & 0,01 & 0,02 \\
& $18-27$ & 435 & 0,72 & 0,14 & 0,01 & 0,02 \\
& $30-39$ & 435 & 0,99 & 0,33 & 0,02 & 0,04 \\
\hline
\end{tabular}


Tabelle A 26: Horizontale Eindringwiderstände [MPa] der Fläche $\mathrm{SH}_{\text {kons }}$ im Jahr 2006 nach Messtiefenbereich und Belastungsvariante

\begin{tabular}{|c|c|c|c|c|c|c|}
\hline MTB [cm] & Variante & $\mathrm{n}$ & M & SD & SED & $\mathrm{GD}_{5 \%}$ \\
\hline \multirow[t]{7}{*}{$5-14$} & Kontrolle & 15 & 0,38 & 0,08 & 0,02 & 0,06 \\
\hline & 3,3Mg@50kPa & 12 & 0,39 & 0,04 & 0,01 & 0,03 \\
\hline & 3,3Mg@160kPa & 12 & 0,38 & 0,06 & 0,02 & 0,05 \\
\hline & 6,3Mg@160kPa & 10 & 0,45 & 0,05 & 0,02 & 0,04 \\
\hline & 6,3Mg@250kPa & 12 & 0,41 & 0,07 & 0,02 & 0,06 \\
\hline & 7,5Mg@250kPa & 12 & 0,36 & 0,04 & 0,01 & 0,03 \\
\hline & 7,5Mg@350kPa & 18 & 0,39 & 0,06 & 0,01 & 0,04 \\
\hline \multirow[t]{7}{*}{$18-27$} & Kontrolle & 15 & 0,79 & 0,16 & 0,04 & 0,11 \\
\hline & 3,3Mg@50kPa & 12 & 0,80 & 0,05 & 0,01 & 0,04 \\
\hline & 3,3Mg@160kPa & 12 & 0,73 & 0,08 & 0,02 & 0,07 \\
\hline & 6,3Mg@160kPa & 10 & 0,91 & 0,08 & 0,03 & 0,07 \\
\hline & 6,3Mg@250kPa & 12 & 0,79 & 0,09 & 0,03 & 0,08 \\
\hline & 7,5Mg@250kPa & 12 & 0,82 & 0,09 & 0,03 & 0,07 \\
\hline & 7,5Mg@350kPa & 18 & 0,86 & 0,12 & 0,03 & 0,08 \\
\hline \multirow[t]{7}{*}{$30-39$} & Kontrolle & 15 & 1,37 & 0,21 & 0,05 & 0,15 \\
\hline & 3,3Mg@50kPa & 12 & 1,34 & 0,18 & 0,05 & 0,14 \\
\hline & 3,3Mg@160kPa & 12 & 1,27 & 0,16 & 0,05 & 0,13 \\
\hline & 6,3Mg@160kPa & 10 & 1,35 & 0,24 & 0,08 & 0,21 \\
\hline & 6,3Mg@250kPa & 12 & 1,29 & 0,13 & 0,04 & 0,10 \\
\hline & 7,5Mg@250kPa & 12 & 1,32 & 0,12 & 0,03 & 0,09 \\
\hline & 7,5Mg@350kPa & 18 & 1,35 & 0,12 & 0,03 & 0,08 \\
\hline
\end{tabular}


Tabelle A 27: Horizontale Eindringwiderstände [MPa] der Fläche $\mathrm{SH}_{\text {kons }}$ im Jahr 2007 nach Messtiefenbereich und Belastungsvariante

\begin{tabular}{|c|c|c|c|c|c|c|}
\hline MTB [cm] & Variante & $\mathrm{n}$ & M & SD & SED & $\mathrm{GD}_{5 \%}$ \\
\hline \multirow[t]{7}{*}{$5-14$} & Kontrolle & 18 & 0,56 & 0,10 & 0,02 & 0,07 \\
\hline & 3,3Mg@50kPa & 12 & 0,60 & 0,13 & 0,04 & 0,10 \\
\hline & 3,3Mg@160kPa & 12 & 0,63 & 0,18 & 0,05 & 0,14 \\
\hline & 6,3Mg@160kPa & 12 & 0,60 & 0,12 & 0,03 & 0,09 \\
\hline & 6,3Mg@250kPa & 12 & 0,51 & 0,15 & 0,04 & 0,12 \\
\hline & 7,5Mg@250kPa & 12 & 0,56 & 0,21 & 0,06 & 0,17 \\
\hline & 7,5Mg@350kPa & 18 & 0,52 & 0,09 & 0,02 & 0,06 \\
\hline \multirow[t]{7}{*}{$18-27$} & Kontrolle & 18 & 0,99 & 0,15 & 0,04 & 0,10 \\
\hline & 3,3Mg@50kPa & 12 & 0,98 & 0,12 & 0,04 & 0,10 \\
\hline & 3,3Mg@160kPa & 12 & 1,04 & 0,10 & 0,03 & 0,08 \\
\hline & 6,3Mg@160kPa & 12 & 1,02 & 0,09 & 0,03 & 0,07 \\
\hline & 6,3Mg@250kPa & 12 & 1,00 & 0,16 & 0,05 & 0,12 \\
\hline & 7,5Mg@250kPa & 12 & 1,05 & 0,20 & 0,06 & 0,16 \\
\hline & 7,5Mg@350kPa & 18 & 0,95 & 0,12 & 0,03 & 0,08 \\
\hline \multirow[t]{7}{*}{$30-39$} & Kontrolle & 18 & 1,31 & 0,18 & 0,04 & 0,12 \\
\hline & 3,3Mg@50kPa & 12 & 1,36 & 0,18 & 0,05 & 0,14 \\
\hline & 3,3Mg@160kPa & 12 & 1,31 & 0,16 & 0,05 & 0,13 \\
\hline & 6,3Mg@160kPa & 12 & 1,37 & 0,15 & 0,04 & 0,12 \\
\hline & 6,3Mg@250kPa & 12 & 1,48 & 0,29 & 0,09 & 0,24 \\
\hline & 7,5Mg@250kPa & 12 & 1,45 & 0,26 & 0,08 & 0,21 \\
\hline & 7,5Mg@350kPa & 18 & 1,28 & 0,26 & 0,06 & 0,17 \\
\hline
\end{tabular}


Tabelle A 28: Horizontale Eindringwiderstände [MPa] der Fläche $\mathrm{SH}_{\text {kons }}$ im Jahr 2008 nach Messtiefenbereich und Belastungsvariante

\begin{tabular}{|c|c|c|c|c|c|c|}
\hline MTB [cm] & Variante & $\mathrm{n}$ & $\mathrm{M}$ & SD & SED & $\mathrm{GD}_{5 \%}$ \\
\hline \multirow[t]{7}{*}{$5-14$} & Kontrolle & 9 & 0,51 & 0,03 & 0,01 & 0,02 \\
\hline & 3,3Mg@50kPa & 12 & 0,59 & 0,12 & 0,04 & 0,10 \\
\hline & 3,3Mg@160kPa & 12 & 0,52 & 0,12 & 0,04 & 0,10 \\
\hline & 6,3Mg@160kPa & 12 & 0,50 & 0,19 & 0,06 & 0,15 \\
\hline & 6,3Mg@250kPa & 12 & 0,53 & 0,16 & 0,05 & 0,13 \\
\hline & 7,5Mg@250kPa & 12 & 0,59 & 0,05 & 0,02 & 0,04 \\
\hline & 7,5Mg@350kPa & 18 & 0,60 & 0,10 & 0,02 & 0,07 \\
\hline \multirow[t]{7}{*}{$18-27$} & Kontrolle & 9 & 0,76 & 0,11 & 0,04 & 0,10 \\
\hline & 3,3Mg@50kPa & 12 & 0,92 & 0,12 & 0,04 & 0,10 \\
\hline & 3,3Mg@160kPa & 12 & 0,81 & 0,11 & 0,03 & 0,09 \\
\hline & 6,3Mg@160kPa & 12 & 0,82 & 0,08 & 0,02 & 0,07 \\
\hline & 6,3Mg@250kPa & 12 & 0,84 & 0,11 & 0,03 & 0,08 \\
\hline & 7,5Mg@250kPa & 12 & 0,93 & 0,09 & 0,03 & 0,07 \\
\hline & 7,5Mg@350kPa & 18 & 0,97 & 0,13 & 0,03 & 0,09 \\
\hline \multirow[t]{7}{*}{$30-39$} & Kontrolle & 9 & 0,93 & 0,23 & 0,08 & 0,22 \\
\hline & 3,3Mg@50kPa & 12 & 1,17 & 0,23 & 0,07 & 0,18 \\
\hline & 3,3Mg@160kPa & 12 & 1,10 & 0,31 & 0,09 & 0,25 \\
\hline & 6,3Mg@160kPa & 12 & 1,15 & 0,13 & 0,04 & 0,10 \\
\hline & 6,3Mg@250kPa & 12 & 1,05 & 0,15 & 0,04 & 0,12 \\
\hline & 7,5Mg@250kPa & 12 & 1,18 & 0,13 & 0,04 & 0,10 \\
\hline & 7,5Mg@350kPa & 18 & 1,13 & 0,16 & 0,04 & 0,10 \\
\hline
\end{tabular}


Tabelle A 29: Horizontale Eindringwiderstände [MPa] der Fläche $\mathrm{SH}_{\text {konv }}$ im Jahr 2006 nach Messtiefenbereich und Belastungsvariante

\begin{tabular}{|c|c|c|c|c|c|c|}
\hline MTB [cm] & Variante & $\mathrm{n}$ & $\mathrm{M}$ & SD & SED & $\mathrm{GD}_{5 \%}$ \\
\hline \multirow[t]{7}{*}{$5-14$} & Kontrolle & 18 & 0,82 & 0,28 & 0,07 & 0,19 \\
\hline & 3,3Mg@50kPa & 12 & 0,74 & 0,17 & 0,05 & 0,14 \\
\hline & 3,3Mg@160kPa & 11 & 0,85 & 0,13 & 0,04 & 0,11 \\
\hline & 6,3Mg@160kPa & 12 & 0,81 & 0,18 & 0,05 & 0,14 \\
\hline & 6,3Mg@250kPa & 12 & 0,73 & 0,22 & 0,06 & 0,18 \\
\hline & 7,5Mg@250kPa & 12 & 0,84 & 0,14 & 0,04 & 0,12 \\
\hline & 7,5Mg@350kPa & 18 & 0,96 & 0,22 & 0,05 & 0,15 \\
\hline \multirow[t]{7}{*}{$18-27$} & Kontrolle & 18 & 1,09 & 0,21 & 0,05 & 0,14 \\
\hline & 3,3Mg@50kPa & 12 & 0,82 & 0,10 & 0,03 & 0,08 \\
\hline & 3,3Mg@160kPa & 11 & 0,91 & 0,17 & 0,05 & 0,14 \\
\hline & 6,3Mg@160kPa & 12 & 0,88 & 0,12 & 0,04 & 0,10 \\
\hline & 6,3Mg@250kPa & 12 & 0,89 & 0,13 & 0,04 & 0,11 \\
\hline & 7,5Mg@250kPa & 12 & 0,90 & 0,17 & 0,05 & 0,14 \\
\hline & 7,5Mg@350kPa & 18 & 0,97 & 0,20 & 0,05 & 0,13 \\
\hline \multirow[t]{7}{*}{$30-39$} & Kontrolle & 18 & 1,91 & 0,39 & 0,09 & 0,25 \\
\hline & 3,3Mg@50kPa & 12 & 1,91 & 0,38 & 0,11 & 0,30 \\
\hline & 3,3Mg@160kPa & 11 & 1,85 & 0,31 & 0,09 & 0,26 \\
\hline & 6,3Mg@160kPa & 12 & 1,74 & 0,46 & 0,13 & 0,37 \\
\hline & 6,3Mg@250kPa & 12 & 1,98 & 0,29 & 0,08 & 0,24 \\
\hline & 7,5Mg@250kPa & 12 & 2,02 & 0,87 & 0,25 & 0,70 \\
\hline & 7,5Mg@350kPa & 18 & 1,99 & 0,33 & 0,08 & 0,22 \\
\hline
\end{tabular}


Tabelle A 30: Horizontale Eindringwiderstände [MPa] der Fläche $\mathrm{SH}_{\text {konv }}$ im Jahr 2007 nach Messtiefenbereich und Belastungsvariante

\begin{tabular}{lllllll}
\hline MTB [cm] & Variante & $\mathrm{n}$ & $\mathrm{M}$ & $\mathrm{SD}$ & $\mathrm{SED}$ & $\mathrm{GD}_{5 \%}$ \\
\hline $5-14$ & Kontrolle & 18 & 0,71 & 0,18 & 0,04 & 0,12 \\
& $3,3 \mathrm{Mg} @ 50 \mathrm{kPa}$ & 12 & 0,84 & 0,19 & 0,06 & 0,16 \\
& $3,3 \mathrm{Mg} @ 160 \mathrm{kPa}$ & 12 & 0,82 & 0,14 & 0,04 & 0,12 \\
& $6,3 \mathrm{Mg} @ 160 \mathrm{kPa}$ & 12 & 0,75 & 0,10 & 0,03 & 0,08 \\
& $6,3 \mathrm{Mg} @ 250 \mathrm{kPa}$ & 12 & 0,85 & 0,17 & 0,05 & 0,14 \\
& $7,5 \mathrm{Mg} @ 250 \mathrm{kPa}$ & 12 & 0,78 & 0,22 & 0,06 & 0,18 \\
& $7,5 \mathrm{Mg} @ 350 \mathrm{kPa}$ & 18 & 0,72 & 0,25 & 0,06 & 0,17 \\
& Kontrolle 27 & 18 & 0,99 & 0,17 & 0,04 & 0,11 \\
& $3,3 \mathrm{Mg} @ 50 \mathrm{kPa}$ & 12 & 0,94 & 0,14 & 0,04 & 0,12 \\
& $3,3 \mathrm{Mg} @ 160 \mathrm{kPa}$ & 12 & 0,93 & 0,14 & 0,04 & 0,12 \\
& $6,3 \mathrm{Mg} @ 160 \mathrm{kPa}$ & 12 & 1,12 & 0,20 & 0,06 & 0,16 \\
& $6,3 \mathrm{Mg} @ 250 \mathrm{kPa}$ & 12 & 1,07 & 0,13 & 0,04 & 0,11 \\
& $7,5 \mathrm{Mg} @ 250 \mathrm{kPa}$ & 12 & 1,05 & 0,11 & 0,03 & 0,09 \\
& $7,5 \mathrm{Mg} @ 350 \mathrm{kPa}$ & 18 & 1,05 & 0,14 & 0,03 & 0,09 \\
\hline
\end{tabular}


Tabelle A 31: Horizontale Eindringwiderstände [MPa] der Fläche $\mathrm{SH}_{\text {konv }}$ im Jahr 2008 nach Messtiefenbereich und Belastungsvariante

\begin{tabular}{|c|c|c|c|c|c|c|}
\hline MTB [cm] & Variante & $\mathrm{n}$ & $\mathrm{M}$ & SD & SED & $\mathrm{GD}_{5 \%}$ \\
\hline \multirow[t]{7}{*}{$5-14$} & Kontrolle & 18 & 0,46 & 0,12 & 0,03 & 0,08 \\
\hline & 3,3Mg@50kPa & 12 & 0,51 & 0,13 & 0,04 & 0,11 \\
\hline & 3,3Mg@160kPa & 12 & 0,53 & 0,12 & 0,03 & 0,09 \\
\hline & 6,3Mg@160kPa & 12 & 0,49 & 0,13 & 0,04 & 0,10 \\
\hline & 6,3Mg@250kPa & 12 & 0,53 & 0,10 & 0,03 & 0,08 \\
\hline & 7,5Mg@250kPa & 12 & 0,50 & 0,12 & 0,04 & 0,10 \\
\hline & 7,5Mg@350kPa & 18 & 0,53 & 0,16 & 0,04 & 0,10 \\
\hline \multirow[t]{7}{*}{$18-27$} & Kontrolle & 18 & 0,62 & 0,16 & 0,04 & 0,11 \\
\hline & 3,3Mg@50kPa & 12 & 0,64 & 0,09 & 0,02 & 0,07 \\
\hline & 3,3Mg@160kPa & 12 & 0,62 & 0,11 & 0,03 & 0,09 \\
\hline & 6,3Mg@160kPa & 12 & 0,69 & 0,19 & 0,06 & 0,16 \\
\hline & 6,3Mg@250kPa & 12 & 0,71 & 0,09 & 0,02 & 0,07 \\
\hline & 7,5Mg@250kPa & 12 & 0,65 & 0,07 & 0,02 & 0,06 \\
\hline & 7,5Mg@350kPa & 18 & 0,78 & 0,13 & 0,03 & 0,09 \\
\hline \multirow[t]{7}{*}{$30-39$} & Kontrolle & 18 & 1,39 & 0,17 & 0,04 & 0,11 \\
\hline & 3,3Mg@50kPa & 12 & 1,50 & 0,46 & 0,13 & 0,37 \\
\hline & 3,3Mg@160kPa & 12 & 1,28 & 0,28 & 0,08 & 0,22 \\
\hline & 6,3Mg@160kPa & 12 & 1,26 & 0,16 & 0,05 & 0,13 \\
\hline & 6,3Mg@250kPa & 12 & 1,28 & 0,15 & 0,04 & 0,12 \\
\hline & 7,5Mg@250kPa & 12 & 1,28 & 0,25 & 0,07 & 0,20 \\
\hline & 7,5Mg@350kPa & 18 & 1,49 & 0,57 & 0,13 & 0,37 \\
\hline
\end{tabular}


Tabelle A 32: Horizontale Eindringwiderstände [MPa] der Fläche NRW kons im Jahr 2006 nach Messtiefenbereich und Belastungsvariante

\begin{tabular}{|c|c|c|c|c|c|c|}
\hline MTB [cm] & Variante & $\mathrm{n}$ & $\mathrm{M}$ & SD & SED & $\mathrm{GD}_{5 \%}$ \\
\hline \multirow[t]{7}{*}{$5-14$} & Kontrolle & 24 & 0,75 & 0,11 & 0,02 & 0,06 \\
\hline & 3,3Mg@50kPa & 12 & 0,76 & 0,11 & 0,03 & 0,09 \\
\hline & 3,3Mg@160kPa & 12 & 0,74 & 0,07 & 0,02 & 0,05 \\
\hline & 6,3Mg@160kPa & 12 & 0,81 & 0,07 & 0,02 & 0,06 \\
\hline & 6,3Mg@250kPa & 12 & 0,78 & 0,12 & 0,03 & 0,10 \\
\hline & 7,5Mg@250kPa & 12 & 0,75 & 0,06 & 0,02 & 0,05 \\
\hline & 7,5Mg@350kPa & 24 & 0,76 & 0,15 & 0,03 & 0,08 \\
\hline \multirow[t]{7}{*}{$18-27$} & Kontrolle & 24 & 1,15 & 0,13 & 0,03 & 0,08 \\
\hline & 3,3Mg@50kPa & 12 & 1,13 & 0,14 & 0,04 & 0,11 \\
\hline & 3,3Mg@160kPa & 12 & 1,05 & 0,14 & 0,04 & 0,11 \\
\hline & 6,3Mg@160kPa & 12 & 1,12 & 0,12 & 0,03 & 0,10 \\
\hline & 6,3Mg@250kPa & 12 & 1,08 & 0,12 & 0,03 & 0,10 \\
\hline & 7,5Mg@250kPa & 12 & 1,11 & 0,10 & 0,03 & 0,08 \\
\hline & 7,5Mg@350kPa & 24 & 1,14 & 0,11 & 0,02 & 0,06 \\
\hline \multirow[t]{7}{*}{$30-39$} & Kontrolle & 24 & 1,02 & 0,18 & 0,04 & 0,10 \\
\hline & 3,3Mg@50kPa & 12 & 0,93 & 0,09 & 0,03 & 0,07 \\
\hline & 3,3Mg@160kPa & 12 & 0,87 & 0,10 & 0,03 & 0,08 \\
\hline & 6,3Mg@160kPa & 12 & 0,90 & 0,11 & 0,03 & 0,09 \\
\hline & 6,3Mg@250kPa & 12 & 0,87 & 0,11 & 0,03 & 0,09 \\
\hline & 7,5Mg@250kPa & 12 & 0,90 & 0,19 & 0,06 & 0,15 \\
\hline & 7,5Mg@350kPa & 24 & 0,95 & 0,14 & 0,03 & 0,08 \\
\hline
\end{tabular}


Tabelle A 33: Horizontale Eindringwiderstände [MPa] der Fläche NRW $_{\text {kons }}$ im Jahr 2007 nach Messtiefenbereich und Belastungsvariante

\begin{tabular}{|c|c|c|c|c|c|c|}
\hline MTB [cm] & Variante & $\mathrm{n}$ & $\mathrm{M}$ & SD & SED & $\mathrm{GD}_{5 \%}$ \\
\hline \multirow[t]{7}{*}{$5-14$} & Kontrolle & 24 & 0,71 & 0,13 & 0,03 & 0,08 \\
\hline & 3,3Mg@50kPa & 12 & 0,78 & 0,08 & 0,02 & 0,07 \\
\hline & 3,3Mg@160kPa & 12 & 0,80 & 0,08 & 0,02 & 0,06 \\
\hline & 6,3Mg@160kPa & 12 & 0,82 & 0,09 & 0,03 & 0,07 \\
\hline & 6,3Mg@250kPa & 12 & 0,78 & 0,15 & 0,04 & 0,12 \\
\hline & 7,5Mg@250kPa & 12 & 0,77 & 0,12 & 0,04 & 0,10 \\
\hline & 7,5Mg@350kPa & 24 & 0,78 & 0,10 & 0,02 & 0,06 \\
\hline \multirow[t]{7}{*}{$18-27$} & Kontrolle & 24 & 1,04 & 0,14 & 0,03 & 0,08 \\
\hline & 3,3Mg@50kPa & 12 & 1,00 & 0,11 & 0,03 & 0,09 \\
\hline & 3,3Mg@160kPa & 12 & 1,02 & 0,10 & 0,03 & 0,08 \\
\hline & 6,3Mg@160kPa & 12 & 1,08 & 0,08 & 0,02 & 0,06 \\
\hline & 6,3Mg@250kPa & 12 & 1,02 & 0,14 & 0,04 & 0,11 \\
\hline & 7,5Mg@250kPa & 12 & 1,08 & 0,07 & 0,02 & 0,06 \\
\hline & 7,5Mg@350kPa & 24 & 1,05 & 0,13 & 0,03 & 0,08 \\
\hline \multirow[t]{7}{*}{$30-39$} & Kontrolle & 24 & 1,04 & 0,16 & 0,03 & 0,09 \\
\hline & 3,3Mg@50kPa & 12 & 0,99 & 0,13 & 0,04 & 0,11 \\
\hline & 3,3Mg@160kPa & 12 & 0,99 & 0,14 & 0,04 & 0,11 \\
\hline & 6,3Mg@160kPa & 12 & 0,98 & 0,15 & 0,04 & 0,12 \\
\hline & 6,3Mg@250kPa & 12 & 1,06 & 0,17 & 0,05 & 0,14 \\
\hline & 7,5Mg@250kPa & 12 & 1,01 & 0,15 & 0,04 & 0,12 \\
\hline & 7,5Mg@350kPa & 24 & 1,05 & 0,19 & 0,04 & 0,11 \\
\hline
\end{tabular}


Tabelle A 34: Horizontale Eindringwiderstände [MPa] der Fläche NRW $_{\text {kons }}$ im Jahr 2008 nach Messtiefenbereich und Belastungsvariante

\begin{tabular}{|c|c|c|c|c|c|c|}
\hline MTB [cm] & Variante & $\mathrm{n}$ & M & SD & SED & $\mathrm{GD}_{5 \%}$ \\
\hline \multirow[t]{7}{*}{$5-14$} & Kontrolle & 24 & 0,70 & 0,10 & 0,02 & 0,06 \\
\hline & 3,3Mg@50kPa & 12 & 0,73 & 0,07 & 0,02 & 0,06 \\
\hline & 3,3Mg@160kPa & 12 & 0,75 & 0,07 & 0,02 & 0,05 \\
\hline & 6,3Mg@160kPa & 12 & 0,71 & 0,05 & 0,01 & 0,04 \\
\hline & 6,3Mg@250kPa & 12 & 0,68 & 0,07 & 0,02 & 0,06 \\
\hline & 7,5Mg@250kPa & 12 & 0,77 & 0,06 & 0,02 & 0,05 \\
\hline & 7,5Mg@350kPa & 24 & 0,71 & 0,08 & 0,02 & 0,04 \\
\hline \multirow[t]{7}{*}{$18-27$} & Kontrolle & 24 & 0,74 & 0,06 & 0,01 & 0,03 \\
\hline & 3,3Mg@50kPa & 12 & 0,80 & 0,07 & 0,02 & 0,06 \\
\hline & 3,3Mg@160kPa & 12 & 0,71 & 0,06 & 0,02 & 0,05 \\
\hline & 6,3Mg@160kPa & 12 & 0,68 & 0,05 & 0,01 & 0,04 \\
\hline & 6,3Mg@250kPa & 12 & 0,83 & 0,07 & 0,02 & 0,06 \\
\hline & 7,5Mg@250kPa & 12 & 0,77 & 0,06 & 0,02 & 0,05 \\
\hline & 7,5Mg@350kPa & 24 & 0,81 & 0,10 & 0,02 & 0,06 \\
\hline \multirow[t]{7}{*}{$30-39$} & Kontrolle & 24 & 0,72 & 0,14 & 0,03 & 0,08 \\
\hline & 3,3Mg@50kPa & 12 & 0,66 & 0,06 & 0,02 & 0,05 \\
\hline & 3,3Mg@160kPa & 12 & 0,64 & 0,08 & 0,02 & 0,06 \\
\hline & 6,3Mg@160kPa & 12 & 0,63 & 0,06 & 0,02 & 0,05 \\
\hline & 6,3Mg@250kPa & 12 & 0,63 & 0,11 & 0,03 & 0,09 \\
\hline & 7,5Mg@250kPa & 12 & 0,67 & 0,04 & 0,01 & 0,03 \\
\hline & 7,5Mg@350kPa & 24 & 0,62 & 0,13 & 0,03 & 0,08 \\
\hline
\end{tabular}


Tabelle A 35: Horizontale Eindringwiderstände [MPa] der Fläche NRW konv im Jahr 2006 nach Messtiefenbereich und Belastungsvariante

\begin{tabular}{|c|c|c|c|c|c|c|}
\hline MTB [cm] & Variante & $\mathrm{n}$ & M & SD & SED & $\mathrm{GD}_{5 \%}$ \\
\hline \multirow[t]{8}{*}{$5-14$} & Kontrolle & 18 & 0,61 & 0,05 & 0,01 & 0,04 \\
\hline & nur Schlepper & 18 & 0,60 & 0,05 & 0,01 & 0,03 \\
\hline & 3,3Mg@50kPa & 18 & 0,62 & 0,06 & 0,01 & 0,04 \\
\hline & 3,3Mg@160kPa & 18 & 0,61 & 0,04 & 0,01 & 0,03 \\
\hline & 6,3Mg@160kPa & 18 & 0,63 & 0,03 & 0,01 & 0,02 \\
\hline & 6,3Mg@250kPa & 18 & 0,61 & 0,05 & 0,01 & 0,04 \\
\hline & 7,5Mg@250kPa & 18 & 0,60 & 0,06 & 0,01 & 0,04 \\
\hline & 7,5Mg@350kPa & 18 & 0,58 & 0,08 & 0,02 & 0,05 \\
\hline \multirow[t]{8}{*}{$18-27$} & Kontrolle & 18 & 0,57 & 0,07 & 0,02 & 0,05 \\
\hline & nur Schlepper & 18 & 0,60 & 0,08 & 0,02 & 0,06 \\
\hline & 3,3Mg@50kPa & 18 & 0,59 & 0,06 & 0,01 & 0,04 \\
\hline & 3,3Mg@160kPa & 18 & 0,65 & 0,06 & 0,02 & 0,04 \\
\hline & 6,3Mg@160kPa & 18 & 0,68 & 0,06 & 0,01 & 0,04 \\
\hline & 6,3Mg@250kPa & 18 & 0,67 & 0,09 & 0,02 & 0,06 \\
\hline & 7,5Mg@250kPa & 18 & 0,64 & 0,10 & 0,02 & 0,06 \\
\hline & 7,5Mg@350kPa & 18 & 0,71 & 0,08 & 0,02 & 0,05 \\
\hline \multirow[t]{8}{*}{$30-39$} & Kontrolle & 18 & 0,93 & 0,37 & 0,09 & 0,24 \\
\hline & nur Schlepper & 18 & 0,95 & 0,38 & 0,09 & 0,25 \\
\hline & 3,3Mg@50kPa & 18 & 0,89 & 0,21 & 0,05 & 0,14 \\
\hline & 3,3Mg@160kPa & 18 & 0,93 & 0,27 & 0,06 & 0,18 \\
\hline & 6,3Mg@160kPa & 18 & 1,05 & 0,26 & 0,06 & 0,17 \\
\hline & 6,3Mg@250kPa & 18 & 1,01 & 0,34 & 0,08 & 0,22 \\
\hline & 7,5Mg@250kPa & 18 & 1,07 & 0,23 & 0,05 & 0,15 \\
\hline & 7,5Mg@350kPa & 18 & 1,05 & 0,29 & 0,07 & 0,19 \\
\hline
\end{tabular}


Tabelle A 36: Horizontale Eindringwiderstände [MPa] der Fläche NRW konv im Jahr 2007 nach Messtiefenbereich und Belastungsvariante

\begin{tabular}{|c|c|c|c|c|c|c|}
\hline MTB [cm] & Variante & $\mathrm{n}$ & $M$ & SD & SED & $\mathrm{GD}_{5 \%}$ \\
\hline \multirow[t]{8}{*}{$5-14$} & Kontrolle & 18 & 0,58 & 0,07 & 0,02 & 0,04 \\
\hline & nur Schlepper & 18 & 0,62 & 0,05 & 0,01 & 0,03 \\
\hline & 3,3Mg@50kPa & 18 & 0,63 & 0,07 & 0,02 & 0,05 \\
\hline & 3,3Mg@160kPa & 18 & 0,60 & 0,06 & 0,01 & 0,04 \\
\hline & 6,3Mg@160kPa & 18 & 0,56 & 0,05 & 0,01 & 0,04 \\
\hline & 6,3Mg@250kPa & 18 & 0,60 & 0,11 & 0,03 & 0,07 \\
\hline & 7,5Mg@250kPa & 18 & 0,56 & 0,08 & 0,02 & 0,05 \\
\hline & 7,5Mg@350kPa & 18 & 0,61 & 0,11 & 0,03 & 0,07 \\
\hline \multirow[t]{8}{*}{$18-27$} & Kontrolle & 18 & 0,62 & 0,05 & 0,01 & 0,03 \\
\hline & nur Schlepper & 18 & 0,62 & 0,06 & 0,02 & 0,04 \\
\hline & 3,3Mg@50kPa & 18 & 0,67 & 0,05 & 0,01 & 0,03 \\
\hline & 3,3Mg@160kPa & 18 & 0,64 & 0,04 & 0,01 & 0,02 \\
\hline & 6,3Mg@160kPa & 18 & 0,66 & 0,06 & 0,01 & 0,04 \\
\hline & 6,3Mg@250kPa & 18 & 0,69 & 0,05 & 0,01 & 0,03 \\
\hline & 7,5Mg@250kPa & 18 & 0,71 & 0,06 & 0,01 & 0,04 \\
\hline & 7,5Mg@350kPa & 18 & 0,69 & 0,12 & 0,03 & 0,08 \\
\hline \multirow[t]{8}{*}{$30-39$} & Kontrolle & 18 & 0,98 & 0,14 & 0,03 & 0,09 \\
\hline & nur Schlepper & 18 & 0,99 & 0,15 & 0,03 & 0,10 \\
\hline & 3,3Mg@50kPa & 18 & 1,15 & 0,16 & 0,04 & 0,10 \\
\hline & 3,3Mg@160kPa & 18 & 1,05 & 0,16 & 0,04 & 0,11 \\
\hline & 6,3Mg@160kPa & 18 & 1,18 & 0,17 & 0,04 & 0,11 \\
\hline & 6,3Mg@250kPa & 18 & 1,09 & 0,17 & 0,04 & 0,11 \\
\hline & 7,5Mg@250kPa & 18 & 1,14 & 0,14 & 0,03 & 0,09 \\
\hline & 7,5Mg@350kPa & 18 & 1,17 & 0,31 & 0,07 & 0,20 \\
\hline
\end{tabular}


Tabelle A 37: Horizontale Eindringwiderstände [MPa] der Fläche NRW $_{\text {konv }}$ im Jahr 2008 nach Messtiefenbereich und Belastungsvariante

\begin{tabular}{|c|c|c|c|c|c|c|}
\hline MTB [cm] & Variante & $\mathrm{n}$ & M & SD & SED & $\mathrm{GD}_{5 \%}$ \\
\hline \multirow[t]{8}{*}{$5-14$} & Kontrolle & 18 & 0,52 & 0,06 & 0,02 & 0,04 \\
\hline & nur Schlepper & 18 & 0,57 & 0,04 & 0,01 & 0,02 \\
\hline & 3,3Mg@50kPa & 18 & 0,57 & 0,05 & 0,01 & 0,03 \\
\hline & 3,3Mg@160kPa & 18 & 0,60 & 0,04 & 0,01 & 0,03 \\
\hline & 6,3Mg@160kPa & 18 & 0,62 & 0,07 & 0,02 & 0,05 \\
\hline & 6,3Mg@250kPa & 18 & 0,65 & 0,05 & 0,01 & 0,03 \\
\hline & 7,5Mg@250kPa & 18 & 0,69 & 0,07 & 0,02 & 0,04 \\
\hline & 7,5Mg@350kPa & 18 & 0,69 & 0,10 & 0,02 & 0,06 \\
\hline \multirow[t]{8}{*}{$18-27$} & Kontrolle & 18 & 0,60 & 0,06 & 0,01 & 0,04 \\
\hline & nur Schlepper & 18 & 0,59 & 0,03 & 0,01 & 0,02 \\
\hline & 3,3Mg@50kPa & 18 & 0,62 & 0,03 & 0,01 & 0,02 \\
\hline & 3,3Mg@160kPa & 18 & 0,63 & 0,04 & 0,01 & 0,03 \\
\hline & 6,3Mg@160kPa & 18 & 0,59 & 0,03 & 0,01 & 0,02 \\
\hline & 6,3Mg@250kPa & 18 & 0,64 & 0,04 & 0,01 & 0,03 \\
\hline & 7,5Mg@250kPa & 18 & 0,65 & 0,06 & 0,01 & 0,04 \\
\hline & 7,5Mg@350kPa & 18 & 0,64 & 0,03 & 0,01 & 0,02 \\
\hline \multirow[t]{8}{*}{$30-39$} & Kontrolle & 18 & 1,02 & 0,10 & 0,02 & 0,07 \\
\hline & nur Schlepper & 18 & 0,95 & 0,09 & 0,02 & 0,06 \\
\hline & 3,3Mg@50kPa & 18 & 0,96 & 0,13 & 0,03 & 0,08 \\
\hline & 3,3Mg@160kPa & 18 & 0,93 & 0,07 & 0,02 & 0,05 \\
\hline & 6,3Mg@160kPa & 18 & 0,86 & 0,14 & 0,03 & 0,09 \\
\hline & 6,3Mg@250kPa & 18 & 0,82 & 0,11 & 0,03 & 0,07 \\
\hline & 7,5Mg@250kPa & 18 & 0,87 & 0,25 & 0,06 & 0,17 \\
\hline & 7,5Mg@350kPa & 18 & 0,81 & 0,10 & 0,02 & 0,06 \\
\hline
\end{tabular}


Tabelle A 38: Horizontale Eindringwiderstände 2008 nach Fläche, Messtiefe und Radlast unter Berücksichtigung des Sonderbereiches Geo

\begin{tabular}{|c|c|c|c|c|c|}
\hline Fläche & MTB [cm] & $\mathrm{RL}[\mathrm{Mg}]$ & $\mathrm{n}$ & $M$ & SD \\
\hline \multirow[t]{15}{*}{$\mathrm{SH}_{\text {kons }}$} & \multirow[t]{5}{*}{$5-14$} & unbefahren & 9 & 0,51 & 0,03 \\
\hline & & 3,3 & 24 & 0,55 & 0,13 \\
\hline & & 6,3 & 24 & 0,52 & 0,17 \\
\hline & & 7,5 & 30 & 0,60 & 0,09 \\
\hline & & Sonderbereich Geo & 3 & 0,96 & 0,14 \\
\hline & \multirow[t]{5}{*}{$18-27$} & unbefahren & 9 & 0,76 & 0,11 \\
\hline & & 3,3 & 24 & 0,86 & 0,13 \\
\hline & & 6,3 & 24 & 0,83 & 0,09 \\
\hline & & 7,5 & 30 & 0,95 & 0,12 \\
\hline & & Sonderbereich Geo & 3 & 1,11 & 0,30 \\
\hline & \multirow[t]{5}{*}{$30-39$} & unbefahren & 9 & 0,93 & 0,23 \\
\hline & & 3,3 & 24 & 1,14 & 0,27 \\
\hline & & 6,3 & 24 & 1,10 & 0,15 \\
\hline & & 7,5 & 30 & 1,15 & 0,15 \\
\hline & & Sonderbereich Geo & 3 & 1,29 & 0,33 \\
\hline \multirow[t]{15}{*}{$\mathrm{SH}_{\mathrm{konv}}$} & \multirow[t]{5}{*}{$5-14$} & unbefahren & 18 & 0,46 & 0,12 \\
\hline & & 3,3 & 24 & 0,52 & 0,12 \\
\hline & & 6,3 & 24 & 0,51 & 0,11 \\
\hline & & 7,5 & 30 & 0,52 & 0,14 \\
\hline & & Sonderbereich Geo & 3 & 0,76 & 0,02 \\
\hline & \multirow[t]{5}{*}{$18-27$} & unbefahren & 18 & 0,62 & 0,16 \\
\hline & & 3,3 & 24 & 0,63 & 0,10 \\
\hline & & 6,3 & 24 & 0,70 & 0,15 \\
\hline & & 7,5 & 30 & 0,72 & 0,13 \\
\hline & & Sonderbereich Geo & 3 & 0,71 & 0,06 \\
\hline & \multirow[t]{5}{*}{$30-39$} & unbefahren & 18 & 1,39 & 0,17 \\
\hline & & 3,3 & 24 & 1,39 & 0,39 \\
\hline & & 6,3 & 24 & 1,27 & 0,15 \\
\hline & & 7,5 & 30 & 1,40 & 0,47 \\
\hline & & Sonderbereich Geo & 3 & 1,12 & 0,28 \\
\hline \multirow[t]{5}{*}{$\mathrm{NRW}_{\text {kons }}$} & \multirow[t]{5}{*}{$5-14$} & unbefahren & 24 & 0,70 & 0,10 \\
\hline & & 3,3 & 24 & 0,74 & 0,07 \\
\hline & & 6,3 & 24 & 0,70 & 0,06 \\
\hline & & 7,5 & 36 & 0,73 & 0,08 \\
\hline & & Sonderbereich Geo & 18 & 0,83 & 0,08 \\
\hline
\end{tabular}




\begin{tabular}{|c|c|c|c|c|c|}
\hline & $18-27$ & unbefahren & 24 & 0,74 & 0,06 \\
\hline & & 3,3 & 24 & 0,75 & 0,08 \\
\hline & & 6,3 & 24 & 0,76 & 0,10 \\
\hline & & 7,5 & 36 & 0,80 & 0,09 \\
\hline & & Sonderbereich Geo & 18 & 0,92 & 0,07 \\
\hline & $30-39$ & unbefahren & 24 & 0,72 & 0,14 \\
\hline & & 3,3 & 24 & 0,65 & 0,07 \\
\hline & & 6,3 & 24 & 0,63 & 0,08 \\
\hline & & 7,5 & 36 & 0,64 & 0,11 \\
\hline & & Sonderbereich Geo & 18 & 0,78 & 0,10 \\
\hline $\mathrm{NRW}_{\text {konv }}$ & $5-14$ & unbefahren & 18 & 0,52 & 0,06 \\
\hline & & nur Schlepper & 18 & 0,57 & 0,04 \\
\hline & & 3,3 & 36 & 0,59 & 0,05 \\
\hline & & 6,3 & 36 & 0,64 & 0,06 \\
\hline & & 7,5 & 36 & 0,69 & 0,08 \\
\hline & & Sonderbereich Geo & 15 & 0,76 & 0,08 \\
\hline & $18-27$ & unbefahren & 18 & 0,60 & 0,06 \\
\hline & & nur Schlepper & 18 & 0,59 & 0,03 \\
\hline & & 3,3 & 36 & 0,62 & 0,03 \\
\hline & & 6,3 & 36 & 0,62 & 0,04 \\
\hline & & 7,5 & 36 & 0,65 & 0,05 \\
\hline & & Sonderbereich Geo & 15 & 0,82 & 0,11 \\
\hline & $30-39$ & unbefahren & 18 & 1,02 & 0,10 \\
\hline & & nur Schlepper & 18 & 0,95 & 0,09 \\
\hline & & 3,3 & 36 & 0,95 & 0,10 \\
\hline & & 6,3 & 36 & 0,84 & 0,12 \\
\hline & & 7,5 & 36 & 0,84 & 0,19 \\
\hline & & Sonderbereich Geo & 15 & 0,77 & 0,11 \\
\hline
\end{tabular}


Tabelle A 39: Horizontale Eindringwiderstände 2008 nach Bodenbearbeitung (BB), Messtiefenbereich (MTB) und Radlast (RL) unter Berücksichtigung des Sonderbereiches Geo

\begin{tabular}{|c|c|c|c|c|c|}
\hline BB & MTB [cm] & $\mathrm{RL}[\mathrm{Mg}]$ & $\mathrm{n}$ & M & SD \\
\hline \multirow[t]{15}{*}{ konservierend } & \multirow[t]{5}{*}{$5-14$} & unbefahren & 33 & 0,64 & 0,12 \\
\hline & & 3,3 & 48 & 0,65 & 0,14 \\
\hline & & 6,3 & 48 & 0,61 & 0,16 \\
\hline & & 7,5 & 66 & 0,67 & 0,10 \\
\hline & & Sonderbereich GEO & 21 & 0,85 & 0,10 \\
\hline & \multirow[t]{5}{*}{$18-27$} & unbefahren & 33 & 0,75 & 0,08 \\
\hline & & 3,3 & 48 & 0,81 & 0,12 \\
\hline & & 6,3 & 48 & 0,79 & 0,10 \\
\hline & & 7,5 & 66 & 0,87 & 0,13 \\
\hline & & Sonderbereich GEO & 21 & 0,94 & 0,14 \\
\hline & \multirow[t]{5}{*}{$30-39$} & unbefahren & 33 & 0,78 & 0,19 \\
\hline & & 3,3 & 48 & 0,90 & 0,31 \\
\hline & & 6,3 & 48 & 0,87 & 0,26 \\
\hline & & 7,5 & 66 & 0,87 & 0,29 \\
\hline & & Sonderbereich GEO & 21 & 0,85 & 0,23 \\
\hline \multirow[t]{18}{*}{ konventionell } & \multirow[t]{6}{*}{$5-14$} & unbefahren & 36 & 0,49 & 0,10 \\
\hline & & nur Schlepper & 18 & 0,57 & 0,04 \\
\hline & & 3,3 & 60 & 0,56 & 0,09 \\
\hline & & 6,3 & 60 & 0,58 & 0,11 \\
\hline & & 7,5 & 66 & 0,61 & 0,14 \\
\hline & & Sonderbereich GEO & 18 & 0,76 & 0,07 \\
\hline & \multirow[t]{6}{*}{$18-27$} & unbefahren & 36 & 0,61 & 0,12 \\
\hline & & nur Schlepper & 18 & 0,59 & 0,03 \\
\hline & & 3,3 & 60 & 0,63 & 0,07 \\
\hline & & 6,3 & 60 & 0,65 & 0,11 \\
\hline & & 7,5 & 66 & 0,68 & 0,10 \\
\hline & & Sonderbereich GEO & 18 & 0,81 & 0,11 \\
\hline & \multirow[t]{6}{*}{$30-39$} & unbefahren & 36 & 1,20 & 0,23 \\
\hline & & nur Schlepper & 18 & 0,95 & 0,09 \\
\hline & & 3,3 & 60 & 1,13 & 0,34 \\
\hline & & 6,3 & 60 & 1,01 & 0,25 \\
\hline & & 7,5 & 66 & 1,10 & 0,45 \\
\hline & & Sonderbereich GEO & 18 & 0,82 & 0,19 \\
\hline
\end{tabular}


Tabelle A 40: Effekt des Faktors Radlast bei $160 \mathrm{kPa}$ Reifeninnendruck auf die horizontalen Eindringwiderstände [MPa] nach Jahr, Fläche und Messtiefenbereich

\begin{tabular}{|c|c|c|c|c|c|c|c|c|}
\hline Jahr & Fläche & MTB [cm] & Variante & $\mathrm{n}$ & M & SD & SED & $\mathrm{GD}_{5 \%}$ \\
\hline \multirow[t]{24}{*}{2006} & \multirow[t]{6}{*}{$\mathrm{SH}_{\text {kons }}$} & \multirow[t]{2}{*}{$5-14$} & 3,3Mg@160kPa & 12 & 0,38 & 0,06 & 0,02 & 0,05 \\
\hline & & & 6,3Mg@160kPa & 10 & 0,45 & 0,05 & 0,02 & 0,04 \\
\hline & & \multirow[t]{2}{*}{$18-27$} & 3,3Mg@160kPa & 12 & 0,73 & 0,08 & 0,02 & 0,07 \\
\hline & & & 6,3Mg@160kPa & 10 & 0,91 & 0,08 & 0,03 & 0,07 \\
\hline & & \multirow[t]{2}{*}{$30-39$} & 3,3Mg@160kPa & 12 & 1,27 & 0,16 & 0,05 & 0,13 \\
\hline & & & 6,3Mg@160kPa & 10 & 1,35 & 0,24 & 0,08 & 0,21 \\
\hline & \multirow[t]{6}{*}{$\mathrm{SH}_{\text {konv }}$} & \multirow[t]{2}{*}{$5-14$} & 3,3Mg@160kPa & 11 & 0,85 & 0,13 & 0,04 & 0,11 \\
\hline & & & 6,3Mg@160kPa & 12 & 0,81 & 0,18 & 0,05 & 0,14 \\
\hline & & \multirow[t]{2}{*}{$18-27$} & 3,3Mg@160kPa & 11 & 0,91 & 0,17 & 0,05 & 0,14 \\
\hline & & & 6,3Mg@160kPa & 12 & 0,88 & 0,12 & 0,04 & 0,10 \\
\hline & & \multirow[t]{2}{*}{$30-39$} & 3,3Mg@160kPa & 11 & 1,85 & 0,31 & 0,09 & 0,26 \\
\hline & & & 6,3Mg@160kPa & 12 & 1,74 & 0,46 & 0,13 & 0,37 \\
\hline & \multirow[t]{6}{*}{$\mathrm{NRW}_{\text {kons }}$} & \multirow[t]{2}{*}{$5-14$} & 3,3Mg@160kPa & 12 & 0,74 & 0,07 & 0,02 & 0,05 \\
\hline & & & 6,3Mg@160kPa & 12 & 0,81 & 0,07 & 0,02 & 0,06 \\
\hline & & \multirow[t]{2}{*}{$18-27$} & 3,3Mg@160kPa & 12 & 1,05 & 0,14 & 0,04 & 0,11 \\
\hline & & & 6,3Mg@160kPa & 12 & 1,12 & 0,12 & 0,03 & 0,10 \\
\hline & & \multirow[t]{2}{*}{$30-39$} & 3,3Mg@160kPa & 12 & 0,87 & 0,10 & 0,03 & 0,08 \\
\hline & & & 6,3Mg@160kPa & 12 & 0,90 & 0,11 & 0,03 & 0,09 \\
\hline & \multirow[t]{6}{*}{$\mathrm{NRW}_{\text {konv }}$} & \multirow[t]{2}{*}{$5-14$} & 3,3Mg@160kPa & 18 & 0,61 & 0,04 & 0,01 & 0,03 \\
\hline & & & 6,3Mg@160kPa & 18 & 0,63 & 0,03 & 0,01 & 0,02 \\
\hline & & \multirow[t]{2}{*}{$18-27$} & 3,3Mg@160kPa & 18 & 0,65 & 0,06 & 0,02 & 0,04 \\
\hline & & & 6,3Mg@160kPa & 18 & 0,68 & 0,06 & 0,01 & 0,04 \\
\hline & & \multirow[t]{2}{*}{$30-39$} & 3,3Mg@160kPa & 18 & 0,93 & 0,27 & 0,06 & 0,18 \\
\hline & & & 6,3Mg@160kPa & 18 & 1,05 & 0,26 & 0,06 & 0,17 \\
\hline \multirow[t]{10}{*}{2007} & \multirow[t]{6}{*}{$\mathrm{SH}_{\mathrm{kons}}$} & \multirow[t]{2}{*}{$5-14$} & 3,3Mg@160kPa & 12 & 0,63 & 0,18 & 0,05 & 0,14 \\
\hline & & & 6,3Mg@160kPa & 12 & 0,60 & 0,12 & 0,03 & 0,10 \\
\hline & & \multirow[t]{2}{*}{$18-27$} & 3,3Mg@160kPa & 12 & 1,04 & 0,10 & 0,03 & 0,08 \\
\hline & & & 6,3Mg@160kPa & 12 & 1,02 & 0,09 & 0,03 & 0,07 \\
\hline & & \multirow[t]{2}{*}{$30-39$} & 3,3Mg@160kPa & 12 & 1,31 & 0,16 & 0,05 & 0,13 \\
\hline & & & 6,3Mg@160kPa & 12 & 1,37 & 0,15 & 0,04 & 0,12 \\
\hline & \multirow[t]{4}{*}{$\mathrm{SH}_{\text {konv }}$} & \multirow[t]{2}{*}{$5-14$} & 3,3Mg@160kPa & 12 & 0,82 & 0,14 & 0,04 & 0,12 \\
\hline & & & 6,3Mg@160kPa & 12 & 0,75 & 0,10 & 0,03 & 0,08 \\
\hline & & \multirow[t]{2}{*}{$18-27$} & 3,3Mg@160kPa & 12 & 0,93 & 0,14 & 0,04 & 0,12 \\
\hline & & & 6,3Mg@160kPa & 12 & 1,12 & 0,20 & 0,06 & 0,16 \\
\hline
\end{tabular}




\begin{tabular}{|c|c|c|c|c|c|c|c|c|}
\hline & $N R W_{\text {kons }}$ & $5-14$ & 3,3Mg@160kPa & 12 & 0,80 & 0,08 & 0,02 & 0,06 \\
\hline & & & 6,3Mg@160kPa & 12 & 0,82 & 0,09 & 0,03 & 0,07 \\
\hline & & $18-27$ & 3,3Mg@160kPa & 12 & 1,02 & 0,10 & 0,03 & 0,08 \\
\hline & & & 6,3Mg@160kPa & 12 & 1,08 & 0,08 & 0,02 & 0,06 \\
\hline & & $30-39$ & 3,3Mg@160kPa & 12 & 0,99 & 0,14 & 0,04 & 0,11 \\
\hline & & & 6,3Mg@160kPa & 12 & 0,98 & 0,15 & 0,04 & 0,12 \\
\hline & $\mathrm{NRW}_{\text {konv }}$ & $5-14$ & 3,3Mg@160kPa & 18 & 0,60 & 0,06 & 0,01 & 0,04 \\
\hline & & & 6,3Mg@160kPa & 18 & 0,56 & 0,05 & 0,01 & 0,04 \\
\hline & & $18-27$ & 3,3Mg@160kPa & 18 & 0,64 & 0,04 & 0,01 & 0,02 \\
\hline & & & 6,3Mg@160kPa & 18 & 0,66 & 0,06 & 0,01 & 0,04 \\
\hline & & $30-39$ & 3,3Mg@160kPa & 18 & 1,05 & 0,16 & 0,04 & 0,11 \\
\hline & & & 6,3Mg@160kPa & 18 & 1,18 & 0,17 & 0,04 & 0,11 \\
\hline 2008 & $\mathrm{SH}_{\text {kons }}$ & $5-14$ & 3,3Mg@160kPa & 12 & 0,52 & 0,12 & 0,04 & 0,10 \\
\hline & & & 6,3Mg@160kPa & 12 & 0,50 & 0,19 & 0,06 & 0,15 \\
\hline & & $18-27$ & 3,3Mg@160kPa & 12 & 0,81 & 0,11 & 0,03 & 0,09 \\
\hline & & & 6,3Mg@160kPa & 12 & 0,82 & 0,08 & 0,02 & 0,07 \\
\hline & & $30-39$ & 3,3Mg@160kPa & 12 & 1,10 & 0,31 & 0,09 & 0,25 \\
\hline & & & 6,3Mg@160kPa & 12 & 1,15 & 0,13 & 0,04 & 0,10 \\
\hline & $\mathrm{SH}_{\mathrm{konv}}$ & $5-14$ & 3,3Mg@160kPa & 12 & 0,53 & 0,12 & 0,03 & 0,09 \\
\hline & & & 6,3Mg@160kPa & 12 & 0,49 & 0,13 & 0,04 & 0,10 \\
\hline & & $18-27$ & 3,3Mg@160kPa & 12 & 0,62 & 0,11 & 0,03 & 0,09 \\
\hline & & & 6,3Mg@160kPa & 12 & 0,69 & 0,19 & 0,06 & 0,16 \\
\hline & & $30-39$ & 3,3Mg@160kPa & 12 & 1,28 & 0,28 & 0,08 & 0,22 \\
\hline & & & 6,3Mg@160kPa & 12 & 1,26 & 0,16 & 0,05 & 0,13 \\
\hline & NRW & $5-14$ & 3,3Mg@160kPa & 12 & 0,75 & 0,07 & 0,02 & 0,05 \\
\hline & & & 6,3Mg@160kPa & 12 & 0,71 & 0,05 & 0,01 & 0,04 \\
\hline & & $18-27$ & 3,3Mg@160kPa & 12 & 0,71 & 0,06 & 0,02 & 0,05 \\
\hline & & & 6,3Mg@160kPa & 12 & 0,68 & 0,05 & 0,01 & 0,04 \\
\hline & & $30-39$ & 3,3Mg@160kPa & 12 & 0,64 & 0,08 & 0,02 & 0,06 \\
\hline & & & 6,3Mg@160kPa & 12 & 0,63 & 0,06 & 0,02 & 0,05 \\
\hline & $\mathrm{NRW}_{\text {konv }}$ & $5-14$ & 3,3Mg@160kPa & 18 & 0,60 & 0,04 & 0,01 & 0,03 \\
\hline & & & 6,3Mg@160kPa & 18 & 0,62 & 0,07 & 0,02 & 0,05 \\
\hline & & $18-27$ & 3,3Mg@160kPa & 18 & 0,63 & 0,04 & 0,01 & 0,03 \\
\hline & & & 6,3Mg@160kPa & 18 & 0,59 & 0,03 & 0,01 & 0,02 \\
\hline & & $30-39$ & 3,3Mg@160kPa & 18 & 0,93 & 0,07 & 0,02 & 0,05 \\
\hline & & & 6,3Mg@160kPa & 18 & 0,86 & 0,14 & 0,03 & 0,09 \\
\hline
\end{tabular}


Tabelle A 41: Effekt des Faktors Radlast bei $250 \mathrm{kPa}$ Reifeninnendruck auf die horizontalen Eindringwiderstände [MPa] nach Jahr, Fläche und Messtiefenbereich

\begin{tabular}{|c|c|c|c|c|c|c|c|c|}
\hline Jahr & Fläche & MTB [cm] & Variante & $\mathrm{n}$ & M & SD & SED & $\mathrm{GD}_{5 \%}$ \\
\hline \multirow[t]{24}{*}{2006} & \multirow[t]{6}{*}{$\mathrm{SH}_{\text {kons }}$} & \multirow[t]{2}{*}{$5-14$} & 6,3Mg@250kPa & 12 & 0,41 & 0,07 & 0,02 & 0,06 \\
\hline & & & 7,5Mg@250kPa & 12 & 0,36 & 0,04 & 0,01 & 0,03 \\
\hline & & \multirow[t]{2}{*}{$18-27$} & 6,3Mg@250kPa & 12 & 0,79 & 0,09 & 0,03 & 0,08 \\
\hline & & & 7,5Mg@250kPa & 12 & 0,82 & 0,09 & 0,03 & 0,07 \\
\hline & & \multirow[t]{2}{*}{$30-39$} & 6,3Mg@250kPa & 12 & 1,29 & 0,13 & 0,04 & 0,10 \\
\hline & & & 7,5Mg@250kPa & 12 & 1,32 & 0,12 & 0,03 & 0,09 \\
\hline & \multirow[t]{6}{*}{$\mathrm{SH}_{\text {konv }}$} & \multirow[t]{2}{*}{$5-14$} & 6,3Mg@250kPa & 12 & 0,73 & 0,22 & 0,06 & 0,18 \\
\hline & & & 7,5Mg@250kPa & 12 & 0,84 & 0,14 & 0,04 & 0,12 \\
\hline & & \multirow[t]{2}{*}{$18-27$} & 6,3Mg@250kPa & 12 & 0,89 & 0,13 & 0,04 & 0,11 \\
\hline & & & 7,5Mg@250kPa & 12 & 0,90 & 0,17 & 0,05 & 0,14 \\
\hline & & \multirow[t]{2}{*}{$30-39$} & 6,3Mg@250kPa & 12 & 1,98 & 0,29 & 0,08 & 0,24 \\
\hline & & & 7,5Mg@250kPa & 12 & 2,02 & 0,87 & 0,25 & 0,70 \\
\hline & \multirow[t]{6}{*}{$\mathrm{NRW}_{\text {kons }}$} & \multirow[t]{2}{*}{$5-14$} & 6,3Mg@250kPa & 12 & 0,78 & 0,12 & 0,03 & 0,10 \\
\hline & & & 7,5Mg@250kPa & 12 & 0,75 & 0,06 & 0,02 & 0,05 \\
\hline & & \multirow[t]{2}{*}{$18-27$} & 6,3Mg@250kPa & 12 & 1,08 & 0,12 & 0,03 & 0,10 \\
\hline & & & 7,5Mg@250kPa & 12 & 1,11 & 0,10 & 0,03 & 0,08 \\
\hline & & \multirow[t]{2}{*}{$30-39$} & 6,3Mg@250kPa & 12 & 0,87 & 0,11 & 0,03 & 0,09 \\
\hline & & & 7,5Mg@250kPa & 12 & 0,90 & 0,19 & 0,06 & 0,15 \\
\hline & \multirow[t]{6}{*}{$\mathrm{NRW}_{\text {konv }}$} & \multirow[t]{2}{*}{$5-14$} & 6,3Mg@250kPa & 18 & 0,61 & 0,05 & 0,01 & 0,04 \\
\hline & & & 7,5Mg@250kPa & 18 & 0,60 & 0,06 & 0,01 & 0,04 \\
\hline & & \multirow[t]{2}{*}{$18-27$} & 6,3Mg@250kPa & 18 & 0,67 & 0,09 & 0,02 & 0,06 \\
\hline & & & 7,5Mg@250kPa & 18 & 0,64 & 0,10 & 0,02 & 0,06 \\
\hline & & \multirow[t]{2}{*}{$30-39$} & 6,3Mg@250kPa & 18 & 1,01 & 0,34 & 0,08 & 0,22 \\
\hline & & & 7,5Mg@250kPa & 18 & 1,07 & 0,23 & 0,05 & 0,15 \\
\hline \multirow[t]{10}{*}{2007} & \multirow[t]{6}{*}{$\mathrm{SH}_{\text {kons }}$} & \multirow[t]{2}{*}{$5-14$} & 6,3Mg@250kPa & 12 & 0,51 & 0,15 & 0,04 & 0,12 \\
\hline & & & 7,5Mg@250kPa & 12 & 0,56 & 0,21 & 0,06 & 0,17 \\
\hline & & \multirow[t]{2}{*}{$18-27$} & 6,3Mg@250kPa & 12 & 1,00 & 0,16 & 0,05 & 0,13 \\
\hline & & & 7,5Mg@250kPa & 12 & 1,05 & 0,20 & 0,06 & 0,16 \\
\hline & & \multirow[t]{2}{*}{$30-39$} & 6,3Mg@250kPa & 12 & 1,48 & 0,29 & 0,09 & 0,24 \\
\hline & & & 7,5Mg@250kPa & 12 & 1,45 & 0,26 & 0,08 & 0,21 \\
\hline & \multirow[t]{4}{*}{$\mathrm{SH}_{\text {konv }}$} & \multirow[t]{2}{*}{$5-14$} & 6,3Mg@250kPa & 12 & 0,85 & 0,17 & 0,05 & 0,14 \\
\hline & & & 7,5Mg@250kPa & 12 & 0,78 & 0,22 & 0,06 & 0,18 \\
\hline & & \multirow[t]{2}{*}{$18-27$} & 6,3Mg@250kPa & 12 & 1,07 & 0,13 & 0,04 & 0,11 \\
\hline & & & 7,5Mg@250kPa & 12 & 1,05 & 0,11 & 0,03 & 0,09 \\
\hline
\end{tabular}




\begin{tabular}{|c|c|c|c|c|c|c|c|c|}
\hline \multirow{6}{*}{\multicolumn{2}{|c|}{$\mathrm{NRW}_{\text {kons }}$}} & \multirow[t]{2}{*}{$5-14$} & 6,3Mg@250kPa & 12 & 0,78 & 0,15 & 0,04 & 0,12 \\
\hline & & & 7,5Mg@250kPa & 12 & 0,77 & 0,12 & 0,04 & 0,10 \\
\hline & & \multirow[t]{2}{*}{$18-27$} & 6,3Mg@250kPa & 12 & 1,02 & 0,14 & 0,04 & 0,11 \\
\hline & & & 7,5Mg@250kPa & 12 & 1,08 & 0,07 & 0,02 & 0,06 \\
\hline & & \multirow[t]{2}{*}{$30-39$} & 6,3Mg@250kPa & 12 & 1,06 & 0,17 & 0,05 & 0,14 \\
\hline & & & 7,5Mg@250kPa & 12 & 1,01 & 0,15 & 0,04 & 0,12 \\
\hline & \multirow[t]{6}{*}{$\mathrm{NRW}_{\text {konv }}$} & \multirow[t]{2}{*}{$5-14$} & 6,3Mg@250kPa & 18 & 0,60 & 0,11 & 0,03 & 0,07 \\
\hline & & & 7,5Mg@250kPa & 18 & 0,56 & 0,08 & 0,02 & 0,05 \\
\hline & & \multirow[t]{2}{*}{$18-27$} & 6,3Mg@250kPa & 18 & 0,69 & 0,05 & 0,01 & 0,03 \\
\hline & & & 7,5Mg@250kPa & 18 & 0,71 & 0,06 & 0,01 & 0,04 \\
\hline & & \multirow[t]{2}{*}{$30-39$} & 6,3Mg@250kPa & 18 & 1,09 & 0,17 & 0,04 & 0,11 \\
\hline & & & 7,5Mg@250kPa & 18 & 1,14 & 0,14 & 0,03 & 0,09 \\
\hline \multirow[t]{26}{*}{2008} & \multirow[t]{6}{*}{$\mathrm{SH}_{\text {kons }}$} & \multirow[t]{2}{*}{$5-14$} & 6,3Mg@250kPa & 12 & 0,53 & 0,16 & 0,05 & 0,13 \\
\hline & & & 7,5Mg@250kPa & 12 & 0,59 & 0,05 & 0,02 & 0,04 \\
\hline & & \multirow[t]{2}{*}{$18-27$} & 6,3Mg@250kPa & 12 & 0,84 & 0,11 & 0,03 & 0,08 \\
\hline & & & 7,5Mg@250kPa & 12 & 0,93 & 0,09 & 0,03 & 0,07 \\
\hline & & \multirow[t]{2}{*}{$30-39$} & 6,3Mg@250kPa & 12 & 1,05 & 0,15 & 0,04 & 0,12 \\
\hline & & & 7,5Mg@250kPa & 12 & 1,18 & 0,13 & 0,04 & 0,10 \\
\hline & \multirow[t]{6}{*}{$\mathrm{SH}_{\mathrm{konv}}$} & \multirow[t]{2}{*}{$5-14$} & 6,3Mg@250kPa & 12 & 0,53 & 0,10 & 0,03 & 0,08 \\
\hline & & & 7,5Mg@250kPa & 12 & 0,50 & 0,12 & 0,04 & 0,10 \\
\hline & & \multirow[t]{2}{*}{$18-27$} & 6,3Mg@250kPa & 12 & 0,71 & 0,09 & 0,02 & 0,07 \\
\hline & & & 7,5Mg@250kPa & 12 & 0,65 & 0,07 & 0,02 & 0,06 \\
\hline & & \multirow[t]{2}{*}{$30-39$} & 6,3Mg@250kPa & 12 & 1,28 & 0,15 & 0,04 & 0,12 \\
\hline & & & 7,5Mg@250kPa & 12 & 1,28 & 0,25 & 0,07 & 0,20 \\
\hline & \multirow[t]{8}{*}{$\mathrm{NRW}_{\text {kons }}$} & \multirow[t]{2}{*}{$5-14$} & 6,3Mg@250kPa & 12 & 0,68 & 0,07 & 0,02 & 0,06 \\
\hline & & & 7,5Mg@250kPa & 12 & 0,77 & 0,06 & 0,02 & 0,05 \\
\hline & & \multirow[t]{2}{*}{$18-27$} & 6,3Mg@250kPa & 12 & 0,83 & 0,07 & 0,02 & 0,06 \\
\hline & & & 7,5Mg@250kPa & 12 & 0,77 & 0,06 & 0,02 & 0,05 \\
\hline & & \multirow[t]{2}{*}{$30-39$} & 6,3Mg@250kPa & 12 & 0,63 & 0,11 & 0,03 & 0,09 \\
\hline & & & 7,5Mg@250kPa & 12 & 0,67 & 0,04 & 0,01 & 0,03 \\
\hline & & \multirow[t]{2}{*}{ Insgesamt } & 6,3Mg@250kPa & 36 & 0,72 & 0,12 & 0,02 & 0,05 \\
\hline & & & 7,5Mg@250kPa & 36 & 0,74 & 0,07 & 0,01 & 0,03 \\
\hline & \multirow[t]{6}{*}{$\mathrm{NRW}_{\text {konv }}$} & \multirow[t]{2}{*}{$5-14$} & 6,3Mg@250kPa & 18 & 0,65 & 0,05 & 0,01 & 0,03 \\
\hline & & & 7,5Mg@250kPa & 18 & 0,69 & 0,07 & 0,02 & 0,04 \\
\hline & & \multirow[t]{2}{*}{$18-27$} & 6,3Mg@250kPa & 18 & 0,64 & 0,04 & 0,01 & 0,03 \\
\hline & & & 7,5Mg@250kPa & 18 & 0,65 & 0,06 & 0,01 & 0,04 \\
\hline & & \multirow[t]{2}{*}{$30-39$} & 6,3Mg@250kPa & 18 & 0,82 & 0,11 & 0,03 & 0,07 \\
\hline & & & 7,5Mg@250kPa & 18 & 0,87 & 0,25 & 0,06 & 0,17 \\
\hline
\end{tabular}


Tabelle A 42: Effekt des Faktors Reifeninnendruck bei 3,3 Mg Radlast auf die horizontalen Eindringwiderstände [MPa] nach Jahr, Fläche und Messtiefenbereich

\begin{tabular}{|c|c|c|c|c|c|c|c|c|}
\hline Jahr & Fläche & MTB [cm] & Variante & $\mathrm{n}$ & $M$ & SD & SED & $\mathrm{GD}_{5 \%}$ \\
\hline \multirow[t]{26}{*}{2006} & \multirow[t]{6}{*}{$\mathrm{SH}_{\text {kons }}$} & \multirow[t]{2}{*}{$5-14$} & 3,3Mg@50kPa & 12 & 0,39 & 0,04 & 0,01 & 0,03 \\
\hline & & & 3,3Mg@160kPa & 12 & 0,38 & 0,06 & 0,02 & 0,05 \\
\hline & & \multirow[t]{2}{*}{$18-27$} & 3,3Mg@50kPa & 12 & 0,80 & 0,05 & 0,01 & 0,04 \\
\hline & & & 3,3Mg@160kPa & 12 & 0,73 & 0,08 & 0,02 & 0,07 \\
\hline & & \multirow[t]{2}{*}{$30-39$} & 3,3Mg@50kPa & 12 & 1,34 & 0,18 & 0,05 & 0,14 \\
\hline & & & 3,3Mg@160kPa & 12 & 1,27 & 0,16 & 0,05 & 0,13 \\
\hline & \multirow[t]{6}{*}{$\mathrm{SH}_{\mathrm{konv}}$} & \multirow[t]{2}{*}{$5-14$} & 3,3Mg@50kPa & 12 & 0,74 & 0,17 & 0,05 & 0,14 \\
\hline & & & 3,3Mg@160kPa & 11 & 0,85 & 0,13 & 0,04 & 0,11 \\
\hline & & \multirow[t]{2}{*}{$18-27$} & 3,3Mg@50kPa & 12 & 0,82 & 0,10 & 0,03 & 0,08 \\
\hline & & & 3,3Mg@160kPa & 11 & 0,91 & 0,17 & 0,05 & 0,14 \\
\hline & & \multirow[t]{2}{*}{$30-39$} & 3,3Mg@50kPa & 12 & 1,91 & 0,38 & 0,11 & 0,30 \\
\hline & & & 3,3Mg@160kPa & 11 & 1,85 & 0,31 & 0,09 & 0,26 \\
\hline & \multirow[t]{8}{*}{$\mathrm{NRW}_{\text {kons }}$} & \multirow[t]{2}{*}{$5-14$} & 3,3Mg@50kPa & 12 & 0,76 & 0,11 & 0,03 & 0,09 \\
\hline & & & 3,3Mg@160kPa & 12 & 0,74 & 0,07 & 0,02 & 0,05 \\
\hline & & \multirow[t]{2}{*}{$18-27$} & 3,3Mg@50kPa & 12 & 1,13 & 0,14 & 0,04 & 0,11 \\
\hline & & & 3,3Mg@160kPa & 12 & 1,05 & 0,14 & 0,04 & 0,11 \\
\hline & & \multirow[t]{2}{*}{$30-39$} & 3,3Mg@50kPa & 12 & 0,93 & 0,09 & 0,03 & 0,07 \\
\hline & & & 3,3Mg@160kPa & 12 & 0,87 & 0,10 & 0,03 & 0,08 \\
\hline & & \multirow{2}{*}{ Insgesamt } & 3,3Mg@50kPa & 36 & 0,94 & 0,19 & 0,03 & 0,09 \\
\hline & & & 3,3Mg@160kPa & 36 & 0,89 & 0,16 & 0,03 & 0,08 \\
\hline & \multirow[t]{6}{*}{$\mathrm{NRW}_{\text {konv }}$} & \multirow[t]{2}{*}{$5-14$} & 3,3Mg@50kPa & 18 & 0,62 & 0,06 & 0,01 & 0,04 \\
\hline & & & 3,3Mg@160kPa & 18 & 0,61 & 0,04 & 0,01 & 0,03 \\
\hline & & \multirow[t]{2}{*}{$18-27$} & 3,3Mg@50kPa & 18 & 0,59 & 0,06 & 0,01 & 0,04 \\
\hline & & & 3,3Mg@160kPa & 18 & 0,65 & 0,06 & 0,02 & 0,04 \\
\hline & & \multirow[t]{2}{*}{$30-39$} & 3,3Mg@50kPa & 18 & 0,89 & 0,21 & 0,05 & 0,14 \\
\hline & & & 3,3Mg@160kPa & 18 & 0,93 & 0,27 & 0,06 & 0,18 \\
\hline \multirow[t]{10}{*}{2007} & \multirow[t]{6}{*}{$\mathrm{SH}_{\text {kons }}$} & \multirow[t]{2}{*}{$5-14$} & 3,3Mg@50kPa & 12 & 0,60 & 0,13 & 0,04 & 0,10 \\
\hline & & & 3,3Mg@160kPa & 12 & 0,63 & 0,18 & 0,05 & 0,14 \\
\hline & & \multirow[t]{2}{*}{$18-27$} & 3,3Mg@50kPa & 12 & 0,98 & 0,12 & 0,04 & 0,10 \\
\hline & & & 3,3Mg@160kPa & 12 & 1,04 & 0,10 & 0,03 & 0,08 \\
\hline & & \multirow[t]{2}{*}{$30-39$} & 3,3Mg@50kPa & 12 & 1,36 & 0,18 & 0,05 & 0,14 \\
\hline & & & 3,3Mg@160kPa & 12 & 1,31 & 0,16 & 0,05 & 0,13 \\
\hline & \multirow[t]{4}{*}{$\mathrm{SH}_{\mathrm{konv}}$} & \multirow[t]{2}{*}{$5-14$} & 3,3Mg@50kPa & 12 & 0,84 & 0,19 & 0,06 & 0,16 \\
\hline & & & 3,3Mg@160kPa & 12 & 0,82 & 0,14 & 0,04 & 0,12 \\
\hline & & \multirow[t]{2}{*}{$18-27$} & 3,3Mg@50kPa & 12 & 0,94 & 0,14 & 0,04 & 0,12 \\
\hline & & & 3,3Mg@160kPa & 12 & 0,93 & 0,14 & 0,04 & 0,12 \\
\hline
\end{tabular}




\begin{tabular}{|c|c|c|c|c|c|c|c|c|}
\hline & $\mathrm{NRW}_{\text {kons }}$ & $5-14$ & 3,3Mg@50kPa & 12 & 0,78 & 0,08 & 0,02 & 0,07 \\
\hline & & & 3,3Mg@160kPa & 12 & 0,80 & 0,08 & 0,02 & 0,06 \\
\hline & & $18-27$ & 3,3Mg@50kPa & 12 & 1,00 & 0,11 & 0,03 & 0,09 \\
\hline & & & 3,3Mg@160kPa & 12 & 1,02 & 0,10 & 0,03 & 0,08 \\
\hline & & $30-39$ & 3,3Mg@50kPa & 12 & 0,99 & 0,13 & 0,04 & 0,11 \\
\hline & & & 3,3Mg@160kPa & 12 & 0,99 & 0,14 & 0,04 & 0,11 \\
\hline & $\mathrm{NRW}_{\text {konv }}$ & $5-14$ & 3,3Mg@50kPa & 18 & 0,63 & 0,07 & 0,02 & 0,05 \\
\hline & & & 3,3Mg@160kPa & 18 & 0,60 & 0,06 & 0,01 & 0,04 \\
\hline & & $18-27$ & 3,3Mg@50kPa & 18 & 0,67 & 0,05 & 0,01 & 0,03 \\
\hline & & & 3,3Mg@160kPa & 18 & 0,64 & 0,04 & 0,01 & 0,02 \\
\hline & & $30-39$ & 3,3Mg@50kPa & 18 & 1,15 & 0,16 & 0,04 & 0,10 \\
\hline & & & 3,3Mg@160kPa & 18 & 1,05 & 0,16 & 0,04 & 0,11 \\
\hline 2008 & $\mathrm{SH}_{\text {kons }}$ & $5-14$ & 3,3Mg@50kPa & 12 & 0,59 & 0,12 & 0,04 & 0,10 \\
\hline & & & 3,3Mg@160kPa & 12 & 0,52 & 0,12 & 0,04 & 0,10 \\
\hline & & $18-27$ & 3,3Mg@50kPa & 12 & 0,92 & 0,12 & 0,04 & 0,10 \\
\hline & & & 3,3Mg@160kPa & 12 & 0,81 & 0,11 & 0,03 & 0,09 \\
\hline & & $30-39$ & 3,3Mg@50kPa & 12 & 1,17 & 0,23 & 0,07 & 0,18 \\
\hline & & & 3,3Mg@160kPa & 12 & 1,10 & 0,31 & 0,09 & 0,25 \\
\hline & $\mathrm{SH}_{\mathrm{konv}}$ & $5-14$ & 3,3Mg@50kPa & 12 & 0,51 & 0,13 & 0,04 & 0,11 \\
\hline & & & 3,3Mg@160kPa & 12 & 0,53 & 0,12 & 0,03 & 0,09 \\
\hline & & $18-27$ & 3,3Mg@50kPa & 12 & 0,64 & 0,09 & 0,02 & 0,07 \\
\hline & & & 3,3Mg@160kPa & 12 & 0,62 & 0,11 & 0,03 & 0,09 \\
\hline & & $30-39$ & 3,3Mg@50kPa & 12 & 1,50 & 0,46 & 0,13 & 0,37 \\
\hline & & & 3,3Mg@160kPa & 12 & 1,28 & 0,28 & 0,08 & 0,22 \\
\hline & $\mathrm{NRW}_{\text {kons }}$ & $5-14$ & 3,3Mg@50kPa & 12 & 0,73 & 0,07 & 0,02 & 0,06 \\
\hline & & & 3,3Mg@160kPa & 12 & 0,75 & 0,07 & 0,02 & 0,05 \\
\hline & & $18-27$ & 3,3Mg@50kPa & 12 & 0,80 & 0,07 & 0,02 & 0,06 \\
\hline & & & 3,3Mg@160kPa & 12 & 0,71 & 0,06 & 0,02 & 0,05 \\
\hline & & $30-39$ & 3,3Mg@50kPa & 12 & 0,66 & 0,06 & 0,02 & 0,05 \\
\hline & & & 3,3Mg@160kPa & 12 & 0,64 & 0,08 & 0,02 & 0,06 \\
\hline & $\mathrm{NRW}_{\text {konv }}$ & $5-14$ & 3,3Mg@50kPa & 18 & 0,57 & 0,05 & 0,01 & 0,03 \\
\hline & & & 3,3Mg@160kPa & 18 & 0,60 & 0,04 & 0,01 & 0,03 \\
\hline & & $18-27$ & 3,3Mg@50kPa & 18 & 0,62 & 0,03 & 0,01 & 0,02 \\
\hline & & & 3,3Mg@160kPa & 18 & 0,63 & 0,04 & 0,01 & 0,03 \\
\hline & & $30-39$ & 3,3Mg@50kPa & 18 & 0,96 & 0,13 & 0,03 & 0,08 \\
\hline & & & 3,3Mg@160kPa & 18 & 0,93 & 0,07 & 0,02 & 0,05 \\
\hline
\end{tabular}


Tabelle A 43: Effekt des Faktors Reifeninnendruck bei 6,3 Mg Radlast auf die horizontalen Eindringwiderstände [MPa] nach Jahr, Fläche und Messtiefenbereich

\begin{tabular}{|c|c|c|c|c|c|c|c|c|}
\hline Jahr & Fläche & MTB [cm] & Variante & $\mathrm{n}$ & M & SD & SED & $\mathrm{GD}_{5 \%}$ \\
\hline \multirow[t]{24}{*}{2006} & \multirow[t]{6}{*}{$\mathrm{SH}_{\text {kons }}$} & \multirow[t]{2}{*}{$5-14$} & 6,3Mg@160kPa & 10 & 0,45 & 0,05 & 0,02 & 0,04 \\
\hline & & & 6,3Mg@250kPa & 12 & 0,41 & 0,07 & 0,02 & 0,06 \\
\hline & & \multirow[t]{2}{*}{$18-27$} & 6,3Mg@160kPa & 10 & 0,91 & 0,08 & 0,03 & 0,07 \\
\hline & & & 6,3Mg@250kPa & 12 & 0,79 & 0,09 & 0,03 & 0,08 \\
\hline & & \multirow[t]{2}{*}{$30-39$} & 6,3Mg@160kPa & 10 & 1,35 & 0,24 & 0,08 & 0,21 \\
\hline & & & 6,3Mg@250kPa & 12 & 1,29 & 0,13 & 0,04 & 0,10 \\
\hline & \multirow[t]{6}{*}{$\mathrm{SH}_{\text {konv }}$} & \multirow[t]{2}{*}{$5-14$} & 6,3Mg@160kPa & 12 & 0,81 & 0,18 & 0,05 & 0,14 \\
\hline & & & 6,3Mg@250kPa & 12 & 0,73 & 0,22 & 0,06 & 0,18 \\
\hline & & \multirow[t]{2}{*}{$18-27$} & 6,3Mg@160kPa & 12 & 0,88 & 0,12 & 0,04 & 0,10 \\
\hline & & & 6,3Mg@250kPa & 12 & 0,89 & 0,13 & 0,04 & 0,11 \\
\hline & & \multirow[t]{2}{*}{$30-39$} & 6,3Mg@160kPa & 12 & 1,74 & 0,46 & 0,13 & 0,37 \\
\hline & & & 6,3Mg@250kPa & 12 & 1,98 & 0,29 & 0,08 & 0,24 \\
\hline & \multirow[t]{6}{*}{$\mathrm{NRW}_{\text {kons }}$} & \multirow[t]{2}{*}{$5-14$} & 6,3Mg@160kPa & 12 & 0,81 & 0,07 & 0,02 & 0,06 \\
\hline & & & 6,3Mg@250kPa & 12 & 0,78 & 0,12 & 0,03 & 0,10 \\
\hline & & \multirow[t]{2}{*}{$18-27$} & 6,3Mg@160kPa & 12 & 1,12 & 0,12 & 0,03 & 0,10 \\
\hline & & & 6,3Mg@250kPa & 12 & 1,08 & 0,12 & 0,03 & 0,10 \\
\hline & & \multirow[t]{2}{*}{$30-39$} & 6,3Mg@160kPa & 12 & 0,90 & 0,11 & 0,03 & 0,09 \\
\hline & & & 6,3Mg@250kPa & 12 & 0,87 & 0,11 & 0,03 & 0,09 \\
\hline & \multirow[t]{6}{*}{$\mathrm{NRW}_{\text {konv }}$} & \multirow[t]{2}{*}{$5-14$} & 6,3Mg@160kPa & 18 & 0,63 & 0,03 & 0,01 & 0,02 \\
\hline & & & 6,3Mg@250kPa & 18 & 0,61 & 0,05 & 0,01 & 0,04 \\
\hline & & \multirow[t]{2}{*}{$18-27$} & 6,3Mg@160kPa & 18 & 0,68 & 0,06 & 0,01 & 0,04 \\
\hline & & & 6,3Mg@250kPa & 18 & 0,67 & 0,09 & 0,02 & 0,06 \\
\hline & & \multirow[t]{2}{*}{$30-39$} & 6,3Mg@160kPa & 18 & 1,05 & 0,26 & 0,06 & 0,17 \\
\hline & & & 6,3Mg@250kPa & 18 & 1,01 & 0,34 & 0,08 & 0,22 \\
\hline \multirow[t]{12}{*}{2007} & \multirow[t]{6}{*}{$\mathrm{SH}_{\text {kons }}$} & \multirow[t]{2}{*}{$5-14$} & 6,3Mg@160kPa & 12 & 0,60 & 0,12 & 0,03 & 0,10 \\
\hline & & & 6,3Mg@250kPa & 12 & 0,51 & 0,15 & 0,04 & 0,12 \\
\hline & & \multirow[t]{2}{*}{$18-27$} & 6,3Mg@160kPa & 12 & 1,02 & 0,09 & 0,03 & 0,07 \\
\hline & & & 6,3Mg@250kPa & 12 & 1,00 & 0,16 & 0,05 & 0,13 \\
\hline & & \multirow[t]{2}{*}{$30-39$} & 6,3Mg@160kPa & 12 & 1,37 & 0,15 & 0,04 & 0,12 \\
\hline & & & 6,3Mg@250kPa & 12 & 1,48 & 0,29 & 0,09 & 0,24 \\
\hline & \multirow[t]{4}{*}{$\mathrm{SH}_{\mathrm{konv}}$} & \multirow[t]{2}{*}{$5-14$} & 6,3Mg@160kPa & 12 & 0,75 & 0,10 & 0,03 & 0,08 \\
\hline & & & 6,3Mg@250kPa & 12 & 0,85 & 0,17 & 0,05 & 0,14 \\
\hline & & \multirow[t]{2}{*}{$18-27$} & 6,3Mg@160kPa & 12 & 1,12 & 0,20 & 0,06 & 0,16 \\
\hline & & & 6,3Mg@250kPa & 12 & 1,07 & 0,13 & 0,04 & 0,11 \\
\hline & \multirow[t]{2}{*}{$\mathrm{NRW}_{\text {kons }}$} & $5-14$ & 6,3Mg@160kPa & 12 & 0,82 & 0,09 & 0,03 & 0,07 \\
\hline & & & 6,3Mg@250kPa & 12 & 0,78 & 0,15 & 0,04 & 0,12 \\
\hline
\end{tabular}




\begin{tabular}{|c|c|c|c|c|c|c|c|c|}
\hline & & $18-27$ & 6,3Mg@160kPa & 12 & 1,08 & 0,08 & 0,02 & 0,06 \\
\hline & & & 6,3Mg@250kPa & 12 & 1,02 & 0,14 & 0,04 & 0,11 \\
\hline & & $30-39$ & 6,3Mg@160kPa & 12 & 0,98 & 0,15 & 0,04 & 0,12 \\
\hline & & & 6,3Mg@250kPa & 12 & 1,06 & 0,17 & 0,05 & 0,14 \\
\hline & $\mathrm{NRW}_{\text {konv }}$ & $5-14$ & 6,3Mg@160kPa & 18 & 0,56 & 0,05 & 0,01 & 0,04 \\
\hline & & & 6,3Mg@250kPa & 18 & 0,60 & 0,11 & 0,03 & 0,07 \\
\hline & & $18-27$ & 6,3Mg@160kPa & 18 & 0,66 & 0,06 & 0,01 & 0,04 \\
\hline & & & 6,3Mg@250kPa & 18 & 0,69 & 0,05 & 0,01 & 0,03 \\
\hline & & $30-39$ & 6,3Mg@160kPa & 18 & 1,18 & 0,17 & 0,04 & 0,11 \\
\hline & & & 6,3Mg@250kPa & 18 & 1,09 & 0,17 & 0,04 & 0,11 \\
\hline 2008 & $\mathrm{SH}_{\mathrm{kons}}$ & $5-14$ & 6,3Mg@160kPa & 12 & 0,50 & 0,19 & 0,06 & 0,15 \\
\hline & & & 6,3Mg@250kPa & 12 & 0,53 & 0,16 & 0,05 & 0,13 \\
\hline & & $18-27$ & 6,3Mg@160kPa & 12 & 0,82 & 0,08 & 0,02 & 0,07 \\
\hline & & & 6,3Mg@250kPa & 12 & 0,84 & 0,11 & 0,03 & 0,08 \\
\hline & & $30-39$ & 6,3Mg@160kPa & 12 & 1,15 & 0,13 & 0,04 & 0,10 \\
\hline & & & 6,3Mg@250kPa & 12 & 1,05 & 0,15 & 0,04 & 0,12 \\
\hline & $\mathrm{SH}_{\text {konv }}$ & $5-14$ & 6,3Mg@160kPa & 12 & 0,49 & 0,13 & 0,04 & 0,10 \\
\hline & & & 6,3Mg@250kPa & 12 & 0,53 & 0,10 & 0,03 & 0,08 \\
\hline & & $18-27$ & 6,3Mg@160kPa & 12 & 0,69 & 0,19 & 0,06 & 0,16 \\
\hline & & & 6,3Mg@250kPa & 12 & 0,71 & 0,09 & 0,02 & 0,07 \\
\hline & & $30-39$ & 6,3Mg@160kPa & 12 & 1,26 & 0,16 & 0,05 & 0,13 \\
\hline & & & 6,3Mg@250kPa & 12 & 1,28 & 0,15 & 0,04 & 0,12 \\
\hline & NRW kons & $5-14$ & 6,3Mg@160kPa & 12 & 0,71 & 0,05 & 0,01 & 0,04 \\
\hline & & & 6,3Mg@250kPa & 12 & 0,68 & 0,07 & 0,02 & 0,06 \\
\hline & & $18-27$ & 6,3Mg@160kPa & 12 & 0,68 & 0,05 & 0,01 & 0,04 \\
\hline & & & 6,3Mg@250kPa & 12 & 0,83 & 0,07 & 0,02 & 0,06 \\
\hline & & $30-39$ & 6,3Mg@160kPa & 12 & 0,63 & 0,06 & 0,02 & 0,05 \\
\hline & & & 6,3Mg@250kPa & 12 & 0,63 & 0,11 & 0,03 & 0,09 \\
\hline & $\mathrm{NRW}_{\text {konv }}$ & $5-14$ & 6,3Mg@160kPa & 18 & 0,62 & 0,07 & 0,02 & 0,05 \\
\hline & & & 6,3Mg@250kPa & 18 & 0,65 & 0,05 & 0,01 & 0,03 \\
\hline & & $18-27$ & 6,3Mg@160kPa & 18 & 0,59 & 0,03 & 0,01 & 0,02 \\
\hline & & & 6,3Mg@250kPa & 18 & 0,64 & 0,04 & 0,01 & 0,03 \\
\hline & & $30-39$ & 6,3Mg@160kPa & 18 & 0,86 & 0,14 & 0,03 & 0,09 \\
\hline & & & 6,3Mg@250kPa & 18 & 0,82 & 0,11 & 0,03 & 0,07 \\
\hline
\end{tabular}


Tabelle A 44: Effekt des Faktors Reifeninnendruck bei 7,5 Mg Radlast auf die horizontalen Eindringwiderstände [MPa] nach Jahr, Fläche und Messtiefenbereich

\begin{tabular}{|c|c|c|c|c|c|c|c|c|}
\hline Jahr & Fläche & MTB [cm] & Variante & $\mathrm{n}$ & $\mathrm{M}$ & SD & SED & $\mathrm{GD}_{5} \%$ \\
\hline \multirow[t]{24}{*}{2006} & \multirow[t]{6}{*}{$\mathrm{SH}_{\text {kons }}$} & \multirow[t]{2}{*}{$5-14$} & 7,5Mg@250kPa & 12 & 0,36 & 0,04 & 0,01 & 0,03 \\
\hline & & & 7,5Mg@350kPa & 18 & 0,39 & 0,06 & 0,01 & 0,04 \\
\hline & & \multirow[t]{2}{*}{$18-27$} & 7,5Mg@250kPa & 12 & 0,82 & 0,09 & 0,03 & 0,07 \\
\hline & & & 7,5Mg@350kPa & 18 & 0,86 & 0,12 & 0,03 & 0,08 \\
\hline & & \multirow[t]{2}{*}{$30-39$} & 7,5Mg@250kPa & 12 & 1,32 & 0,12 & 0,03 & 0,09 \\
\hline & & & 7,5Mg@350kPa & 18 & 1,35 & 0,12 & 0,03 & 0,08 \\
\hline & \multirow[t]{6}{*}{$\mathrm{SH}_{\text {konv }}$} & \multirow[t]{2}{*}{$5-14$} & 7,5Mg@250kPa & 12 & 0,84 & 0,14 & 0,04 & 0,12 \\
\hline & & & 7,5Mg@350kPa & 18 & 0,96 & 0,22 & 0,05 & 0,15 \\
\hline & & \multirow[t]{2}{*}{$18-27$} & 7,5Mg@250kPa & 12 & 0,90 & 0,17 & 0,05 & 0,14 \\
\hline & & & 7,5Mg@350kPa & 18 & 0,97 & 0,20 & 0,05 & 0,13 \\
\hline & & \multirow[t]{2}{*}{$30-39$} & 7,5Mg@250kPa & 12 & 2,02 & 0,87 & 0,25 & 0,70 \\
\hline & & & 7,5Mg@350kPa & 18 & 1,99 & 0,33 & 0,08 & 0,22 \\
\hline & \multirow[t]{6}{*}{ NRW $W_{\text {kons }}$} & \multirow[t]{2}{*}{$5-14$} & 7,5Mg@250kPa & 12 & 0,75 & 0,06 & 0,02 & 0,05 \\
\hline & & & 7,5Mg@350kPa & 24 & 0,76 & 0,15 & 0,03 & 0,08 \\
\hline & & \multirow[t]{2}{*}{$18-27$} & 7,5Mg@250kPa & 12 & 1,11 & 0,10 & 0,03 & 0,08 \\
\hline & & & 7,5Mg@350kPa & 24 & 1,14 & 0,11 & 0,02 & 0,06 \\
\hline & & \multirow[t]{2}{*}{$30-39$} & 7,5Mg@250kPa & 12 & 0,90 & 0,19 & 0,06 & 0,15 \\
\hline & & & 7,5Mg@350kPa & 24 & 0,95 & 0,14 & 0,03 & 0,08 \\
\hline & \multirow[t]{6}{*}{$\mathrm{NRW}_{\text {konv }}$} & \multirow[t]{2}{*}{$5-14$} & 7,5Mg@250kPa & 18 & 0,60 & 0,06 & 0,01 & 0,04 \\
\hline & & & 7,5Mg@350kPa & 18 & 0,58 & 0,08 & 0,02 & 0,05 \\
\hline & & \multirow[t]{2}{*}{$18-27$} & 7,5Mg@250kPa & 18 & 0,64 & 0,10 & 0,02 & 0,06 \\
\hline & & & 7,5Mg@350kPa & 18 & 0,71 & 0,08 & 0,02 & 0,05 \\
\hline & & \multirow[t]{2}{*}{$30-39$} & 7,5Mg@250kPa & 18 & 1,07 & 0,23 & 0,05 & 0,15 \\
\hline & & & 7,5Mg@350kPa & 18 & 1,05 & 0,29 & 0,07 & 0,19 \\
\hline \multirow[t]{10}{*}{2007} & \multirow[t]{6}{*}{$\mathrm{SH}_{\text {kons }}$} & \multirow[t]{2}{*}{$5-14$} & 7,5Mg@250kPa & 12 & 0,56 & 0,21 & 0,06 & 0,17 \\
\hline & & & 7,5Mg@350kPa & 18 & 0,52 & 0,09 & 0,02 & 0,06 \\
\hline & & \multirow[t]{2}{*}{$18-27$} & 7,5Mg@250kPa & 12 & 1,05 & 0,20 & 0,06 & 0,16 \\
\hline & & & 7,5Mg@350kPa & 18 & 0,95 & 0,12 & 0,03 & 0,08 \\
\hline & & \multirow[t]{2}{*}{$30-39$} & 7,5Mg@250kPa & 12 & 1,45 & 0,26 & 0,08 & 0,21 \\
\hline & & & 7,5Mg@350kPa & 18 & 1,28 & 0,26 & 0,06 & 0,17 \\
\hline & \multirow[t]{4}{*}{$\mathrm{SH}_{\text {konv }}$} & \multirow[t]{2}{*}{$5-14$} & 7,5Mg@250kPa & 12 & 0,78 & 0,22 & 0,06 & 0,18 \\
\hline & & & 7,5Mg@350kPa & 18 & 0,72 & 0,25 & 0,06 & 0,17 \\
\hline & & \multirow[t]{2}{*}{$18-27$} & 7,5Mg@250kPa & 12 & 1,05 & 0,11 & 0,03 & 0,09 \\
\hline & & & 7,5Mg@350kPa & 18 & 1,05 & 0,14 & 0,03 & 0,09 \\
\hline
\end{tabular}




\begin{tabular}{|c|c|c|c|c|c|c|c|c|}
\hline & \multirow[t]{6}{*}{$\mathrm{NRW}_{\text {kons }}$} & \multirow[t]{2}{*}{$5-14$} & 7,5Mg@250kPa & 12 & 0,77 & 0,12 & 0,04 & 0,10 \\
\hline & & & 7,5Mg@350kPa & 24 & 0,78 & 0,10 & 0,02 & 0,06 \\
\hline & & \multirow[t]{2}{*}{$18-27$} & 7,5Mg@250kPa & 12 & 1,08 & 0,07 & 0,02 & 0,06 \\
\hline & & & 7,5Mg@350kPa & 24 & 1,05 & 0,13 & 0,03 & 0,08 \\
\hline & & \multirow[t]{2}{*}{$30-39$} & 7,5Mg@250kPa & 12 & 1,01 & 0,15 & 0,04 & 0,12 \\
\hline & & & 7,5Mg@350kPa & 24 & 1,05 & 0,19 & 0,04 & 0,11 \\
\hline & \multirow[t]{6}{*}{$\mathrm{NRW}_{\text {konv }}$} & \multirow[t]{2}{*}{$5-14$} & 7,5Mg@250kPa & 18 & 0,56 & 0,08 & 0,02 & 0,05 \\
\hline & & & 7,5Mg@350kPa & 18 & 0,61 & 0,11 & 0,03 & 0,07 \\
\hline & & \multirow[t]{2}{*}{$18-27$} & 7,5Mg@250kPa & 18 & 0,71 & 0,06 & 0,01 & 0,04 \\
\hline & & & 7,5Mg@350kPa & 18 & 0,69 & 0,12 & 0,03 & 0,08 \\
\hline & & \multirow[t]{2}{*}{$30-39$} & 7,5Mg@250kPa & 18 & 1,14 & 0,14 & 0,03 & 0,09 \\
\hline & & & 7,5Mg@350kPa & 18 & 1,17 & 0,31 & 0,07 & 0,20 \\
\hline \multirow[t]{24}{*}{2008} & \multirow[t]{6}{*}{$\mathrm{SH}_{\text {kons }}$} & \multirow[t]{2}{*}{$5-14$} & 7,5Mg@250kPa & 12 & 0,59 & 0,05 & 0,02 & 0,04 \\
\hline & & & 7,5Mg@350kPa & 18 & 0,60 & 0,10 & 0,02 & 0,07 \\
\hline & & \multirow[t]{2}{*}{$18-27$} & 7,5Mg@250kPa & 12 & 0,93 & 0,09 & 0,03 & 0,07 \\
\hline & & & 7,5Mg@350kPa & 18 & 0,97 & 0,13 & 0,03 & 0,09 \\
\hline & & \multirow[t]{2}{*}{$30-39$} & 7,5Mg@250kPa & 12 & 1,18 & 0,13 & 0,04 & 0,10 \\
\hline & & & 7,5Mg@350kPa & 18 & 1,13 & 0,16 & 0,04 & 0,10 \\
\hline & \multirow[t]{6}{*}{$\mathrm{SH}_{\text {konv }}$} & \multirow[t]{2}{*}{$5-14$} & 7,5Mg@250kPa & 12 & 0,50 & 0,12 & 0,04 & 0,10 \\
\hline & & & 7,5Mg@350kPa & 18 & 0,53 & 0,16 & 0,04 & 0,10 \\
\hline & & \multirow[t]{2}{*}{$18-27$} & 7,5Mg@250kPa & 12 & 0,65 & 0,07 & 0,02 & 0,06 \\
\hline & & & 7,5Mg@350kPa & 18 & 0,78 & 0,13 & 0,03 & 0,09 \\
\hline & & \multirow[t]{2}{*}{$30-39$} & 7,5Mg@250kPa & 12 & 1,28 & 0,25 & 0,07 & 0,20 \\
\hline & & & 7,5Mg@350kPa & 18 & 1,49 & 0,57 & 0,13 & 0,37 \\
\hline & \multirow[t]{6}{*}{$\mathrm{NRW}_{\text {kons }}$} & \multirow[t]{2}{*}{$5-14$} & 7,5Mg@250kPa & 12 & 0,77 & 0,06 & 0,02 & 0,05 \\
\hline & & & 7,5Mg@350kPa & 24 & 0,71 & 0,08 & 0,02 & 0,04 \\
\hline & & \multirow[t]{2}{*}{$18-27$} & 7,5Mg@250kPa & 12 & 0,77 & 0,06 & 0,02 & 0,05 \\
\hline & & & 7,5Mg@350kPa & 24 & 0,81 & 0,10 & 0,02 & 0,06 \\
\hline & & \multirow[t]{2}{*}{$30-39$} & 7,5Mg@250kPa & 12 & 0,67 & 0,04 & 0,01 & 0,03 \\
\hline & & & 7,5Mg@350kPa & 24 & 0,62 & 0,13 & 0,03 & 0,08 \\
\hline & \multirow[t]{6}{*}{$\mathrm{NRW}_{\text {konv }}$} & \multirow[t]{2}{*}{$5-14$} & 7,5Mg@250kPa & 18 & 0,69 & 0,07 & 0,02 & 0,04 \\
\hline & & & 7,5Mg@350kPa & 18 & 0,69 & 0,10 & 0,02 & 0,06 \\
\hline & & \multirow[t]{2}{*}{$18-27$} & 7,5Mg@250kPa & 18 & 0,65 & 0,06 & 0,01 & 0,04 \\
\hline & & & 7,5Mg@350kPa & 18 & 0,64 & 0,03 & 0,01 & 0,02 \\
\hline & & \multirow[t]{2}{*}{$30-39$} & 7,5Mg@250kPa & 18 & 0,87 & 0,25 & 0,06 & 0,17 \\
\hline & & & 7,5Mg@350kPa & 18 & 0,81 & 0,10 & 0,02 & 0,06 \\
\hline
\end{tabular}


Tabelle A 45: Horizontaler Eindringwiderstand [MPa] nach Jahr und Fläche in Abhängigkeit der Anzahl der zuvor erfolgten TASIS-Einsätze an selben Stelle

\begin{tabular}{|c|c|c|c|c|c|c|c|}
\hline Jahr & Fläche & $\begin{array}{l}\text { Anzahl der TASIS- } \\
\text { Einsätze }\end{array}$ & $\mathrm{n}$ & M & SD & SED & $\mathrm{GD}_{5 \%}$ \\
\hline \multirow[t]{8}{*}{2007} & $\mathrm{SH}_{\text {kons }}$ & 1. TASIS-Einsatz & 288 & 0,97 & 0,37 & 0,02 & 0,06 \\
\hline & & 2. TASIS-Einsatz & 279 & 0,97 & 0,38 & 0,02 & 0,06 \\
\hline & $\mathrm{SH}_{\mathrm{konv}}$ & 1. TASIS-Einsatz & 192 & 0,90 & 0,22 & 0,02 & 0,04 \\
\hline & & 2. TASIS-Einsatz & 188 & 0,86 & 0,30 & 0,02 & 0,06 \\
\hline & $N R W_{\text {kons }}$ & 1. TASIS-Einsatz & 324 & 0,95 & 0,18 & 0,01 & 0,03 \\
\hline & & 2. TASIS-Einsatz & 324 & 0,93 & 0,20 & 0,01 & 0,03 \\
\hline & $\mathrm{NRW}_{\text {konv }}$ & 1. TASIS-Einsatz & 432 & 0,78 & 0,25 & 0,01 & 0,03 \\
\hline & & 2. TASIS-Einsatz & 432 & 0,83 & 0,35 & 0,02 & 0,05 \\
\hline \multirow[t]{12}{*}{2008} & $\mathrm{SH}_{\text {kons }}$ & 1. TASIS-Einsatz & 261 & 0,85 & 0,28 & 0,02 & 0,05 \\
\hline & & 2. TASIS-Einsatz & 267 & 0,64 & 0,20 & 0,01 & 0,03 \\
\hline & & 3. TASIS-Einsatz & 288 & 0,69 & 0,23 & 0,01 & 0,04 \\
\hline & $\mathrm{SH}_{\mathrm{konv}}$ & 1. TASIS-Einsatz & 384 & 0,96 & 0,43 & 0,02 & 0,06 \\
\hline & & 2. TASIS-Einsatz & 288 & 0,77 & 0,37 & 0,02 & 0,06 \\
\hline & & 3. TASIS-Einsatz & 192 & 0,56 & 0,16 & 0,01 & 0,03 \\
\hline & $N R W_{\text {kons }}$ & 1. TASIS-Einsatz & 324 & 0,71 & 0,10 & 0,01 & 0,02 \\
\hline & & 2. TASIS-Einsatz & 324 & 0,67 & 0,12 & 0,01 & 0,02 \\
\hline & & 3. TASIS-Einsatz & 324 & 0,64 & 0,12 & 0,01 & 0,02 \\
\hline & NRW $W_{\text {konv }}$ & 1. TASIS-Einsatz & 432 & 0,71 & 0,17 & 0,01 & 0,02 \\
\hline & & 2. TASIS-Einsatz & 432 & 0,71 & 0,17 & 0,01 & 0,02 \\
\hline & & 3. TASIS-Einsatz & 432 & 0,72 & 0,19 & 0,01 & 0,03 \\
\hline
\end{tabular}


Tabelle A 46: Horizontaler Eindringwiderstand [MPa] nach Bodenbearbeitung (BB) und Messtiefenbereich in Abhängigkeit der Anzahl der zuvor erfolgten TASIS-Einsätze an selber Stelle

\begin{tabular}{llllllll}
\hline BB & MTB [cm] & $\begin{array}{l}\text { Anzahl der TASIS- } \\
\text { Einsätze }\end{array}$ & $n$ & $M$ & SD & SED & GD $_{5 \%}$ \\
\hline kons. & $5-14$ & 1. TASIS-Einsatz & 195 & 0,64 & 0,13 & 0,01 & 0,03 \\
& & 2. TASIS-Einsatz & 197 & 0,62 & 0,13 & 0,01 & 0,03 \\
& \multirow{3}{*}{$18-27$} & 3. TASIS-Einsatz & 204 & 0,63 & 0,14 & 0,01 & 0,03 \\
& 1. TASIS-Einsatz & 195 & 0,81 & 0,12 & 0,01 & 0,02 \\
& 2. TASIS-Einsatz & 197 & 0,71 & 0,16 & 0,01 & 0,03 \\
& 3. TASIS-Einsatz & 204 & 0,71 & 0,18 & 0,01 & 0,04 \\
& \multirow{3}{*}{$30-39$} & 1. TASIS-Einsatz & 195 & 0,86 & 0,28 & 0,02 & 0,06 \\
& & 2. TASIS-Einsatz & 197 & 0,63 & 0,18 & 0,01 & 0,04 \\
& \multirow{4}{*}{$5-14$} & 3. TASIS-Einsatz & 204 & 0,64 & 0,20 & 0,01 & 0,04 \\
& 1. TASIS-Einsatz & 240 & 0,57 & 0,12 & 0,01 & 0,02 \\
& 2. TASIS-Einsatz & 240 & 0,58 & 0,13 & 0,01 & 0,02 \\
& 3. TASIS-Einsatz & 240 & 0,57 & 0,14 & 0,01 & 0,03 \\
& \multirow{2}{*}{$18-27$} & 1. TASIS-Einsatz & 240 & 0,64 & 0,10 & 0,01 & 0,02 \\
& 2. TASIS-Einsatz & 240 & 0,62 & 0,10 & 0,01 & 0,02 \\
& 3. TASIS-Einsatz & 240 & 0,64 & 0,10 & 0,01 & 0,02 \\
& 1. TASIS-Einsatz & 336 & 1,14 & 0,31 & 0,02 & 0,05 \\
& \multirow{2}{*}{$30-39$} & 2. TASIS-Einsatz & 240 & 1,01 & 0,28 & 0,02 & 0,05 \\
& 3. TASIS-Einsatz & 144 & 0,90 & 0,20 & 0,02 & 0,05 \\
\hline
\end{tabular}


Tabelle A 47: Ruhedruckkoeffizient nach Fläche, Messtiefenbereich und Radlast

\begin{tabular}{|c|c|c|c|c|c|c|c|}
\hline Fläche & MTB [cm] & $\mathrm{RL}[\mathrm{Mg}]$ & $\mathrm{n}$ & M & SD & SED & $\mathrm{GD}_{5 \%}$ \\
\hline \multirow[t]{12}{*}{$\mathrm{SH}_{\text {kons }}$} & \multirow[t]{4}{*}{$5-14$} & unbefahren & 42 & 0,49 & 0,16 & 0,02 & 0,07 \\
\hline & & 3,3 & 72 & 0,69 & 0,23 & 0,03 & 0,08 \\
\hline & & 6,3 & 70 & 0,66 & 0,23 & 0,03 & 0,08 \\
\hline & & 7,5 & 90 & 0,62 & 0,21 & 0,02 & 0,06 \\
\hline & \multirow[t]{4}{*}{$18-27$} & unbefahren & 42 & 0,44 & 0,17 & 0,03 & 0,07 \\
\hline & & 3,3 & 72 & 0,51 & 0,11 & 0,01 & 0,04 \\
\hline & & 6,3 & 70 & 0,53 & 0,13 & 0,02 & 0,04 \\
\hline & & 7,5 & 90 & 0,52 & 0,12 & 0,01 & 0,04 \\
\hline & \multirow[t]{4}{*}{$30-39$} & unbefahren & 42 & 0,65 & 0,61 & 0,09 & 0,26 \\
\hline & & 3,3 & 72 & 0,63 & 0,25 & 0,03 & 0,08 \\
\hline & & 6,3 & 70 & 0,73 & 0,32 & 0,04 & 0,11 \\
\hline & & 7,5 & 90 & 0,67 & 0,20 & 0,02 & 0,06 \\
\hline \multirow[t]{12}{*}{$\mathrm{SH}_{\text {konv }}$} & \multirow[t]{4}{*}{$5-14$} & unbefahren & 51 & 0,40 & 0,19 & 0,03 & 0,08 \\
\hline & & 3,3 & 68 & 0,44 & 0,16 & 0,02 & 0,05 \\
\hline & & 6,3 & 68 & 0,38 & 0,12 & 0,01 & 0,04 \\
\hline & & 7,5 & 85 & 0,40 & 0,15 & 0,02 & 0,04 \\
\hline & \multirow[t]{4}{*}{$18-27$} & unbefahren & 51 & 0,44 & 0,18 & 0,03 & 0,07 \\
\hline & & 3,3 & 67 & 0,42 & 0,13 & 0,02 & 0,04 \\
\hline & & 6,3 & 68 & 0,44 & 0,14 & 0,02 & 0,05 \\
\hline & & 7,5 & 85 & 0,44 & 0,18 & 0,02 & 0,05 \\
\hline & \multirow[t]{4}{*}{$30-39$} & unbefahren & 33 & 0,46 & 0,14 & 0,02 & 0,07 \\
\hline & & 3,3 & 44 & 0,54 & 0,24 & 0,04 & 0,10 \\
\hline & & 6,3 & 44 & 0,44 & 0,12 & 0,02 & 0,05 \\
\hline & & 7,5 & 55 & 0,51 & 0,22 & 0,03 & 0,08 \\
\hline \multirow[t]{12}{*}{$\mathrm{NRW}_{\text {kons }}$} & \multirow[t]{4}{*}{$5-14$} & unbefahren & 60 & 0,49 & 0,14 & 0,02 & 0,05 \\
\hline & & 3,3 & 72 & 0,59 & 0,18 & 0,02 & 0,06 \\
\hline & & 6,3 & 72 & 0,57 & 0,17 & 0,02 & 0,06 \\
\hline & & 7,5 & 96 & 0,59 & 0,18 & 0,02 & 0,05 \\
\hline & \multirow[t]{4}{*}{$18-27$} & unbefahren & 60 & 0,47 & 0,13 & 0,02 & 0,05 \\
\hline & & 3,3 & 72 & 0,45 & 0,14 & 0,02 & 0,05 \\
\hline & & 6,3 & 72 & 0,45 & 0,09 & 0,01 & 0,03 \\
\hline & & 7,5 & 96 & 0,50 & 0,13 & 0,01 & 0,04 \\
\hline & \multirow[t]{4}{*}{$30-39$} & unbefahren & 60 & 0,53 & 0,16 & 0,02 & 0,06 \\
\hline & & 3,3 & 72 & 0,48 & 0,23 & 0,03 & 0,08 \\
\hline & & 6,3 & 72 & 0,48 & 0,14 & 0,02 & 0,05 \\
\hline & & 7,5 & 96 & 0,54 & 0,22 & 0,02 & 0,06 \\
\hline
\end{tabular}




\begin{tabular}{lllllll} 
NRW konv $^{2}-14$ & unbefahren & 54 & 0,46 & 0,12 & 0,02 & 0,05 \\
& nur Schlepper & 54 & 0,48 & 0,10 & 0,01 & 0,04 \\
& 3,3 & 108 & 0,49 & 0,12 & 0,01 & 0,03 \\
& 6,3 & 108 & 0,49 & 0,13 & 0,01 & 0,03 \\
& 7,5 & 108 & 0,53 & 0,15 & 0,01 & 0,04 \\
& unbefahren & 54 & 0,52 & 0,16 & 0,02 & 0,06 \\
& nur Schlepper & 54 & 0,52 & 0,17 & 0,02 & 0,07 \\
& 3,3 & 108 & 0,50 & 0,12 & 0,01 & 0,03 \\
& 6,3 & 108 & 0,46 & 0,10 & 0,01 & 0,03 \\
& $7,5-39$ & 108 & 0,48 & 0,11 & 0,01 & 0,03 \\
& unbefahren & 54 & 0,41 & 0,17 & 0,02 & 0,07 \\
& nur Schlepper & 54 & 0,46 & 0,23 & 0,03 & 0,09 \\
& 3,3 & 108 & 0,49 & 0,18 & 0,02 & 0,05 \\
& 6,3 & 108 & 0,43 & 0,14 & 0,01 & 0,04 \\
& 7,5 & 108 & 0,46 & 0,15 & 0,01 & 0,04 \\
\hline
\end{tabular}

Tabelle A 48: Ruhedruckkoeffizient nach Bodenbearbeitung (BB) und Messtiefenbereich (MTB)

\begin{tabular}{lllllll}
\hline BB & MTB $[\mathrm{cm}]$ & $\mathrm{n}$ & $\mathrm{M}$ & $\mathrm{SD}$ & $\mathrm{SED}$ & $\mathrm{GD}_{5 \%}$ \\
\hline kons. & $5-14$ & 574 & 0,59 & 0,20 & 0,01 & 0,02 \\
& $18-27$ & 574 & 0,49 & 0,13 & 0,01 & 0,02 \\
& $30-39$ & 574 & 0,59 & 0,28 & 0,01 & 0,03 \\
konv. & $5-14$ & 704 & 0,46 & 0,15 & 0,01 & 0,02 \\
& $18-27$ & 703 & 0,47 & 0,14 & 0,01 & 0,02 \\
& $30-39$ & 608 & 0,46 & 0,18 & 0,01 & 0,02 \\
\hline
\end{tabular}


Tabelle A 49: Ergebnisse der erweiterten Spatendiagnose vom 06.10.2008 auf der Fläche NRW (nach DBG 2005)

\begin{tabular}{lllllll}
\hline Merkmal & \multicolumn{2}{c}{$\underline{\mathrm{K} 0}$} & \multicolumn{2}{c}{$\underline{\mathrm{B} 3}$} & \multicolumn{2}{c}{$\underline{\text { GEO }}$} \\
& Tiefe $[\mathrm{cm}]$ & Bewertung & Tiefe $[\mathrm{cm}]$ & Bewertung & Tiefe $[\mathrm{cm}]$ & Bewertung \\
\hline Beschaffenheit der & $0-20$ & 4 & $0-10$ & 3 & $0-20$ & 4 \\
Bodenoberfläche & $0-20$ & 1 & $0-10$ & 1 & $0-20$ & 1 \\
Gefügeform und & $20-35$ & 2 & $10-35$ & 3 & $20-35$ & 2 \\
Bewertung & $35-50$ & 4 & $35-50$ & 4 & $35-50$ & 4 \\
& $0-20$ & 1 & $0-10$ & 1 & $0-20$ & 1 \\
Wurzeln & $20-35$ & 1 & $10-35$ & 2 & $20-35$ & 1 \\
(Durchwurzelung) & $35-50$ & 4 & $35-50$ & 4 & $35-50$ & 4 \\
& $0-20$ & 1 & $0-10$ & 1 & $0-20$ & 1 \\
Farbe, Geruch & $20-35$ & 2 & $10-35$ & 2 & $20-35$ & 2 \\
(Durchlüftung) & $35-50$ & 4 & $35-50$ & 3 & $35-50$ & 4 \\
& $0-20$ & 1 & $0-10$ & 1 & $0-20$ & 1 \\
Ernterückstände & $20-35$ & 5 & $10-35$ & 5 & $20-35$ & 5 \\
& $35-50$ & 1 & $35-50$ & 1 & $35-50$ & 1 \\
Röhren, Klüfte & $0-20$ & 1 & $0-10$ & 1 & $0-20$ & 1 \\
& $20-35$ & 3 & $10-35$ & 3 & $35-50$ & 3 \\
& $35-50$ & 4 & $35-50$ & 4 & $35-50$ & 4 \\
Übergang zwischen & $0-20$ & 5 & $0-10$ & 5 & $0-20$ & 5 \\
Schichten und & $20-35$ & 5 & $10-35$ & 5 & $20-35$ & 5 \\
Horizonten & $35-50$ & 5 & $35-50$ & 5 & $35-50$ & 5 \\
\hline & & & & & &
\end{tabular}

